\title{
Jörg Kaltwasser
}

\section{Die deadjektivische Wortbildung \\ des Russischen}

\section{Versuch einer "analytisch-synthetisch- funktionellen" Beschreibung}

Verlag Otto Sagner München · Berlin · Washington D.C.

Digitalisiert im Rahmen der Kooperation mit dem DFG-Projekt „Digi20“

der Bayerischen Staatsbibliothek, München. OCR-Bearbeitung und Erstellung des eBooks durch den Verlag Otto Sagner:

http://verlag.kubon-sagner.de

() bei Verlag Otto Sagner. Eine Verwertung oder Weitergabe der Texte und Abbildungen, insbesondere durch Vervielfältigung, ist ohne vorherige schriftliche Genehmigung des Verlages unzulässig. 


\title{
SLAVISTISCHE BEITRÄGE
}

\author{
BEGRÜNDET VON \\ ALOIS SCHMAUS \\ HERAUSGEGEBEN VON \\ JOHANNES HOLTHUSEN $† \cdot$ HEINRICH KUNSTMANN \\ PETER REHDER · JOSEF SCHRENK \\ REDAKTION \\ PETER REHDER \\ Band 190
}




\section{JÖRG KALTWASSER \\ DIE DEADJEKTIVISCHE WORTBILDUNG \\ DES RUSSISCHEN \\ Versuch einer \\ ,analytisch-synthetisch-funktionellen' \\ Beschreibung}

\section{VERLAG OTTO SAGNER · MÜNCHEN 1986}


ISBN 3.87690-325.4

(C) Verlag Otto Sagner, München 1986

Abteilung der Firma Kubon \& Sagner, München

Druck: D. Gräbner, Altendorf

\section{Bayerlscho}

Staatsbibllothon

Münchon 
VORWORT

Der Plan, die vorliegende Arbeit zu verfassen, reifte bereits Ende der siebziger Jahre, als an der Universität Konstanz unter der federführung von Prof. W. Lehfeldt die sogenannte 'analytisch-synthetisch-funktionelle' Untersuchung der Formenbildung entwickelt und an slavischen Sprachen erprobt wurde. Es lag nahe, diese Methode hinsichtlich ihrer Eignung für andere Gebiete sprachwissenschaftlicher Forschung zu überprüfen. Die Wortbildung kam deshalb in Betracht, weil man sich mit ihr in der Sowjetunion besonders intensiv auseinandersetzt(e), ohne daB bisher eine kohärente Metasprache, geschweige denn ein in sich geschlossenes und dabei überprüfbares Beschreibungsverfahren entwickelt worden wäre.

Die Sichtung der grundlegenden Literatur konnte ich im Rahmen eines vom DAAD geförderten zehnmonatigen Aufenthaltes in Moskau 1980/81 leisten. Dort betreute mich Prof. I.S. Uluchanov, dem ich für seine allzeit bereitwillige Unterstützung danken möchte. Dank schulde ich in diesem Zusammenhang auch meiner Frau, die diesen Aufenthalt gebilligt und die damit verbundene Trennung in Kauf genommen hat.

Die Arbeit ist, was den empirischen Teil anbelangt, in erster Linie für Slavisten bestimmt, bietet in den übrigen Kapiteln aber auch dem on Problemen der Wortbildung interessierten Nicht-Slavisten einen Überblick insbesondere über den aktuellen Stand der sowjetischen forschung. Bedeutung über die Einzelsprache hinaus hat der erste, der begriffskritische Teil der Arbeit. Damit die dort erzielten Ergebnisse jedermann zugänglich sind, habe ich alle russischen $\mathrm{Zitate,} \mathrm{mit} \mathrm{Ausnahme} \mathrm{der} \mathrm{in}$ den Anmerkungen befindlichen, übersetzt.

Die Publikation verzögerte sich aus Gründen, die mit meinem beruflichen Werdegang zusammenhängen und wäre vielleicht nie gelungen, hätte Prof. Lehfeldt, der die Entwicklung der Arbeit kritisch verfolgte, mich nicht zur Weiterarbeit ermuntert. Deshalb gilt inm mein ganz besonderer Dank.

Werl, in Januar 1986

J.K. 


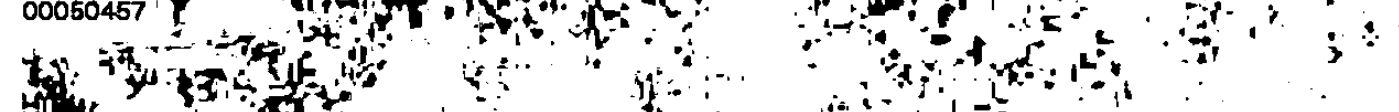

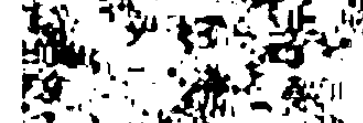

inting

$$
\text { of }
$$

340 in

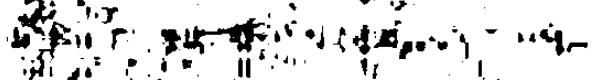

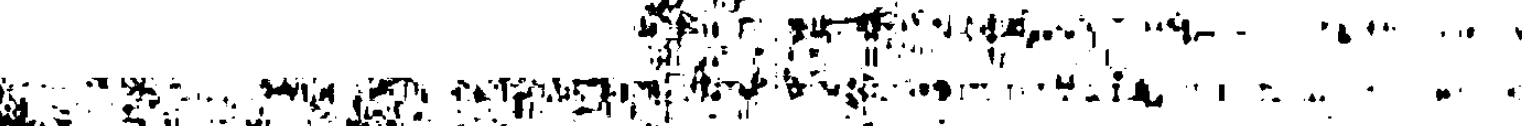

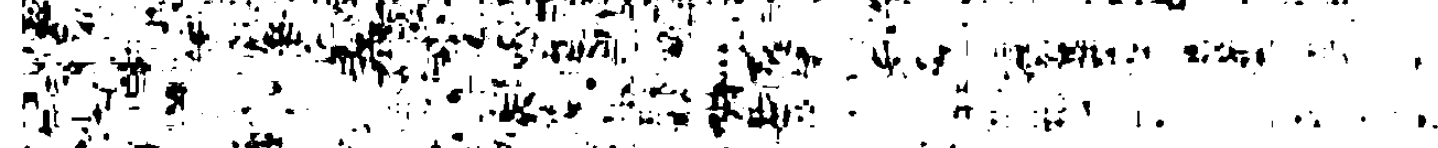

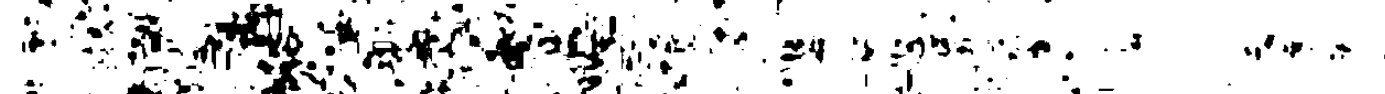

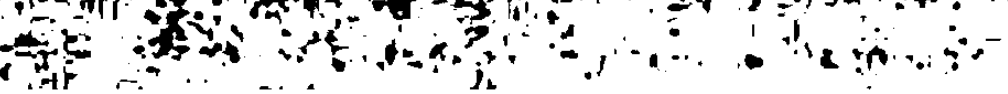

How

Yor

prom

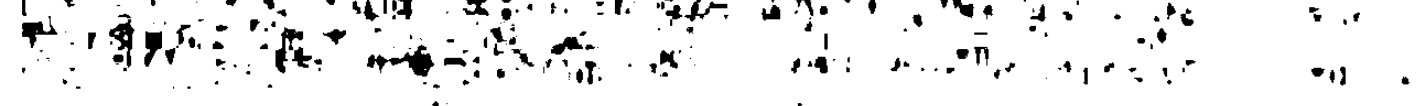

A) int

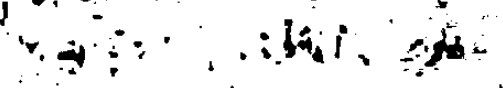

$\because 40$

5. $\because 1 \quad \cdots$

\section{$\therefore$ 种}

4 .

non

Fition

a 10

$4+4+4 y^{2}$

$y_{1}^{2}$

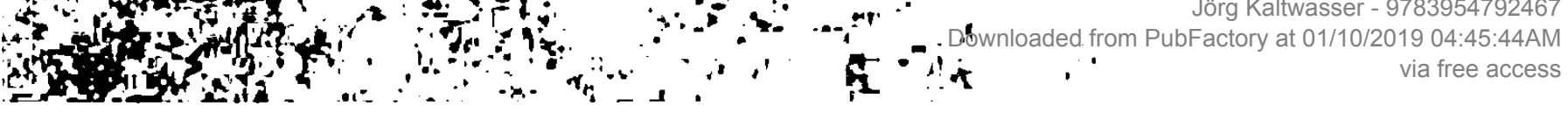


INHAL TSVERZE ICHNIS

0. EINFUHRUNG IN DIE AUFGABENSTELLUNG

1. THEORETISCH-BEGRIFFSKRITISCHER TEIL 7

1.1. Die Stellung der Wortbildung 7

1.1.1. Der Gegenstand der Wortbildung 8

1.2. Sukzessive Einführung in die der empirischen Untersuchung zugrunde liegenden Begrifflichkeit 12

1.2.1. Nichtdefinierte Begriffe 13

1.2.2. Definierte Begriffe

1.2.2.1. Konkrete Wortform 14

1.2.2.2. Abstrakte Wortform 16

1.2.2.3. Grammatische Bedeutung, nichtgrammatische
Bedeutung, Grammem, grammatische Kategorie 16

1.2.2.4. Lexem 18

1.2.2.5. Wortarten 19

1.2.2.6. Flexionsendung, Wortformstamm 27

1.2.2.7. Wurzel der Wortform; Affix 32

1.2.2.8. Derivationsbeziehung 36

1.2.2.9. Suppletivismus 45

1.2.2.10. Derivationsrichtung 48

1.2.2.11. 'Wortbildungsbedeutung' 60

1.2.2.12. Morphonologische Alternation 69

2. EMPIRISCHE ANALYSE DER DEADJEKTIVISCHEN WORTBILDUNG DES RUSSISCHEN

2.1. Zerlegung der (offenen Menge der) Adjektive des Russischen aufgrund der allgemeinen Explikation ihrer nichtgrammatischen Inhaltselemente

2.2. Die nichtgrammatischen Inhaltselemente der deadjektivischen Redeteile in allgemeiner Explikation

2.3. Analytischer Teil

2.3.1. Auflistung der an der deadjektivischen wortbildung des Russischen beteiligten Affixe 
2.3.1.1. Präfixe 88

2.3.1.2. Suffixe 89

2.3.1.3. Kombinationen von Präfix und Suffix 90

2.3.1.4. Kombinationen von Suffix und Postfix 91

2.3.2. Die morphonologischen Veränderungen der Bezugs form

2.3.2.1. Verkürzungen der Bezugsform 91

2.3.2.2. Erweiterungen der Bezugsform 92

2.3.2.3. Vokalalternationen 93

2.3.2.4. Konsonantenalternationen

2.3.2.4.1. Palatalitäts- und Dispalatalitätsalternationen 93

2.3.2.4.2. Transitivitätsalternationen 94

2.3.3. Die Akzentverhältnisse bei der deadjektivischen Derivation des Russischen 95

2.4. Synthetischer Teil

2.4.1. Deadjektivische Substantive mit der Bedeutung eines abstrakten Merkmals

2.4.2. Deadjektivische Substantive mit der Bedeutung des Merkmalsträgers

2.4.3. Deadjektivische Adjektive

2.4.4. Deadjektivische Verben

2.4.5. Deadjektivische Adverbien

2.4.6. Die Bestimmung des 'MaBes der Verbundenheit' für die deadjektivische Derivation des Russischen

2.4.7. Die implikativen Regelhaftigkeiten bei der Kombination der Ausdrucksmittel

2.4.8. Analyse der Ausdrucksmittel hinsichtlich ihrer prädiktiven und ihrer diskriminatorischen Stärke

2.4.9. Darstellung der Korrelationen zwischen den Derivatemtypen und den Graphen

3. ZUSAMMENFASSUNG UND BEWERTUNG DER LEISTUNG DER METHODE 
Zahlreiche Aufsätze, Dissertationen, Monographien und Sammelbände sind bisher zu den verschiedensten Problemen der russischen Wortbildung verfaBt worden ${ }^{1}$; zudem ist der genannte Bereich Gegenstand von Lehrbüchern ${ }^{2}$, Wörterbüchern ${ }^{3}$ und Grammatiken $^{4}$. Inre vorläufig letzte umfassende Bearbeitung hat die Wortbildung des Russischen in der jüngsten Akademiegrammatik $(A G-80)^{5}$ erfahren, die, zumindest was die Kompilation der sprachlichen Daten anbelangt, schwerlich zu übertreffen sein dürfte.

Somit kann es kaum das Ziel der vorliegenden Arbeit sein, neves Material zu präsentieren; auch soll es nicht darum gehen, die Entwicklungstendenzen aufzuzeigen, die die Beschäftigung mit der Wortbildung des Russischen etwa in den letzten 150 Jahren genommen hat ${ }^{6}$, obwohl dies sicherlich eine lohnende Aufgabe wäre, zumal dann, wenn die Untersuchung kontrastiv, z.B. unter gleichzeitiger Berücksichtigung der interessantesten westlichen Ansätze in dem jeweils entsprechenden Zeitraum abgewickelt würde. 7

1 Die Bibliographie von WORTH (1977) enthält lediglich einen (wenn auch nicht unbedeutenden) Ausschnitt aus dem gesamten Spektrum.

2 Vgl. z.B. NOVICKAJA (1968) oder POTICHA (1970).

3 Vgl. bspw. POTICHA (1961), VOLKONSKY, POL TORACKY (1961) oder WORTH et al. (1970).

4 Vgl. z.B. die AG-53/54 (in der die Wortbildung noch als Teil der Morphologie abgehandelt wird) oder die $A G-70$.

5 Als Verfasser des entsprechenden Kapitels zeichnen V.V. LOPAIIN sowie I.S. ULUCHANOV.

6 Eine ungefähre Vorstellung von dem, was über den genannten Zeitraum hinweg (mit Ausnahme der Jahre 1881-1917) in der östlichen Hemisphäre auf dem Gebiet der Wortbildung geleistet wurde, kann man sich U.a. anhand der folgenden Literatur verschaffen: ASNIN (1965), AVILOVA et al. (1954), DEBEC et al. (1958; 1969; 1973), FILIN (1970; 1978), MOŻAEVA (1963a; 1963b). Vor allem das 20. Jahrhundert (bis etwa 1973) behandelt auch ARMSTRONG (1979) mit einem relativ knappen, doch vielschichtigen und daher informativen Überblick über die wichtigsten Forschungsrichtungen auf dem Gebiet der Wortbildung des Russischen, wobei er insbesondere auf Arbeiten von FORTUNATOV, BOGORODICKIJ, VINOKUR, PEŚKOVSKIJ, VINOGRADOV, LOPATIN, ULUCHANOV, SOBOLEVA, ZEMSKAJA und MEL'ĆUK eingeht.

7 Man denke nur an Arbeiten jüngeren Datums von Sprachwissenschaftlern wie 
Im folgenden wird es darum gehen,

a) einige der Mängel aufzuzeigen, mit denen viele der bisher vorliegenden Abhandlungen zur Wortbildung des Russischen behaftet sind, und

b) diesem Konglomerat unterschiedlicher Auffassungen und in sich häufig unzusammenhängender Vorgehensweisen ein kohärentes Verfahren entgegenzusetzen, das sich bereits im Rahmen der Deskription der formenbildung der slavischen Sprachen als geeignet herausgestellt hat.

Wer sich darüber informieren möchte, wie diese sogenannte 'analytisch-synthetisch-funktionelle' Vorgehensweise auf dem zuletzt genannten Gebiet 'funktioniert', sei ouf die Arbeiten VON BERGER (1981), KAL TWASSER, KEMPGEN (1981); KEMPGEN (1979), KEMPGEN, LEHFELDT (1978) sowie LEHFELDT (1977; 1978a; 1978b; 1979; 1980) verwiesen. Die genannte Methode soll auch innerhalb des komplexen Bereichs der russischen Wortbildung den heuristischen Rahmen für die empirische Untersuchung abgeben.

An dieser Stelle sei jedoch bereits hervorgehoben, daB eine direkte Ubertragbarkeit der in den oben angeführten Arbeiten entwickelten Vorgehensweise auf die Wortbildung nicht gegeben ist, da vor allem im Hinblick auf die Inhaltsseiten der jeweils zu betrachtenden Wörter (Wortformen) signifikante Unterschiede bestehen. Während man sich bei der Formenbildung auf die Veränderungen der grammatischen Bedeutungen der Wortformen eines und desselben Lexems (bzw. von Lexemen eines und desselben Redeteils) sowie auf die Mittel zu deren Ausdruck konzentriert, geht es in der vorliegenden Arbeit um solche nichtgrammatischen Inhaltselemente von Wörtern, die unter bestimmten Bedingungen in anderen Wörtern (auch anderer Redetei1e) als sich wiederholend gedacht werden können. Diese letztgenannten Bedeutungen sind nicht nur schwieriger zu 'fassen'

(7) ERBEN (1964; 1975), FLEISCHER ( $\left.{ }^{3} 1974\right)$, GAUGER (1971), HENZEN ( $\left.{ }^{3} 1965\right)$, KÜRSCHNER (1973), MARCHAND $\left({ }^{2} 1969 ; 1974\right)$, MOISCH $(1960 ; 1965)$, V. POLENZ (1968; 1973), WEISGERBER (1964). Diese kleine Liste lieBe sich natürlich durch die Einbeziehung von früher erschienenen Arbeiten z.T. dieser wie auch anderer Autoren sowie durch Berücksichtigung der wichtigsten Linguisten aus dem angelsächsischen Raum nahezu beliebig erweitern. 
und zu schematisieren als die in der formenbildung zu behandelnden Kombinationen grammatischer Bedeutungen; darüber hinaus gestaltet sich auch das 'Zusammenspiel' der zum Ausdruck dieser Bedeutungen dienenden Mittel wesentlich komplexer als dies bei der formenbildung der Fall ist. Aus diesen Gründen (völlig anderer Untersuchungsgegenstand mit daraus resultierenden anderen Untersuchungsmethoden und natürlich auch -ergebnissen) handelt es sich hier nicht um eine Kopie des genannten Verfahrens, sondern um eine eigenständige Konzeption, die den äuBeren Rahmen der 'analytisch-synthetisch-funktionellen' Vorgehensweise mit ganz anderen Inhalten füllt.

Von der zu leistenden Beschreibung der deadjektivischen Derivation wird gefordert, daB sie von nachprüfbaren sprachlichen Beobachtungsdaten auszugehen und somit den Fakten der sprachlichen Wirklichkeit Rechnung zu tragen hat. 8 AuBerdem soll sie einen Einblick in die Zusammenhänge der beschriebenen Sprachdaten gestatten, indem sie nicht allein auf die wiederholbarkeit dieses oder jenes Sachverhalts hinweist, sondern statt dessen bestimmte Gesetzmäßigkeiten oder Regularitäten bezüglich der Auftretensbedingungen der beschriebenen Erscheinung aufzudecken sucht. 9

Zielsetzung und Konzeption der vorliegenden Untersuchung lagazn sich kurz folgendermaBen umreiben: Es wird darum gehen, zu beschreiben, in welcher Weise die nichtgrammatischen Bedeutungen von Lexemen, die im Russischen dem Redeteil 'Adjektiv' zuzuordnen sind, in anderen Lexemen desselben Redeteils oder anderer Redeteile modifiziert werden. - Die deadjektivische Derivation wurde aus rein pragmatischen Gründen zum Untersuchungagegenstand bestimmt. So war darauf zu achten, die empirische Basis für die beabsichtigte Analyse einerseits nicht zu schmal (was beispielsweise auf die denumerale Wortbildung zuträfe), andererseits jedoch auch nicht zu umfangreich für den dieser Arbeit gesteckten Rahmen (wie dies z.B. bei der desubstantivischen Derivation des Russischen der Fall wäre) zu wäh-

8 Zum Begriff 'sprachliche Wirklichkeit' vgl. GRUCZA (1970, 40ff).

9 Vgl. hierzu vor allem STEGMULLER (1969). 
len.

Im Unterschied zu den meisten anderen Arbeiten, die einen Redeteil herausgreifen und dann analysieren, von welchen Redeteilen die inm angehörigen Lexeme deriviert sind, wird hier eine andere Richtung verfolgt. Es soll gezeigt werden,

a) welche Wörter aus welchen Redeteilen von Angehörigen eines bestimmten Redeteils (des Redeteils 'Adjektiv') als abgeleitet gedacht werden können und

b) über welche Ausdrucksmittel das Russische verfügt, um aus diesem fundus von Lexemen mit bestimmten Bedeutungen und formen andere Lexeme mit modifizierten Bedeutungen abzuleiten, ferner,

c) wie diese Mittel kombiniert werden und schlieblich

d) wie sie sich im Hinblick auf ihre funktionelle Belastung (functional load, funkcional'naja nagruzka) voneinander unterscheiden.

Die Arbeit umfaBt drei Teile:

1. Einen theoretisch-begriffskritischen.

2. Einen empiriachen, der wiederum gegliedert ist in

2.1. einen klassifikatorischen,

2.2. einen analytigchen,

2.3. einen synthetischen sowie in

2.4. einen (im obigen Sinne) funktionellen.

3. Eine kritische Würdigung der gewählten Heuristik.

Ad (1) Der theoretisch-begriffskritische Teil

Aus der überlegung heraus, daB die Wortbildung alle Wörter einer gegebenen Sprache unter dem Aspekt inres inneren Aufbaus zu untersuchen hat, ist der Weg anzugeben, der zu der Objektmenge führt, die es zu analysieren gilt. Dabei sollen sämtliche benötigten Begriffe von einer explizit zu nennenden Grundeinheit in nachprüfbarer und nachvollziehbarer Weise möglichst auf der Basis rein linguistischer Operationen sukzessiv abgeleitet und definiert werden. Der erste Teil der Arbeit soll also einen nach Möglichkeit kohärenten Begriffsapparat für die nachfolgende empirische Untersuchung liefern und somit eine häufig festzustellende begriffliche und terminologische konfusion vermeiden. Daneben sind die Probleme zu diskutieren, die 
sich bei der Entwicklung dieses Begriffsbildungsverfahrens einstellen. Die in diesem ersten Teil der Arbeit getroffenen Aussagen sollen dann anhand der deadjektivischen Derivation des Russischen exemplarisch auf ihre Anwendbarkeit und Fruchtbarkeit hin überprüft werden.

Ad (2) Der empirische Teil

2.1. Der klassifikatorische Teil

Der klassifikatorische Teil bildet in zweierlej Hinsicht die Grundlage für die empirische Untersuchung der deadjektivischen Derivation:

(1) Um den empirischen Teil der Arbeit mit der Beantwortung der Frage abrunden zu können, ob, wie es theoretisch durchaus denkbar ist, von allen Elementen des Redeteils 'Adjektiv' Angehörige desselben oder anderer Redeteile als abgeleitet angenommen werden können oder ob eine Differenz zwischen den potentiell ableitenden und den tatsächlich ableitenden Adjektivtypen besteht, ist die Gesamtheit der Adjektive des Russischen aus Gründen der Systematik in bestimmte Teilmengen zu zerlegen. Als Einteilungskriterium dienen dabei verallgemeinerte Explikationen der nichtgrammatischen Inhaltselemente.

(2) Auf derselben Grundlage werden im AnschluB daran auch die Angehörigen der deadjektivischen Redeteile behandelt. Dies geschieht, um zu den Inhalten zu gelangen, die von den später unter anderem zu beschreibenden Kombinationen der Ausdrucksmittel bezeichnet werden.

\subsection{Der analytische Teil}

Ausgangspunkt ist hier die Kritik daran, dab in den bisher vorliegenden Arbeiten zur Wortbildung des Russischen, soweit mir bekannt, implizit lediglich für die Affixe eine bedeutungsunterscheidende bzw. bedeutungstragende Funktion angesetzt wird. Andere Mittel zum Ausdruck der Derivationsbeziehung bzw. - richtung bekommen a priori einen untergeordneten stellenwert zugewiesen, indem es heiBt, zwischen den Affixen und den phonematischen sowie den akzentuellen Variationen, die sich beim Vergleich des ableitenden wortes mit dem abgeleiteten Wort feststellen lassen, bestehe ein Kausalzusammenhang derart, daB die genannten Alternationen durch das jeweilige Affix 'hervorgeru- 
fen' würden. Um nachweisen zu können, daB eine solche Auffassung wenig fruchtbar ist, sind in diesem analytischen Teil alle Ausdrucksmittel, über die die russische Sprache verfügt, um in dem untersuchten Bereich die abgeleiteten Wörter von den ableitenden zu unterscheiden, unabhängig voneinander zu beschreiben. Das heibt also, daB a priori alle in Frage kommenden Ausdrucksmittel als 'gleichberechtigt' angesehen werden. Erst später, im funktionellen Teil, erfolgt die Bestimmung ihrer jeweiligen funktionellen Belastung.

\subsection{Der synthetische Teil}

Nachdem die für die deadjektivische Wortbildung des Russischen konstitutiven Inhalts-und Ausdruckselemente je für sich behandelt worden sind, soll festgestellt werden, welche Kombinationen von Ausdrucksmitteln theoretisch denkbar sind sowie welche dieser Kombinationen das Russische tatsächlich ausnutzt.

\subsection{Der funktionelle Teil}

Im AnschluB an die Darstellung aller in dem hier interessierenden Bereich verwirklichten Kombinationen der Ausdruckselemente sollen Aussagen über die funktionelle Belastung der Ausdrucksmittel getroffen werden. Zur Beantwortung der Frage, ob die bei der deadjektivischen Derivation eingesetzen Mittel in der genannten Hinsicht gleichwertig sind oder nicht, sind diese hinsichtlich ihrer unterschiedlichen identifikatorischen bzw. diskriminatorischen Potenz zu klassifizieren.

Ad (3) Kritische Würdigung der gewählten Heuristik

Im letzten Kapitel der Arbeit soll es darum gehen, die Leistung der 'analytisch-synthetisch-funktionellen' Methode auf dem Gebiet der Wortbildung zusammenfassend zu würdigen und die Ergebnisse mit denen zu vergleichen, die mittels dieser Heuristik im Rahmen der Formenbildung erzielt wurden. 
1. THEORE TISCH-BEGRIFFSKRITISCHER TEIL

Dem ersten und innerhalb der vorliegenden Arbeit besonders wichtigen Kapitel sei ein $\mathrm{Zitat}$ vorangestellt, das, wie noch zu zeigen sein wird, kaum an Aktualität eingebüBt hat: "Allgemein bekannt ist, dab die Entstehung eines jeden neuen Wissensgebietes unvermeidlich mit einer genaueren Bestimmung seines Untersuchungsgegenstandes verknüpft ist, d.h. mit einer Festlegung derjenigen Objekte korreliert, die in seinen Zuständigkeitsbereich fallen. Dieser ProzeB hat mit einer Präzisierung der für das entsprechende Wissenschaftsgebiet charakteristischen Begriffe und mit der Ausarbeitung einer speziellen Terminologie einherzugehen. Für den Bereich der Wortbildung geschah dies bisher noch nicht oder doch nur in einzelnen seiner Aspekte" (KUBRJAKOVA 1965, 11).

\subsection{Die Stellung der Wortbildung}

Zahlreiche Veröffentlichungen sind der Erörterung des Problems gewidmet, ob die Wortbildung eine selbständige Position unter den linguistischen Teildisziplinen einnehme oder ob sie einer der letzteren zuzuordnen sei. Eine Diskussion dieser Frage erübrigt sich in unserem Zusammenhang; gleichwohl soll in aller Kürze fegtgehalten werden, daB die Wortbildung in dieser Arbeit als ein autonomes Gebiet linguistischer Forschung aufgefaBt wird (vgl. dazu bspw. ZEMSKAJA, KUBRJAKOVA (1978, 113)). 'Autonom' bedeutet hier allerdings nicht, dab es keine Verbindungslinien zu anderen Bereichen der Linguistik gäbe (etwa zur Phonologie, zur flexionsmorphologie, zur Lexikologie oder zur Syntax), sondern lediglich, daB der Wortbildung ein besonderer Untersuchungsgegenstand zugrunde liegt, durch dessen Spezifik sie sich von allen anderen Disziplinen unterscheidet. Abgelehnt wird jedenfalls die Auffassung einiger der generativen Richtung verhafteten Sprachwissenschaftler, die linguistische Einteilung 2.8. in Lexikologie, Morphemik, Formen- und Wortbildung sei ein Relikt überkommener wissenschaftlicher Traditionen.'

1 "Генеративный подход к словообразованио слособствует преодоленио устарев- 


\subsubsection{Der Gegenstand der Wortbildung}

Was den Gegenstand der Wortbildung sowie das methodische Vorgehen bei dessen Beschreibung anbelangt, differieren die Meinungen der in diesem Bereich repräsentativen Russisten oft nicht unerheblich voneinander. Genau wie im Abschnitt 1.1. kann es auch hier nicht um eine eingehende Erörterung oder gar abschliebende Würdigung der verschiedenen Ansätze gehen; vielmehr sollen exemplarisch und damit notwendigerweise vereinfachend nur die allerwichtigsten Anschauungen zu diesem Problem vorgestellt werden.

Zunächst sei dabei kurz das Verhältnis von Synchronie und Diachronie in diesem Bereich angesprochen. Bis zu Beginn der fünfiger Jahre waren Wortbildung und Diachronie eng miteinander verbunden, da die 'Gesetze der Bildung von Wörtern' als den inneren Gesetzen der Sprachentwicklung untergeordnet aufgefabt wurden, die wiederum "mittels einer Untersuchung solcher Verfahren sichtbar werden und sichtbar gemacht werden können, bei denen auf der Grundlage bestimmter Wortbildungselemente neve Elemente entstehen, die von den ersteren sbgeleitet sind" (VINOGRADOV 1952, 123).2

Diese einseitige Sicht der Dinge hat bereits recht früh $A$. 1. SMIRNICKIJ in Frage gestelit, indem er ganz explizit auf die Aufgabe verwies, die die Synchronie bei der Erforschung der Wortbildung als eines Systems zu spielen habe. Allerdings sieht auch er die Diachronie noch in einer führenden Rolle, während er der Synchronie mehr oder weniger eine Hilfsfunktion zuweist: "Die Veränderung einer beliebigen sprachlichen Einheit geschieht nicht als Veränderung einer isolierten Einheit, nicht als isoliertes faktum, sondern als teil des Systems. Folglich ist die Linie der Synchronie, d.h. die des einzig existierenden Systems, bei der Erforschung von Sprachuerände-

(1) แих представлений $\infty$ отделенности уровней в структуре язына, а в целом аналитических и статических воззрений на языК." (ZASORINA 1972, 68). Die hier den 'Nichtgenerativisten' unterstellte Annahme von einer absoluten Autonomie der genannten Gebiete dürfte sich kaum beweisen lassen.

2 Vgl. dazU auch LEVKOVSKAJA $(1954,3)$ : "Боль山инСтвО нСвыХ слОВ [...] обылто образуется при помоци словообразовательныХ средств, тесно связанных со структурой языка и внутренними занонвми его развития." 
rungen, d.h. bei der diachronen Analyse, unbedingt zu berücksichtigen" (SMIRNICKIJ 1954, 16). 3

Die bis heute in ähnlicher form allgemein akzeptierte Abgrenzung eines historischen Wortbildungsprozesses und einer Wortbildung als System von in der Sprache funktionierenden 'strukturellen formen von Wörtern' hat als einer der ersten $K$. A. TIMOFEEV (1960) getroffen. ${ }^{4}$

Die folgenden Darlegungen werden sich ausschließlich auf die synchrone Wortbildung beziehen, innerhalb derer man gewöhnlich mehrere Aspekte unterscheidet. Zuvor sei jedoch darauf hingewiesen, daB oft nicht überprüft werden kann, auf welche Weise die Termini der in diesem Abschnitt (1.1.1.) zitierten Autoren mit den entsprechenden begrifflichen Korrelaten zusammenhängen. Auf eine kritische Auseinandersetzung soll jedoch vorläufig verzichtet werden, da diese sinnvoll nur dann erfolgen kann, wenn der eigene Begriffsapparat 'steht', eine Voraussetzung, die an dieser Stelle noch nicht erfüllt ist.

Während einige Linguisten die Analyse der Ausdrucksseite besonders hervorheben, indem sie sagen, die Wortbildung habe sich mit den materiellen Mitteln zu befassen, die das abgeleitete Wort vom ableitenden differenzieren (vgl. bspw. ZEMSKAJA 1973, 10), ist die Mehrzahl der Forscher sich darin einig, dab es mindestens zwei Aspekte zu untersuchen gilt, nämlich

a) die "Ableitungsstruktur von Wörtern (wie die fertigen Wörter gebildet sind)" und

b) den "WortbildungsprozeB (wie die Wörter gebildet werden)" (MOISEEV 1968, 74). Zu den Vertretern dieser Auffassung gehören neben zahlreichen anderen V.V. LOPAIIN und I.S. ULUCHANOV (1978, 40), V.I. MARKOV und G.A. NIKOLAEV $(1976,4 \mathrm{f})$ sowie K.A. TIMO-

3 Diese unsaubere Trennung von Synchronie und Diachronie ist auch in neuerer Zeit noch keineswegs überwunden. Ein ausgezeichnetes Beispiel dafür sind die Arbeiten von N.M. SANSKIJ, in denen der Autor bisweilen einer strikten Unterscheidung der Ebenen das Wort redet, sie jedoch in seinen empirischen Untersuchungen häufig miteinander vermischt. Vgl. dazu auch die berechtigte Kritik von PANOV (1970) an ŚANSKIJ (1968).

4 Vgl. auch ZEMSKAJA $(1973,6)$ : "Синхронное словообразование изучает отношения сосуиествуоиих единиц, диахрониеское - процессы тревранения одних едини в другие." 
FEEV ( 1966,1$)$. Wieder andere möchten nicht nur den strukturellen und den prozessualen Aspekt berücksichtigt wissen, sondern bringen zusätzlich auch eine funktionale Komponente ein (vgl. z.B. DOKULIL 1968; SEREBRENNIKOV 1972, 350; ZEMSKAJA, KUBRJAKOVA 1978, 113).

Ganz allgemein macht sich die Tendenz bemerkbar, den sogenannten prozessualen Aspekt deutlicher in den Vordergrund zu rücken. So beklagt bspw. ĊEREPANOV (1972, 43f): "In erheblich geringerem MaBe [als die sogenannte klassifizierende und die analytische Wortbildung - J.k.] ist der Bereich der dynamischen Wortbildung erforscht - die Gesamtheit der mit der synthetisierung von Wortbildungseinheiten verknüpften Fragen und Probleme[...], der Bereich, der letztendlich die Antwort auf die Frage "wie werden Wörter gebildet" geben muB."

Bereits L.V. ŠČ́RBA suchte die Wortbildung als linguistische Disziplin auf diesen Aspekt zu beschränken. S Mit fast identischem Wortlaut vertritt auch ARUTJUNOVA $(1961,3)$ diese Ansicht; vor allem aber handelt es oich um Vertreter der applikativ-generativen Richtung, die die Wortbildung als einen Prozeb verstanden wissen wollen, den es mittels des von ihnen entwickelten Verfahrens zu erklären gelte. "Das applikative generative Modell ist eine Sprachtheorie, die auf eine Erklärung der Beziehungen zwischen realen sprachlichen objekten in der Dynamik der Prozesse der Synchronie [d.h. im sogenannten Netz der tiefenstrukturellen Beziehungen einer Sprache - J.K.] abzielt" (GLEJBMAN 1972, 213). Bisweilen wird als 'Argument' für diese Betrachtungsweise angeführt, andernfalls erweise sich die Wortbildung als "bar einer Bildung von Wörtern" (MOISEEV 1968, 102). Aus der Versenkung hervorgeholt und aktualisiert werden neverdings auch wieder längst vergessen geglaubte Vorstellungen, nach denen dieser Aspekt der wissenschaftichen Erforschung der Wortbildung unmittelbar mit psychischen, kognitiven Prozessen korreliert werden müsse: "Also ist der

5 "Оаним из [...] основных отделов грамматикя являотся [...] правила словообразования, т.е. вопрос о том, как мохно делать новье слова. Вопрос mе о том, кан сделаны тотовые слова, - дело словаря, пде далина быть дана делимость слова" (ŚCERBA 1945, 181). 
Gegenstand der prozessualen Wortbildung (der Onomasiologie) einmal der AbleitungsprozeB, der psychische ProzeB, in dessen Verlauf sich das Ideelle lexikalisch materialisiert und objektiviert sowie zum anderen das Ableitungsmodell, in Ubereinstimmung mit dem sich dieser ProzeB realisiert. Als Untersuchungsobjekt konstituiert das Ableitungsmodell jenes Original, mittels dessen sich Affinitäten des Erkenntnismodells aufspüren lassen" (TOROPCEV 1980, 12f).

Dieser Minimalüberblick war notwendig, um folgende Feststellung treffen zu können: Die mit der Trennung von prozessualem und funktional-strukturellem Aspekt häufig implizierte Annahme einer Gegensätzlichkeit oder gar Unvereinbarkeit beider Bereiche ist nicht haltbar. Der primäre Untersuchungsgegenstand in diesem Bereich sind nun einmal die 'fertigen Wörter' (und zwar grundsätzlich alle) ${ }^{6}$, deren Strukturen es zu analysieren gilt. Erst nach diesem Schritt können Uberlegungen dahingehend angestellt werden, was mit diesen 'Wörtern' passiert iat bzw. was mit ihnen hätte passieren können. ${ }^{7}$ Die strukturel-

6 Fragen nach dem eigentlichen Analyseobjekt der Wortbildung werden selten gestellt, da in diesem Punkt weitgehende Ubereinstimung herrscht: "Принитиальное отличие дериватология от других областей науюи о языке заклонается в том, что она изучает не все слова язлка, а лишь слова производнње. При этом производные слова изучаится в дериваталогия не сами по себе, как отдельно взятые единиы лексической системы языка, а В их Отношеним К производящи словам" (NEMCENKO 1971, 26). - Eine solche Vorgehensweise ist methodisch einfach nicht haltbar, denn woher soll man letztendlich wissen, welche Wörter man sich als 'abgeleitet' und welche als 'ableitend' vorzustellen hat, wenn nicht zuvor prinzipiell alle Wörter der jeweils interessierenden Sprache einer von bestimmten Gesichtspunkten geleiteten Analyse unterzogen worden sind?

7 Der Problematik der Wortbildungssynthese ist auch eine Monographie von I.G. MILOSLAVSKIJ gewidmet, in der der Autor prinzipiell davon ausgeht, daB jegliche Verbindbarkeit bedeutungstragender Einheiten in erster Linie semantisch bedingt sei. Da jedes beliebige komplexe Zeichen nur dann gebildet werden könne, wenn ein entsprechendes Denotat existiere, das eine komplexe Nomination er laube, habe sich die Wortbildungssynthese mit den Regeln und den Restriktionen der Verbindbarkeit der bedeutungstragenden Einheiten auseinanderzusetzen; dies unter Berücksichtigung der semantischen Möglichkeiten, über die die betreffende Sprache im Rahmen der Wortbildung verfüge: "[ ... ] задача настоящей работы состоит В том, чтоби, имея индекс морфем русското языка с их значениями и фориальными преобразованиями, попьтаться отределить те правила, с помоцыо которых мапно, во первьх понимать членимые русские слова и, во вторьх, оорезовывать новые слова от данных по заданному семантинескому различио" (MILOSLAVSKIJ 1980, 4). 
le Analyse ist somit kein Selbstzweck, sondern eine Tätigkeit, ohne die auf dem Gebiet der Wortbildung 'nichts geht'.

Natürlich diente die Darstellung dessen, womit man sich im Rahmen der Wortbildung gewöhnich auseinandersetzt, auch dazu, den Boden für das in dieser Arbeit vorgeschlagene Verfahren zu bereiten, das alle wesentlichen Bereiche dieser Disziplin abdecken soll. Die empirische Analyse hat, wie bereits angekündigt, mit einer stringenten Begrifflichkeit zu operieren, um deren Erarbeitung es im folgenden Abschnitt (1.2.) gehen wird.

1.2. Sukzessive Einführung in die der empirischen Untersuchung zugrunde liegenden Begrifflichkeit

Die Notwendigkeit, der eigentlichen empirischen Untersuchung ein Kapitel voranzustellen, in dem vergucht werden soll, die grundlegenden Begriffe der Wortbildung als linguistischer Disziplin kohërent zu definieren, liegt nicht unbedingt von vornherein auf der Hand. Zu Recht könnte man fragen, ob es erforderlich sei, den zahlreichen Arbeiten, die sich mit Grundbegriffen der Wortbildung entweder in ihrer (wie auch immer verstandenen) Gesamtheit oder unter spezielien Gesichtspunkten auseinandersetzen, ein weiteres begriffliches 'Gebäude' gegenüberzustellen.

Der Begriff 'Gebäude' steht hier nicht von ungefähr, denn genau so, wie ein Bauwerk (in der Regel) sukzessive von unten nach oben, vom Einfachen zum Komplexen konstruiert wird, soll auch bei der nachfolgenden Konstruktion des begrifflichen Gerüsts vorgegangen werden. Bisher liegt nämlich speziell für das Gebiet der Wortbildung keine Arbeit vor, in der konsequent das Prinzip verfolgt würde, die Definitionen der entsprechenden Begriffe so anzulegen, daB diese entweder von vornherein nichtdefinierte Größen darstellen oder bereits in den entsprechenden vorangehenden Definitionen bestimmt wurden. 8 - Versuche, dieses Begriffsbildungsverfahren in unzusammenhängender

8 Hinsichtlich des Bereiches der Morphologie in ihrer Gesamtheit hat I.A. MKL'ĆUK (1975) einen beachtlichen Schritt in diese Richtung unternommen. 
Form durchzuführen, bringen leicht erhebliche Schwierigkeiten bei der Klassifikation der in empirischen Untersuchungen gewonnenen Beobachtungsdaten mit sich. In dieser Arbeit soll daher jeder Begriff zwar (soweit dies möglich ist) je für sich, doch ausschlieblich durch seine stellung zu den anderen für relevant erachteten Begriffen, d.h. nicht isoliert, definiert werden.

Prinzipiell nicht gesondert eingeführt werden alle Begriffe, die nicht unmittelbar fachsprachlicher Natur sind; explizit genannt, jedoch nicht definiert werden solche linguistischen Begriffe, die bereits hinreichend bestimmt sind oder als unter vielen (bzw. all ihren) Aspekten beschrieben gelten können.

\subsubsection{Nichtdefinierte Begriffe}

Zu den nichtdefinierten sollen hier die folgenden Begriffe zählen, zwischen denen zum Teil Relationen bestehen und die aus Gründen der leichteren Orientierung in aller Kürze erläutert werden:

1) Zeichen (Einheit (Gesamtheit) von Signifikant und Signifikat $)^{9}$

2) Signifikant (Ausdruck, der ein Signifikat bezeichnet)

3) Signifikat (Inhalt, der mittels eines Signifikanten ausgedrückt wird)

4) (natürliche) Sprache ((primäres) System von Zeichen)

5) Rede (Anwendung von Elementen des Zeichensystems zur Informationsvermittlung)

6) (konkreter) Text in der (natürlichen) Sprache (schriftlich fixierte Rede)

9 Die Auffassung, daB das Zeichen mindestens zwei Seiten aufweist, ist zwar allgemein akzeptiert, doch wird bisweilen auch eine triadische Relation angesetzt (bspw. zwischen 'Signifikant', 'Signifikat' und 'Syntaktik' bei MEL'ČUK (1975, 15; 1982, 40) oder zwischen 'Präsentation', 'Repräsentation' und 'Kommunikation' etwa bei BENSE (1971, 93), der damit eine pragmatische Komponente einbringt). 
7) Phonem (linear nicht weiter zerlegbares lautliches Sprachelement, das als selbständige Konstruktions- und Unterscheidungseinheit von Signifikanten dient)

8) prosodisches Merkmal (u.a. Betonung von Zeichen bei der Rede)

9) Kontext des Signifikanten (potentielle Umgebung des Signifikanten; mit anderen Worten, eine bestimmte Menge von Einheiten vom Iyp des Signifikanten, in Verbindung mit denen er vorkommen kann (vgl. MEL'ČUK 1975, 18))

10) Kontext des Signifikats (potentielle Umgebung des Signifikats; mit anderen Worten, eine bestimmte Menge von Einheiten vom Typ des Signifikats, in Verbindung mit denen es vorkommen kann)

11) Distribution des Signifikanten (Menge aller zulässigen Kontexte des Signifikanten (vgl. MEL'ĆUK 1975, 19))

12) Distribution des Signifikats (Menge aller zulässigen Kontexte des Signifikats) 10

Diese zwölf Begriffe bilden die Grundlage für die sich nunmehr anschließende Einführung in die spezifische Begrifflichkeit der Wortbildung als linguistischer Disziplin.

\subsubsection{Definierte Begriffe}

\subsubsection{Konkrete Wortform}

Prinzipiell hat die Analyse der Wortbildung einer Sprache von der Größe' (konkreter) Text in der betreffenden (natürlichen) Sprache' als oberster sprachlicher Einheit (vgl. DRESSLER 1970, 64) auszugehen, innerhalb derer es mittels bestimmter Prozeduren die eigentlichen Untersuchungsobjekte aufzufinden gilt. Da-

10 Im Gegensatz zu MEL'ĆUK, der 'Kontext' und 'Distribution' lediglich für die Ausdruckselemente gelten läßt, wird in der vorliegenden Arbeit (zumindest was den Bereich der Wortbildung anbelangt) davon ausgegangen, daß beide Parameter auch, um nicht zu sagen in erster Linie, gesondert für die Inhaltselemente zu gelten haben. 
bei sollen hier die mit der Textkonstitution verbundenen Probleme simplifizierend auBer Betracht bleiben.

Dieses zweiseitige 'Erstelement' konstituiert sich aus ebenfalls biplanen 'Sekundärelementen', den konkreten Wortformen. 11

Beispielsweise setzen sich die Texte

(1) Żena mużu plastyr', muż żene pastyr'.

(Die Frau ist ihrem Mann ein Pflaster, der Mann seiner Frau ein Hirt.)

sowie

(2) Drug moj, moj ruki pered edoj!

(Mein Freund, wasche die Hände vor dem Essen!) aus jeweils sechs, der Text

(3) Mčatsja tuči, v'jutsja tuci

(Es jagen die Wolken dahin, im Kreise die Wolken fliegen.)

dagegen aus vier konkreten Wortformen zusammen, die auf der graphematischen Ebene durch Leerstellen (blanks) im Schriftbild isoliert sind. 12

Wir wollen uns nun die frage vorlegen, ob man von bestimmten konkreten Wortformen unserer Texte sagen kann, sie seien in gewisser Hinsicht gleich. Mit dieser Uberlegung lösen wir uns bereits von der konkreten Manifestation, wir abstrahieren von ihr. Selbstverständich sind unsere Urteile bezüglich der Gleichartigkeit oder der Verschiedenheit zweier objekte jeweils von dem Aspekt abhängig, unter dem wir diese betrachten. Vom graphematischen Standpunkt (von der Ebene der Signifikanten) aus besehen, können wir in (2) die zweite und die dritte sowie in (3) die zweite und die vierte konkrete Wortform als identisch auffassen. Da wir jedoch für jede konkrete Wortform zwei Ebenen postulieren, und für uns Gleichartigkeit nur dann vorliegen soll, wenn sie beide Ebenen betrifft, gelangen wir zu

11 Vgl. ZALIZNJAK 1967, 20. - Andere mögliche Konstituenten unfangreicher Texte wie z.B. 'Absatz' oder 'Satz' sollen uns in diesem Zusammenhang nicht interessieren.

12 Das, was hier unter 'konkreter Wortform' verstanden wird, heiBt bspw. bei KUBRJAKOVA (1974, 15) 'Wort' und wird definiert als kleinste funktionale (= benennende) Spracheinheit, die in freier Verbindung auftreten kann. - Diese onomasiologische Funktion wird im Rahmen unserer Definition nicht eigens erwähnt, da sie natürlich zwangsläufig aus der angenommenen Zweiseitigkeit der 'konkreten Wortform' resultiert. 
dem SchluB, daB die in Rede stehenden konkreten Wortformen be $i$ (2) verschieden sind, weil der in beiden identische Signifikant jeweils für unterschiedliche Signifikate steht; hier haben wir es mit homonymen Wortformen zu tun. In (3) gelten für uns die betreffenden konkreten Wortformen als gleichartig, da wir annehmen, sie seien sowohl auf der Ebene ihrer Signifikanten als auch auf der ihrer Signifikate identisch.

\subsubsection{Abstrakte Wortform}

Jede Wortform, die im AnschluB an diese Identifikationsabstraktion in der genannten Hinsicht als mit einer anderen konkreten Wortform identisch oder nichtidentisch einzustufen ist, wird als Realisierung einer abstrakten Wortform aufgefaBt. 13 Wir können somit sagen, daB die Texte (1) und (2) jeweils sechs konkrete Wortformen enthalten, die je sechs abstrakten Wortformen zuzuweisen sind, während text ( 3 ) sich aus vier konkreten Wortformen zusammensetzt, die mit drei unterschiedlichen abstrakten Wortformen identifiziert werden.

1.2.2.3. Grammatische Bedeutung, nichtgrammatische Bedeutung, Grammem, grammatische Kategorie

Es war bereits mehrfach davon die Rede, dab man sich jede Wortform als biplane Einheit von Signifikant ( = bestimmte Konfigu-

13 Den Begriff der (abstrakten) Wortform hat schon V.V. VINOCRADOV ungefähr so verwendet, wie dies in der vorliegenden Arbeit geschieht: "Оавсловое единство слова сочетается с реальным или потенциальньм многооразием его форм. Формы слова - это разновидности одного и того пе слова, отличаюииеся друг от друга алементами морфалогического состава или синтактикофразеолопическими связями и соответствуюиими побочными оттенками. Пон-

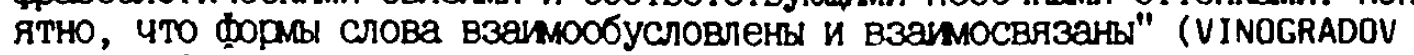
1944, 35). Im Abschnitt 'Morphologie' der AG-80 dagegen wird die in Rede stehende Einheit unter Zuhilfenahme des recht vagen $\mathrm{Kriteriums}$ der sogenannten 'allgemeinen kategorialen Bedeutung des Redeteils' definiert, indem gesagt wird, es handele sich bei (abstrakten) Wortformen um dem Sprachsystem angehörende morphologische Varianten der 'Wörter' eines gegebenen Redeteils, die neben der allgemeienen Bedeutung des Redeteils einen bestimmten Komplex sog. 'morphologischer' Bedeutungen (oder auch nur eine solche Bedeutung) trügen. 
ration von Ausdruckselementen) und Signifikat (= bestimmte Konfiguration von Inhaltselementen) vorzustellen hat. ${ }^{14}$ Somit wird angenommen, die integrierenden Bestandteile der Wortform seien der Wortformausdruck und der Wortforminhalt. Um (zunächst) auch für die letztgenannte Ebene deren Konstituenten angeben zu können, soll noch einmal auf den Beispielstext (1) zurückgegriffen werden.

'Zerlegt' man etwa die Hortformen żena, muzu, muzi, żene in ihre Phoneme, läßt sich ein Ergebnis beispielsweise folgendermaBen notieren:

$$
\mid z+e+n+a /, / m+u+i z+u /, / m+u+i /, / z+e+n,+e /
$$

In einem entsprechend umfangreichen Text des Russischen würden sich noch mehr (konkrete) Wortformen finden lassen, die mit den bereits genannten die Eigenschaft gemeinsam haben, daB sie jeweils hinsichtlich des einen (u.z. des ersten) Teils ihrer Signifikanten phonologisch miteinander übereinstimmen. Für die gewählten Beispiele ergäbe sich etwa dieses Bild:

$$
\begin{array}{rr}
|z+e+n,| \text { bzw. } / z+e+n /+\mid a / & |m+u+z /+| a / \\
/ e / & / u / \\
/ y / & / 0 / \\
/ u / & / e /
\end{array}
$$

Im folgenden wird davon ausgegangen, daß der veränderliche Teil von Wortformen deren grammatische Bedeutung ausdrückt und der leil, der (vereinfachend) als unveränderlich gelten kann, deren nichtgrammatische Bedeutung.

Vorläufig soll demnach in bezug auf Wortformmengen wie die oben bezeichneten, deren Angehörige sich durch die skizzierten Eigenschaften (i.d.R. Teilbarkeit in einen festen und in einen substituierbaren Bestandteil) auszeichnen, ein Inhaltselement dann grammatisch heiBen, wenn es (im beschriebenen Sinne) austauschbar ist und mit mindestens einem weiteren, in dieser Hinsicht gleichartigen Inhaltselement in einer solchen Beziehung steht, dab bei jeder einzelnen innerhalb eines Textes vorkom-

14 Hier und im folgenden wird unter dem nicht näher spezifizierten Terminus 'Wortform' stets die 'abstrakte Wortform' verstanden. 
menden Wortform der jeweiligen Wortformmenge eines dieser Inhaltselemente vorkommen muB. 15

Jedes einzelne grammatische Inhaltselement soll Grammem heiBen, während jede Gesamtheit von in bestimmter Hinsicht gleichartigen Grammemen mit dem Terminus grammatische Kategorie belegt wird. 16

1.2.2.4. Lexem

In der vorliegenden Arbeit kann es natürlich nicht darum gehen, prinzipiell aus sämtlichen Texten des Russischen die in ihnen enthaltenen konkreten Wortformen zu exzerpieren und dann anhand dieser Liste mittels der Abstraktionsoperation zu den abstrakten Wortformen zu gelangen. Stattdessen werden wir im empirischen Teil der Untersuchung von dem Lexem (dem Lexikoneintrag) ausgehen.

Bei dem Lexem handelt es sich immer um eine abstrakte Größe, die definiert werden kann als Resultat der Identifizierung aller Wortformen, die sich hinsichtlich ihrer Signifikate durch identische nichtgrammatische Inhaltselemente auszeichnen ${ }^{17}$ und auf der Ebene ihrer Signifikanten (ihrer Phonemkonfigurationen) einander zumindest ähnlich sind. 18

15 Die z.B. bei MEL'ĊK $(1974,99)$ zusätzlich geforderten Eigenschaften der Universalität und der Regularität werden hier als sekundär aufgefabt, da man mit ihnen sinnvoll nur dann operieren kann, wenn gleichzeitig das Kriterium der Obligatheit erfüllt ist.

16 Vgl. dazu auch ZALIZNJAK $(1967,27)$. - Für das Russische lassen sich bspw. die Inhaltselemente 'Nominativ', 'Genitiv', 'Dativ', 'Akkusativ', 'Instrumental', 'Präpositiv' in bezug auf die (unten näher zu bestimmenden) Wortformklassen 'Substantiv' und 'Adjektiv' als einander gleichartige Grammeme auffassen, da sie in ihrer Gesamtheit die grammatische Kategorie 'Kasus' konstituieren.

17 Von den möglicherweise unterschiedlichen Ausprägungen der grammatischen Inhaltselemente wird somit abstrahiert. - Die allen Wortformen eines Lexems geme insamen nichtgrammatischen Inhaltselemente konstituieren die Bedeutung des Lexems.

18 Ohne das Kriterium der formalen Ähnlichkeit, auf das unten noch näher einzugehen sein wird, würden alle Wortformen mit einander unähnlichen Signifikanten aber identischen Signifikaten (d.h. alle Synonyma) ebenfalls zu einem Lexem vereinigt. - Bei der praktischen Analyse stellt sich das Problem der Synonymie allerdings nicht, da jeder Lexikoneintrag als genau ein Lexem angesehen wird. 
Unsere drei Beispielstexte (S. 15) wurden bereits hinsichtlich der in ihnen enthaltenen konkreten und abstrakten Wortformen charakterisiert; nun soll der Frage nachgegangen werden, welche Wortformen als Repräsentanten eines und desselben Lexems gelten können. In Text (1) werden wir die Wortformen żeng und zene sowie muzu und muż zu jeweils einem Lexem zusammenfassen, da sie den genannten Bedingungen genügen. Gleiches gilt für die beiden konkreten Repräsentationen der Wortform tuci in Text (3). In Text (2) dagegen steht jede einzelne Wortform für jeweils ein anderes Lexem. - Statt des Terminus 'Lexem' wird in dieser Arbeit auch der als inm synonym aufgefabte Terminus 'Wort' verwendet. 19

\section{$1 \cdot 2 \cdot 2.5$. Wortarten}

In einem nächsten Schritt soll es nun darum gehen, die Lexeme des Russischen unter dem Gesichtspunkt ihrer Wortartenzugehörigkeit einzuteilen. Wie die onderen hier behandelten Größen sind auch die Wortarten (die Redeteile) immer Konstrukte des Linguisten, die nicht an sich existieren, sondern das Ergebnis eines von bestimmten Gesichtspunkten geleiteten linguistischen Klassenbildungsverfahrens darstellen.

Im Zusammenhang mit der (grammatischen) Wortartenklassifi-

19 Auch die Einheit, die nach unserem Verständnis als Lexem (bzw. als Wort) aufzufassen ist, bekomnt bereits bei VINOGRADOV in etwa die gleichen Eigenschaften zugewiesen: "Единство слова организуется прелде всего его лексиюо-селантическим стеринем, который является обим у всех его форм. С этой точю зрения безрезлично, имеет ли слово одну, нескалько форм или орразует целуг парадипму форм" (VINOGRADOV 1944, 34). Ganz ähnlich lautet die Definition des Lexems im Kapitel 'Morphemik' der AG-80 (123, 128). Später, im Kapitel 'Morphologie' (453), wird vom 'Wort' gesagt, es handele sich bei ihm zum einen (als formal ausgedrücktes System seiner 'lexikalischen' Bedeutungen) um eine Einheit der Lexik und zum anderen (als System all seiner Formen mit ihren grammatischen Bedeutungen) un eine Einheit der Grammatik. Auch bei MASLOV $(1972,7)$ gilt das Lexem sowohl als Einheit des lexikalischen wie des grammatischen 'Sprachsystems'. Dieser Gedanke eines quasi obligaten Vorliegens beider Arten von Inhaltselementen kann bisweilen sogar zu der Auffassung führen, eine bestimnte Einheit nur dann als Lexem zu interpretieren, wenn in ihr gleichzeitig nichtgrammatische und gramatische Bedeutungen repräsentiert sind (vgl. etwa SUNIK 1963, 37). 
kation bedient man sich für gewöhnlich oreier Kriterien, die meist nicht isoliert, sondern in bestimmter Kombination Berücksichtigung finden: des morphologischen, des syntaktischen und des semantischen.

Dem morphologischen Kriterium liegt in der Regel der Cedanke zugrunde, solche Lexeme zu einem Redeteil zusammenzufassen, deren Wortformen hinsichtlich der in ihnen repräsentierten grammatischen Kategorien gleichartig sind. Das syntaktische Kriterium fragt nach den funktionalen Positionen, die die Wortformen im Syntagma einnehmen können, und Grundlage des semantischen Kriteriums ist der "verallgemeinerte abstrahierte Bedeutungsgehalt (der die unmittelbare konkrete Semantik des Wortes überlagert)" (ADMONI ${ }^{3} 1970,62$ ).

Durch eine parallele Berücksichtigung aller orei genannten Kriterien zeichnet sich 2.B. die AG-70 (304) aus, die einer kritischen Uberprüfung ihres Begriffsbildungsverfahrens nicht standhalten kann (vgl. dazu KEMPGEN 1981, 14lff). In der AG-80 werden insgesamt gar vier Kriterien zugrunde gelegt, u.z., erstens, die verallgemeinerte Bedeutung, "die von den lexikalischen und den grammatischen Bedeutungen aller wörter der gegebenen Klasse abstrahiert", sowie, zweitens, der "für jede dieser Klassen charakteristische Komplex grammatischer Kategorien", ferner, drittens, ein "identisches Paradigmensystem", und schlieBlich, viertens, die "Gemeinsamkeit der fundamentalen syntaktischen funktionen" (AG-80, Bd. 1, 457). Allerdings werden die Prinzipien nicht eigens erläutert, die zur Auswahl gerade dieser Kriterien geführt haben.

Das syntaktische Kriterium könnte, für sich betrachtet, nur dann zu theoretisch akzeptablen Resultaten führen, wenn Begriffe wie 'Subjekt', 'Prädikat', 'Objekt' bereits ohne Rekurrenz auf die Wortarten definiert wären. Wir wollen annehmen, diese Voraussetzung sei in befriedigender Weise erfült, und uns nun der Proxis zuwenden. Einsetzproben würden uns, zumindest für das Russische, sehr schnell davon überzeugen, daB Wortformen verschiedener Lexeme eine und dieselbe funktionale Position im Satz einnehmen können, was wiederum zu einer Nichteindeutigkeit der Klassifikation führt, die uns vor weitere, vermutlich nur mit einem größeren Aufwand an zusätzlichen Annahmen und Bedin- 
gungen zu lösende Probleme stellt.

Was das semantische Kriterium anbelangt, so sind nach LEONT'EV (1971, 140) die "generalisierten semantischen Vorstellungen von den Wortarten [...] eine linguistische Fiktion, da sie nicht sprachunabhängig existieren und auch keine vorsprachliche psychische Kategorie darstellen". Wenn man den Uberlegungen LEONT'EVs folgen will, ist die sogenannte kategoriale Bedeutung als Kriterium schlicht unsinnig, da sie zunächst aus dem, was eigentlich gewonnen werden soll (aus den Redeteilen), zu extrahieren ist, um dann später als Zuordnungsgrundlage fungieren zu können. Hier liegt die Zirkularität klar auf der Hand. Doch spricht auch noch eine andere Erwägung gegen dieses Vorgehen: Der 'allgemeinen kategorialen Bedeutung' entspricht - zumindest im Russischen - kein spezielles korrelat auf der Ausdrucksseite von Wortformen, wohingegen es im Prinzip möglich ist, deren grammatische und nichtgrammatische Inhaltselemente analytisch voneinander zu trennen und ihnen die jeweiligen Ausoruckselemente zuzuordnen.

Das morphologische Kriterium macht sich bspw. KEMPGEN (1981) zunutze, um zu einer Wortartenklassifikation des Russischen $z u$ gelangen. Den primären Untersuchungsgegenstand bilden bei KEMPGEN die abstrakten Wortformen des Russischen, die er sich wie folgt gegeben denkt: Jede Wortform kann prinzipiell auf der Signifikantenebene als Konfiguration von Phonemen und auf der Ebene des Signifikats als oine Kombination nichtgrammatischer und gegebenenfalls grammatischer Bedeutungskomponenten beschrieben werden, wobei die letzgenannten "definitionsgemäß morphologisch, d.h. "am Wort" ausgedrückt" (KEMPGEN 1981, 162) sind.

Die Zusammenfassung der Wortformen zu Lexemen erfolgt auf der Grundlage der Stammemeinsamkeit, die wiederum (in der Regel) eine Gemeinsamkeit hinsichtlich der nichtgrammatischen Inhaltselemente signalisiert. Ein wichtiger Punkt innerhalb der KEMPGENschen Ausführungen ist der, daB die Begriffe 'Wortformmenge eines Lexems' und 'Paradigma dieses Lexems' nicht gleichgesetzt werden, da nicht alle Wortformen eines Lexems zusammengenommen auch in genau einem Paradigma vertreten sein müssen.

Welche Wortformen eines Lexems jeweils ein 'Kernparadigma' bilden, definiert KEMPGEN folgendermaßen: "(1) Zu jeweils einem 
Paradigma gehören alle diejenigen Wortformen eines Wortes, die sich voneinander nur durch die Ausprägungen einer oder mehrerer grammatischer Kategorien unterscheiden, nicht aber durch das Fehlen bzw. Vorhandensein solcher Kategorien" (ebd., 173).

Diese Bestimmung wird später dahingehend erweitert, daB auch solche Wortformen in das gemäß (1) gebildete Kernparadigma aufzunehmen seien, die dieses kombinatorisch ergänzen und bei denen hinsichtlich der in ihnen repräsentierten grammatischen Kategorien im Vergleich mit den bereits aufgrund (1) dem Kernparadigma zugeordneten Wortformen ein Inklusionsverhältnis derart vorliegt, dab sie genau eine Kategorie weniger aufweisen: "(2) Zusätzlich werden alle diejenigen Formen einem Paradigma nach (1) hinzugefügt, die zu diesem eine "kombinatorische Ergänzung" darstellen, unter der Voraussetzung, daB sich die Differenz bezüglich der vertretenen Kategorien auf genau eine beschränkt" (ebd., 176).

Mit (2) sollen aus ganz bestimmten Gründen unerwünschte (vgl. ebd., 174-176) Restmengen bei der Zerlegung eines Lexems in seine Wortformen ausgeschlossen werden.

"Praktisch betrachtet wurde das Material, das verschiedene Arbeiten zur russischen Grammatik präsentieren, in einem ersten Schritt daraufhin überprüft, welche formen von den einzelnen Wörtern überhaupt bildbar sind; danach war dann zu prüfen, welche Inhaltsbeschreibungen diesen formen gerecht werden" (ebd., 162).

Auf dieser Grundlage gelangt KEMPGEN zu 24 sogenannten Inhaltsparadigmen, denen jedes Wort des Russischen (jedoch nicht immer eindeutig) zugeordnet werden kann und die sich jeweils beschreiben lassen als Kombination einer nichtgrammatischen Bedeutung mit den Grammemen der grammatischen Kategorie( $n)$

(1) Kasus 20

20 In diese Klasse fallen 34 Wörter, "deren nichtgrammatische Bedeutung für sich [...] eine Zahlbedeutung zwischen "3" und "900" ausmacht" (ebd., 186), ferner die "9 sog. Kollektivzahlwörter von "2" bis "10"" (ebd., 187) sowie die Wörter "скально, Сталько, нескальюо und палтораста. Von den traditionell "Pronomen genannten Wörtern gehören gleichfalls ein Teil in diese Klasse, z.В. Себя, КТО, ЧТО, deren Kombinationen mit юое-, -ТО, -нибудь und -либо, ferner никто und ничто, Некого und Heyero" (ebd.). 
(2) Kasus und Numerus 21

(3) Kasus, Numerus und (im Singular) Genus 22

(4) Kasus und Genus 23

(5) Kasus, Numerus und (mask.) Genus

(6) Kasus, Numerus und (fem.) Genus

(7) Kasus, Numerus und (neutr.) Genus 24

(8) (mask.) Genus

(9) (fem.) Genus

(10) (neutr.) Genus 25

(11) Kasus, Numerus und (im Singular) Genus sowie Steigerung (Superlativ) 26

(12) Numerus und (im Singular) Genus sowie Steigerung (Komparativ, Superlativ) 27

Inhalt sparadigma

(13) repräsentieren solche Wörter, in denen ausschließlich nichtgrammatische Inhaltselemente ausgedrückt sind. 28

Bei KEMPGEN schlieBen sich an dieser stelle elf Inhaltspa-

21 "Diese Inhaltsparadigmen realisieren nur genau zwei wörter, nämlich die sogenennten "Personalpronomen der 1. und 2. Person", also Я/Mb [...] und $\mathrm{TW} / \mathrm{Bd} "$ (ebd., 188)

22 "Dieses Inhal tsparadigma wird [...] von dem "Personalpronomen der 3. Person", d.h. OH (OH, OHa, OHO, OHY im Nom.) realisiert" (ebd., 192).

23 "Dieses Inhaltsparadigma realisieren die Lexeme Два, Оба und полтора" (ebd., 194).

24 In die Klassen (5) bis (7) fällt "das traditionell so genannte Substantiv" (ebd., 195), außerdem "einige "Zahlwörter", wie Thсяча, миллион, половина, треть, десяток, сотня, nicht dagegen die "indeklinablen Substantive", sie bilden eigene Klassen" (ebd., 196).

25 Die Klassen ( 8 ) bis (10) werden von den 'indeklinablen Substantiven' realisiert (vgl. ebd., 208ff).

26 Hierher gehören 'Adjektive' mit "attributiven "Langformen" des Positivs" (ebd., 211), ferner "ОДИН [...] und alle Ordnungszahlwörter, angefangen mit nepвый" (ebd., 214), außerdem die ""Pronomen" мОЙ, ТВОЙ, СВОЙ, ТОТ, весь, чей, некй, ничей" (еbd.) sошіе "Lexeme wiе таковой, каковой" (ebd., 215).

27 In diese Klasse fallen die "ausschließlich prädikativ gebrauchten "Kurzformen"" (ebd., 213) der 'Adjektive' und "Lexeme wie [...] далreh, ТОТОВ, нymeH etc." (ebd., 215).

28 Dieses "Inhaltsparadigma realisieren die traditionellen Präpositionen, Konjunktionen, die eigentlichen Adverbien, $[\ldots]$ des weiteren Wörter wie да $[\ldots]$, тде $[\ldots]$, вдвоем $[\ldots]$, нельзя $[\ldots]$ u.a." (ebd., 216). 
radigmen an, die den Bereich der Verbalwortformen abdecken. Für die Zwecke dieser Arbeit genügt es, lediglich die beiden 'Infinitivinhaltsparadigmen' zu nennen, da es sich bei den ihnen zuzuweisenden 'Verben' um Einheiten in der form handelt, wie sie auch im Lexikon begegnet.

Bei Inhaltsparadigma

(14) handelt es sich um die Kombination einer nichtgrammatischen Bedeutung mit den Grammemen 'Aktiv', 'unvollendeter' oder 'vollendeter Aspekt' sowie 'nichtreflexives Genus'.

Inhal tsparadigma

(15) Unterscheidet sich vom vorhergehenden lediglich durch das Grammem 'reflexives Genus' (anstelle des Grammems 'nichtreflexives Genus') (vgl. ebd., 221).

Mit der Aufstellung dieser Inhaltsparadigmen hat KEMPGEN die Objektmenge geschaffen, auf deren Basis er in einem nächsten Schritt die eigentliche, numerisch-taxonomisch ausgerichtete Wortartenklassifikationdes Russischen vornimmt. Eine Darstellung dieses recht komplexen Verfahrens wijde hier zu weit führen und ist auch nicht notwendig, da für die Zwecke der vorliegenden Arbeit folgendes gesagt werden kann:

Innerhalb der empirischen Untersuchung werden solche Lexeme, deren Wortformen das Inhaltsparadigma (11) realisieren, in inrer Funktion als Basis für die Ableitung anderer Wörter untersucht, deren formen entweder ebenfalls in das Paradigma (11) eingehen oder aber anderen Paradigmen angehören. Da jedoch nicht sämtliche Wörter, die diese Voraussetzung aufgrund der Bedingungen KEMPGENs erfüllen (insbesondere nicht die in Anm. 26 neben den Adjektiven mit attributiven Langformen des Positivs angeführten), in der vorliegenden Arbeit als ableitende Lexeme behandelt werden sollen, ist folgende Einschränkung zu machen: Zum Redeteil 'Adjektiv' zählen ausschlieblich solche Lexeme, deren Wortformen das genannte Inhaltsparadigma vollständig realisieren.

Die Wörter, deren formen die Paradigmen (5) bis (10) konstituieren, sollen in folgenden 'Substantive' heiBen, und als 'Verben' gelten solche Lexeme, deren Wortformen unter anderem den Paradigmen (14) oder (15) angehören. 
Als problematisch erweist sich in Zusammenhang mit der Wortbildung lediglich die Behandlung der Einheiten, die dem Inhaltsparadigma (13) zugewiesen wurden. 29 Theoretisch ist es nämlich durchaus möglich, sich auch Lexeme mit diesem Inhaltsparadigma als von Adjektiven abgeleitet zu denken. 30 Aus diesem Grunde ist es legitim, sich hier eines 'Kunstgriffs' zu bedienen, mittels dessen man den in Rede stehenden Wörtern doch noch 'beikommen' kann.

Gedacht ist hier an ein Verfahren von GASPAROV (1971), der das Problem der 'formalen Wortklassen' des Russischen unter Zugrundelegung der funktionalen Eigenschaften zu lösen versucht, die die verschiedenen Redeteile im Syntagma annehmen ${ }^{31}$ : "Wir gehen [...] von der Annahme aus, dab diese Eigenschaften innerhalb des Textes in der Kollokation der Wörter hervortreten, im Charakter ihrer Verteilung" (GASPAROV 1971, 65).

Auf die Frage, wie GASPAROV in einem ersten Schritt die Lexemgrenzen zieht, sei hier nicht eingegangen. Auch soll es nicht darauf ankommen, die Spezifika des Klassenbildungsverfahrens näher zu erläutern. 32

Die Grundüberlegung bei diesem zweiten Schritt ist die, daB die Wortformen jedes Lexems notwendig Relationen zu anderen Einheiten anzeigen. [...] Anders gesagt, die Auswahl eines der

29 Infolge seines anders gearteten Erkenntnisinteresses stellt sich das Problem einer weiteren Spezifizierung innerhalb dieser Gruppe für KEMPGEN nicht, da er seine Arbeit ausdrücklich u.a. als "Grundlagenstudie" (KEMPCEN 1981, VII) für eine weitergehende Beschäftigung mit der formenbildung begreift.

30 Die neben den sogenannten 'Vollwörtern' außerdem in diese Klasse fallenden 'Funktionswörter' werden von vornherein aus der Betrachtung ausgeschlossen, da es sich bei diesen um solche Lexeme handelt, die in erster Linie zur Herstellung syntagmatischer Beziehungen dienen und für sich betrachtet nur mit erheblichen Einschränkungen als Zeichen im obigen Sinne gelten können.

31 Dieses funktional-distributionelle Verfahren ist (trotz der genannten Mängel) im Zusammenhang mit den Wörtern, um die es hier geht, am geeignetsten, da das morphologische Kriterium in diesen Fällen nicht 'greift' und das semantische aus den erwähnten Gründen überhaupt abzulehnen ist.

32 Der Autor begründet seine Vorgehensweise in GASPAROV 1971 sowie ausführlicher in CASPAROV 1975. Zur Kritik an diesem Verfahren vgl. KEMPGEN 1980, S3ff, 165f, 171f, $179 \mathrm{ff}$. 
Paradigmenangehörigen des ersten Elements bedingt die Auswahl des entsprechenden Paradigmenangehörigen des zweiten Elements" (ebd., 66). In dem uns interessierenden Bereich (Inhaltsparadigma (13) bei KEMPGEN) gelangt CASPAROV zu folgenden Ergebnissen, die für die Zwecke dieser Arbeit übernommen werden sollen.

Innerhalb der sogenannten Nominalprädikate unterscheidet GASPAROV neben 'formalen Klassen', die sich dem Inhaltsparadigma (12) bei KEMPGEN zuweisen lassen, auBerdem

(1) "Wörter, die ausschlieblich in unpersönlichen Konstruktionen gebraucht werden - unpersönlich-prädikative Wörter oder Prädikative: marb [(es ist) schade], rpex [(es ist) schändlich], nopa [(es ist) Zeit]" (ebd., 91).

(2) Wörter wie грустно (traurig), хорошо (gut), тихо (sti11), die in unpersönlichen Konstruktionen "im Singular der neutralen form stehen und die Korrelation mit den anderen Genus- und Numerusformen verlieren" (ebd.)

Bei den unter (1) und (2) angeführten Lexemen handelt es sich um Angehörige solcher formalen Klassen, "die durch eine spezifische Verwendung in unpersönlichen Konstruktionen charakterisiert sind, U.z. durch eine Neutralisierung des Paradigmas (indem sie sich hinsichtlich Genus, Person und Numerus nicht unterscheiden) sowie durch eine Zustandsbedeutung, die zu ihrer kategorialen Bedeutung wird" (ebd., 92). - Heshalb CASPAROV ausgerechnet an dieser Stelle die 'kategoriale Bedeutung' ins Spiel bringt, bleibt unklar.

Nicht zu den Nominalprädikaten gehören

(3) "Adverbiale Adverbien. Unter dieser bedingten Benennung kann man [...] Umstandsadverbien zusammenfassen, die fähig sind, bei einem Verb zu stehen, sich jedoch nicht mit einem Nomen verbinden" (ebd.).

(4) 'Adnominale Adverbien' wie много (viel), гораздо (bedeutend), значительно (beträchtlich), die nicht mit Angehörigen der formalen Klassen 'Verb' und 'Infinitiv', dafür aber mit Angehörigen der Klasse 'Adjektiv (in der Form des Komparativs)' kollokieren.

Obwohl die Inhaltsparadigmen nicht mit den traditionellen Wortarten gleichzusetzen sind, da, wie bereits erwähnt, der Um- 
fang der Lexeme nicht immer mit dem des Paradigmas identisch zu sein braucht, kann für die Zwecke der vorliegenden Arbeit wie oben dargestellt vorgegangen werden, was damit zusammenhängt, daB sich das angeschnittene Problem in bezug auf die im Rahmen des zu beschreibenden Ausschnittes aus der Wortbildung interessierenden Wort(form)mengen so nicht stellt.

\subsubsection{Flexionsendung, Wort formstamm}

Bisher wurde von der Wortform immer in ihrer Gesamtheit gesprochen. Als Voraussetzung für die empirische Untersuchung soll es nun darum gehen, innerhalb der Wortform gewisse cliederungen derart vorzunehmen, daß sich etwa angeben läBt, mit welchen Inhaltselementen die Ausdruckselemente korrelieren.

So werden in der $A G-70$ alle grammatischen und nichtgrammatischen Inhaltselemente des Wortes letzten Endes durch den Begriff der 'flexionsendung' bestimmt, der selbst dagegen praktisch undefiniert bleibt. Stattdessen heibt es einfach, die Flexionsendung sei Exponent der grammatischen Kategorien Genus, Kasus, Numerus und Person ( $A G-70,31 \mathrm{f}$ ).

In der AG-80 holt man etwas weiter aus, indem von den fle$x$ ionsendungen gesagt wird, 8 handele sich bei ihnen um solche 'Morphe', deren wechselseitiger Austausch innerhalb von Wortformen zu einer Veränderung der 'morphologischen' Bedeutungen des Genus, des Numerus, des Kasus und der Person führe. Ferner wird angemerkt, die 'Flexionsmorphe'besetzten die finale Position einer Wortform, sofern auf sie nicht die 'Morphe'

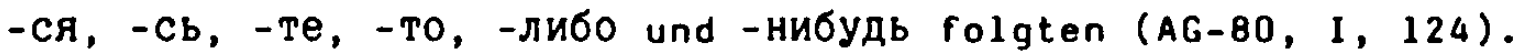

In diesen beiden Standardwerken (und nicht nur hier) gilt die Flexionsendung offensichtlich als etwas ganz Selbstverständliches, das keiner speziellen Anslyse und Definition bedarf. Gelegentlich wird jedoch der Versuch unternommen, genouer zu bestimmen, worum es sich bei der flexionsendung handelt. Dabei geht man dann in aller Regel von dem Kriterium der Position und/oder von dem der grammatischen funktion aus.

Das positionelle Kriterium ist für eine exakte Bestimmung der Flexionsendung wenig hilfreich, da diese innerhalb der Wort- 
form nicht eindeutig distribuiert ist und keinen festen platz einnimmt.

So unterscheidet z.B. MILOSLAVSKIJ (1980, 233) die folgenden Distributionen und Positionen (Unterstreichungen von mir J.K.):

"1) nach dem Stamm am Wortende (доM-â);

2) nach dem Stamm vor einem Flexionselement (скаa-h-Te) [In der vorliegenden Arbeit gilt das, was hier von MILOSLAVSKIJ 'Flexionselement' genannt wird, als 'Derivationselement'.];

3) nach dem Stamm vor einem Derivationselement (MO-ㅁ- $\mathrm{Cb}$ ) [Sofern man, wie in der vorliegenden Arbeit, geneigt ist, $-\mathrm{Cb},-\mathrm{Cg}$ als Derivationselemente zu begreifen.];

4) nach einem flexionselement am Wortende (ид-и-те);

5) nach einem flexionselement vor einem flexionselement (стро-ящ-й̆-ся дом);

6) nach einem flexionselement vor einem Derivationselement (бeper-u-Te-cb)."

Was das Kriterium der grammatischen funktion anbelangt, so wurde von $F . F$. FORTUNATOV und seiner Schule vorgeschlagen, nur solche Einheiten zu den flexionselementen zu zählen, die zur Herstellung von Relationen im Text dienen. Implizit wird diese Auffassung auch bei ŚVEDOVA (1966, 53) vertreten: "Flexionsendungen werden als Morpheme verstanden, die grammatische Bedeutung tragen und deren gegenseitiges Austauschen immer mit einer Veränderung der syntaktischen Kollokation des Wortes verknüpft ist." Bei einer solchen Sicht auf die Dinge kommt man in Schwierigkeiten, wenn man etwa angeben soll, um was für Einheiten es sich handelt, die beispielsweise die Grammeme der Numeruskategorie beim Substantiv oder die der Tempuskategorie beim Verb ausdrücken. Die Ersetzung eines dieser Grammemedurch ein anderes derselben grammatischen Kategorie verändert zwar unter Umständen die syntagmatische (nicht die syntaktische) Position der entsprechenden Wortform, doch signalisiert sie etwas über eine Veränderung im Rahmen der auBersprachlichen Wirklichkeit. Diesen, in der vorliegenden Arbeit aufgrund inrer Obligatheit 'grammatisch' genannten Elementen, eignet also auch eine eigentlich 'nominative' Funktion (zum Verhältnis von 
'nominativer' und 'syntaktischer' Bedeutung vgl. ZALIZNJAK $1967,23 f)$.

In dieser Arbeit soll daher unter der flexionsendung eine solche Phonemkonfiguration innerhalb der Wortform verstanden werden, die in bezug auf das Paradigma, dem die Wortform angehört, eine (meist aber mehrere) obligate Bedeutung(en) ausdrückt. - Die hier beschriebene Aufgabe können im Paradigma eines Lexems auch materiell nicht realisierte 'Leerstellen' (ఐ) erfüllen, die mit den entsprechenden phonematisch ausgedrückten flexionsendungen anderer Lexeme desselben Redeteils funktional korrelieren.

An dieser Stelle sind wir bei einer Operation angelangt, deren Voraussetzungen oft nicht hinterfragt werden und deren Konsequenzen infolgedessen häufig umstritten sind. Gemeint ist die Prozedur zur Auffindung der Flexionsendungen und anderer Phonemkonfigurationen innerhalb einer Wortform, von denen man annimmt, sie repräsentierten bestimmte Inhaltselemente.

Bei einem solchen Verfahren wird in der Regel zwischen beiden Sprachebenen implizit eine gewisse Symmetrie vorausgesetzt, die es gestatten soll, bestimmte, zu heuristischen Zwecken notwendige Segmentierungen vorzunehmen. Die Grenzen der Segmente mit bestimmtem Inhalt und bestimmtem Ausdruck macht man durch einen Vergleich formal und semantisch miteinander korrelierender Wortformen ausfindig. Die gerade angenommene symmetrische Beziehung zwischen Inhalts- und Ausdrucksebene nutzend, geht man davon aus, daB die Selbständigkeit eines Segments innerhalb der Wortform mittels der Ersetzungsprobe feststellbar sei: Wenn die Substitution eines Segments der Ausdrucksseite durch ein anderes Segment derselben Ebene eine Veränderung auf der Inhaltsseite bewirkt, dann wird zwischen diesem Segment und dem übrigen Teil der Wortform eine Grenze gezogen.

Solch ein Vorgehen ist selbstverständlich nur dann möglich, wenn man sich zwei weitere theoretische Prämissen zu eigen macht: 1) Minimale Spracheinheiten mit einer Ausdrucks- und einer Inhaltsseite sind obligatorisch diskret. 2) Die Wortform besteht aus einer Abfolge von solchen Einheiten, die nach bestimmten Verknüpfungsregeln organisiert sind.

Eine Betrachtung konkreten sprachlichen Materials zeigt sehr 
schnell, daß sich mit den genannten Prämissen nicht immer erfolgreich operieren läbt, da sie lediglich aus dem wunsch entstanden sind, eindeutige Segmentationen zu erhalten. Dieses traditionelle Vorgehen bei der Analyse führt in der Regel zwar zu diskreten Einheiten, die sich dann wieder zu einer Ganzheit kombinieren lassen, doch begibt man sich auf diese Weise häufig der Möglichkeit, den spezifischen Zusammenhang der Elemente innerhalb der Wortform zu erkennen.

In den eingangs ( $S .2$ ) genannten Arbeiten zur formenbildung slavischer Sprachen sind die Prämissen der Symmetrie und der Diskretheit aufgegeben worden, was den Blick für die besonderen Korrelationen der Ausdrucks- und der Inhaltselemente freigemacht hat. Zur Verdeutlichung soll hier nur ein Beispiel angeführt werden. Betrachten wir dazu die Wortform Bumy (ich sehe), bei der wir die flexionsendung -y ansetzen wollen, da wir davon ausgehen, dab sie Träger der grammatischen Bedeutungen 1. Person, Singular, Indikativ, Präsens, Aktiv ist. In phonologischer Notation erhalten wir $[v, i z-u] .33$ Können wir nun aber davon ausgehen, die Phonemkonfiguration vor -y habe nichts mit dem Ausdruck der genannten Bedeutungen zu tun, oder anders: LäBt sich zwischen [ $v, i z ̇]$ und [u] eine eindeutige Grenze ziehen, indem man etwa sagt, [u] diene zum Ausdruck der grammatischen und [ $v, i z]$ zum Ausdruck der nichtgrammatischen und nur der nichtgrammatischen Bedeutungselemente der Wortform Bumy? Diese frage kann nur verneint werden, da $[v, i z]$ nicht nur Träger einer bestimmten nichtgrammatischen Bedeutung ist, sondern auch signalisiert, daB es sich bei der wortform jedenfalls nicht um die 2. oder die 3. Person Singular Präsens und auch nicht um die 1., die 2. oder die 3. Person Plural Präsens handelt. Das Segment /ž/ zeigt somit gleichzeitig zwei Inhaltselemente an. In gewisser Hinsicht ähnlich verhält es sich auch im Rahmen der Wortbildung, was unten noch ausführlicher zur Spra-

33 Bei den Einheiten, die hier und im folgenden in der mit eckigen $\mathrm{klam-}$ mern gekennzeichneten Transkription verwendet werden, handelt es sich um Phoneme. - Dabei werden, in Anlehnung an die Konzeption der 'Moskauer Schule', z.B. die Laute [y] und [i] als Vertreter eines starken Vokalphonems / $i /$ angesehen. 
che kommen wird.

Die genannten Einschränkungen im Auge behaltend, wollen wir nun darangehen, innerhalb der Lexeme, deren Wortformen die Inhaltsparadigmen (1) bis (7), (11) und (12) sowie (14) und (15) repräsentieren, die flexionsendungen festzulegen. 34 Im Zusammenhang mit der Wortbildung können wir uns auf recht einfache Operationen beschränken, da es nicht notwendig ist, alle Wortformen des Lexems einer Prüfung zu unterziehen. Dies liegt darin begründet, daß bej der empirischen Untersuchung die jeweils interessierenden Einheiten immer nur in der form berücksichtigt werden, wie sie im Wörterbuch repräsentiert sind, also in der sogenannten Grundform. 35

Für die Lexeme mit den Inhaltsparadigmen ( = IP) (1), (2) 36 , $(3)^{37}$ bis (7) sowie (12) gilt folgendes:

I Endet die Grundform auf einen Konsonanten, so ziehe eine Grenze nach diesem Konsonanten.

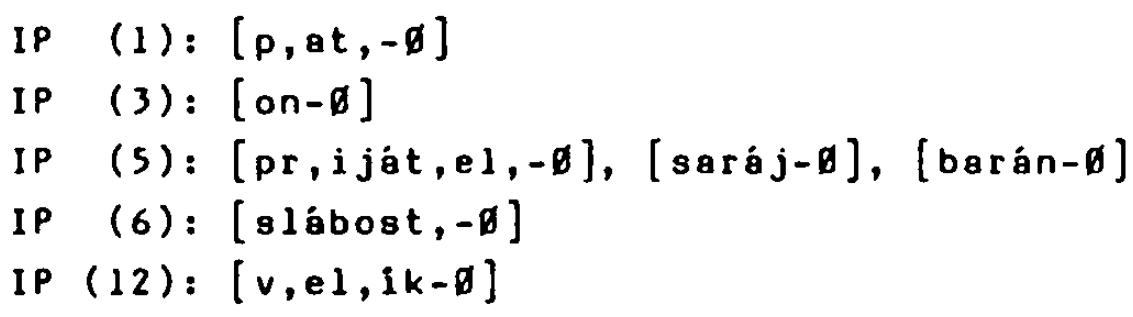

34 Die sogenannten indeklinablen Substantive (Inhaltsparadigmen (8) bis (10)) klamnern wir wegen der bei KEMPCEN (1981, 208ff) angesteliten Überlegungen aus der Betrachtung aus, merken jedoch an, daß dann, wenn man hier überhaupt Flexionsendungen enzusetzen geneigt ist, die nachstehenden Operationen I und II durchgeführt werden können.

35 Die formale Prozedur zur Festlegung der Floxionsendungen würde sich erheblich komplexer gestalten, wenn es unumgänglich wäre, alle Wortformen eines Lexems in dieser Hinsicht zu analysieren. Ein anschauliches Beispiel dafür ist der von ZALIZNJAK (1967, 112ff) entwickelte Algorithmus zur Auffindung der Flexionsendungen innerhalb der Nominalparadigmen des Russischen.

36 Nicht von den nachfolgenden Operationen betroffen werden die Lexeme 9 und TW, hinsichtlich derer hier die Position ZALIZNJAKs $(1967,113)$ übernommen werden soll: $[j a-g],[t i-g]$.

37 Die Wortformen, die statt des Grammens 'mask.' die Grammeme 'fem.' oder 'neutr.' der grammatischen Kategorie 'Genus' ausdrücken, fallen aus der Betrachtung heraus, da es sich bei ihnen nicht um Grundformen (im Sinne von 'Lexikoneintrag') handelt. Gleiches gilt hinsichtlich der Lexere mit dem Inhaltsparadigna (11). 
II Endet die Grundform auf einen Vokal, so ziehe eine Grenze $z$ wischen diesem Vokal und der vorangehenden Phonemkonfiguration der Wortform.

$$
\begin{aligned}
& \text { IP (1): }[s, e b,-a],[t r,-i],[p, a ́ t, e r-o],[k t-0] \\
& \text { IP (2): }[m-i],[v-i] \\
& \text { IP (4): [dv-a],[ób-a], [poltor-á] } \\
& \text { IP (6): [rabót-a],[séj-a], [stat,-á], [mužć, in-a] } \\
& \text { IP (12):[v,el,ik-á], [v,el,ik-ó] }
\end{aligned}
$$

In bezug auf die Lexeme mit dem Inhaltsparadigma (11) führe die nachstehende Operation durch:

III Endet die Basisform auf - แй, -й̆ oder auf-oй, so ziehe eine Grenze $z$ wischen diesen Segmenten und der vorangehenden Phonemkonfiguration der Wortform. $[k r a s, i v-i j],[s, i n,-i j],[k r, i v-o j]$

Für die Lexeme mit den Inhaltsparadigmen (14) und (15) schlieblich soll folgendes gelten:

IV Endet die Grundform auf - ть (-Ться), -ти (-тись) oder auf $-4 b(-4 b C 9)$, so ziehe eine Grenze zwischen diesen Segmenten und der vorangehenden Phonemkonfiguration der Wortform.

$[$ koló-t, $],[$ koló-t,-s,a]; $[v, e s-t, i],[v, e s-t, 1-s$,$] ;$ $[b, e r, \dot{e}-c],,[b, e r, \dot{e}-c,-8, a]$

Wir werden sagen, bei der Phonemkonfiguration links von der Grenze handele es sich um den Stamm der Wortform, der als Träger der nichtgrammatischen Inhaltselemente dieser Wortform fungiert und gleichzeitig signalisieren kann, welche grammatischen Inhaltselemente mit ihm nicht kompatibel sind. Weist eine Wortform keine Flexionsendung auf, so sind Wortform und Stamm dieser Wortform identisch.

\subsubsection{Wurzel der Wortform; Affix}

Im folgenden soll es darum gehen, innerhalb des stamms der Wortform gegebenenfalls weitere zeichenhafte Phonemkonfigurationen (die sogenannte 'Wurzel' und das sogenannte 'Affix') aufzufin- 
den. Wie die empirische Analyse zeigen wird, gelten die oben gemachten Einschränkungen hinsichtlich der Symmetrie und der Diskretheit sprachlicher Einheiten auch in bezug auf dieses Elemente. Gewöhnlich bedient man sich zur Bestimmung dessen, was eine 'Wurzel' ist, dreier Kriterien, die hier stellvertretend und exemplarisch an Aussagen verdeutlicht werden sollen, wie sie bei V.V. LOPATIN und bei I.S. ULUCHANOV zu finden sind.

1) Das Kriterium der Unbedingtheit. Die 'Wurzel' gilt als zentraler, für jede Wortform obligater Bestandteil (vgl. ŚVEDOVA 1966, 54; Russkij jazyk, 116; AG-80, I, 124). - Bei diesem obligatheitskriterium handelt es sich zwar um eine notwendige, jedoch zumindest theoretisch nicht gleichzeitig auch um eine hinreichende Bedingung, da nicht ausgeschlossen ist, daB auf diese Weise zusätzlich ein Element aus einer anderen Klasse ( $z . B$. aus der Klasse 'Affix') definitorisch zur Wurzel gezählt wird. Daher ist das Kriterium der obligatheit um das der 'Wurzelidentität' zu ergänzen.

2) Das formale Kriterium. Die 'Wurzel' wird als Hauptelement der nichtgrammatischen Bedeutung der Wortform angesehen, wobei von ihr gesagt wird,

a) sie habe eine konkretere Bedeutung als die anderen Elemente der Wortform (vgl. Russkij jazyk, 116) oder

b) sie repräsentiere deren "hauptsächliche, konkrete, 'gänzlich lexikalische' Bedeutung" (ŚVEDOVA 1966, 53) oder

c) sie trage eine "gegenständlich-logische, gänzlich lexikalische Bedeutung und gleichzeitig eine logischgrammatische" (LOPATIN, ULUCHANOV 1963, 191).

Abgesehen davon, dab es schwerfällt, zu begreifen, was sich hinter einer 'gegenständlich-logischen' Bedeutung verbirgt und wie man dazu kommt, ausgerechnet im Zusammenhang mit der 'Wurzel' auch eine sogenannte 'logisch-grammatische' Bedeutung (womit die 'kategoriale Bedeutung' gemeint sein dürfte) anzusetzen, gibt es Sprachen (darunter das Russische), bei denen Elemente aus der Klasse 'Wurzel' höchst abstrakte Bedeutungen tragen können. 
Neben dem formalen, dem semantischen und einem positionellen Kriterium (relativ freie Stellung der Wurzel innerhalb des Stamms) bringt JANKO-TRINICKAJA (1968, 534) einen quantitativen Aspekt ins Spiel, der in diesem Zusammenhang einen gangbaren Weg weist. Die Autorin entwickelt den Gedanken, daB die Aussonderung der 'Wurzeln' einer Sprache zu einer erheblich umfangreicheren Liste führen würde als die Aussonderung aller sonstigen in den Wortstämmen der gegebenen Sprache enthaltenen Elemente. - Der Haken bei der Sache ist nur der, daB nicht gesagt wird, nach welchen Prinzipien die Zuweisung der Elemente zu den entsprechenden Rubriken erfolgen soll. So ist der beschriebene Sachverhalt a posteriori zwar richtig; es bedarf jedoch der Operationalisierung, um diese quantitative Beziehung tatsächlich für unsere Zwecke nutzen zu können.

Eine solche Operationalisierung ist z.B. ANDREEV (1967) im wesentlichen gelungen. Ausgehend von der Annahme, daB hohe positionsabhängige Graphemhäufigkeiten am Anfang und am Ende von Wörtern das Vorhandensein eines Affixes anzeigen, entwickelt ANDREEV einen statistisch-kombinatorischen Algorithmus, in den Wörter mit mindestens vier Buchstaben eingegeben werden. Resultat dieser Prozedur ist das Paradigma der Affixe einer Sprache (eine ausführliche Beschreibung dieses Verfahrens geben AL TMANN, LEHFELDT (1980, 195-198)).

Im Prinzip den gleichen Weg wie JANKO-TRINICKAJA hat zur Lösung dieses Problems I.A. MEL'ĊUK (1975, $37 \mathrm{ff}$ ) beschritten. 38 Wie die genannte Autorin läBt sich nämlich auch MEL'C̈UK von der Grundüberlegung leiten, dab es prinzipiell möglich sei, eine Liste aller zeichenhaften Phonemkonfigurationen innerhalb der Wortstämme einer Sprache aufzustellen. Nur geht MEL'ČUK einen entscheidenden Schritt weiter, inden er jedem einzelnen Zeichen diejenigen Zeichen zuordnet, mit denen es innerhalb u $n d$ auBerhalb der Wortform potentiell kollokiert. Mit dieser Liste

38 Wie bereits in Anmerkung 9, S. 13 angedeutet, geht MEL'ĆUK von einem triplanen Zeichen aus, das neben der Ebene des Signifikanten und der des Signifikats noch eine syntaktische Ebene enthält, die hier vereinfachend als die Ebene charakterisiert werden soll, die Auskunft darüber gibt, mit welchen anderen Zeichen das jeweils gegebene Zeichen kollokieren kann. 
würden zwei quantitativ und qualitativ ungleiche Mengen von Zeichen erfabt: Eine erheblich größere Menge, von der gilt, daB die 'Syntaktik' ihrer Elemente Hinweise auf deren interwörtliche Kollokationsmöglichkeiten enthält, während sich die vergleichsweise geringe Restmenge dadurch auszeichnet, dab ihre Angehörigen ausschlieblich intrawörtliche Relationen eingehen. Die Zeichen der letztgenannten Gruppe sind zwar in der Lage, sich an der Herstellung interwörtlicher Beziehungen zu beteiligen, doch resultiert diese Fähigkeit nicht aus den Besonderheiten ihrer 'Syntaktik', sondern hängt ausschlieblich mit ihrer Signifikatebene zusammen. 39

Zusammenfassend läBt sich folgendes festhalten: Sowohl JANKOTRINICKAJA wie auch MEL'ČUK sind bestrebt, die Einheiten, die sie später 'Affixe' oder 'Wurzeln' nennen wollen, aufgrund ihrer quantitativen Charakteristika (aufgrund ihrer Systemfrequenz) als Klassen zu ermitteln. Beide gelangen (a posteriori) zu dem Ergebnis, daB die Klasse der 'Affixe' deutlich weniger Elemente als die der 'Wurzeln' enthält. Da diese Bedingung für sich allein noch nicht hinreichend ist (unklar bleibt, nach welchen Kriterien die eigentliche Klassenbildung erfolgen sol1), charakterisiert MEL'CUK die Angehörigen der Klasse 'Affix' als solche Einheiten, die sinnvoll nur mit Angehörigen der Klasse 'Wurzel' kollokieren könnten, während für die Angehörigen der Klasse 'Wurzel' der UmkehrschluB nicht gelte.

Do die genannte Beziehung zirkulär ist, wird in der vorliegenden Arbeit das quantitative Kriterium vernachlässigt und die 'Wurzel' lediglich als die zeichenhafte Phonemkonfiguration innerhalb des Wort(form)stamms aufgefaBt, welche solche Hinweise auf die interwörtlichen Kollokationsmöglichkeiten enthält, die nicht on ihrem Signifikat festgemacht sind.

Vom 'Affix' wird gewöhnlich gesagt, es handele sich bei inm

39 "Так, суфикс отглагальното суиествительното -ение, конечно суцественно воздействует на межсловную сочетасаость той словоформы, в ноторую Он входит: получение имет другую мексловную синтактияу непели палучить; тем не менее, это воздействие на синтактику словофориы палучение

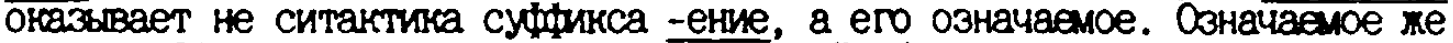
этого суфинса есть совокиность указаний о изменения части реии и модели улравления исходной основы" (МЕL'С́UK 1975, 38). 
um eine Einheit, die innerhalb der Wortform nicht obligat sei (vgl. AG-80, I, 124) und deren Fehlen die Einheit des Wortes als eines solchen nicht oufhebe (vgl. CARENKO 1979, 428). Ferner wird angeführt, das 'Affix' könne nicht mit dem Wortstamm identisch sein (vgl. Russkij jazyk, 116) und repräsentiere lediglich eine sogenannte auxiliare Bedeutung, die entweder 'lexikalischer' (vgl. JANKO-TRINICKAJA 1968, 334) oder 'kategorialer' (vgl. ŚVEDOVA 1966, 53) oder 'derivationeller' oder aber 'grammatischer' ('morphologischer') Natur sei (vgl. LOPATIN, ULUCHANOV 1963, 191; Russkij jazyk, 26; AG-80, 1, 124). - Wie oben bereits im Zusammentiang mit der 'Wurzel' angedeutet, durfte eine eindeutige Zumeisung all dieser Bedeutungen nur in Spezialfällen gelingen, nicht jedoch im allgemeinen fall.

Einen anderen Standpunkt nimmt ZEMSKAJA $(1966,4)$ ein, wenn sje nur solche Elemente als 'Affixe' betrachtet, "die in zwei oder mehr semantisch miteinander korrelierenden wörtern vorkommen, die jedoch verschiedene Wurzeln haben", und dabei nicht berücksichtigt, daB die sogenannten 'unikalen Affixe' dann aus der Betrachtung herausfallen mübten.

Hier soll unter einem 'Affix' eine solche zeichenhafte Phonemkonfiguration innerhalb des Wortformstamms verstanden werden, die niemals mit letzterem identisch ist und sinnvoll nur mit Zeichen vom Typ 'Wurzel' kollokieren kann. - In bezug auf inre Verbindbarkeit mit der Wurzel einer Wortform sind zum einen das vor der Wurzel stehende 'Präfix' sowie zum anderen das sich on die Wurzel anschlieBende 'Suffix' zu unterscheiden.

\subsubsection{Derivationsbeziehung}

Was die spezifische Terminologie der Wortbildung als linguistischer Disziplin anbelangt, der wir uns nun im wesentlichen zuwenden wollen, so wird in der vorliegenden Arbeit der Begriff der 'Derivationsbeziehung' als grundlegend angesehen, da er in dem Sinne primär ist, dab sich ous inm, unter Berücksichtigung der bisher eingeführten Größen, alle weiteren notwendigen Begriffe entwickeln lassen.

Die sogenante 'Wortbildungaderivation' betrachten unter an- 
deren auch LOPATIN und ULUCHANOV (vgl. Russkij jazyk, 304) sowie KUBRJAKOVA (in SEREBRENNIKOV 1972, 370) als den zentralen Begriff der synchronen Wortbildung. Andere Autoren nennen dagegen andere Einheiten, denen sie diese Schlüsselrolle zuweisen. Hier kann es lediglich darum gehen, diese Auffassungen in Auswahl und in aller Kürze vorzustellen: Bei DoKULIL (1962, 202) ist der sogenannte 'Wortbildungstyp' (als eine Gesamtheit, die sich konstituiert in der Identität (1) der onomasiologischen Struktur solcher Wörter, die dem Typ angehören, (2) des lexikalisch-grammatischen Charakters der betreffenden 'Wortbildungsstämme' und ( 3 ) der jeweiligen 'Formanten') der übergeordnete Begriff; bei KUBRJAKOVA nimmt in einer früheren Arbeit (1965, 24) das 'abgeleitete Wort' diese Position ein, und bei BARTOSEVIC (1972, 87) das 'Derivatem' als "von der 'parole' abstrahiertes allgemeines Bildungsschema never Wörter" (z.B. "gegenständliches Substantiv + uCT" (ebd.)). Ganz ähnlicher Meinung sind (ebenfalls in einer früheren Arbeit) LOPATIN und ULUCHANOV (1969, 120), in der sie den 'Formanten' als die zentrale Einheit ansehen, von der sie sagen, es handele sich bei ihr um ein "strukturelles Schema, das allen Bildungen eines Typs gemeinsam und folglich Träger der Wortbildungsbedeutung ist". - Alle diese Größen können nicht im oben genannten Sinn als primär gelten, da sie jeweils andere Einheiten der Wortbildung als bekannt voraussetzen.

Derivationsbeziehungen werden üblicherweise bei zwei oder auch mehr synchron nebeneinander existierenden wörtern angesetzt, von denen nicht nur gilt, dab sie die gleiche Wurzel aufweisen und von daher formal 40 wie auch inhaltlich ${ }^{41}$ in be-

40 "Отнопения производности [...] есть всегда фо р м а $\Omega$ в н х е н н е о т н о ше н и я, т.е. отношения производности по форие. [...] Нз этого следует, что отношения производности могут быть установлены [...] там, где они офориляются в пределах слова, с адной стороны, и лишь там, с другой, где единство корня в серия образований не вызыBQеT COMHеHИ"" (KUBRJAKOVA 1974, 184f).

41 "Словообрезовательная мотивация [...] - это отношение мепду двумя резными словами, обладагоиими следроиими признаками: I) оба слова именот один и тот же корень; 2) значение одного из них: а) палностыо входит в значение другото [...] или б) топдественно лексическам значенио друго, но Синтаксическое позици этих слов различны" (ULUCHAHOV 1971, 38). Diese 
stimmter Weise miteinander korrelieren, sondern darüber hinaus, dab sie in einem Abhängigkeitsverhältnis zueinander stehen. 42

Aufbauend auf diesen drei Kriterien macht KUBRJAKOVA (1974, $227 \mathrm{ff}$ ) einen konkreten Vorschlag zur Abgrenzung von derivationellen und anderen Beziehungen zwischen zwei Wörtern (oder den Wortformen eines Wortes). Dabei geht sie von der frage aus, ob bei der Ableitung der 'sekundären' Form der Stamm der 'primären' Form formale und/oder inhaltliche Veränderungen erfährt oder nicht und unterscheidet auf dieser Grundlage sechs fälle. 1) Wird der 'primäre' Stamm weder formal noch inhaltlich modifiziert, so liegt flexion (словоизменение) vor. Bsp.: [stol-g] (der Tisch) - [stol-á] (des Tisches).

Unbedingt anzumerken ist an dieser Stelle, daB (wie allein schon das Beispiel verdeutlicht: hier liegt eine von KUBRJAKOVA nicht gewürdigte Modifikation, nämlich Akzentwechsel, vor) diese grobe Simplifizierung lediglich Spezialfälle, nicht jedoch den allgemeinen Fall abdeckt. Doch selbst dann, wenn man davon ausgeht, daß KUBRJAKOVA nicht den allgemeinen Fall benennen und die 'restlichen' Fälle von den Punkten ( 3 ), (4) und ( 5 ) abdecken will, bleibt die Beziehung der einzelnen Punkte zueinender unklar, da lediglich das unter (4) Beschriebene gleichfalls ' словоизиенение' heißt. Das unter (3) und (5) Dargestellte fällt dagegen unter die Kategorie 'Форм00бpazoвarke' (F ormenbildung), was nach KUBRJAKOVA offensichtlich etwas anderes ist und nicht als Spezialfälle von (1) aufgefaßt werden soll. Diese Sicht der Dinge ist gedanklich kaum nachzuvollziehen, da das hier als '\$opmoo6pazosarue' Bezeichnete ganz sicher nicht über den Rahmen des 'croвоизменение' hinausgeht.

2) Wird der 'primäre' Stamm zwar nicht formal, jedoch in bezug auf seine (nichtgrammatischen) Inhaltselemente verändert, so haben wir es mit der Wortbildung (словообразование) oder genaver, mit der sogenannten Transposition zu tun. Bsp.: [zólot-o] (Gold) - [zolot-ój] (golden).

Hier wird ebenfalls nicht überzeugend argumentiert, da man durchaus den

(41) Definition ist (zumindest was $2 b$ ) anbelangt) Nicht von vornherein evident, da es Wörter mit gemeinsamer Wurzel, gleicher Bedeutung und gleicher syntaktischer Position gibt, die sich zunächst eirmal durchaus als in einer Ableitungsbeziehung stehend denken lassen (vgl. etwa uиpb und широта). Die inhaltlich gleiche Argumentation findet sich im übrigen auch in der AG-80 ( 1,133$)$.

42 "Отношения синхронной производности наблоданотся у слов с обии корнем там и тогда, когда одно слово (основу) можно рассматривать как базируюпееся на другом (цругой), как оуусловленное этим други словом, когда из двух взаммосвязанныХ однокорневьх единй одна [...] источник поропдения, а другая - его результат" (KUBRJAKOVA in SEREBRENNIKOV 1972, 371). 
Standpunkt verteten kann, der jeweilige Stamm sei in bezug auf seine nichtgrammatischen Inhaltselemente keineswegs berührt. Darüber hinaus zeichnet sich das von KUBRJAKOVA gewählte Beispiel, genau wie das vorangehende, durch einen Akzentwechsel aus, d.h., auf der formalen Ebene wird etwas hinsichtlich der auszuwählenden grammatischen Inhaltselemente signalisiert.

3) Wird der 'primäre' Stamm formal, nicht aber inhaltlich verändert, so liegt formenbildung ( $u . z$. 'morphologische Stammbildung') vor. Bsp.: [d,élaj-u] (ich mache) - [d,éla-l] (machte ).

Auch hier (genau wie in den fällen (5) und (6) signalisiert die formale Veränderung bestimmte grammatische Inholtselemente, weshalb der Aussage KUBRJAKOVAs in dieser Form nicht zugestimnt werden kann.

4) Wird der 'primäre' Stamm sowohl formal wie auch in bezug auf seine grammatischen Inhaltselemente verändert, so handelt es sich um flexion. Bsp.: wir geben - wir gaben.

5) Wird der 'primäre' Stamm sowohl formal wie auch in bezug auf seine (nichtgrammatischen) Inhaltselemente modifiziert, und ist dieser inhaltliche Unterschied für eine ganze Reihe von Bildungen eines und desselben 'Typs' konstant und vorhersagbar, so liegt Formenbildung vor. Bsp.: Steigerungsstufen der Adjektive.

6) Wird der 'primäre' Stamm sowohl formal wie auch in bezug auf seine (nichtgrammatischen) Inhaltselemente verändert, und ist dieser inhaltiche Unterschied innerhalb einer ganzen Reihe von Bildungen eines und desselben Typs nicht immer konstant und vorhersagbar, so hat man es mit Wortbildung zu tun. Bsp.: krasnet' (rot werden), belet' (weiB werden) aber choroset' (hübscher werden; bei chorosij (gut)), durnet' (häblich werden; bei durnoj (schlecht)).

Positiv ist zu vermerken, daB es sich hier um einen der ganz wenigen Versuche handelt, mögliche Relationen zwischen Wörtern bzw. zwischen Wortformen aufgrund bestimmter Kriterien zu differenzieren.

Berücksichtigt man jedoch die vorgebrachten Kritikpunkte, dann stellt sich heraus, daB das von KUBRJAKOVA vorgeschlagene Verfahren wenig geeignet scheint, die geforderte Abgrenzung zu leisten, da die Unterschiede hinsichtlich der einzelnen Arten von Beziehungen zwischen Wörtern und Wortformen keineswegs ols immer so eindeutig gelten können, wie dies bei KUBRJAKOVA dar- 
gestellt ist. Darüber hinaus ist es als wenig sinnvoll zu erachten, bei der Bestimmung dessen, worum es sich bei einer 'Derivationsbeziehung' handelt, gleich auch das Kriterium der 'Derivationsrichtung' einzubeziehen, da dieser Schritt logisch doch erst als zweiter getan werden kann. Zunächst ist festzulegen, von welchen Wörtern man sagen will, sie stünden in einer Ableitungsbeziehung zueinander; erst wenn dies geschehen ist, hat es Sinn, von 'primären' und 'sekundären' Einheiten zu reden. Daher erscheint es vorteilhaft, bei der Definition dessen, welche Wörter man als in einer Derivationsbezeihung stehend auffassen kann, zunächst einmal von der absoluten Gleichwertigkeit der jeweils untersuchten Einheiten auszugehen.

So verfährt z.B. VOLOCKAJA, die in bezug auf solche Wortpare, die 1) eine gemeinsame Wurzel aufweisen, 2) verschiedenen Redeteilen angehören (wodurch Wortbildungsbeziehungen zwischen wörtern eines und desselben Redeteils von vornherein aus der Betrachtung ausgeschlossen werden) und sich 3 ) bei verschiedenem Präfix durch ein und dasselbe Suffix auszeichnen oder umgekehrt (verglichen werden beispielsweise pare wie izvleć' (herausziehen) - izvlečenie (Herausziehen) und privlec' (heranziehen) - privlećenie (Heranziehen), nicht aber izvleć privlecenie ${ }^{43}$ ), ein Verfahren anwendet, das "eine Prozedur darstellt, die aus der sukzessiven Ausführung der folgenden Handlungen besteht: 1) es werden mit jedem der zu prüfenden Wörter als Bestandteil sinnvolle Wortverbindungen ausgewählt, die dann 2) nach bestimmten Regeln umzubilden sind" (VOLOCKAJA 1960, 101). Dabei gelten zwei Wörter dann hinsichtlich aller ihrer (nichtgrammatischen) Bedeutungen als in einer Derivationsbeziehung stehend, "wenn die gewählte sinnvolle Wortverbindung weder mit dem einen noch mit dem anderen zu prüfenden Wort nach der Anwendung einer vorgegebenen Transformation unsinnig uird" (ebd., 105f).

Das Gesagte sei an einem kurzen Beispiel, u.z. am Wortpaar

43 Bei diesem und in ähnlichen Fällen handelt es sich auch aufgrund der in der vorliegenden Arbeit befolgten Prinzipien um nichtkorrelative bortbildungsbeziehungen, da zwischen den Inhaltsseiten dieser beiden Lexeme eine Intersektionsrelation gegeben ist. 
složnyj (schwierig) - složnost' (Schwierigkeit) erlüutert. Für sloznyj wählt VOLOCKAJA die folgenden Kontexte aus: sloznyj vopros (schwierige Frage), složnaja zadaća (schwierige Aufgabe), složnoe dokazatel'stvo (schwierige Beweisführung). Dann wendet sie eine sogenannte Transformation an, die in diesem Fall aus zwei Schritten besteht. 1) Ersetze sloznyj durch sloźnost'! 2) Verwende den Kontext jeweils in der form des Genitivs! Als Ergebnis erhält VOLOCKAJA složnost, voprosa (Schwierigkeit der Frage), složnost' zadaci (Schwierigkeit der Aufgabe) und sloznost' dokazatel'stva (Schwierigkeit der Beweisführung). Áhnliche Uberlegungen stellt auch GINZBURG (1978) an, nur ist bei inm das Verfahren noch unklarer formuliert.

Die hier zur Bestimmung dessen, welche Wörter als in einer Ableitungsbeziehung befindlich gedacht werden können, vorgenommene Verknüpfung von Wortbildung und Syntagmatik ist zwar prinzipiell erfolgversprechend, doch noch längst nicht in dem Maße ausgereift, als daB sie generell widerspruchsfreie Ergebnisse zu liefern geeignet wäre. Die Auswahl der jeweiligen Kontexte muB als höchst subjektiv gelten, zumal letztere immer so modelliert werden können, daß das jeweils gewünschte Resultat auch tatsächlich eintritt.

Was unter einer Derivationsbeziehung zu verstehen ist, läbt sich auf elegantere und eindeutigere Art und Weise bestimmen, wenn man zunächst die Relationstypen, die zwischen jeweils zwei (prinzipiell aber auch mehr) Wörtern bestehen können, ouflistet und erst dann angibt, wo sich Wortbildungs-oder Ableitungsbeziehungen ansetzen lassen. Einen solchen Apparat, von dem gesagt werden kann, er sei in der Lage, das Geforderte zu leisten, hat MEL'CUK (1968, 427f) unter Zugrundelegung bestimmter mengentheoretisch aufgefabter Beziehungen zwischen den Inholts-und den Ausdrucksseiten sprachlicher Zeichen entwickelt. 44

Aus der Uberlegung heraus, dab man sowohl die Ausdrucksseite (A) wie auch die Inhaltsseite (I) von Lexemen ala Konfigurationen bestimmter Phoneme bzw. bestimmter Inhaltselemente auffas-

44 Die Ausführungen MLL'ĊUKs bilden auch bei JACHNOW (1978, 13ff) die Grundlage für eine Beschreibung dessen, was als Ableitungsbeziehung aufzufassen ist. 
sen können, setzt MEL'ĆUK zwischen zwei Lexemen (genauer gesagt. zwischen den Stämmen zweier Lexeme) $L_{1}$ und $L_{2}$ in bezug auf ihre Ausdrucks- und ihre Inhaltsseiten jeweils eine der folgenden Relationen an:

1) Die Identitätgrelation $: A\left(L_{1}\right)=A\left(L_{2}\right), I\left(L_{1}\right)=I\left(L_{2}\right)$

2) Die Inklusionsrelation $: A\left(L_{1}\right) \subset A\left(L_{2}\right), I\left(L_{1}\right) \subset I\left(L_{2}\right)$

3) Die Intersektionsrelation $: A\left(L_{1}\right) \cap A\left(L_{2}\right) \neq D$,

4) Die Exklusionsrelation $I\left(L_{1}\right) \cap I\left(L_{2}\right) \neq \emptyset$ : $A\left(L_{1}\right) \cap A\left(L_{2}\right)=\emptyset$, $I\left(L_{1}\right) \cap I\left(L_{2}\right)=\emptyset$

Indem er diese vier Beziehungstypen ouf beiden Ebenen miteinander kombiniert, gelangt MEL'Zuk zu siebzehn unterschiedlichen formal-inhaltlichen Relationen, die nun im einzelnen aufgelistet werden sollen.

(1) $A\left(L_{1}\right)=A\left(L_{2}\right), I\left(L_{1}\right)=I\left(L_{2}\right)$

Bsp.: ostrov (Insel) - ostrov (Insel)

(2) $A\left(L_{1}\right)=A\left(L_{2}\right), I\left(L_{1}\right) \subset I\left(L_{2}\right)$

Bsp.: višnja (Kirsche) - višnja (Kirschbaum)

(3) $A\left(L_{1}\right)=A\left(L_{2}\right), I\left(L_{1}\right) \cap I\left(L_{2}\right) \neq D$

8sp.: pogremuska (Kinderklapper) poqremuśka (Schelle (am Schlagzeug))

(4) $A\left(L_{1}\right)=A\left(L_{2}\right), I\left(L_{1}\right) \cap I\left(L_{2}\right)=\emptyset$ Bsp.: sajka (Bande (von Leuten) - sajka (Kübel)

(5) $A\left(L_{1}\right) \subset A\left(L_{2}\right), I\left(L_{1}\right)=I\left(L_{2}\right)$

Bsp.: 1isa (Fuchs) - 1isica (Fuchs) 45

(6) $A\left(L_{1}\right) \subset A\left(L_{2}\right), I\left(L_{1}\right) \subset I\left(L_{2}\right)$

Bsp.: dom (Haus) - domik (kleines Haus)

(7) $A\left(L_{1}\right) \subset A\left(L_{2}\right), I\left(L_{1}\right)>I\left(L_{2}\right)$

Bsp.: lingvist (Fachmann auf dem Gebiet der Linguistik) Iingvistika (Linguistik)

(8) $A\left(L_{1}\right) \subset A\left(L_{2}\right), I\left(L_{1}\right) \cap I\left(L_{2}\right) \neq \theta$

Bsp.: moskvić (Einwohner von Moskau + männl. Geschlecht) moskvickka (Einwohner von Moskau + weibl. Geschlecht)

45 Zu diesem Typ kam man auch Beziehungen wie палучить - получениe zählen (die MEL'ČUK im übrigen unter (1) einordnet). 
((9) $A\left(L_{1}\right) \subset A\left(L_{2}\right), I\left(L_{1}\right) \cap I\left(L_{2}\right)=\varnothing$

Bsp.: most (Brücke) - mostovaja (Fahrdamm, Pflaster)

(110) $A\left(L_{1}\right) \cap A\left(L_{2}\right) \neq \emptyset, I\left(L_{1}\right)=I\left(L_{2}\right)$

Bsp.: pedantičeskij (pedantisch) pedantićnyj (pedantisch)

(111) $A\left(L_{1}\right) \cap A\left(L_{2}\right) \neq B, I\left(L_{1}\right) \subset I\left(L_{2}\right)$

Bsp.: opponirovat' (opponieren)

opponent (einer, der opponiert)

(112) $A\left(L_{1}\right) \cap A\left(L_{2}\right) \neq \emptyset, I\left(L_{1}\right) \cap I\left(L_{2}\right) \neq \emptyset$

Bsp.: nožke (kleiner $F u B)$ - nožisča (groBer fuB)

(113) $A\left(L_{1}\right) \cap A\left(L_{2}\right) \times \nabla, I\left(L_{1}\right) \cap I\left(L_{2}\right)=\emptyset$

Bsp.: boginja (Göttin) - bogatyj (reich)

$(114) A\left(L_{1}\right) \cap A\left(L_{2}\right)=\varnothing, I\left(L_{1}\right)=I\left(L_{2}\right)$

Bsp.: samolet (flugzeug) - gèroplan (flugzeug)

(115) $A\left(L_{1}\right) \cap A\left(L_{2}\right)=\emptyset, I\left(L_{1}\right) \subset I\left(L_{2}\right)$

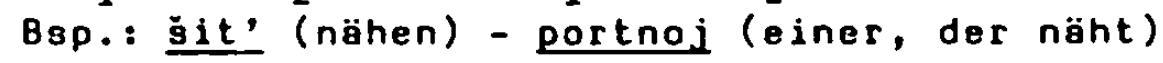

(116) $A\left(L_{1}\right) \cap A\left(L_{2}\right)=1, I\left(L_{1}\right) \cap I\left(L_{2}\right) \neq \emptyset$

Bsp.: Zerebec (Pferd + männl. Geschlecht) -

kobyla (Pferd + weibl. Geschlecht)

(117) $A\left(L_{1}\right) \cap A\left(L_{2}\right)=0, I\left(L_{1}\right) \cap I\left(L_{2}\right)=\emptyset$

Bsp.: moloko (Milch) - zevtra (morgen)

Bevor es nunmehr darum gehen soll, die einzelnen Beziehungstyypen näher zu analysieren, sei darauf hingewiesen, dab in der vuorliegenden Arbeit nur solche Relationen als 'echte' Derivat tionsbeziehungen aufgefabt werden, von denen gilt, dab sie sBich bei den jeweils in Rede stehenden Wörtern in bestimmter, nnoch näher zu erläuternder Hinsicht ouf der Inhalts-wie auch auf der Ausdrucksseite manifestieren, d.h., dab sie die entspprechenden Zeichen in ihrer Ganzheit berühren. Somit wird hier djie gängige Auffassung geteilt, daB "in der Sprache Wortbilduungsbeziehungen am Kreuzungspunkt der Beziehungen einer formalden und einer inhaltlichen Derivation entstehen" (KUBRJAKOVA inn SEREBRENNIKOV 1972, 373).

Von den siebzehn Relationstypen werden wir für unsere Zwekkke sagen, insgesamt acht von ihnen repräsentierten bestimmte, allerdings unterschiedlich zu beurteilende Derivationsbeziehun- 
gen. Bei der Mehrzahl von ihnen handelt es sich um solche Beziehungen, die hier 'nichtkorrelativ' genannt werden sollen, da sie lediglich eine der beiden Seiten des Lexems als Zeichen berühren. Hierzu zählen die Relationen (2) und (15), die sich rein inhaltich begründen sowie die Relationen (5) und (9), bei denen lediglich die Ausdrucksseite tangiert ist. Einzig bei den Relationen (6), (7) und (11) liegen 'korrelative' oder 'echte' Derivationsbeziehungen vor, da beide Seiten der jeweiligen Lexeme betroffen sind. Dieser Umstand gilt zwar auch für Beziehungen vom Typ (8), doch sollen, wie bereits an anderer Stelle erwähnt, dann keine korrelativen Derivationsbeziehungen angesetzt werden, wenn auf der Ebene der Signifikate nicht von einer Inklusions-, sondern von einer Intersektionsrelation auszugehen ist. Bei allen anderen Relationstypen handelt es sich nicht um Derivationsbeziehungen.

Allgemein kann nun formuliert werden, daB die in der empirischen Untersuchung zu behandelnden echten Derivations- oder Wortbildungsbeziehungen hinsichtlich zweier Lexeme genau dann bestehen, wenn in bezug auf deren Stämme zwischen den Inhaltsseiten eine Inklusions- und zwischen den Ausdrucksseiten eine Inklusions- oder eine Intersektionsrelation vorliegt.

An dieser Stelle wird der kritische Leser der vorliegenden Arbeit vermutlich fragen, wie es denn in diesem Punkt um die Heuristik bestellt sei. Der Autor steht auf dem Standpunkt, daß es im Rahmen dieses Vorhabens nicht darum gehen kann, ein Instrumentarium zu erarbeiten, mit dessen $\mathrm{Hilfe}$ es möglich wäre, zu bestimmen, wo Inklusions- oder wo Intersektionsrelationen vorliegen. Was die Ausdrucksseite anbelangt, gibt es wohl keine größeren Schwierigkeiten; für die Inhaltsseite aber wäre eine semantische Metasprache vonnöten. Da eine solche jedoch bisher noch nicht in überzeugender Form existiert, gibt es vorerst keine andere Möglichkeit als die, stillschweigend davon suszugehen, daß bei gemeinsamer Wurzel in der Regel auch ein gemeinsamer Inhalt vorliegt und daß sowohl der Autor wie auch der Leser dieser Arbeit zu denselben Schlüssen gelangen, zumal ja nicht unbedingt gesagt werden muB, welcher von zwei Inhalten jeweils im anderen enthalten sein soll. Nur dann, wenn trotz gemeinsamer Wurzel kein gemeinsamer Inholt anzusetzen ist, wird dies im einzelnen, sofern vorkomend, begründet.

Dennoch kann diese Situation nicht befriedigen, auch wenn andere (vgl.u.) auf dieser labilen Grundlage sogar die Derivationsrichtung best immen. 


\subsubsection{Suppletivismus}

Die soeben getroffenen festlegungen bezüglich der Inhalts- und der Ausdrucksseiten solcher Wortformen, die als in einer Derivationsbeziehung befindlich gedacht werden sollen, stellen uns vor das Problem, angeben zu müssen, welche Einheiten man noch als formal (und inhaltich) miteinander korrelierend auffassen will, und welche nicht mehr. Dieses frage, die oben ( 5 . 18, Anm. 18) bereits kurz angeschnitten wurde, soll nun geklärt werden. 46

Die Bestimmung der formalen und der inhaltlichen Nähe zweier Einheiten bzw. die der erlaubten Abweichungen von der jeweils als Bezugsform aufgefabten Gröbe soll sich von der Überlegung leiten lassen, daB jede der genannten Einheiten eine bestimmte Phonemkonfiguration mit einer bestimmten Konfiguration von Inhaltselementen darstellt. Bei dem Versuch, die Grenzen einer zulässigen Varianz festzulegen, geht man bisweilen davon aus, daß weniger der absolute Grad der beobachtbaren Divergenzen aussagekräftig sei als vielmehr die Antwort auf die Frage, ob die Unterschiede auf der Ausdrucks- und der Inhaltsseite "systemisch, sich regulär wiederholend sowie serienbildend sind" (KUBRJAKOVA 1974, 80). Hier wird implizit u.a. der Standpunkt vertreten, daB die formale Ahnlichkeit zweier miteinander verglichener Einheiten nicht durch solche Veränderungen in deren segmentphonematischem Bau aufgehoben wird, die innerhalb der gegebenen Sprache mehr oder weniger regulären Charakter aufweisen, sondern nur von solchen, die allgemein als 'Suppletivbildungen' bezeichnet werden. Eine wichtige Voraussetzung dafür, diese Auffassung auch in der vorliegenden Arbeit zu übernehmen, kann als erfüllt betrachtet werden, da MEL'CuK (1972) eine prinzipielle Eingrenzung dessen gelungen ist,

46 An dieser Stelle sei angemerkt, da auch andere Standpunkte verteten werden. So gehen bspw. ZENKOV $(1969,61)$ oder OLIVERIUS $(1976,35)$ davon aus, daß bei inhaltlicher Identität zweier miteinander verglichener Einheiten die phonematische Ähnlichkeit überhaupt keine Rolle spiele. Eine solche Auffassung würde implizieren, daß man sich Lexeme wie XpaбрыЙ und Склость theoretisch als in einer Ableitungsbeziehung stehend vorstellen könnte, da die in ihnen enthaltenen Wurzeln храбр- und वмeлinhaltlich identisch und somit nach obigem Verständnis als Varianten einer und derselben Wurzel zu begreifen sind. 
worum es sich bei Suppletivformen handelt. Die wesentlichen Punkte des genannten Verfahrens sollen hier stark verkürzt wiedergegeben werden.

Nach MEL'CuK gilt der formale Unterschied $F^{\prime}$ zwischen zwei Wortformen $A$ und $B$ für eine Sprache $S$ dann als 'normal', wenn $A$ und 8 sich ausschlieBlich hinsichtlich der in ihnen repräsentierten $A f f i x e$ und/oder Flexionsendungen unterscheiden ( $2 . B$. (A) dom (Haus) - (B) domik (Häuschen) oder (A) doma (des Hauses) - (B) domiku (dem Häuschen)) oder wenn $F^{\circ}$ durch solche Veränderungen im segmentphonematischen Bau der nicht als Bezugsform aufgefaßten Wortform bedingt ist, die für $S$ normal sind (Bsp.: $[1, u b,-i-t$,$] (1ieben)-[1,ubl,-ij] (ich liebe)).$

Der inhaltliche Unterschied $I^{\circ} z$ wischen $A$ und $B$ heibt dann normal, wenn er innerhalb 5 mittels Affixen susgedrückt werden kann, wobei als bekannt vorausgesetzt wird, was in $S$ affixale und was nichtaffixale Bedeutungen sind (Bsp.: dom (Haus) domiśče (großes Haus)).

'Normalität'ist insofern eine variable Gröbe, als nicht nur gesagt werden kann, etwas sei normal oder nichtnormal, sondern auch, etwos sei mehr oder weniger normal bzw. nichtnormal. Eine klare Grenze zwischen eben noch normalen sowie schon eher nichtnormalen inhaltlichen und formalen Unterschieden zieht MEL'Cuk nicht, doch kann man seiner Uberlegung folgen, dab eine in Vergleich mit einer Bezugsform auftretende Veränderung $F^{\prime}$ im segmentphonematischen Bau einer Wortform um 80 normaler ist, je häufiger sie in $S$ beobachtet werden kann. Für $I^{\circ} g i l t$, daB ein affixaler Inhalt $I_{l}$, der in hunderten von Wortparen vorkommt, normaler ist als ein Inhalt 12 , der sich lediglich für einige wenige Paare belegen läbt.

Aufgrund dieser Prämissen erweist sich der Suppletivismus als eine Relation, die quantitativ erfaBt werden kann. Nach MEL'Čuk liegt zwischen zwei Wortformen $A$ und $B$ echter Suppletivismus dann vor, wenn im Vergleich mit der Bezugseinheit $I^{\prime}$ maximsl normal sowie $F^{\prime}$ maximal nichtnormal ist, wobei sich $A$ und $B$ hinsichtlich $1^{\circ}$ in komplementärer Distribution befinden. 47 Ein Bei-

47 Einheiten gelten dann als komplementär distribuiert, wenn sie niemals in einem und demselben Kontext begegnen. 
spiel: Obwohl zwischen ihnen I'maximal normal und $F^{\prime}$ minimal normal sind, gelten im Russischen die Wörter jazykovedenie (Sprachwissenschaft) und lingvist (Linguist) als nichtsuppletiv, da es neben jazykovedenie das Wort jazykoved (Sprachwisssenschaftler) gibt sowie neben linguist auch das Wort linguistika (Linguistik). Aus diesem Grunde befinden sich die Wurzeln jazyk - und lingv- nicht in einer komplementären Distribution hinsichtlich des inhaltlichen Unterschiedes 'Wissenschaft über ...' - 'Fachmann auf dem Gebiet der Wissenschaft über ...'. Anders verhält es sich beispielsweise bei den Wörtern stirat' (waschen) und pracka (Wäscherin): stir-und praćbefinden sich - grob gesagt - unter anderem hinsichtlich der Inhalte 'Handlung' und 'Träger der Handlung' in komplementärer Distribution, weshalb diese Lexeme als Suppletivformen aufzufassen sind.

Auch in der vorliegenden Arbeit wird keine eindeutige Grenze zwischen 'Normalität' und 'Nichtnormalität' in dem Sinne gezogen, dab etwa folgendes gesagt würde: Unterscheiden sich zwei miteinander verglichene Einheiten $A$ und $B$ in ihrem segmentphonematischen Bau dadurch, daB eine von ihnen durch mehr als fünfig Prozent der in ihr entholtenen Phoneme von der BezugsgröBe differiert, so handelt es sich um suppletive Bildungen. 48 Ein derartiges Vorgehen kann sich deshalb als inadäquat erweisen, weil im Vergleich mit der Bezugaeinheit durchaus solche Veränderungen in segmentphonematischen Bau einer Wortform denkbar sind, die mehr als fünfzig Prozent ihrer Phoneme berühren und dennoch ols mehr oder weniger normal gelten können.

Für die Zwecke dieser Arbeit ist es ausreichend, mit dem oben formulierten Begriff der Normalität von $F^{\circ}$ oder von $I^{\circ}$ zu operieren und suppletive Bildungen in der Regel nur dort anzusetzen, wo für zwei Einheiten $A$ und $B$ folgendes gilt: $I^{\circ}$ kann $z$ wischen ihnen als normal oufgefabt werden, ferner liegt in bezug auf diese Größe komplementäre Distribution von $A$ und $B$ vor, und schlieblich ist $F^{\circ}$ unikal in dem Sinne, dab es sich

48 Einen solchen Vorschlag machen LOPAIIN Und ULUCHANOV (1972, 205f). 
hier um eine solche Veränderung im segmentphonematischen Bau einer der beiden Einheiten handelt, die in S die Systemfrequenz 1 besitzt. - Mit anderen Worten: Als suppletiv sollen hier definitionsgemäB nur solche Wortformen verstanden werden, bei denen der formale Unterschied zur Bezugsform innerhalb von $S$ absolut nichtregulär (singulär) ist. Als mehr oder weniger regu-

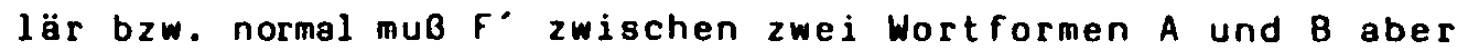
schon dann aufgefalt werden, wenn es auch nur ein einziges Paar $C$ und $D$ gibt, zwischen dessen Gliedern sich die gleiche segmentphonematische Veränderung ansetzen läßt wie bei $A$ und $B$.

\subsubsection{Derivationsrichtung}

Wie bereits im vorangegangenen Kapitel deutlich wurde, benötigt man, um je zwei zueinander in irgendeiner Beziehung stehende Wortformen oder Lexeme inhaltlich und formal miteinander vergleichen zu können, eine(s) von ihnen als Ausgangsgröße, in bezug auf die man jeweils Aussagen über eine Gleich-oder Verschiedenartigkeit hinsichtlich der genannten Bereiche treffen kann. Diese Rolle der BezugsgröBe soll im Rahmen der vorliegenden Arbeit das sogenannte abgeleitete Wort spielen.

Eine im Zusammenhang mit der Wortbildung als linguistischer Disziplin wesentliche und unterschiedlich beantwortete frage ist demnach die, von welchen Kriterien man sich leiten lassen soll, wenn es festzustellen gilt, welches von zwei in einer $A b-$ leitungsbeziehung stehenden Wörtern das obgeleitete und welches das ableitende ist.

Nicht näher berücksichtigt wird in der vorliegenden Arbeit der Ansatz des vor allem von S.K. ŠAUMJAN und P.A. SOBOLEVA entwickelten 'applikativen generativen Modells', mittels dessen logische ('genotypische') Aussagen angestrebt werden, die der 'phänotypischen' Ebene des konkreten sprachlichen Materials vorgelagert sind und die Abbildung eines universalen semiotischen Systems leisten sollen (vgl. bspw. SAUMJAN 1965; SOBOLEVA 1972; ŚAUMJAN, SOBOLEVA 1963, 1968, 1972). Stark simplifiziert bedeutet dies in bezug auf die Wortbildung, daB ein 'Wortgenerator' sogenannte Relatoren $(R)$ auf sogenannte Semione ( 0$)$ appliziert. Affixe zur Bildung von Verben gelten dabei als Relatoren zum Verb und werden mit $R_{1}$ bezeichnet, solche zur Bildung von Substantiven mit $R_{2}$, adjektivische Affixe mit $R_{3}$ usw. Aufgrund der Prämisse, daß dann, wenn $X$ ein Lexem ist, es sich auch bei $R_{1}, 2, \ldots, n-1, n X$ um ein Lexem handelt, geht man daran, die sich in bestimmten 'Dérivationsschritten' manifestierende 'Ableitungsgeschichte' von Lexemen darzustellen. So kann beispielsweise die 'genoty- 
pische Reihe $R_{1} O \rightarrow R_{2} R_{1} O \rightarrow R_{3} R_{2} R_{1} O \rightarrow R_{2} R_{3} R_{2} R_{1} 0$ 'phänotypisch' folgendermaßen besetzt sein: proizvodit' (produzieren) + proizvodstvo (Produktion) $\rightarrow$ proizvodstvennyj (Produktions-) - proizvodstvennik (Produktionsarbeiter)(vgl. SAUMJAN, SOBOLEVA 1972, 347). Da man durchaus die Auffassung vertreten kann, proizvodstvennik sei nicht von proizvodstvennyj abgeleitet, sondern von einer Wortverbindung wie proizvodstvennyj rabocij (Produktionsarbeiter), wird folgender genereller Mangel dieses Modells deutlich: Es fehlen nähere Angaben dazu, nach welchen Kriterien man zu dem ableitenden sowie zu dem entsprechenden abgeleiteten Wort gelangt und wie es möglich ist, die jeweilige Abfolge der Wörter innerhalb der Kette widerspruchsfrei zu bestimmen. Mithin kann, zumindest bezüglich der Wortbildung, nur mit Abstrichen davon die Rede sein, daB die SAUMJANschen Postulate der Beschreibungs- oder gar der Erklärungsadäquatheit erfüllt sind. Dieses Vorgehen ist daher vorläufig nicht intersubjektiv nachprüfbar, weshalb die anstehenden Probleme mittels des generativen Prinzips noch keiner befriedigenden Lösung zugeführt werden können.

Welche Gesichtspunkte berücksichtigen beispielsweise die Autoren des Abschnittes 'Wortbildung' in den Standardwerken AG-70 und $A G-80$ ? LOPATIN und ULUCHANOV legen fest, daB das von ihnen so genonnte motivierte Wort über die folgenden Merkmale verfügt (vgl. AG-70, 38):

1) Für den Fall, daB die in einer Derivationsbeziehung stehenden Wörter sich (unter anderem) hinsichtlich ihrer nichtgrammatischen Bedeutungen unterscheiden, gilt dasjenige als abgeleitet, dessen Stamm sich durch die gröBere formale Komplexität auszeichnet. Als Beispiel wird das Paar goroch (Erbse (als Pflanze)) - gorosina ((einzelne) Erbse) angeführt.

In der $A G-80(I, 133)$ wird näher erläutert, was man sich unter einem formal komplexeren Stam vorzustellen hat. Als formal komplexer gilt der Stamm, innerhalb dessen sich die größere Anzahl von Lautsegmenten ausgliedern läbt; dies unabhängig davon, ob die Segmente über eine Bedeutung verfügen oder nicht. - An dieser Stelle sei zum wiederholten Male angemerkt, daß es schwierig sein dürfte, letzteres zu begründen, da es wohl kaum Lautsegmente gibt, die sich nicht durch irgendeine 'Bedeutung' (und sei es durch eine bestimmte Signalfunktion) auszeichnen.

2) Differieren die in einer Derivationsbeziehung stehenden Wörter in bezug auf ihre nichtgrammatischen Bedeutungen, und ist keines von ihnen formal komplexer als das andere, so ist dasjenige Wort als das abgeleitete anzusetzen, das sich durch seine gröBere inhaltliche Komplexität auszeichnet. ${ }^{49}$ Beispiel: $[x, i m, i j-a]$ (Chemie) - [x,im,ik-ø] (Chemiker; jemand, der die

49 Der Begriff der inhaltlichen Komplexität wird weder in der AG-70, noch in der AG-80 näher bestimmt; ein Umstand, der es im Grunde von vornherein unmöglich macht, mit diesem Kriterium widerspruchsfrei zu operieren. 
Wissenschaft der Chemie betreibt). 50

3) Sind bei zwei in einer Derivationsbeziehung stehenden Wörtern alle Inhaltselemente bis auf die grammatischen identisch, dann gilt (a) bei einem Paar 'Adjektiv - Substantiv (das dasselbe Merkmal bezeichnet wie das Adjektiv; Bsp.: krasnyj (rot) - krasnota (Röte))' das Substantiv als abgeleitet, weil es sich bei der 'Merkmalsbedeutung' um die 'allgemeine kategoriale Bedeutung' des Adjektivs und nicht um die des Substantivs handele.

Irifft die 'Gleichartigkeit' bezüglich der nichtgrammatischen Inhaltselemente (b) auf ein Paar 'Adjektiv - Adverb' $z u$, wird wieder das formale Kriterium (1) bemüht. Bsp.: smelyj (kühn) (Adj.) - smelo (kühn) (Adv.).

In der $A G-80(I, 133)$ werden den sich durch das Merkmal 'Gleichartigkeit der nichtgrammatischen Inhaltselemente' auszeichnenden Gruppen auch Paare vom Typ 'Verb - Substantiv (das dieselbe Handlung benennt wie das Verb; Bsp.: vychodit' (hinausgehen) - vychod (Hinausgehen))' zugeordnet. Hier gilt (wie in (a)) das Substantiv als abgeleitet, da die 'Handlungsbedeutung' die 'allgemeine kategoriale Bedeutung' des Verbs und nicht die des Substantivs sei.

Nach dem in dieser Arbeit verfolgten Prinzip werden zwischen den zu (3) zählenden Wörtern sämtlich nichtkorrelative Derivationsbeziehungen des Typs (5) (vgl. S. 42) angesetzt, die aus der nachfolgenden empirischen Untersuchung herausfallen, da sich der tatsächlich vorhandene inhaltliche Unterschied ausschließlich in den grammatischen Inhaltselementen

SO Dieses auch in der AG-80 ( 1,133$)$ angeführte 8eispiel ist natürlich überaus unglücklich gewählt, da es Bildungen nach demselben Muster ('Wissenschaft - der jenige, welcher diese Wissenschaft betreibt') gibt, bei denen infolge (1) die Derivationsrichtung genau umgekehrt anzusetzen wäre. Vgl. etwa $[b, i o ́ l o g-a]-[b, i o l o g,-i j-a]$. E in solches Vorgehen führt also zumindest in diesem $\mathrm{Fall}$ zu nicht wünschenswerten Inkongruenzen bei der Beschreibung (vgl, auch CHOCHLACEVA 1973, 103). Ein anderes, in der AG-80 ( $I, 133$ ) genanntes Beispiel, näml ich худоnнHK художница (мениина-худажник) 'paßt' hier besser. - Um Mißverständnissen vorzubeugen sei festgehalten, daß es sich in diesem Fall nicht um eine solche Beziehung handelt, die der Gruppe (8) (vgl. 5. 42) zuzuordnen wäre, da bei художниК - Худопница auf der Inhaltsseite keine Intersektion vorliegt: Худопницl ist zwar darstellbar als 'худалнци + weibl. Geschlecht', Худопния aber nicht als 'худоп̆ни + männl. Geschlecht' . Dies wird deutlich in Texten wie: 'Oна заслуленная худояник Co10за'. Aus diesem Grund liegt hier formal wie auch inhaltlich eine Inklusions- und damit eine echte Wortbildungsbeziehung vor. 
manifestiert. Richtig ist natürlich, daß in vielen Fällen auch bestimmte Ausdruckselemente des Stamms (bspw. Affixe) die Hortartenzugehörigkeit des entsprechenden Lexems prädizieren, doch da es in diesem Zusammenhang zumindest schwierig ist, von Inhalten zu reden, kann bedingt eine Gleichartigkeit der nichtgrammatischen Inhaltselemente angenommen werden. Einen ähnlichen Standpunkt vertritt z.B. LICHIMAN (1973, 139), indem sie davon ausgeht, daß es sich etwa bei chorosij (gut; Adj.) - choroso (gut; Adv.) um parallel gebildete Wörter handelt, bezüglich derer niemals gesagt werden kann, das eine sei vom jeweils anderen abgeleitet. Auch LOPATIN Und ULUCHANOV sind in einer früheren Arbeit der Ansicht, daß "man in einer Reihe von Fällen nur von einer Wortbildungskorrelativität der Wörter reden kann, da systemische Faktoren fehlen, die auf Motivationsbeziehungen hindeuten ('gegenseitige Motivation')" (in SVEDOVA 1966, 58f). Als Beispiele werden Wortpaare wie degradirovat' (degradieren) - degradacija (Degradierung), opportunizm (Opportunismus) - opportunist (Opportunist), tolknut' (stoßen; vollendeter Aspekt) - tolkat' (stoBen; unvollendeter Aspekt) genannt (ebd., 59).

Ebenfalls in der $A G-80(I, 133)$ wird schlieBlich noch ein stilistisches Kriterium eingefüht:

4) Von einem Wort, das stilistisch nicht neutral ist, kann kein anderes, stilistisch neutrales Wort abgeleitet sein. Bildungen des Typs qumanitar (humanistisch Gebildeter) oder korabel (Schiffbauer) gelten somit als von den Adjektiven qumanitarnyj (humanistisch) bzw. korabel'nyj (Schiffs-) abgeleitet; dies ungeachtet dessen, dab nach (1) die andere Richtung angesetzt werden müBte.

Aus dieser Ubersicht geht klar hervor, daß dann, wenn sie nicht zu ganz willkürlichen Entscheidungen greifen (vgl. (3) und (4)), LOPATIN und ULUCHANOV dem formalen Kriterium den ersten Rang bei der Bestimmung der Derivationsrichtung zuweisen. Inholtliche Aspekte berücksichtigen sie erst dann, wenn das formale Prinzip nicht anwendbar ist (genauso verfährt auch BYKOVA (1974, 45)). Die Bevorzugung der formalen Seite kann, wie gezeigt, zu unerwünschten Inkongruenzen bei der Deskription führen, da nicht auszuschlieBen ist, dab die auf diese Weise ermittelten Ergebnisse in klarem Widerspruch zu den mittels des inhaltlichen Kriteriums erzielten Resultaten stehen. Der Hauptgrund dafür, dab man bei der Bestimmung der Derivationsrichtung nicht primär mit formalen Gesichtspunkten operieren sollte, ist also der, daB ein solches Verfahren niemals in allen Fällen je für sich allein angewendet werden kann, so dab auch andere Kriterien zu Rate gezogen werden müssen. Vergegen- 
wärtigt man sich zudem, daB Wörter in der Regel ausschlieblich zu dem Zweck gebildet werden, neue Referenten in ganz bestimmter Weise zu benennen, so erscheint es angebrachter, dann, wenn überhaupt einem der beiden in der AG-70 sowie in der AG-80 zugrunde gelegten Kriterien der Vorzug gegeben werden soll, den inhaltlichen Aspekt stärker zu gewichten.

Zu den wenigen Linguisten, die die Auffassung vertreten, die Derivationsrichtung lasse sich allein dadurch feststellen, daB man die in einer Ableitungsbeziehung stehenden Wörter jeweils inhaltlich analysiert, zählt LICHTMAN. 51 Einen frühen Verteter findet dieser standpunkt in VINOKUR, der zu Recht annimmt, dab bei der Wortbildung in erster Linie eine bestimmte inhaltiche Verschiebung vom ableitenden zum abgeleiteten Wort stattfindet. Für ihn unterscheidet sich nämlich der sogenannte abgeleitete Stamm vom nichtabgeleiteten vor allem "durch einen anderen Bezug zum Gegenstand der Wirklichkeit, zu seinem Bezeichneten. [...] Ein Wort kann einen bestimmten Gegenstand der Wirklichkeit entweder unmittelbar benennen, oder indem es diese oder jene Relation zwischen dem gegebenen Gegenstand der Wirklichkeit und anderen herstellt" (VINOKUR 1946, 316). Aus diesem Grund geht es VINOKUR weniger um die feststellung dessen, $i$ i e ein Wort abgeleitet ist, sondern eher darum, wo$v$ o $n$ os als abgeleitet gedacht werden kann. 52 Dabei findet die Deriviertheit der entsprechenden Wörter ihren Ausdruck "in den Beziehungen zwischen den in der Struktur dieser Art von Wörtern zutage tretenden bedeutungshaltigen Lautkomplexen" (ebd., 317), wobei sich die Bedeutung von Wörtern "mit abgeleitetem Stamm immer mittels eines Verweises auf die Bedeutung des

51 "Производность - одна. И если слово производно "По смислу", значит оно воОбще производно, т.е. гроизводно и "фориально", хотя [...] оно молет бытть формально менее слопно. Иными словами, для производного слова обязательна лишь бо́льшая семантическая слонность по сравненио с производяцвм, но не формальная" $(1973,138)$. Die möglichen Divergenzen hinsichtlich der inhaltlichen und der formalen Derivation von Wörtern behandeln z.B. auch ERMAKOVA (1972) und MEL'ĆUK (vgl.u.).

S2 Vgl. auch ZVEREV $(1972,72)$, der der Meinung ist, es sei sinnvoller, bei der Bestimmung der Derivationsrichtung statt vom ableitenden vom abgeleiteten Wort auszugehen, da jedes "Мотивированное всегда "ищет" свое мотивиругацее." 
entsprechenden primären Stamms bestimmen läBt" (ebd.). 53

Auch MEL'ĆUK (1967; 1969) beschäftigt sich mit der Frage, welches von zwei in einer Ableitungsbeziehung stehenden Wörtern mittels des jeweils anderen erklärt werden könne. Bei der Analyse der größeren/geringeren inhaltlichen Komplexität solcher Wörter nennt er eine Reihe von fällen, in denen er zu Lösungen gelangt, die offensichtlich nicht so ohne weiteres nachvollzogen werden können. So setzt er für das Wortpar sírokij (breit) - śirina (Breite) die Derivationsrichtung vom Substantiv zum Adjektiv an, da er letzteres für inhaltich komplexer hält (breit = von grober Breite; vgl. 1967, 357), wohingegen $2 . B$. in der $A G-70$ (allerdings aufgrund einer apriorischen Festlegung) gesagt wird, daB es sich beim Substantiv um das abgeleitete Lexem handele (97). Daher ist die folgende Behauptung MEL'ČUKs $(1969,135)$ auf den ersten Blick nicht wenig verwunderlich, daB "die größere/geringere inhaltliche Komplexität von dem Sinne nach miteinander in einer Beziehung stehenden Wörtern deren objektive Eigenschaft sei, die so oder anders in den möglichen Verwendungen dieser Wörter usf. zum Vorschein kumme. Das semantisch einfachere von zwei Wörtern tritt in Laufe der Untersuchung zutage und wird nicht der Laune des forschers nach ausgewählt" (diese Auffassung vertritt auch KUBRJAKOVA (in SEREBRENNIKOV 1972, 374). Doch hat MEL'ĊUK im Ansatz gezeigt, daB man bei konsequenter und vor allem bei kohärenter Analyse zu befriedigenden Ergebnissen gelangen kann.

Vorläufig bleibt es jedoch dabei, dab ein "rein semantisches Kriterium bei der Feststellung von Motivationsbeziehungen nicht isoliert verwendet werden kann, da ein und dieselben semantischen Relationen strukturell unterschiedlich realisiert sein können. So wird beispielsweise die Bedeutung 'Werden eines Merkmals' bei Verben häufig durch die Bedeutung des Merkmals

53 Vgl. etwa auch TICHONOV $(1967,112)$ : "Семантика производящей основы является тем фундаментом, на котором "воздвигается" семантика производнОЙ ОСНОы" oder KRYLOV $(1963,34)$, für den die nichtgrammatischen Bedeutungen abgeleiteter Wörter enger sind als die der ableitenden, was es gestattet, "определить их значение посредством ссылюи на значение производящей основы, т.К. производная основа вырапает частное понятие, а производяшая - балее ощие." 
expliziert (belyj (weiB) - belet' (weiB werden)), was jedoch die Möglichkeit nicht ausschliebt, ein Merkmal als Resultat des Werdens darzustellen (zret' (reif werden) - zrelyj (reif))" (LOPATIN, ULUCHANOV in ŚVEDOVA 1966, 58). Solche und ähnliche Beispiele könnte man zuhauf anführen, und solange es nicht gelingt, bspw. ein erklärendes Wörterbuch zu erstellen, in dem (bis auf solche Lexeme, die als nichtabgeleitet gelten können) jedes Wort unter Verwendung desjenigen inhaltlich weniger komplexen oder weniger speziellen Wortes definiert wird, mit dem es als in einer Ableitungsbeziehung befindlich gedacht werden kann, ist es kaum möglich, widerspruchsfrei und intersubjektiv nachvollziehbar mit dem inhaltlichen Kriterium zu arbeiten.

Wir müssen also konstatieren, dab es zwar prinzipiell möglich ist, die Derivationsrichtung zwischen zwei in einer Ableitungsbeziehung stehenden Wörtern mittels des inhaltlichen Kriteriums zu bestimmen, es zur Zeit jedoch an geeigneten Hilfsmitteln mangelt, die es gestatten würden, ein solches Vorgehen zu objektivieren. Das formale Kriterium vermag das Geforderte ebenfalls nicht zu leisten, da die in einer Derivationsrelation stehenden Wörter oft stämme von gleichartiger phonematischer Komplexität aufweisen. Da auch die gleichzeitige Berücksichtigung des inhaltichen wie des formalen Kriteriums, wie gezeigt, zu Inkongruenzen bei der Beschreibung führen kann und es sich leider ganz und gar nicht so verhält, dab die "Formel 'A ist abgeleitet von $B^{\prime}$ als Vorhandensein eines bestimmten Typs durchsichtiger, leicht identifizierbarer Relationen zwischen $A$ und B dechiffriert werden kann" (KUBRJAKOVA in SEREBRENNIKOV 1972, 380), ist man gezwungen, zu einem anderen Prinzip zu greifen; dies in der Hoffnung, daB die mit ihm zu erzielenden Resultate weniger widersprüchlich oder zufällig sein werden.

Die Methode, mit der sich nach Lage der Dinge wohl am erfolgversprechendsten operieren läBt, nannten LOPATIN und ULUCHANOV zwar schon im Jahre 1966 (noch früher findet sich dieser Ansatz auch bei MARCHAND (1974, 246)), doch in die Praxis wurde sie von ihnen nie umgesetzt: "Die Motiviertheitsbeziehung (die Motivationsrichtung) wird auf der Grundlage analoger Bildungen festgestellt, die im synchronen Sprachsystem existieren und auch mit 
Hilfe einer Bestimmung der quantitativen Korrelation gleichwurzliger Wörter: beispielsweise sind Substantive des Typs chod'ba (Gehen) erheblich seltener als Verben des Typs chodit' (gehen), und Substantive des Typs sus' (Trockenes), sin' (Bläue) sind seltener als Adjektive des Typs suchoj (trocken), sinij (blau), und deshalb müssen diese Substantive als sekundär bzw. als motiviert angesehen werden" (in ŠVEDOVA 1966, $57) .54$

Die Merkmale der gröBeren/geringeren formalen/inhaltlichen Komplexität galten den beiden Autoren zu der Zeit noch als sekundär. "Am häufigsten zeigen sich diese allgemeinen Kriterien für eine Motiviertheit in der komplexen formalen Struktur und in der dieser entsprechenden semantischen Struktur" (ebd., 58). 55

54 Die Frage ist hier natürlich, welche Art von Häufigkeit LOPAIIN und ULUCHANOV gemeint haben können, die systemische oder die pragmatische. Für die Systemfrequenz spricht, daß die Autoren jeweils von Substantiv-, von Adjektiv- und von Verbtypen reden. Unklar bleibt zwar indes, was sie mit dem Terminus 'Typ' meinen, doch sind in diesem Fall darunter wohl Substantive mit dem Suffix -6 - bzw. mit Nullsuffix, nichtaffigierte (prä- und suffixlose) Adjektive und Verben mit dem Suffix - $h$ - zu verstehen. Anzunehmen ist daher, daß das Verfahren darauf hinausläuft, aus einem wörterbuch für alle Typen, die man im Russischen ansetzen kann, deren Vertreter zunächst zu exzerpieren und dann zu addieren, um so etwa zu Aussagen wie den folgenden zu gelangen: 'Nichtaffigierte Adjektive sind systemisch häufiger als Substantive mit Nullsuffix und daher als primär anzusehen' oder 'Substantive mit dem Suffix - $6-$ sind systemisch weniger häufig als Verben mit dem Suffix - $h$ - und haben daher als sekundär zu gelten'. Dies ist zwar denkbar, doch unter anderem deshalb wenig wahrscheinlich, weil ja dann die einleitende Bemerkung der Autoren keinen Sinn hätte. Es sollen nämlich die quantitativen Korrelationen qleichwurzliger Hörter bestimmt werden, und dies ist mit einer systemischen Frequenzbetrachtung nicht möglich, da die zu vergleichenden Wörter immer die Systemfrequenz 1 besitzen. Doch kann man sich unter Umständen auch Aussagen wie diese vorstellen: 'Zu dem nichtaffigierten Adjektiv сухой gibt es ein gleichwurzliges Substantiv mit Nullsuffix Cywb, und da Bildungen dieses Typs systemisch weniger frequent sind als die des erstgenannten Typs, sind sie als abgeleitet aufzufassen.' Denkt man konsequent weiter, so ergibt sich folgendes Bild: Adjektive beispielsweise mit dem Suffix $-\mathrm{H}$ - sind systemisch häufiger als nichtaffigierte (die in der Literatur durchweg als nichtabgeleitet bezeichnet werden). Finden sich nun in beiden Mengen Adjektive mit je gleicher Wurzel, so sind die nichtaffigierten als abgeleitet zu betrachten usf. - Der skizzierte Sachverhalt legt somit den SchluB nahe, daB LOPATIN und ULUCHANOV die pragmatische Frequenz der miteinander zu konfrontierenden gleichwurzligen Wörter gemeint haben müssen.

55 Daß dieser hinsichtlich der affixalen Wortbildung u.a. auch von ZEMSKAJA 
Diesem Kriterium liegt vermutlich die Überlegung zugrunde, dab es sich bei der Wortbildung um die "Kombination vorhandener Wörter oder Stämme miteinander oder mit besonderen - frei im Satz nicht vorkommenden - Bildungselementen" (FLEISCHER

${ }^{3} 1974$, 9; Unterstreichung von mir - J.k.) handele. Wenn also die Notwendigkeit besteht, neue Referenten zu denotieren, die sich durch bestimmte inhaltliche Korrelationen mit solchen Referenten auszeichnen, für die in der betreffenden Sprache bereits bestimmte Zeichen vorhanden sind, können letztere in bestimmter Weise modifiziert werden, um die erforderliche Denomination zu ermöglichen. Für unsere Zwecke soll nun davon ausgegangen werden, daB die primären Zeichen einer gegebenen Sprache pragmatisch frequenter sind als die sekundären und es sich bei ihnen aufgrund dieser Beziehung um die ableitenden handelt (die Termini 'primär' und 'sekundär' sind nicht im Sinne eines Nacheinander zu interpretieren, da beide Arten von Zeichen im Sprachsystem synchron neboneinander existieren).

Für die in dieser Arbeit anzustollende empirische Untersuchung wird folgendes festgelegt: Ist von zwei Lexemen, die untereinander in einer Derivationsbeziehung stehen, eines (nennen wir es $X$ ) pragmatisch häufiger als das andere (wir wollen es mit $x^{\prime}$ bezeichnen), gilt also $f(x)>f\left(x^{\circ}\right)$, dann sei (in der Regel, Ausnahmen vgl.u.) $x$ das ableitende Wort oder das 'Derivatem' und $x^{\prime}$ das abgeleitete Wort oder das 'Derivat'.

Das bisher umfangreichste Häufigkeitswörterbuch des Russischen (ZASORINA 1977) kann als durchaus akzeptable Basis für die Bestimmung der Derivationsrichtung aufgrund der quantitativen Beziehungen zwischen zwei in einer Ableitungsbeziehung stehenden Wörtern gelten. Die Materialgrundlage dieses Wörterbuchs bilden eine Million konkrete Wortformen, die vor ihrer Eingabe in den Computer mittels bestimmter Regeln (vgl. ZASORINA $1977,13 \mathrm{ff}$ ) auf die so genannten Alisgangswortformen (die Lexeme) zurückgeführt wurden. Von den konkreten Wortformen entfallen je ca. 250000 (a) auf Texte der künstlerischen Pross, (b) auf solche der modernen realistischen Dramaturgie, (c) auf wissenschaftlich-publizistische und (d) auf Zeitungs- bzw. Zeitschriftentexte. Das Wörterbuch enthält eine alphabetische Wortliste, in der alle unterschiedlichen Lexeme (insgesamt etwa 40000 ) unabhängig von der Häufigkeit der sie repräsentierendenkonkreten Wort formen umfaBt sowie eine zweite Wortliste, in

(55) $(1966,4)$ als der Allgemeinfall bezeichnete Sachverhalt tatsächlich nur für Spezialfälle zutrifft, versteht sich aufgrund des weiter oben Gesagten von selbst. 
die lediglich solche Lexeme aufgenomen sind, deren konkrete Wortformen mit einer Häufigkeit von 10 und mehr begegneten.

Obwohl natürlich evident ist, daB 40000 Wörter lediglich einen Ausschnitt aus der Lexik der russischen Sprache verkörpern, kann dennoch, wie der empirische Teil der vorliegenden Arbeit zeigen wird, ein grober Teil des z.B. in der AG-70 und in der AG-80 repräsentierten Materials quantitativ erfaBt werden. Selbstverständlich ist es prinzipiell nicht wünschenswert, daB ein Teil der in den betreffenden Grammatiken enthaltenen Lexeme nicht berücksichtigt werden kann, doch wiegt dieser Nachteil dank der objektiven Uberprüfbarkeit der mittels der quantitativen Methode erzielten Ergebnisse sowie auch wegen der geringen Vorkommenshäufigkeit der betreffenden Lexeme in den Texten des Russischen weniger schwer.

Die Angaben des hier benutzten Frequenzwörterbuches beziehen sich, wie bereits angedeutet, auf die pragmatische Häufigkeit konkreter Wortformen, die nach bestimmten Regeln zu Lexemen vereinigt werden. Der empirische Teil dieser Arbeit stützt sich also, was die quantitativen Angaben anbelangt, auf eine pragmatische Stichprobe. Als 'primär' sollen vorläufig alle Lexeme gelten, die der Klasse 'Adjektiv' angehören. Jedes dieser Lexeme wird mit der pragmatischen Häufigkeit seiner konkreten Wortformen versehen. Als 'sekundär' werden vorläufig alle Lexeme aufgefaBt, die mit den 'primären' aufgrund der oben getroffenen Festlegungen als in einer Derivationsbeziehung stehend gedacht werden können. Auch den 'sekundären' Lexemen wird die pragmatische Häufigkeit ihrer Wortformen zugeordnet. Nun werden der Reihe nach alle Pare von 'primären' und 'sekundären' Lexemen untersucht, die sich voneinander nach einem ganz bestimmten 'Muster' (hinsichtlich einer ganz bestimmten, rekurrenten konfiguration von Inhalts- und Ausdruckselementen) unterscheiden. Dabei bleibt die ursprüngliche Einteilung in 'primäre' und in 'sekundäre' Wörter dann bestehen, wenn in der Mehrzahl der Paare die Wortformen der in dem ersten Schritt vorläufig als iprimär' eingestuften lexeme pragmatisch häufiger sind als die der 'sekundären'. Ist dies nicht gegeben, wird die Derivationsrichtung 'umgedreht'.

Um eventuellen Unklarheiten vorzubeugen, sei das quantitative 
Prinzip zur Feststellung der Derivationsrichtung kurz exemplifiziert.

Aufgrund der oben getroffenen festlegungen lassen sich innerhalb der folgenden Lexempare jeweils Derivationsbeziehungen ansetzen:

1) bezobraznyj (empörend)

2) chiśçnyj (raubgierig)

- bezobraznik (Flegel)

3) ozornoj (ausgelassen)

$-$

4) staryj (betagt)

-

ozornik (Wildfang)

5) umnyj (klug)

(151) - umnik (kluger Mensch)

6) vinovnyj (schuldig)

$-$

vinovnik (Schuldiger)

7) vospitannyj (wohlerzogen)

vospitannik (Zögling)

Wir stellen fest, daB die Wortformen der Mehrzahl der a priori als 'primär' gesetzten Lexeme häufiger sind als die der 'sekundären', weshalb die angenommene Derivationsrichtung beibehalten werden kann. In den Paaren (2) und (6), bei denen unter alleiniger Berücksichtigung der quantitativen Verhältnisse (d.h. ohne eine gleichzeitige Bezugnahme auf die Verhältnisse in den anderen Paren) die Richtung vom Substantiv zum Adjektiv anzusetzen wäre, wird aus Gründen der Beschreibungshomogenität dennoch das Adjektiv als ableitend betrachtet, da bei den in Rede stehenden Substantiven auf der Ausdrucksseite unter anderem das Suffix - $\underline{i k-}$ und außerdem, genau wie in den übrigen Paaren, etwa das Inhaltselement 'einer, der sich durch das mit dem ableitenden Adjektiv benannte Merkmal auszeichnet' rekurrieren.

Anders verhält es sich dagegen bei den Paren
1) bolet' (krank sein)
- bol'noj (krank)
2) deżurit' (Dienst haben)
(8) -
dežurnyj (diensthabend)
3) nadoedat' (lästig werden)$$
-
$$$$
\text { nadoednyj (lästig) }
$$
4) pokorit'sja (sich fügen)
(2) - pokornyj (gefügig)
5) sklonit'sja (einwilligen) (19) - sklonnyj (geneigt)

Die Autoren der $A G-80(1,291)$ legen fest, daB in diesen Fällen die Adjektive mittels des Suffixes -n- von den entsprechenden Verben abgeleitet seien, und weisen ihnen (wohlgemerkt, den Adjektiven!) die 'Wortbildungsbedeutung' "Subjekt der (des) mit dem motivierenden Wort benannten Handlung (Zustandes)" zu. Wir 
dagegen wollen die Derivationsrichtung 'umdrehen', da nicht die von LOPATIN und ULUCHANOV als 'primär' gesetzten Verben, sondern die Adjektive aufgrund ihrer pragmatischen Häufigkeit als 'primär' aufzufassen sind, wobei die Paare (3) und (4) analog zum vorangegangenen Beispiel ebenso behandelt werden. Das in den Verben rekurrierende Inhaltselement könnte man umschreiben als 'das mit dem ableitenden Adjektiv benannte Merkmal an den Tag legen'. Da jedoch nicht bei allen angeführten Verben auch je ganz bestimmte Ausdruckselemte rekurrieren, sind die aufgezählten Pare im empirischen Teil dieser Arbeit bestimmten Untergruppen zuzuweisen.

Helches sind nun die Vor- und welches möglicherweise die Nachteile bei einer Bestimmung der Derivationsrichtung aufgrund von pragmatischen frequenzbetrachtungen?

Wie bereits angedeutet, wird von zwei in einer Derivationsbeziehung stehenden Wörtern das pragmatisch häufigere deshalb als das ableitende betrachtet, weil anzunehmen ist, die Sprecher einer Sprache bedienten sich (u.a.) bereits vorhandener Wörter oder Stämme, um mit ihnen und mit weiteren Mitteln bestimmte notwendige Denominationen vorzunehmen. Mit anderen Worten: Es ist bereits etwas vorhanden, auf das bei Bedarf 'zurückgegriffen' werden kann. Dies ist auch ein Grund dafür, zu sagen, von zwei in einer Derivationsbeziehung stehenden Wörtern sei das pragmatisch weniger häufige 'sekundär'.

Positiv zu vermerken ist vor allem, dab dieses Kriterium, im Gegensatz zu den üblicherweise angewendeten, für sich allein benutzt werden kann. Darüber hinaus läbt sich mit Hilfe dieser Vorgehensweise die Derivationsrichtung auf objektiver Grundlage ermitteln. Rein subjektive Unterschiede, wie sie (vorläufig noch) vor allem beim semantischen Kriterium von forscher zu Forscher auftreten können, lassen sich vermeiden; somit treten die weiter oben beschriebenen Inkongruenzen nicht in Erscheinung.

Die mit Hilfe des quantitativen Kriteriums erzielten Ergebnisse können natürlich nur in bezug auf einen synchronen 'Schnitt' gelten. Im vorliegenden fall deckt dieser Schnitt den Zeitraum ab, in dem die von den Verfassern des benutzten fre- 
quenzwörterbuches verarbeiteten Texte entstanden sind. Dies bringt natürlich den 'Nachteil' mit sich, daB im Lauf der Zeit in einer mehr oder weniger großen Anzahl von fällen deshalb eine andere Derivationsrichtung angesetzt werden müBte, weil man sich ja durchaus vorstellen kann, daB einmal gebildete Wörter nach und nach pragmatisch häufiger werden als die, von denen sie ursprünglich als abgeleitet gedacht werden konnten. Dies wiederum heiBt allerdings nicht, daB innerhalb ganzer Reihen von sich nach einem und demselben 'Muster' unterscheidenden Paaren die andere Richtung angesetzt werden müßte, da dies ja erst dann erfolgen soll, wenn die Mehrzahl der Pare von den veränderten Häufigkeitsverhältnissen betroffen sein wird.

Man könnte folglich unter Umständen einen Mangel darin sehen, daB die vorliegende Arbeit möglicherweise zu einem späteren Zeitpunkt in bestimmten Abschnitten des empirischen Teils umgeschrieben werden müBte. Dies kann aber auch ein Vorteil sein, da es auf diese Weise möglich ist, boi einem Vergleich der an verschiedenen synchronen 'Schnittlinien' ermittelten Ergebnisse auch diachrone Prozesse innerhalb des Russischen sichtbar zu machen.

\subsubsection{1. 'Wortbildungsbedeutung'}

Wir wollen uns nunmehr Fragen zuwenden, die im Zusammentiang mit der sogenannten Wortbildungsbedeutung stehen. Dabei ist bemerkenswert, daB dieser Begriff fast ausschlieblich von sowjetischen Linguisten verwendet wird und in einer Reihe von Aufsätzen sowie auch in einigen Monographien, wie z.B. In der von REVZINA (1969), vON MANUĆARJAN (1975) oder von ULUCHANOV (1977) abgehandelt worden ist.

Nun verhält es sich bei weitem nicht so, dab hinsichtlich der 'Wortbildungsbedeutung' eine einheitliche Position vertreten wird; vielmehr bestehen sowohl in bezug auf den Inhalt, wie auch auf den Ausdruck der in Rede stehenden GröBe unterschiedliche Auffassungen. Die verschiedenen Standpunkte sollen zunächst kurz vorgestelit werden, um daran die Erörterung der 
Frage anzuschlieben, ob es tatsächlich sinnvoll ist, die nichtgrammatischen Bedeutungen von Lexemen weiter in 'lexikalische' und 'derivationelle' zu unterteilen.

Uberaus häufig sind Versuche, die 'Wortbildungsbedeutung' durch eine Abgrenzung von der sogenannten lexikalischen und der grammatischen Bedeutung näher zu bestimmen. 56 Ein anschauliches Beispiel für diese Strategie findet sich in der AG-70 (37). Ohne eigens darauf hinzuweisen, daB die von ihnen verwendeten Tertia comparationis auf verschiedenen Ebenen liegen, bestimmen hier LOPAIIN und ULUCHANOV die 'Wortbildungsbedeutung', indem sie sie einmal der 'lexikalischen' Bedeutung gegenüberstellen, von der gelte, daB sie einem einzelnen Wort oder einer Wortgruppe eigne, jedoch über keinen speziellen innerwörtlichen Ausdruck verfüge. Ferner unterscheide sie sich auch von der sogenannten grammatisch-kategorialen Bedeutung, die ausnahmslos in allen Wörtern eines gegebenen Redeteils repräsentiert sei. Die 'Wortbildungsbedeutung' soll hinsichtlich der beiden genannten Bedeutungstypen eine $Z$ wischenposition einnehmen, da sie einerseits wie die 'lexikalische' Bedeutung dem Wort im Ganzen eigne (und nicht einer einzelnen Wortform oder einigen Wortformen) und sie andererseits wie die grammatische Bedeutung zwar mit innerwörtlichen Mitteln, doch nur bei einem Teil der Wörter ausgedrückt werde, die zu dem gegebenen Redeteil gehörten.

Aufgrund dieser Uberlegungen könnte man die 'Wortbildungsbedeutung' als Sonderfall der 'eigentlich nominativen Bedeutung' (vgl. ZALIZNJAK 1967, 26) interpretieren; allerdings gibt die hier vorgenommene Gegenüberstellung von 'Wortbildungsbedeutung' und grammatischer Bedeutung insofern nicht viel her, als aus inr lediglich folgt, dab die 'Wortbildungsbedeutung' nichts mit den paradigmatischen Beziehungen zwischen den Wortformen eines Lexems zu tun hat und nicht zum Ausdruck der Relationen zwischen den konkreten Wortformen eines Textes dient (vgl. dazu

56 So unterschied bereits SAPIR (SÉPIR 1934, 68f, 78f) 'konkrete', 'derivationale' und 'relationale' Begriffe, wobei er allerdings die' 'konkreten' und die 'derivationalen' aufgrund ihres Unvermögens, die Beziehungen zwischen den konkreten Wortformen eines Textes auszudrücken, gleichsam 'in einen Topf steckte'. 
die Definition der 'grammatisch-kategorialen' Bedeutung in der AG-70, 302). Auch die Abgrenzung von der 'lexikalischen' 8edeutung hilft nicht viel weiter, da in der Grammatik nirgends gesagt wird, was man unter einer solchen zu verstehen habe. Aus diesem Grunde ist auch das Kennzeichen der 'intermediären Position' wenig aussagekräftig. 57

Dennoch verweist auch KUBRJAKOVA auf die besondere stellung der 'Wortbildungsbedeutung' zwischen der 'lexikalischen' und der grammatischen Bedeutung (vgl. KUBRJAKOVA 1980, 105f) und gelangt zu dem SchluB, daB es sich bei der 'Wortbildungsbedeutung' um einen neuen, selbständigen Bedeutungstyp handele, da sie sich prinzipiell gegenüber den beiden anderen Bedeutungen abgrenzen lasse (vgl. ebd., 82). Aufgrund dieser Prämissen definiert sie die 'Wortbildungsbedeutung' als "verallgemeinerte kategoriale Bedeutungen, die einen speziellen Ausdruck haben, jedoch nicht obligat sind" (KUBRJAKOVA 1974, 48; genau so in SEREBRENNIKOV 1972, 357). Ganz ähnlich begreift auch MANUCARJAN (1975, 238f) die Beziehungen zwischen den drei Bedeutungstypen.

Wie läßt sich nun das Verfahren beschreiben, mittels dessen die 'Wortbildungsbedeutung' für gewöhnlich 'extrahiert' wird? In der Regel wird zu diesem Zweck so vorgegangen, daB man alle Derivate, die man sich von Angehörigen eines und desselben Redeteils abgeleitet denkt und die über einen und denselben 'formanten' verfügen ${ }^{58}$, im Hinblick auf solche inhaltlichen Gemeinsamkeiten untersucht, die sie von inren Derivatemen unterscheiden (vgl. bspw. AG-70, 39; ZEMSKAJA 1973, 84; Russkij jazyk, 305; AG-80, I, 135). Dazu ein Beispiel: In der AG-70 (180) wird den Adjektiven, die mittels des Suffixes -n- von Substantiven abgeleitet sind, die 'allgemeine Wortbildungsbedeutung' "sich auf den Gegenstand, auf die Erscheinung beziehend, die mit dem

57 In der $A G-80$ wird ein solcher Oppositionsbildungsversuch bereits nicht mehr vorgenommen.

S8 Im Rahmen der Derivation versteht man unter einem 'Formanten' meist das Wortbildungsaffix; für den Bereich der Substantivierung von Adjektiven und Adverbien gelten als 'Formant' die entsprechenden Substantivflexionsendungen. - Von akzentuellen Veränderungen und von solchen im segmentphonematischen Bau des abgeleiteten Wortes ist im Zusammenhang mit dem 'formanten' gewöhnlich nicht die Rede (vgl. ULUCHANOV 1977, 10). 
motivierenden Wort benannt ist" zugewiesen. Dabei lassen es LOPATIN und ULUCHANOV jedoch nicht bewenden, sondern unterteilen die Angehörigen der Klasse 'desubstantivische Adjektive mit dem Suffix $-\underline{n}-{ }^{\prime}$ in thematische Gruppen, denen sie beispielsweise die 'Wortbildungsbedeutungen' "über das verfügend, durch das charakterisiert, was mit diesem [ableitenden - J.K.] Wort benannt ist", "die Eigenschaften dessen aufweisend, was mit diesem Wort benannt ist" oder "zu dem neigend, was mit diesem Wort benannt ist" zuordnen. Dabei bleibt unklar, auf welche Weise die letztgenannten 'Wortbildungsbedeutungen' von der 'allgemeinen Wortbildungsbedeutung' abgegrenzt werden. Neben diesen existieren noch ausdrücklich so bezeichnete 'spezielle Wortbildungsbedeutungen' wie z.B. "zur Erfüllung einer Handlung vorgesehen" ( $A G-70,181$ ) bei solchen Adjektiven, die von Substantiven mit 'Handlungsbedeutung' abgeleitet sind.

Uberaus unbefriedigend ist bei diesem Verfahren zum einen, daB die Gruppenbildung ganz beliebig vorgenommen und zumindest theoretisch so weit getrieben werden kann, daB jeder Angehörige der Klasse 'desubstantivische Adjektive mit dem Wortbildungssuffix -ñ-' eine Gruppe für sich bildet. Zum anderen ist, wie noch näher zu erläutern sein wird, füglich zu bezweifeln, daB die ganze Vielfalt der so eruierten 'Wortbildungsbedeutungen' tatsächlich, wie dies LOPATIN (vgl. 1977, 8f) und ULUCHANOV (vgl. 1970, 16; 1974, 76) sowie eine Reihe anderer Linguisten annehmen (vgl. bspw. KUBRJAKOVA (in SEREBRENNIKOV 1972, 357); MAKSIMOV 1975, 23; MANUČARJAN 1974, 519f; REVZINA 1969, 8; ZVEREV 1978, 75f u.v.8.) grundsätzlich affixal ausgedrückt ist.

Darüber hinaus läßt sich durchaus der Standpunkt vertreten, dab es sich bei den so verstandenen 'Wortbildungsbedeutungen' um nichts anderes handelt als um verallgemeinerte Explikationen der nichtgrammatischen Bedeutungen der Derivate als ganzer. Anschauliche Belege dafür finden sich auch bei MANUČARJAN (1978, $12 \mathrm{f}$ ), der die 'Wortbildungsbedeutung' genau wie LOPAIIN (1974, $48 \mathrm{f}$ ) als sich wiederholende bikomponentiale inhaltliche 'Summe' des ableitenden Wortes sowie des Derivationsaffixes begreift und beispielsweise dem Lexem nosil'šcik (Träger) die 'Wortbildungsbedeutung' "derjenige, der die mit dem ableitenden Wort 
bezeichnete Handlung durchführt" zuweist. Hier und auch an anderer Stelle (MANUĆARJAN 1974, 522) wird ganz deutlich, daB MANUČARJAN zWar immer von der 'Wortbildungsbedeutung' redet, tatsächlich aber in allgemeiner Form die gesamte Inhaltsseite von Derivaten expliziert.

Bisweilen (vgl. GOLOVIN 1966, 119) findet sich auch der versuch, die 'Wortbildungsbedeutung' sozusagen mittels einer 'Subtraktion' der Bedeutung des Derivatems von der des Derivats zu ermitteln. Bei dieser Vorgehensweise werden die inhaltlichen Unterschiede zwischen abgeleitetem und ableitendem Wort als 'Wortbildungsbedeutung' deklariert. Dieses Prinzip sei an einem Beispiel erläutert, wozu wir die Wortpase (a) umnyj (klug) - umnik (kluger Mensch), (b) ucebnyj (Lehr-) - uとebnik (Lehrbuch), (c) kofejnyj (Kaffee-) - kofejnik (Kaffeekanne), (d) konopljanyj (Hanf-) - konopljanik (Hanffeld) betrachten wollen. 'Zieht' man den Inhalt des Adjektiva von dem des mit inm korrelierenden Substantivs 'ab', so ergibt sich als 'Rest' etwa bei (a) 'Mensch', bei (b) 'Buch', bei (c) 'Kanne' und bei (d) 'feld'. Wenn man gleichzeitig nicht nur die Inhaltsseiten, sondern auch die Ausdrucksseiten der in Rede stehenden Wörter voneinander 'subtrahiert' (etwa den Stamm des jeweiligen Adjektivs von dem des entsprechenden Substantivs), so bleibt jeweils das Suffix -ik- übrig, und dann liegt es nahe, die so aufgefundenen 'Wortbildungsbedeutungen' gleich auch mit dem formelen 'Rest' zu korrelieren.

Gegen eine solche Subtraktionsprozedur wendet sich zu Recht JANKO-TRINICKAJA $(1963,84)$, da bei diesem Vorgehen eine Reihe von Affixen als polysem oder als durch Homonyme repräsentiert gelten müßten. Dieser Umstand bewirkt natürlich eine nicht zu unterschätzende Komplizierung bei der Beschreibung der Bedeutungen von Affixen (sofern man überhaupt danach trachtet, Af fixe mit einer eigenen, autonomen Bedeutung zu vergehen). Daher ist dieses Verfahren, ohne dab wir hier bereits Uberlegungen hinsichtlich seiner Adäquatheit anstellen wollen, schon in bezug auf die wünschenswerte Einfachheit der Deskription nicht als optimal einzustufen.

Die 'Wortbildungsbedeutung' wird daneben auch, und dies vor allem seitens der Vertreter der applikativ-generativen Richtung, 
als die Bedeutung aufgefabt, die sich aus dem Gesamt der sogenannten kategorialen Bedeutungen des Derivats und des Derivatems konstituiert (vgl. SOBOLEVA 1970, 11; 1980, 18; GINZBURG $1979,22 \mathrm{f}$; aber auch CHOCHLAČEVA 1976, 36). SIRŠ́V (1979, 110) kritisiert dieses Vorgehen unter anderem deshalb, weil seiner Meinung nach auf dieses Weise Wortbildungsrelationen zwischen Angehörigen eines und desselben Redeteils nicht beschrieben werden können. Dieses Argument verfängt jedoch nicht, da sich dann, wenn man mit der 'kategorialen' Bedeutung operieren will, durchaus beispielsweise bei der deadjektivischen Bildung von Adjektiven die 'Wortbildungsbedeutung' 'Merkmal, das in einer Beziehung zu einem Merkmal steht' ansetzen läBt. Gegen ein solches Vorgehen, sofern es allein die Grundlage der Beschreibung abgeben soll, spricht zum einen die oben ( $S .21$ ) angeführte Kritik und zum anderen der Umstand, daB es, jedenfalls für die Zwecke der vorliegenden Arbeit, ein zu grobes Raster liefert.

Auch KUBRJAKOVA (1974, 149f; 1978, 36f; 1981, 101f) teilt in etwa die Auffassung, die 'Wortbildungsbedeutung' sei das Resultat des Einwirkens einer 'kategorialen' Bedeutung auf eine andere und somit das gleichzeitige Aufeinandertreffen von mindestens zwei 'kategorialen' Bedeutungen innerhalb der Grenzen einer Nomination. Nahezu zwangsläufig folgt daraus, daB das Wortbildungsaffix keine selbständige Bedeutung trage, sondern lediglich eine Derivationsbeziehung indiziere (vgl. KUBRJAKOVA $1980,116 f)$. An anderer Stelle läBt KUBRJAKOVA (1980, 115; 1981, 103f) jedoch erkennen, daB sie 'Wortbildungsbedeutung' und 'Zusammenwirken zweier 'kategorialer' Bedeutungen' nicht konsequent gleichsetzt, sondern die 'Wortbildungsbedeutung' als 'semantische Etikette' begreift, die den Typ der Relation zwischen zwei 'kategorialen' Bedeutungen in Ubereinstimmung mit den auBersprachlichen Relationen zwischen Gegenstand und Gegenstand, Gegenstand und Handlung etc. präzisiert.

Die Auffassung, daB Wortbildungsaffixe für sich allein genommen keine eigenständige Bedeutung trügen, findet sich nicht etwa nur bei BARTOŚEvIĆ $(1972,86)$, der dem Derivationsaffix, indem er es als "nur eine der Komponenten, die ausschlieblich die äuBere Seite des Wortes formieren" auffaBt, die Zeichentiaftigkeit abspricht, sondern, wie schon gezeigt, auch bei Vertre- 
tern der applikativ-generativen Richtung und mit Einschränkungen auch bei KUBRJAKOVA. Bereits BUDAGOV (1958, 202), LEVKOVSKAJA (1962, 231), JANKO-TRINICKAJA (1963, 87f) sowie in jüngerer Zeit auch JANCENECKAJA $(1979$, 139) halten dafür, daB man in bezug auf das Derivationsaffix nur dann von Inhalten reden sollte, wenn es sich mit den übrigen Strukturelementen des Derivats verbindet. Diese Auffassung läbt sich so interpretieren, daB das Affix über eine Bedeutung verfügen soll, die sich ausschlieblich innerhalb des intrawörlichen Kontextes in jeweils bestimmter Weise realisiert.

Im Prinzip ähnlich geht MANUCARJAN (1975, 192f) vor, der zwar dem Affix (in seiner Terminologie dem 'Derivator') von vornherein eine bestimmte Bedeutung zuweist, deren aktuelle Konkretisierung aber von inner- und auBerwörtlichen Kontexten abhängig sein könne. So unterscheidet er invariative Bedeutungen, die sich weitgehend unabhängig von den Kontexten realisieren, in die sie eingebettet sind, und variative Bedeutungen, für die dieses Independenz vom äuBeren Kontext nicht gilt. Den Umstand, daB in den Beispielen (a) dubovyjstol (Eichenstuhl), (b) dubovaja rošça (Eichenhain) und (c) dubovyj list (Eichenblatt) der 'Derivator' -ov- seiner Meinung nach einmal die Bedeutung 'aus etwas Bestimmtem gefertigt' (a), ein anderes Mal 'sich aus etwas Bestimmtem zusammensetzend' (b) und bei (c) schlieblich die Bedeutung 'auf etwas Bestimmtem wachsend' annehmen kann, führt MANUČARJAN in erster Linie auf den 'syntaktisch-lexikalischen' Kontext zurück, also darauf, mit welchem Substantiv das Adjektiv dubovyj jeweils kollokiert.

Faßt man nun die wichtigsten Punkte des bisher Dargelegten zusammen, so ergibt sich im einzelnen folgendes: Die 'Wortbildungsbedeutung' gilt als selbständiger Bedeutungstyp, der sowohl mit der 'lexikalischen' wie auch mit der grammatischen Bedeutung Gemeinsamkeiten aufweist. Beschrieben wird sie einmal als der (invariative) inhaltliche Unterschied zwischen dem Gesamt aller Derivate mit einem und demselben formanten und ihren entsprechenden Derivatemen, die, genau wie die abgeleiteten Wörter selbst, jeweils einem bestimmten Redeteil angehören. Ein anderes Mal gilt die 'Wortbildungsbedeutung' als inhaltliche Differenz zwischen je für sich genommenen Derivaten und ihren 
Derivatemen, was die 'Extraktion' ganz spezieller 'variativer Wortbildungsbedeutungen' gestatten soll. Daneben wird die 'Wortbildungsbedeutung' aber auch als bikomponentiale Summe der Inhalte des ableitenden Wortes und des Derivationsaffixes begriffen oder mit der Uberlagerung der 'kategorialen' Bedeutungen von Derivatem und Derivat gleichgesetzt. Schlieblich findet sich noch der Gedanke, die 'Wortbildungsbedeutung' sei dasjenige Inhaltselement innerhalb des abgeleiteten Wortes, das die Beziehungen zwischen den genannten 'kategorialen' Bedeutungen näher beschreibe.

Ein ähnlich heterogenes Bild ergibt sich auch in bezug auf das Derivationsaffix, das einmal als Träger der 'Wortbildungsbedeutung', von anderen Linguisten jedoch lediglich als Indikator für das Vorhandensein einer Derivationsbeziehung angesehen wird, wobei die 'Wortbildungsbedeutung' dem derivierten Lexem als Ganzem eigne. Wieder andere sprechen dem Affix keinerlei zeichenhafte Eigenschaften und damit auch keine eigentliche Funktion im Rahmen des Derivats zu.

Im AnschluB an diesen kurz gefabten Uberblick ist zu fragen, ob es widerspruchsfrei möglich ist, innerhalb der nichtgrammatischen Bedeutung des abgeleiteten Wortes gegebenenfalls eine 'Wortbildungsbedeutung' auszusondern, die somit tatsächlich als besonderer Bedeutungstyp begriffen werden könnte.

Auffällig ist die häufig unscharfe Trennung $z$ wischen der Bedeutung des Derivationsaffixes (sofern eine solche angesetzt wird), der 'Wortbildungsbedeutung' und der 'lexikalischen' Bedeutung. So unterscheidet ZEMSKAJA $(1973$, 184) zwar die Bedeutung des Derivationsaffixes von der 'Wortbildungsbedeutung', die sie als die Bedeutung des Affixes zuzüglich der des Stamms des ableitenden Wortes aufaBt, erweist aber damit ungewallt ganz eindeutig, daB es sich bei der'Wortbildungsbedeutung' um eine nicht klar umrissene Gröbe handelt, da sich eine so beschriebene 'Wortbildungsbedeutung' als völlig identisch mit der nichtgrammatischen Bedeutung des Derivats herausstellt.

Wie fließend die Grenzen zwischen diesen beiden Bedeutungstypen sind, kommt auch bei LOPATIN und ULUCHANOV (1978, 48) zum Ausdruck. Bei ihnen gilt die Bedeutung des 'Formanten' (mit 'Formant' sind an dieser Stelle ausdrücklich alle formalen Mit- 
tel gemeint, die das Derivat vom Derivatem unterscheiden) als nicht existent auBerhalb der Verbindung mit dem Stamm des ableitenden Wortes, in der allein sich die 'Wortbildungsbedeutung' realisiert. Der 'Formant' drückt also die 'Wortbildungsbedeutung' aus, von der gilt, daB sie dem Wort als Ganzem eignet. Da die nichtgrammatische Bedeutungdes Derivats ganz genau so durch die Kombination dieser beiden Elemente ausgedrückt wird, bleibt unklar, worin nun eigentlich der Unterschied besteht. Hier (und auch bei anderen Linguisten (vgl. etwa MANUCARJAN (1975, 74)) erscheint vornehmlich das Verhältnis zwischen der sogenannten Wortbildungsbedeutung und der nichtgrammatischen Bedeutung des Derivats als nicht genügend bedacht.

Für den Bereich der Wortbildung kann es, zumindest in der vorliegenden Arbeit, nur darum gehen, aus den zahlreichen, prinzipiell oft nicht odernur sehr schwierig in ihrer Gesamtheit erfaßbaren Inhaltslementen von Derivaten (und um im Grunde nichts anderes handelt es sich bei den $z . B$. in der $A G-70$, in der AG-80 oder bei MANUCARJAN (1975) beschriebenen 'Wortbildungsbedeutungen'), die einem und demselben strukturtyp, beispielsweise 'Substantivstamm + Formant $X$ ' zugeschrieben werden, diejenigen zu isolieren, die allen (z.B. in OŽEGov "l977 fixierten) speziellen Bedeutungen nicht widersprechen. Die jeweiligen konkreten inhaltlichen Ausprägungen dieser Derivate können als durch deren verschiedenen konkreten Gebrauch bedingt erklärt und deren Beschreibung dem Lexikographen überlassen werden ( vgl. dazu auch JANKO-TRINICKAJA $(1963,89)$, die zumindets implizit diese Auffassung teilt).

Ferner wird hier die Ansicht vertreten, daB das abgeleitete Wort in inhaltlicher Hinsicht zwar eine komplexe Benennung, aber doch eine Gesamtheit darstellt, deren Inhaltselemente den einzelnen Ausdruckselementen nicht ein-eindeutig zugeordnet werden können (vgl. etwa iskusstvennik = Kind, das künstlich ernährt wird). Bei der Bildung eines 'neven' Wortes handelt es sich also nicht einfach darum, daB der Stamm des wortes, das wir als das ableitende ansehen, mit einem Derivationsaffix verknüpft wird, wobei beide Komponenten eine bestimmte 'semantische Belastung' aufweisen. Das Affix (die Kombination von Affixen) wird aus diesem Grunde hier prinzipiell nicht mit irgend- 
welchen Inhalten korreliert. Stattdessen bescheiden wir uns damit, zu sagen, daB die Funktion des Affixes weniger darin besteht, diesen oder jenen selbständigen Inhalt im Lexikon einer Sprache zu fixieren, sondern vielmehr darin, die Umbildung geschlossener Konfigurationen von Inhaltselementen in andere, mit ihnen in bestimmter Weise korrelierende Konfigurationen zu signalisieren.

Mit anderen Worten: In der vorliegenden Arbeit sollen (ähnlich wie bei KUBRJAKOVA (1980, 111)) die Beziehungen zwischen dem ableitenden Wort und dem Derivationsaffix als einer Konstituente des abgeleiteten Wortes als Projektion der Relationen verstanden werden, die das Individuum in der es umgebenden Welt entdeckt und die es im Nominationsakt fixieren will. Die Funktionen von abgeleitetem Wort und 'Formant' sind unterschiedlich, da letzterer (unter dem hier sämtliche Mittel verstanden werden sollen, die den Stamm des Derivats von dem des Derivatems unterscheiden) einen Bedeutungszuwachs anzeigt, wohingegen der im Derivat enthaltene Stamm des ableitenden Wortes zum einen der Bewahrung derjenigen Komponenten aus dem Komplex der nichtgrammetischen Inhaltselemente des Derivatems dient, die für den neven Namen unerläBlich sind, zum anderen aber möglicherweise bestimmte mit dem Derivatem verknüpfte Assoziationen hervorruft. 59

\subsubsection{Morphonologische Alternation}

Mit dem bisher Ausgefühten, insbesondere mit der Bestimmung dessen, welches von zwei miteinander in einer Derivationsbeziehung stehenden Lexemen als das ableitende und welches als das abgeleitete gelten soll, wurde die Grundlage dafür geschaffen,

59 Der Sachverhalt, der hier mit dem Terminus 'Assoziation' bezeichnet werden soll, sei an einem Beispiel exemplifiziert: Bei weitem nicht immer gelingt es, alle Inhaltselemente eines abgeleiteten Wortes etwa mittels einer 'Addition' seiner kleinsten bedeutungstragenden Elemente zu gewinnen. Vgl. bspw. meлтуха, ein Wort, das von желтый abgeleitet gedacht werden kann. Mit 'gelb' assoziiert man in diesem Fall bekanntlich 'farbe der Haut'; darüber hinaus kann offensichtlich auch die Inhaltskomponente 'Erkrankung' (oder wie auch immer) weder mit mesT-, noch mit -yXdirekt korreliert werden. Diese Inhaltselemente sind es, die hier 'Assoziationen' genannt werden sollen. 
uns nun mit den sogenannten morphonologischen Erscheinungen ouf dem Gebiet der deadjektivischen Wortbildung beschäftigen zu können. Dabei ist es insofern unumgänglich, wissen zu müssen, welches das ableitende wort ist, da dieses für die Beschreibung der morphonologischen Veränderungen, die sich zwischen Derivatem und Derivat ansetzen lassen können, die Ausgangsgröße für die Bestimmung der Basisform abgeben soll.

Als Basis- oder Bezugsform wird in der vorliegenden Arbeit somit nicht die gewählt, die in der AG-80 (I, 134) 'motivierender Stamm' (= Stamm des Derivats abzüglich des Wortbildungsaffixes) genannt wird, da so die Mittel, die auBer dem Affix und der vom ableitenden Adjektiv eingebrachten Stammkomponenten am Ausdruck von Derivationsbeziehungen beteiligt sind, keine Berücksichtigung finden. Dieses Beispiel veranschaulicht, daB die Qualität dieser Mittel entscheidend von der Beschaffenheit der Bezugsform abhängt. In diesem Zusammenhang wird die Basisform in der Regel unter dem Gesichtspunkt ausgewählt, daß sie "in möglichst vielen fällen einen sicheren SchluB auf die jeweils in Frage kommende Konfiguration morphonologischer Alternationen [...] zuläBt oder zumindest die Zahl der Möglichkeiten in starkem MaBe begrenzt" (LEHFELDT 1978b, 33). Bei der deadjektivischen Wortbildung bietet sich der Stamm des ableitenden Adjektivs, also das um die flexionsendung verkürzte Adjektiv, an. Dieses Gröbe, die sich leicht (vgl.o.S. 32) aus dem Lexikoneintrag ermitteln läBt, hat jedoch - wie im empirischen Teil deutlich werden wird - den Nachteil, nicht sehr prädiktiv zu sein. Sofern man aber nicht insgesamt eine Vielzahl unterschiedlicher Basisformen erhalten will, indem man entweder ous jeweils wenigen hinsichtlich bestimmter Eigenschaften gleichen Adjektiven oder gar aus jedem einzelnen Adjektiv eine Bezugsform 'extrahiert', gilt dieser Einwand auch für andere denkbare Modellierungen obleitender Adjektive, wenn von ihnen gelten soll, daB sie alle oder jeweils sehr viele Adjektive des Russischen abdecken. Daher wird im empirischen Teil dieser Arbeit der Stamm des ableitenden Adjektivs als Bezugsform gewählt.

Was die Behandlung der Morphonologie und ihre Stellung innerhalb der Grammatik einer Sprache betrifft, kann es hier nicht darum gehen, einen umfassenden Uberblick über die verschieden- 
artigen strukturalistischen, deskriptivistischen oder generativistischen Ansätze innerhalb dieses Bereiches zu leisten. Ein in fast allen mir bekannten Arbeiten immer wieder auftauchender Aspekt verdient jedoch eine kurze Erörterung:

Bereits BAUDOUIN DE COURTENAY unterteilte sämtliche Alternationen in zwei Klassen, indem er die sogenannten neophonetischen Alternationen oder Divergenzen als kombinatorisch bedingte Alternationen der Modifikationen eines und desselben Phonems von den nichtneophonetischen oder paleophonetischen Alternationen abhob, die er als Relikte der geschichtlichen Entwicklung einer Sprache einstufte, und von denen ein Teil (die sogenannten Korrelationen) fähig sein sollte 'morphologische Kategorien' zu unterscheiden (vgl. BODUEN DE KURTENE 1963, 295-312). Diese strenge Zweiteilung findet sich wieder in den Arbeiten von Vertretern der Moskauer Phonologischen Schule (vgl. etwa REFORMATSKIJ 41967, 276f; 1975, 116f; KUZNECOV 1952, 62 Oder AVANESOV, SIDOROV 1945, 75f) sowie beispielsweise auch bei BERNŠTEJN (1968,44), LOPATIN (1977, 313), MAKAEV, KUBRJAKOVA $(1969,105)$, MEL'CUK $(1975,29)$ und vielen anderen.

Hauptargument für dieses Differenzierung ist regelmäßig der Faktor einer unterschiedlichen Bedingtheit der entsprechenden Alternationen. So gelten die von BAUDOUIN so genannten neophonetischen Alternationen als durch die 'phonologische' und die paleophonetischen ols durch die 'morphologische' (bisweilen auch: 'grammatische') Umgebung 'hervorgerufen'60 (vgl. neben den bereits genannten Autoren etwa auch ĆURGANOVA 1973, 40; BULYGINA 1977, 216f; IL'INA 1980, 27; OLIVERIUS 1976, 61).

In der vorliegenden Arbeit wird unter einer 'morphonologischen Veränderung' nicht nur jeglicher für das Russische regelmäBige "Wechsel eines Phonems mit einem anderen, mit einer Pho-

60 "Allgeme in wird das Morphem definiert als kleinste bedeutungstragende Einheit des Sprachsystems, als Klasse komplementär distribuierter Morphe mit gleicher Bedeutung, die durch Allomorphe realisiert und durch Phoneme (bzw. Prosodeme) lautlich repräsentiert wird" (LEWANDOWSKI 1975, 426). - Der Morphembegriff tauchte in der vorliegenden Arbeit insbesondere deshalb noch nicht auf, weil das Postulat der inhaltlichen Gleichheit der das Morphem konstituierenden Morphe zumindest fragwürdig erscheint. 
nemgruppe oder mit Null" (ARDENTOV 1979, 88) verstanden ${ }^{61}$, der sich beim Vergleich des Stamm des ableitenden Wortes mit dem des abgeleiteten Wortes (abzüglich des Wortbildungsaffixes) beobachten läBt, sondern auch bestimmte phonematische Erweiterungen oder Verkürzungen der Basisform, und dies unabhängig davon, ob diese Veränderung phonologisch-distributionell oder anders bedingt ist, was immer dies auch bedeuten mag.

Für die grundsätzliche Gleichbehandlung aller denkbaren Arten morphonologischer Veränderungen sprechen vor allem zwei Gründe:

(1) Nur mit erheblichen Vorbehalten kann man sich etwa zu der Aussage verstehen, die in einem Wortpar wie ruka (Hand) rućnoj (Hand-) auftretende Konsonantenalternation / $/ \sim / C, /$ signalisiere "unabhängig von ihrem phonetischen Unterschied, daB diese Elemente [ruk-, ruE-; J.K.] ouf der morphologischen Ebene identisch sind" (AChMANOVA 1966, 53). Genausowenig trifft es zu, daB im falle steklo (Glas) [st,okl-ó] - o stekle (über das Glas) [st,okl,-é] sich die "Varianz der Morphe (vom morphologischen Standpunkt aus) als bedeutungslos" (MAKAEV, KUBRJAKOVA 1969,104 ) erweist.

In diesen beiden 2 itaten dokumentiert sich, wie ich meine, der Hauptfehler innerhalb der gängigen Morphemdefinitionen. Von den komplementär distribuierten Morphen [ruk] - [ruć,] sowie [st,okl] - [st,okl,] kann nicht ernstlich behauptet werden, sie seien funktionell identisch. So signalisiert die Wurzel [ruč,] beispielsweise, dab sich die Endungen der sogenannten 2. Deklination niemals direkt mit ihr verbinden können; die wurzel [st,okl,] wiederum informiert unter anderem darüber, dab sie von allen Wortformen des Lexems steklo ausschlieblich in der des Präpositiv Singular repräsentiert sein kann, oder, anders gesagt, daB sie die Endung des Präpositiv Singular 'fordert'.62

61 Die Auffassung, daß bei der 'morphonologischen Alternation' gerade ein Phonemwechsel stattfindet, wird von den meisten Linguisten geteilt. Eine Gegenposition vertritt allerdings beispielsweise a IVERIUS (1976, 58), der nicht von Phonemalternationen, sondern von Alternationen der Allomorphe eines gegebenen Morphems redet, was aber, wie bei OLIVERIUS (ebd., 62) selbst deutlich wird, deskriptiv auf dasselbe hinausläuft.

62 Die hier beschriebenen Veränderungen haben selbstverständlich ols Spe- 
Dieses Beispiel belegt, daB es sich auch bei den phonologisch-distributionell bedingten durchaus nicht um "bedeutungsleere Alternationen" (8ULYGINA 1977, 233; MEL'ČUK 1975, 28) handelt, da ihnen offensichtlich diakritische Funktionen zukommen. 63

(2) Ein anderes Argument für eine in funktionaler Hinsicht prinzipielle Gleichstellung der sogenannten phonologisch bedingten und der nichtphonologisch bedingten Alternationen ist dies, daB "es Fälle von Alternationen geben kann, in denen es zu einem gegebenen Zeitpunkt prinzipiell nicht möglich ist, dieses Einstufung in begründeter form durchzuführen" (LEHFELDT 1981, 52). Mit anderen Worten: Bei den als morphonologisch qualifizierten Alternationen handelt es sich möglicherweise um solche Verbindungen des alternierenden Phonems mit dem (den) Phonem(en) seiner Umgebung, die von der phonologischen Ebene der Sprache potentiell als phonologisch-distributionell 'erlaubte' Phonemkombinationen realisiert werden können.

Die beiden genannten Uberlegungen lassen es als gerechtfertigt erscheinen, in der erwähnten Hinsicht im empirischen Teil der Arbeit 'neue Wege' zu beschreiten. 64

(62) zialfälle zu gelten, da morphonologische Alternationen durchaus nicht immer eine diakritische Funktion tragen. So signalisiert im Rahmen der Präsensflexion etwa der Wechsel /ova/ /uj/ z.8. bei [torgova-t,] [torgujj-u], [torgúj-os] etc. nichts über Person und Numerus der jeweiligen Endung, da er in allen Präsenswortformen dieses Verbs vorkommt.

63 Diese diakritische Funktion wird z.B. auch von MAKAEV, KUBRJAKOVA (1969, 105), von AVANESOV, SIOOROV $(1945,75)$ oder von OLIVERIUS (1976, 31) erkannt, ohne daß dies jedoch zu einer Gleichbehandlung der phonologisch-distributionell und der anders bedingten Alternationen in dieser Hinsicht führen würde.

64 Nicht zur Morphonolgie zähle ich (entgegen REFORMAISKIJ 1975, 118; BERNŚTEJN 1968, 50) die suprasegmentalen Alternationen, denen ein eigenes Kapitel gewidmet sein wird. 
2. EMPIRISCHE ANALYSE DER DEADJEKTIVISCHEN WORTBILDUNG DES RUSSISCHEN

Die im ersten Hauptkapitel der Arbeit getroffenen Festlegungen sollen nunmehr hinsichtlich ihrer praktischen Tauglichkeit überprüft werden. Der empirische Teil setzt jedoch nicht sogleich mit dem bereits in der Einleitung kurz erläuterten Analyseschritt ein, sondern mit einer Klassifikation der Gesamtheit der Adjektive des Russischen aufgrund bestimmter inhaltlicher Merkmale, an die sich eine ähnlich geartete Darstellung der Inhaltsseite der deadjektivischen Redeteile anschliebt. Erst danach wird die Behandlung der Mittel erfolgen, die zum Ausdruck der letztgenannten Inhaltselemente beitragen.

Welchem Erkenntnisinteresse dient dieses Vorgehen, und warum erfolgen die genannten Klassenbildungen nicht aufgrund formaler Merkmale, eine Aufgabe, die sich ohne 2 weifel leichter bwerkstelligen liebe?

Aus der in der Einleitung formulierten Aufgabenstellung folgt unter anderem, daB bei der empirischen Untersuchung eines der Ziele sein muß, festzustellen, in welcher Weise die nichtgrammatischen Inhaltselemente von Lexemen, die im Russischen dem Redeteil 'Adjektiv' zuzuordnen sind, in anderen Lexemen desselben Redeteils oder anderer Redeteile verändert rekurrieren. Der Grund dafür, daB die engestrebte Klassifikation gerade unter inhaltlichen Gesichtspunkten erfolgen soll, liegt somit darin, dab anzunehmen ist, die Derivation sei vor allem ein Verfahren, nichtgrammatische Inhaltselemente von Lexemen (in unserem Fall von Adjektiven) ous kommunikativen Erfordernissen heraus auf bestimmte Weise in anderen Lexemen zu modifizieren. Da sich a priori vermuten läBt, daB nicht jedes Adjektiv des Russichen als Derivatem fungiert und somit eine Differenz zwischen potentiell ableitenden und tatsächlich ableitenden Adjektiven besteht, erfolgt die oben beschriebene Zerlegung eines Korpus der Adjektive des Russischen, wobei von der Annahme ausgegangen wird, die so gewonnenen Teilmengen hätten in systematischer Weise etwas mit der deadjektivischen Derivation zu tun: Von Adjektiven mit bestimmten typisierten Inhaltselementen können lexeme mit anderen Inhaltselementen abgeleitet gedacht 
werden. - Der hier formulierte Untersuchungsgegenstand ist zum Abschlub des synthetischen Teils dieser Arbeit zu behandeln.

Grundlage für die unten vorgenommene Klassifikation der Adjektive sind vor allem diejenigen Kapitel in der AG-70 sowie in der AG-80, die die Wortbildung der Adjektive zum Gegenstand haben oder genauer, die in ihnen enthaltenen Beschreibungen der 'Wortbildungsbedeutungen' abgeleiteter Adjektive.

Für die Einteilung der deajektivischen Redeteile kommen zum einen die in der $A G-80$ im AnschluB an die Behandlung der Wortbildung eines jeden Redeteils zusammen mit den (affixalen) Mitteln zu deren Ausdruck noch einmal gesondert aufgelisteten 'Wortbildungsbedeutungen' der derivierten Substantive ( $A G-80$, I, $256 \mathrm{ff}$ ), Adjektive (ebd., $331 \mathrm{ff}$ ), Verben (ebd., $393 \mathrm{ff}$ ) und Adverbien (ebd., $409 \mathrm{ff}$ ) in Betracht. Zudem werden auch die von MANUČARJAN (1975, $338 \mathrm{f}$ ) so genannten deadjektivissch-substantivischen und die deadjektivisch-verbalen 'Wortbildungskategorien' (worunter nach Auffassung des Autors die 'Wortbildungsbedeutungen' der deadjektivischen Substantive/Verben zu verstehen sind) berücksichtigt.

Die 'Wortbildungsbedeutungen' der Akademiegrammatiken sowie die MANUCARJANBchen 'Kategorien' heiBen in der vorliegenden Arbeit 'verallgemeinerte Explikationen der nichtgrammatischen Inhaltselemente von Derivaten' und sind ersteren gegenüber mehr oder weniger stark verändert, d.h. erweitert oder (besonders in bezugauf die deadjektivischen Redeteile) zusammengefaBt und vereinfacht. Zu vermerken ist auBerdem, daB bei der Behandlung der abgeleiteten Adjektive die in der AG-70 sowie in der $A G-80$ verfolgte Systematik an den unten näher bezeichneten Stellen nicht übernommen werden konnte.

Der Begriff der allgemeinen Explikation wird hier weder definiert noch operationalisiert, sondern lediglich in einen Zusammentang mit den Begriffen 'Wortbildungsbedeutung' und 'Kategorie' gestellt, die ihrerseits anhand von Beispielen eingeführt wurden. Heuristisch ist so vorzugehen, daB solche lexeme, die Vertreter eines und desselben Redeteils darstellen und als nach einem bestimmten Muster gebildet gelten können, hinsichtlich ihrer (z.B. in OŽEGOV "'l977 fixierten) Bedeutungen miteinander verglichen werden. Dabei ist eine Bedeutung zu ermitteln, 
die allen individuellen Bedeutungen nicht widerspricht. Da dieses Vorgehen aus bestimmten Gründen (s.u.) nicht operationalisiert werden kann, liegt auf der Hand, dab die formulierung dieser Bedeutung unter Umständen von Linguist zu Linguist verschieden ausfallen kann. Bei der in Rede stehenden Bedeutung handelt es sich um die allgemeine Explikation, der die Lexeme der jeweils analysierten Menge und gegebenenfalls auch solche, die nach einem anderen Muster gebildet sind, zugeordnet werden. Allgemein läßt sich die Heuristik somit folgendermaBen beschreiben: Von der form zum Inhalt und dann (im synthetischen Teil der Arbeit) wieder zur Form bzw. zur Deskription derselben. Jede einzelne Explikation wird im folgenden aus Gründen der Übersichtlichkeit mit nur einem Beispiel versehen, wobei die gewählten Lexeme jeweils in Klammern mit ihrer pragmatischen Häufigkeit versehen sind. Schlieblich sei noch angemerkt, dab die allgemeinen Explikationen sämtlich auf der natürlichen Sprache basieren, da "in den Arbeiten, die eine Beschreibung der Semantik zum Inhalt haben, noch keine Sprache zur Deskription von Wortbedeutungen entwickelt wurde. Eine semantische Metasprache [...] existiert noch nicht" (ULUCHANOV 1977, 17). Allerdings gibt es beachtenswerte versuche, diesen unbefriedigenden Zustand zu überwinden (vgl. etwa APRESJAN 1969a, 1969b, 1974; KOTELOVA 1974; MEL'CUK 1963; MEL'ČUK, ŽOLKOVSK1J 1969). 
2.1. Zerlegung der (offenen Menge der) Adjektive des Russischen aufgrund der allgemeinen Explikationen ihrer nichtgrammatischen Inhaltselemente

D-I Aus dem gewählten Korpus der Adjektive des Russischen soll in der vorliegenden Arbeit die erste Gruppe von solchen konstituiert sein, die sich als nichtabgeleitet denken lassen.

Während die abgeleiteten Adjektive (Gruppen D-II bis D-IV und (D-)l bis (D-) 3 ) die Eigenschaften der mittels ihrer spezifizierten Denotate unter Bezug auf andere Denotate, also mittelbar, benennen, liegt bei den Adjektiven der Gruppe D-I ( $z . B$. bei красный, далекий, молодой, вапный, тяжелый, жадный, мудрый) immer eine unmittelbare Denomination vor. Diese Adjektive lassen sich zwar hinsichtlich rekurrenter nichtgrammatischer Inhaltselemente zu Untergruppen zusammenfassen, doch führen solche Versuche zu keiner eindeutigen Abgrenzung von den abgeleiteten Adjektiven. So heiBt es beispielsweise in der AG-80 (541), Adjektive dieses Typs bezeichneten "solche Eigenschaften, die unmittelbar von den Sinnesorganen wahrgenommen werden: farbliche, räumliche, zeitliche, physische und andere qualifizierende Merkmale, charakterliche und geistige Eigenschaften". Wie man sich jedoch anhand der im folgenden angeführten Beispiele leicht überzeugen kann, treffen dieses Unterscheidungsmerkmale durchaus auch ouf die nachstehenden Adjektive zu.

D-II Die nichtgrammatischen Inhaltselemente desubstantivischer Adjektive können das mittels ihrer spezifizierte Denotat kennzeichnen als

1. eine Eigenschaft aufweisend, die dem mit dem ableitenden Substantiv Benanten zugeschrieben wird.

1 Wenn hier von einer Zerlegung der Adjektive und später von einer solchen der deedjektivischen Redeteile des Russischen gesprochen wird, so kann damit immer nur ein bestimmtes Korpus gemeint sein, de die Redeteile des Russischen eine offene Menge konstituieren. Das in dieser Arbeit gewählte Korpus ist durch die in ZALIZNJAK 1977 enthaltenen wörter und die in der $A G-80$ verwendeten Illustrationsbeispiele bestimnt, wobei a priori angenomen werden soll, daB sich die bei der Analyse unseres Korpus erzielten Resultate verallgemeinern lassen. - Das im folgenden vor den römischen Zahlen verwendete ' $D$ ' steht für 'Derivatemtyp'. 
Bsp.: зверь (77) - звериный (1)

2. mehr oder minder intensiv durch das mit dem ableitenden

Substantiv Benannte charakterisiert.

8sp.: глаз (1093) - глазастый (4)

3. dem mit dem ableitenden Substantiv Benannten eigen oder gehörig. Bsp.: дед (185) - дедов (3)

4. mit dem durch das ableitende Substantiv Benannte begabt. Bsp.: дар (24) - даровитый (2)

5. das mit dem ableitenden Substantiv Benannte verschaffend oder bietend. Bsp.: Комфорт (4) - комфортабельннй (3)

6. dem mit dem ableitenden Substantiv Benannten dienlich. Bsp.: империализм (112) - проимпериалистический (2)

7. unter Einwirkung des mit dem ableitenden Substantiv Benannten befindlich." Вsp.: власть (364) - подвластный (4)

8. sich zwischen Gegenständen solcher Art befindend oder ereignend, wie sie mit dem ableitenden Substantiv benannt sind." Вsp.: город (803) - мехдугородный (-)

9. durch ein Abwechseln des mit dem ableitenden Substantiv Benannten charakterisiert.

Bsp.: полоса (77) - черезполосный (-)

10. sich gegen das mit dem ableitenden Substantiv Benannte richtend. ${ }^{+}$

Bsp.: демократия (79) - антидемократический (7)

11. der mit dem ableitenden Personennamen verknüpften Richtung angehörig. Bsp.: Фейербах (-) - фейербахианский (-)

12. das mit dem ableitenden Substantiv Benannte in mehr oder minder starkem Maße enthaltend oder als differenzierendes Merkmal aufweisend." Вsp.: cepa (16) - сернистый (14)

13. kleiner als das mit dem ableitenden Substantiv Benannte. ${ }^{+}$ Bsp.: aтом (145) - субатомный (-)

14. das mit dem ableitenden Substantiv Benannte nicht aufweisend." Bsp.: Вина (46) - невинный (13)

+ Die hierher zu rechnenden Adjektive gelten in der $A G-70$ sowie in der AG-80 als deadjektivisch, eine Entscheidung, die sich jedoch bereits aufgrund der dort angeführten 'Wortbildungsbedeutungen' als fragwürdig erweist.

- Die Adjektive dieser Gruppe werden von den Verfassern der AG-70 und der AG-80 zum Teil als deadjektivisch eingestuft. 
15. temporal oder lokal dem mit dem ableitenden Adjektiv Benannten voraufgehend. "Bsp.: Война (825) - довоенный (14)

16. sich nach dem mit dem ableitenden Substantiv Benannten ereignend. Bsp.: Смерть (230) - посмертный (6)

17. in der Nähe des mit dem ableitenden Substantiv Benannten befindlich. Bsp.: Москва (-) - подмосковный (5)

18. entlang dem mit dem ableitenden Substantiv Benannten befindlich." Bsp.: граница (114) - пограничный (10)

19. neben dem mit dem ableitenden Substantiv Benannten oder um es herum befindlich." В8р.: луна (141) - окололунний (1)

20. auf dem oder oberhalb des mit dem ableitenden Substantiv Benannten befindlich. "Bsp.: Гроб (41) - надгробный (3)

21. Unterhalb des mit dem ableitenden Substantiv Benannten befindlich." Bsp.: ropa (242) - подгорный (2)

22. hinter dem oder jenseits des mit dem ableitenden Substantiv Benannten befindlich.

Bsp.: граница (114) - заграничный (11)

23. von dem mit dem ableitenden Substantiv Benannten ausgehend. Ввр.: глагол (4) - отглагольный (-)

24. sich durch das mit dem ableitenden Substantiv Benannte erstreckend." Bsp.: океан (134) - трансокеанский (1)

25. sich innerhalb der Grenzen des mit dem ableitenden Substantiv Benannten befindend oder ereignend.

Bsp.: партия (532) - внутрипартийный (6)

26. über die Grenzen des mit dem ableitenden Adjektiv Benannten hinausgehend. ' Bsp.: план (407) - сверхплановый (18)

27. sich auBerhalb der Grenzen des mit dem ableitenden Substantiv Benannten befindend oder ereignend.

Bsp.: наука (581) - вненаучный (1)

28. durch eine Gemeinsamkeit in bezug auf das mit dem ableitenden Substantiv Benannte bzw. durch eine gleichartige Beziehung zu inm charakterisiert. Bsp.: время (1856) - современный (261)

29 in Ubereinstimmung mit dem durch das ableitende Substantiv Benannte erzeugt, gemessen oder verteilt." Вsp.: доход (47) - подоходный (1)

D-III Die nichtgrammatischen Inhaltselemente deverbaler Adjek- 
tive können das mittels ihrer spezifizierte Denotat kennzeichnen als

1. durch das Vorliegen der mit dem ableitenden Verb benannten Handlung charakterisiert.

взр.: прерывать (12) - прерывистый (3)

2. zu der mit dem ableitenden Verb benannten Handlung neigend. Вsp.: ломать (45) - ломкий (2)

3. zur Erfüllung der mit dem ableitenden Verb benannten Handlung dienlich oder bestimmt.

Bsp.: ходить (415) - ходовой (11)

4. Für die mit dem ableitenden Verb benannte Handlung tauglich. Bsp.: дискутировать (s) - дискутабельный (-)

5. unfähig, die mit dem ableitenden Verb benannte Handlung auszuführen oder sich ihr zu unterziehen.

8sp.: возвратиться (23) - невозвратный (2)

6. fähig, Objekt der mit dem ableitenden Verb benannten Handlung zu sein. 2 8sp.: заводить (16) - заводной (2)

7. in einem Zustand befindlich, der als Resultat der mit dem ableitenden Verb benannten Handlung entstanden ist.

Bsp.: устать (72) - усталый (20)

8. durch das Nichtgegebensein der mit dem ableitenden Verb benanten Handlung charakterisiert. Вsp.: прерывать (12) - беспрерывный (7)

D-IV Die nichtgrammatischen Inhaltselemente der von Numeralia abgeleiteten Adjektive können die mittels ihrer spezifizierten Denotate kennzeichnen als

1. in einer bestimmten Zahlenreihe den mit dem ableitenden Grundzahlwort (vgl. Anm. 20, 23, S. $22 \mathrm{f}$ sowie Anm. 24, 26, S. 23) benannten Rang einnehmend.

Bsp.: пять (320) - пятьй (90)

2. Um sovielmal größer oder aus so vielen gleichartigen Teilen bestehend, wie mit dem ableitenden kollektivzahlwort (vgl.

2 Beziehungen der Art 'Subjekt der mit dem ableitenden Verb bezeichneten Handlung sein' (Bsp.: бajeTb (77) - бaлbHOй (134)) finden in diesem Zusammenhang keine Berücksichtigung, da in der ganz überwiegenden Mehrzahl der Fälle die Derivationsrichtung vom Adjektiv zum Verb anzusetzen ist. 
Anm. 20, S. 22) benannt sind.

Bsp.: Двое (63) - двойной (23)

D-V Die letzte Teilmenge ${ }^{3}$ schlieBlich wird von solchen Adjektiven gebildet, die, anders als die Angehörigen der Gruppen D-II bis D-IV, eine Eigenschaft der mittels inrer spezifizierten Denotate nicht nur als mit einer einzigen Erscheinung der auBersprachlichen Wirklichkeit in Beziehung stehend kennzeichnen. Die hier einzuordnenden Adjektive benennen solche Eigenschaften, die als jeweils auf bestimmte Woise mit den nichtgrammatischen Bedeutungen von zwei oder mehr Wörtern korrelierend aufgefaßt werden können. Die folgenden allgemeinen Explikationen konkretisieren diesen Typ:

Die nichtgrammatischen Inhaltselemente von Adjektiven, die auf der Ausdrucksseite durch die Stämme von zwei oder mehr Lexemen konstituiert sind, kennzeichnen das mittels ihrer spezifizierte Denotat als

1. eine Eigenschaft besitzend, die als Vereinigung der mittels dieser Lexeme benannten Merkmale aufgefaot werden kann.

Zu dieser Gruppe gehören Komposita (unter einem 'Kompositum' wird hier eine begriffliche Einheit verstanden, die das Ergebnis der Vereinigung zweier oder mehrerer in einem Text auch isoliert auftretender Wortformen zu einem einzigen Lexem darstellt), bei deren Konstituenten es sich um Adjektivstämme handelt (Bsp.: cлепоглухонемой).

2. eine Eigenschaft besitzend, die gleichermaßen zum einen mit demjenigen Merkmal korreliert, das mit dem die letzte Konstituente des Adjektivs bildenden Lexems benannt ist und zum anderen mit der Erscheinung (den Erscheinungen) der auBersprachlichen Wirklichkeit in Beziehung steht, die von dem Lexem (den Lexemen) bezeichnet wird (werden), dessen Stamm (deren Stämme) die erste(n) Konstituente(n) des Adjektivs ausmacht (ausmachen).

3 Deadverbiale Adjektive werden nicht angenommen, do zwischen den entsprechenden Adverbien und Adjektiven wegen der Gleichartigkeit ihrer (nichtgrammatischen) Inhaltselemente unechte Derivationsbeziehungen bestehen; gleiches gilt für von Komparativen 'abgeleitete' Adjektive. Depronominale Adjektive (zum 'Pronomen' vgl. Anm. 20, S. 22) bleiben dagegen wegen ihrer systemischen Singularität unberücksichtigt. 
Diese Gruppe umfaßt Komposita, die als zweite Konstituente ein Adjektiv und als erste einen nichtsuffigierten Substantivstamm (Bsp.: тазобедреннъй) oder einen verkürzten Substantiv- bzw. Adjektivstamm (Bsp.: сербохорватский) aufweisen. - In den Lexemen der Gruppen (1) und (2) stehen die jeweiligen konstituierenden Stämme in einer Beiordnungsbeziehung.

3. über das Merkmal verfügend oder durch das Merkmal charakterisiert, das mit dem die letzte Konstituente des Adjektivg bildenden Lexem benannt ist. Zusätzlich erfährt dieses Merkmal eine Konkretisierung durch die mit demjenigen Lexem (denjenigen Lexemen) bezeichnete( $n$ ) Erscheinung(en) der auBersprachlichen Wirklichkeit, dessen Stamm (deren Stämme) der letzten Konstituente voraufgeht (voraufgehen).

Hier handelt es sich um Komposita, die als zweite Konstituente ein nichtderiviertes Adjektiv und als erste einen Substantivstamm (Bsp.: помароопасный), einen Adjektivstam (Bsp.: белозеленый), verkürzte Stämme von Substantiven oder suffigierten Adjektiven (Bsp.: термостойкий), einen Zahlwortstamm (8sp.: Двууглекисльй) oder ein Adverb (Bsp.: вечнозеленый) oufweisen.

4. über die Substanz verfügend oder als durch die Substanz charakterisiert, die mit dem als Derivatem für die letzte Konstituente des Adjektivs anzusetzenden Lexem benannt ist. Eine zusätzliche Konkretisierung erfolgt durch die mit demjenigen Lexem (denjenigen Lexemen) bezeichnete(n) Erscheinung(en) der auBersprachlichen Wirklichkeit, dessen Stamm (deren Stämme) die erste(n) Konstituente(n) des Adjektivs ausmacht (ausmachen).

In diese Gruppe gelangen Komposita, deren zweite Konstituente von einem Substantiv deriviert gedacht werden kann und die als erste Konstituente einen Adjektivstamm (Bsp.: белозубьй), einen Zahlwortstamm (Bsp.: ОДноглазый), einen Substantivstamm (Bsp.: паросилоВOЙ) oder einen Verbalstamm enthal ten (Bsp.: КорноухиЙ).

5. zu der Handlung in Beziehung stehend, die mit dem als Derivatem für die letzte Konstituente des Adjektivs anzusetzenden Lexem benannt ist. Konkretisiert wird diese Beziehung durch die mit demjenigen Lexem (denjenigen Lexemen) bezeichnete(n) Erscheinung(en) der auBersprachlichen Wirklichkeit, dessen Stamm (deren Stämme) der letzten Konstituente voraufgeht (voraufgehen).

Hierher gehören Komposita, deren zweite Konstituente von einem Verb abgeleitet ist und die als erste Konstituente einen Substantivstamm (Bsp.: серосодерпаший) oder ein Substantiv im obliquen Kasus (Bsp.: 
умалишенный), einen Adjektivstamm (Bsp.: скоротечный), einen Zahlwortstam (Bsp.: Двустальный) oder ein Adverb (Bsp.: влередсмотряий) enthalten.

Auf der Basis der allgemeinen Explikationen der nichtgrammatischen Inhaltsseiten seiner Angehörigen wurde ein Korpus der Adjektive des Russischen mit Ausnahme der deadjektivischen in fünf Teilmengen zerlegt. Noch einmal sei erwähnt, dab unterschiedliche kontextuelle Bedeutungen, die einige Adjektive realisieren können (vgl. bspw. лесной in лесные заготовки, лесной дом, лесной пожар, лесной вопрос, лесная одежда, лесная зверь etc.), nicht berücksichtigt wurden, da es sich bei ihnen um Spezialfälle allgemeinerer Explikationen handelt.

Mittels einer Prozedur, die der oben angewandten ähnlich ist, soll nun in bezug auf die deadjektivischen Redeteile vorgegangen werden.

2.2. Die nichtgrammatischen Inhaltselemente der deadjektivischen Redeteile in allgemeiner Explikation

A. Substantive mit der Bedeutung eines abstrakten Merkmals

1. Merkmal als gesellschaftliche, politische, wissenschaftliche oder ästhetische Erscheinung oder Tendenz.

ввр.: реальный $(83)$ - реализм $(10)^{4}$

4 Bei MANUČARJAN (1975, 338ff) gehen dieser Explikation fünf weitere vorauf, u.z. (a) качество такого-то (Вsp.: амелость - "качество смелого"), (b) наличие того-то как качество (Вsр.: плановость - "наличие плана"), (c) степень наличия того-то как качество (Вsp.: доходность - "степень наличия доходов"), (d) способность или сюлонность к тому-то действио как качество (Вsp.: клейность - "стособность клеиться"; раздрахительность "склонность разирапаться") sowie (e) состаяние таного-то (Вsp.: влюбленность - "СОстояние влобленнот"). Beziehungen dieser Art werden in der vorliegenden Arbeit bekanntlich nicht berücksichtigt, de es sich bei ihnen entweder (u.z. bei (a) und bei (e)) um Relationen vom Typ 5 (vgl.o., S. 42) handelt oder um solche vom Typ 8 (ebd.). Dieser letztgenannte Beziehungstyp liegt in den Paaren (b) bis (d) vor, da die jeweiligen Derivate nur in formaler Hinsicht von den entsprechenden Adjektiven (חuлановый; доходньй; клейкий, раздрахительный) abgeleitet sind. Inhaltlich, und dies geht aus den Explikationen klar hervor, korrelieren sie mit denjenigen Substantiven bzw. mit denjenigen Verben, von denen sich ihrerseits die jeweiligen Adjektive deriviert denken lassen. In der AG-80 $(I, 267)$ wird vermerkt, daß bei solchen Lexemen, die dort als in einer 
2. Erscheinung der auBersprachlichen Wirklichkeit, die charakterisiert ist durch das Vorliegen des mit dem ableitenden Adjektiv benannten Merkmals in abgeschwächter Form. Вsp.: зеленый (216) - прозелень $(2)^{5}$

3. Merkmal als negativ bewertete Erscheinung oder Tendenz. Bsp.: обыденный (9) - обыденщина (1)

B. Substantive mit der Bedeutung des Merkmalsträgers ${ }^{6}$

1. Gesamtheit dessen, was sich durch ein bestimmtes Merkmal auszeichnet. Bsp.: старый (468) - старье (4)

2. Jemand (etwas), der (das) sich durch ein bestimmtes Merkmal auszeichnet. Bsp.: слепой (31) - слепец $(4)^{7}$

(4) 'Transpositionsrelation' befindlich charakterisiert sind und zwischen denen nach der in dieser Arbeit vorgenommenen Festlegung unechte Derivationsbeziehungen vorliegen, eine absolute Identität bezüglich der nichtgrammatischen Inhalte von Adjektiv und Substantiv gegeben sei. Wie wir jedoch gesehen haben, trifft dieser Spezialfall streng genommen nur auf die obigen Typen (a) und (e) zu.

5 Diese in der AC-80 ( $I, 267 f f$ ) 'transponierend' genannten Bedeutungen werden dort weiter untergliedert als dies bei MANUCARJAN der Fall ist (vgl. Punkt A.3.). Die dort ebenfalls angeführte Explikation "in starkem, intensivem MaB vorliegende Qualität eines Merkmals" soll hier nicht übernommen werden, da sie nur auf ein Derivat, U.z. auf rapanb zutrifft, das bei OŽEGOV ("1977, 175) zudem mit dem Vermerk mpocT. versehen ist.

6 Bei MANUČARJAN findet sich unter B.3. die Explikation "TOT, KTO ЯBляеTCF. такмм-то (лицом) или вообще имеет отношение $\mathrm{k}$ такаму-то (прелмету)" (Bsp.: глазник - "тот, кто имеет отношение к леченио глаза" (- глазной вpay)), unter B.5. die Explikation "TO, чTO является такм-то (препметом) или вообие имеет отношение к транспорту грузов" (- грузовой автомобиль)) und unter B.6. die Explikation "элемент язына, являюинйся такмм-то или вообще имеюиий отношение и таному-то (предмету)" (Ввр.: украинизм - "әлемент язына, имекиий отношение к уюраинскаму языу"). Aus den in Anmerkung 4 bezüglich der Punkte (b) bis (d) angestellten Uberlegungen heraus zählen auch Relationen der vorstehenden Art zum Typ ( 8 ) und fallen daher nicht in den 'Zustëndigkeitsbereich' der empirischen Untersuchung.

7 MANUCARJAN nennt unter B.7. die 'Kategorie' "яroдHoe pacterue, fRлgroщееся танм-то или вообще имекоее отношение к таному-то (препмету)", deren erster Teil ohne Schwierigkeiten bei B.3. untergebracht werden kann; Bsp.: черника (1) - "ятодное растенне, явлндыееся черным (473)". Der zweite Teil der Explikation bezieht sich offenbar auf Fälle wie: земляника (2) - "ягодное растение, имениее отношение к земле (1074)". Auch hier ist das entsprechende Adjektiv (зêU/яНОй(17)) nur formal das ableitende Wort. Inhaltlich korreliert земляника mit dem Lexem зexля, von dem sich wiederum ३evurяной deriviert denken läßt. Folglich haben wir 
Von den deadjektivischen Adjektiven finden diejenigen Aufnahme in die empirische Untersuchung, in denen die Bedeutungen der Derivateme modifiziert werden ( $v g 1 . A G-80,1,332$ ). ${ }^{8}$ Dabei sind die folgenden verallgemeinerten Explikationen zu unterscheiden:

(D-) 1. Gradation des mit dem ableitenden Adjektiv bezeichneten Merkmals.

1.1. Hervortreten des Merkmals in mäBiger Form. Bsp.: опасный (92) - небезопасный (1)

1.2. Hervortreten des Merkmals in verstärkter Form. Bsp.: хитрый (38) - хитрющий (1)

(7) es auch hier mit dem Beziehungstyp (B) zu tun. - In der AG-80 ( $1,256 f f$ ) werden den 'Wortbildungstypen' mit der Bedeutung 'Träger eines nichtprozessualen Merkmals' neun verschiedene 'Wortbildungsbedeutungen' zugewiesen, auf die, sofern sie nicht auf die drei genannten Explikationen zurückgeführt werden können, die in den Anmerkungen 6 und 7 vorgebrachten Einwände zutreffen.

8 Außerdem werden den deadjektivischen Adjektiven in der AG-80 ( $1,331 \mathrm{f})$ die nachstehenden 'präfixalen Konkretisationen der Bedeutungen einer Beziehung zu einem Gegenstand oder zu einem ProzeB' zugeordnet: (a) Konkretisierungen in lokal-temporaler Hinsicht (Bsp.: внутризаводсюой; послевоенњй); (b) Konkretisierung als über die Grenzen von etwas hinausgehend (Bsp.: Сверхчеловеческй); (c) Konkretisierung im Sinne eines Fehlens von etwas (Bsp.: бeзвольный); (d) Konkretisierung im Sinne einer negativen Einstellung gegenüber etwas (Bsp.: ПроТКВозаКОНнБй);

(e) Konkretisierung im Sinne einer positiven Einstellung gegenüber etwas (Bsp.: Iрозаладның); (f) Konkretisierung im Sinne einer in bezug auf etwas Bestimntes vorliegenden Gemeinsamkeit (Bsp.: СОВИнОВНЫЙ); (g) Konkretisierung im Sinne eines Uberflusses an etwas (Bsp.: 3areceHньЙ); (h) Konkretisierung in bezug auf die Herkunft, den Ursprung von etwas (Bsp.: отrлarajbłbí); (i) Konkretisierung in bezug auf eine in Übereinstimmung mit etwas befindliche Geregel theit (Bsp.: покварталь$\mathrm{Hb}(\bar{i})$; (k) Konkretisierung im Sinne einer (auch größenmäßigen) Untergeordnetheit unter etwas (Вsp.: подслизистый; субмиюронњҢ̆). - Bereits die in der AG-80 gebrauchte Formulierung 'Beziehung zu einem Gegenstand oder zu einem Prozeb' läßt vermuten, daß alle diese Fälle in der vorliegenden Arbeit nicht zur deadjektivischen Wortbildung zählen, da die von LOPAIIN und ULUCHANOV als ableitend aufgefaBten Adjektive nur in formaler Hinsicht als Derivateme gelten können. Inhaltlich korrelieren die in den Beispielen genannten Adjektive durchweg mit den entsprechenden Substantiven bzw. mit solchen Wortverbindungen, in denen ein Adjektiv als Komponente enthalten ist (vgl. подслизистый = Находящй̆ся под Слизистой оболочкой). Folglich haben wir es, wie schon so oft, mit Beziehungen vom Typ (8) zu tun. Adäquater wäre, diese Typen, wie in der vorliegenden Arbeit geschehen (vgl. S. 78f), nicht als präfixale deadjektivische Bildungen aufzufassen, sondern als desubstantivische präfixal-suffixale. 
1.3. Hervortreten des Merkmals in höchster oder in übermässiger Form. Bsp.: Здоровый (89) - здоровенный (2)

(D-)2. Negierung oder Nichtvorhandensein des mit dem ableitenden Adjektiv bezeichneten Merkmals oder Ausdruck der Entgegengesetztheit zu diesem. Bsp.: честный (130) - нечестный (4) моральный (66) - аморальный (8) естественный (43) - противоестественный (5)

(D-) 3. Expressive Nuancierung des Merkmals und gleichzeitiges Hervortreten desselben in verstärkter Form. Bsp.: бледный (45) - бледненький $(1)^{9}$

Bei der Beschreibung der 'deadjektivisch-verbalen Wortbildungskategorien' verfährt MANUČARJAN (1975, 346f) so, daB er, im Gegensatz zur AG-80 (I, $339 \mathrm{ff}$ ) und $z u$ der in dieser Arbeit gewählten Vorgehensweise, eine Einteilung der Verben in transitive und in intransitive vornimmt, weshalb die insgesamt sechs von inm aufgestellten 'Kategorien' teilweise rekurrent sind. Die unten angeführten allgemeinen Explikationen der Inhaltsseiten der deadjektivischen Verben stellen den Versuch dar, die Kategorien MANUČARJANs sowie die insgesamt sieben von den Autoren der $A G-80$ ermittelten 'Wortbildungsbedeutungen' auf einen gemeinsamen Nenner zu bringen, der alle Spezialfälle abdeckt.

1. Das mit dem ableitenden Adjektiv benannte Merkmal annehmen oder an den tag legen. вsp.: слепой (31) - слепнуть (1)

2. Etwas (jdn.) mit dem durch das ableitende Adjektiv benannte Merkmal versehen. Bsp.: трудный (143) - затруднить (7)

9 Bei LOPATIN Und ULUCHANOV schlieBt sich mit der 'Funktion der stilistischen Modifikation noch eine weitere 'Wortbildungsbedeutung' an (Bsp.: немудренњй - немудрячий). Ungeachtet dessen, daß die beiden in einer Relation befindlichen Adjektive jeweils unterschiedliche Stilebenen signalisieren, handelt es sich auch hier um unechte Derivationsbeziehungen, da die Inhaltsseiten der entsprechenden Lexeme im wesentlichen identisch sind. 
2.1. In geringem Маßе. Вsp.: сухой (87) - подсушить (-)

2.2. In stärkerem Мaße. Bsp.: высокий (549) - повысить (40)

2.3. Ubermäßig. Bsp.: полный (515) - переполнить (10)

3. Etwas (jdn.) des mit dem ableitenden Adjektiv benannten Merkmals berauben. Bsp.: общий (401) - разобщить (1)

Für die deadjektivischen Adverbien ${ }^{10}$ werden in der vorliegenden Arbeit die folgenden Explikationen angesetzt (vgl. AG-80, I, $409 \mathrm{f})^{11}$ :

1. Hervortreten des mit dem ableitenden Adjektiv benannten Merkmals in verstärktem Maße.

Bsp.: давний (35) - давньм (-давно) (7)

2. Uberführung von etwas/jemanden in einen Zustand, der sich durch das mit dem ableitenden Adjektiv benannte Merkmal auszeichnet. Bsp.: красный (371) - докрасна (1)

3. Gerichtetheit auf das mit dem ableitenden Adjektiv benannte Merkmal. Bsp.: Правый (204) - направо (41)

4. Seinen Anfang bei dem nehmen bzw. von dem ausgehen, was mit dem ableitenden Adjektiv benannt ist. Bsp.: высокий (549) - свысока (5)

10 Als Adverbien gelten hier die GASPAROVschen Gruppen (3) und (4); vgl.o., S. 26).

11 LOPATIN und ULUCHANOV ordnen zU Recht den Großteil der deadjektivischen Adverbien der Gruppe mit transponierender 'Wortbildungsbedeutung' zu. Von diesen Adverbien wird gesagt, sie vereinigten in ihrer Bedeutung die dem ableitenden Wort eignende 'Merkmalsbedeutung' mit der 'Bedeutung des Adverbs als Redeteil', von der es an anderer Stelle (vgl.

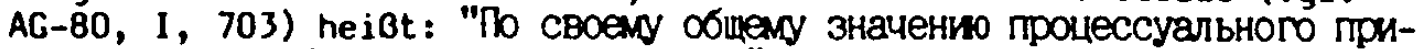
знака наречия близки прилагательными". Aus diesem Grund (Identität der nichtgrammatischen Bedeutung des Adjektivs und der des Adverbs) finden alle Adverbien mit rein transponierender Bedeutung keinen Eingang in die empirische Untersuchung. 


\subsection{Analytischer Teil}

Dem analytischen Teil dieser Arbeit ist die Beschreibung der Mittel vorbehalten, die die im vorigen Kapitel zusammengestellten nichtgrammatischen Inhaltselemente der deadjektivischen Redeteile anzeigen. Es ist anzunehmen, dab daran einerseits solche Elemente beteiligt sind, die als vom Stamm des ableitenden Adjektivs eingebracht gedacht werden können, und zum anderen solche, die die nichtgrammatische Bedeutung des jeweils ableitenden Adjektivs in bestimmter Weise modifizieren.

Im weiteren wird es also darum gehen, zunächst die bei der Derivation mit der Basisform zu verbindenden Affixe, danach die im Vergleich mit dem Derivat festzustellenden segmentphonematischen bzw. morphonologischen Veränderungen der Bezugsform sowie schließlich die akzentuellen Unterschiede zwischen Bezugsform und Derivat aufzulisten.

2.3.1. Auflistung der an der deadjektivischen Wortbildung des Russischen beteiligten Affixe

2.3.1.1. Präfixe (P)

$$
\begin{aligned}
& \text { /a/ } \\
& \text { /ant,i/ } \\
& \text { /arx,il } \\
& \text { /b,ez/ v/b,es/l2 } \\
& \text { /im/ v/ir/" } \\
& \text { /nai/ } \\
& \text { /n,e/ } \\
& \text { /n,eb,ez/ } v / n, e b, e s / \\
& \text { /p,er,e/ } \\
& \text { /po/ } \\
& \text { /pr,e/ } \\
& \text { /prot,ivo/ }
\end{aligned}
$$

12 Das ' $v$ ' steht hier und im folgenden für 'oder'.

- Diese Präfixe sind komplementär distribuiert. 


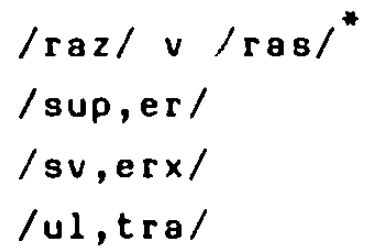

$2 \cdot 3 \cdot 1.2$. Suffixe (s)

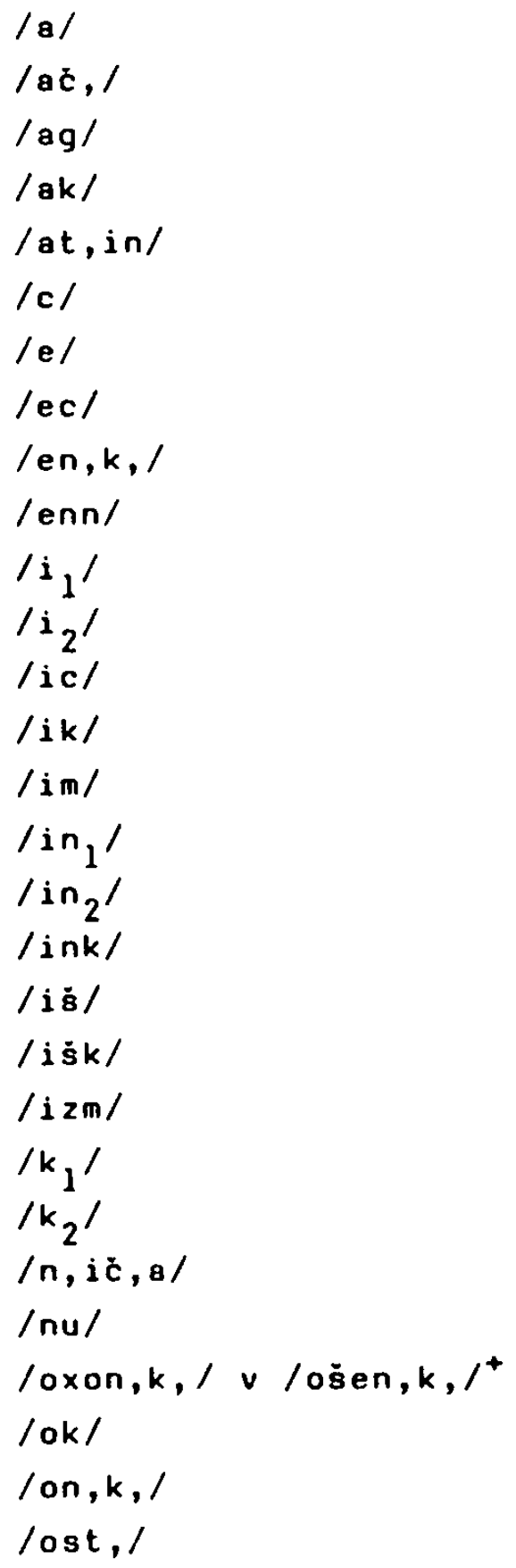

+ Diese Suffixe sind freie Varianten. 
/ot/

loval

lova $a_{2}$

lovat/ v /evat/"*

/s̆/

/ šč, in/

/stv/

/stuova/

$\mid u x /$

/ug/

/uk/

/ul, /

/us̆/

/ušč, /

/us, en, k,/

/DI

2.3.1.3. Kombinationen von Präfix und Suffix (PuS)

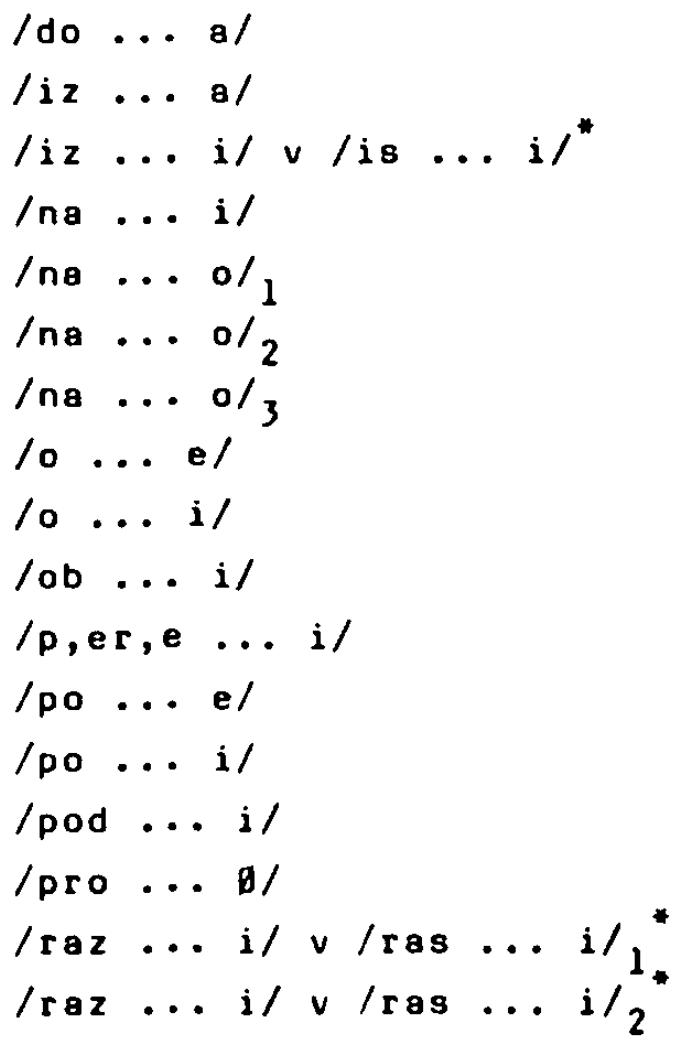

* Diese Suffixe sind komplementär distribuiert.

- Die jeweiligen Präfixe sind komplementär distribuiert. 


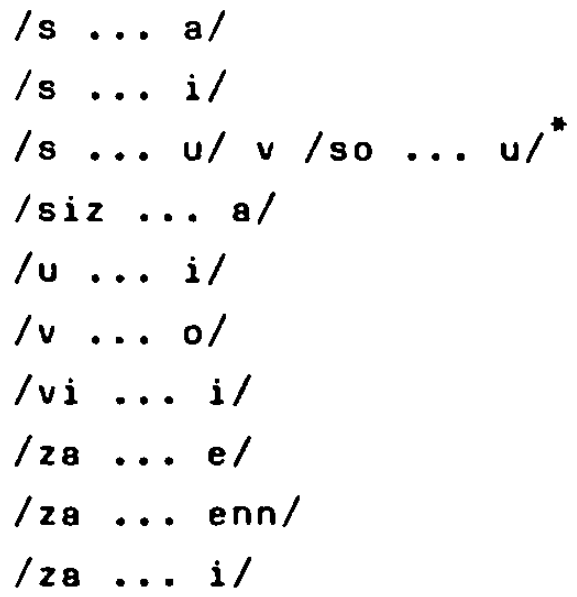

2.3.1.4. Kombinationen von Suffix und Postfix (SuPo) 13

$$
\begin{array}{lll}
/ i & \ldots & 8,8 / 1 \\
/ i & \ldots & 8,8 / 2
\end{array}
$$

2.3.2. Die morphonologischen Veränderungen der Bezugsform (M)

2.3.2.1. Verkürzungen der Bezugs form ( $\left.M_{1}\right)$

Die Bezugsform kann um die folgenden finglen Phoneme bzw. Phonemverbindungen verkürzt sein:

$$
\begin{aligned}
& M_{1.1} / a 1, n / \\
& M_{1.2 \mathrm{lan} /} \\
& M_{1.3} / \mathrm{at} / \\
& M_{1.4} / \mathrm{enn} / \\
& \text { M.5 /esk,/ } \\
& M_{1.6} / i c \text {,esk,/ } \\
& M_{1.7} / \mathrm{ic}, \mathrm{n} / \\
& \text { M.8 /irovann/ } \\
& \text { M.9/istsk,/ } \\
& M_{1.10 / \mathrm{iv} /} \\
& M_{1.11 / k, /}
\end{aligned}
$$

13 Der Begriff des 'Postfixes' blieb undefiniert (vgl.o. 1.2.2.7.). Stattdessen sollen die oben (s. S. 28) unter (3) als Derivationselemente bezeichneten Einheiten als 'Postfixe' gelten. 


$$
\begin{aligned}
& M_{1.12} / \mathrm{n} / \mathrm{v} / \mathrm{n}, / \\
& M_{1.13} / \mathrm{ok}, / \\
& M_{1.14} / \mathrm{ov} / \\
& M_{1.15} / \mathrm{r} / \\
& M_{1.16} / \mathrm{sk}, /
\end{aligned}
$$

2.3.2.2. Erweiterungen der Bezugsform $\left(\mathrm{M}_{2}\right)$

Die Bezugsform kann um folgende Phoneme bzw. Phonemverbindungen erweitert sein:

$$
\begin{aligned}
& M_{2.1} / \mathrm{aj} / \\
& \text { M2.2 lan/ }
\end{aligned}
$$

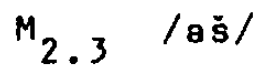

$$
\begin{aligned}
& M_{2.4} \text { /av, / }
\end{aligned}
$$

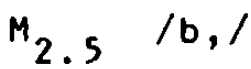

$$
\begin{aligned}
& M_{2.6 \text { /̌, / }} \\
& \mathrm{M}_{2.7} \mathrm{/e} / \\
& M_{2.8 / e j /} \\
& M_{2.9} \text { lev,l } \\
& M_{2.10} / \mathrm{in} / \\
& M_{2.11 / i r /} \\
& M_{2.12 / i z /} \\
& M_{2.13} / \mathrm{iz,ir/} \\
& M_{2.14} / 1, / \\
& M_{2.15} / \mathrm{n} / v / \mathrm{n}, / \\
& M_{2.16} \text { lov/ v lov,/ } \\
& M_{2.17 / 8 /} \\
& M_{2.18} / \mathrm{s,e/} \\
& M_{2.19} \text { /us/ }
\end{aligned}
$$


2.3.2.3. Vokalalternationen $\left(M_{3}\right)$

Beim Vergleich der Bezugsform mit dem Stamm des Derivats lassen sich die nachstehenden Vokalalternationen beobachten:

$$
\begin{aligned}
& M_{3.1} / 0 / \sim / e / \\
& M_{3.2} / 0 / \sim / 0 / \\
& M_{3.3} / \mathrm{la} / \mathrm{le} / \\
& \mathrm{M}_{3.4 \mathrm{Ia} / \sim \mathrm{IO} /} \\
& M_{3.5} / \mathrm{L} / \sim \mathrm{lol} \\
& M_{3.6} / 010 / \sim / 010 / \\
& M_{3.7} \text { /orol } / \text { gral } \\
& M_{3.8} / \text { gr,e/ } / \text { er,el }
\end{aligned}
$$

\subsubsection{Konsonantenalternationen}

Im Stamm des Derivats können (auch dann, wenn dieser zuvor im Vergleich mit dem des Derivatems verküzt wurde) die folgenden finalen konsonantischen Phoneme bzw. Phonemverbindungen der Bezugsform auf die unten beschriebenen Arten alternieren.

2.3.2.4.1. Palatalitäts- $\left(M_{4}\right)$ und Dispalatalitätsalternationen $\left(M_{5}\right)$

Parig harte Konsonanten alternieren mit den ihnen enteprechenden weichen Konsonanten und parig weiche Konsonenten mit den ihnen entsprechenden harten Konsonanten.

$$
\begin{aligned}
/ \mathrm{p} / & \sim / \mathrm{p}, / \\
/ \mathrm{b} / & \sim / \mathrm{b}, / \\
/ \mathrm{v} / & \sim / \mathrm{v}, / \\
/ \mathrm{m} / & \sim / \mathrm{m}, / \\
/ \mathrm{t} / & \sim / \mathrm{t}, / \\
/ \mathrm{d} / & \sim / \mathrm{d}, / \\
\mathrm{m} / \mathrm{s} / & \sim / \mathrm{s}, / \\
\mathrm{|z} / & \sim / \mathrm{z}, / \\
/ \mathrm{n} / & \sim / \mathrm{n}, / \\
/ \mathrm{l} / & \sim / 1, / \\
/ \mathrm{r} / & \sim / \mathrm{r}, / \\
/ \mathrm{g}, / & \sim / \mathrm{g} /
\end{aligned}
$$




$$
\begin{array}{ll}
M_{5.2} / k, / \sim / k / \\
M_{5.3} / n, / \sim / n / \\
M_{5.4} / 1, / \sim / 1 /
\end{array}
$$

\section{$2 \cdot 3 \cdot 2 \cdot 4 \cdot 2$. Transitivitätsalternationen $\left(M_{6}\right)$}

2.3.2.4.2.1. Alternationen harter oder weicher Velare mit Alveolaren

$$
\begin{aligned}
& |g / v| g, / \sim \mid \check{z} / \\
& M_{6.1}|k / \vee / k, / \sim| \check{c}, / \\
& \mid x / \vee / x, / \sim / \check{s} /
\end{aligned}
$$

2.3.2.4.2.2. Alternationen von Alveolaren mit harten Velaren

$$
\begin{array}{ll}
M_{6.2}|z / \sim| g / \\
|\check{l} / \sim| k \mid \\
|\xi| \sim|x|
\end{array}
$$

2.3.2.4.2.3. Alternationen von dentaler mit alveolarer Affrikate

$$
M_{6.3} / \mathrm{c} / \sim \text { / }, /
$$

2.3.2.4.2.4. Alternationen von alveolarer mit dentaler Affrikate

$$
M_{6.4} / \mathrm{d}, / \sim / c /
$$

2.3.2.4.2.5. Alternationen von hartem dentalem Verschlublaut mit alveolarer Affrikate

$$
M_{6.5} / \mathrm{t} / \sim / \mathrm{l}, /
$$

2.3.2.4.2.6. Alternationen von hartem dentalem mit hartem alveolarem Reibelaut

$$
M_{6.6 / 2 / \sim / z /}
$$

2.3.2.4.2.7. Alternationen von harter Liquida mit hartem velarem Reibelaut

$$
M_{6.7} / 1 / \sim / x /
$$

2.3.2.4.2.8. Kombination von hartem velarem und weichem velarem VerschluBlaut alterniert mit weichem velarem VerschluBlaut

$$
M_{6.8} / g k, / \sim / k /
$$

2.3.2.4.2.9. Kombination von hartem dentalem Reibelaut und weichem velarem VerschluBlaut alterniert mit hartem alveolarem oder mit weichem velarem Reibelaut

$M_{6.9} / \mathbf{k}, / \sim / \check{z} / v / z, /$

2.3.2.4.2.10. Kombination von hartem dentalem Reibelaut und har- 
tem dentalem Verschlublaut olterniert mit weichem alveolarem Reibelaut

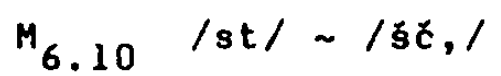

2.3.2.4.2.11. Kombination von harter Liquida mit weichem velarem Verschlublaut alterniert mit einer Kombination von weicher Liquida und alveolarer Affrikate

$M_{6,11} / 1 k, / \sim / 1, t, /$

2.3.3. Die Akzentverhältnisse (A) bei der deadjektivischen Derivation des Russischen

Die Deskription der suprasegmentalen Mittel, die gleichfalls das Vorliegen von Ableitungsrelationen signalisieren, berücksichtigt in bezug auf das Derivat lediglich die im Lexikon notierte Wortform; von den paradigmatischen Realisierungen des Lexems und somit auch von den möglichen Akzentalternationen innerhalb seines Paradigmas wird abstrahiert.

Je nachdem, ob die Betonung des Derivats mit der des ableitenden Adjektivs als korrelierend aufgefaBt werden kann oder nicht, sollen die bei der deadjektivischen Wortbildung zu beobachtenden Akzentverhältnisse in zwei Gruppen beschrieben werden:

1. Unabhängig von der Betonung des Derivatems ist beim Derivat $A_{1.1}$ das Präfix bzw. die erste Silbe akzentuiert. 14

$A_{1.2}$ die Silbe vor dem Suffix akzentuiert. ${ }^{15}$

$A_{1.3}$ das Suffix akzentuiert, und, wenn dieses mehrsilbig ist, $A_{1.3 .1}$ die erste Silbe oder

$A_{1.3 .2}$ die zweite Silbe.

14 Der Silbenbegriff bleibt hier undefiniert. Statt dessen soll angenommen werden, ein Suffix enthalte so viele Silben wie es Vokale aufweist. Der Terminus 'erste Silbe' läßt sich dann durch 'erster Vokal', der der 'zweiten Silbe' durch 'zweiter Vokal' ersetzen.

15 Die Termini 'erste Silbe' und 'präsuffixale Silbe' können eines und dasselbe bezeichnen. Daher soll für dieses Fälle (wenn dem Suffix nur eine Silbe vorausgeht und dieses betont ist) immer $A_{1.1}$ gelten. 
$A_{1.4}$ die Flexionsendung akzentuiert.

2. In Abhängigkeit von der Betonung des Derivatems ist beim Derivat

$A_{2.1}$ die gleiche Silbe wie beim ableitenden Adjektiv akzentuiert.

$A_{2.2}$ die gleiche Silbe des Stamms wie beim ableitenden Adjektiv akzentuiert, sofern letzteres (auch in seinen sogenannten Kurzformen) Stammbetonung aufweist.

$A_{2.3}$ entweder die Silbe vor dem Suffix oder

$A_{2.4}$ das Suffix akzentuiert, und, wenn dieses mehrsilbig ist, $A_{2.4 .1}$ die erste Silbe oder

$A_{2.4 .2}$ die zweite Silbe oder

$A_{2.5}$ die Flexionsendung akzentuiert, sofern das ableitende Adjektiv in seinen Lang-und/oder in seinen Kurzformen Endungsbetonung aufweist.

Zur Verdeutlichung der Beziehungen vom Typ $A_{2}$ seien einige Beispiele angeführt:
a) [umn-ij](151)-[úmstvovat, ] (1)
$\left(A_{2.1}\right)$

b) [vnútr,enn, -ij] (177) - [vnútr,ennost,i]

(6) $\left(A_{2.2}\right)$ aber

$[g n, i l-\delta j](17)-[g n, i l o s t],(1)$

c) [č,ástn-ij] (60)-[č,ástn,ik] (4)

[stár-ij] ([star-á]) (468)-[star,ik] (252)

d) $[n, e u d o ́ b n-i j](3)-[n, e u d o ́ b, e](-)$

[zil-ój] (24)-[zil,ó] (13) 


\subsection{Synthetischer Teil}

Auf rein deskriptiver Grundlage wird in möglichst übersichtlicher Form zu zeigen sein, welche Typen von Bezugsformen und welche der in 2.3.1. bis 2.3.3. oufgelisteten Ausdrucksmittel im Russischen miteinander kombiniert werden, um deadjektivische Redeteile mit den in 2.2. genannten Inhaltselementen zu bilden. Im AnschluB an die Beschreibung der tatsächlichen Kombinationen wird die Anzahl der theoretisch maximal möglichen sowie der theoretisch minimal möglichen Verbindungen dieser Mittel notiert. ${ }^{16}$ Nachdem diese Werte für den gesamten Bereich der deadjektivischen Wortbildung feststehen, ist aufgrund der Gesamtzahlen der jeweils maximalen, der minimalen und der realen Kombinationen der Wert des sogenannten Maßes der Verbundenheit' zu ermitteln, das ganzheitlich charakterisiert, in welchem Grad das Russische im Rahmen der Wortbildung Regelhaftigkeiten unterliegt, die dann schematisch aufgezeigt werden sollen.

Zunächst soll es darum gehen, die eingangs genannte Aufgabe durchzuführen, also zu beschreiben, wie bestimmte Mittel bei der deadjektivischen Derivation tatsächlich miteinander kombiniert sind und wie sie theoretisch miteinander verbunden sein können. Dabei werden, was die Deskription der realen Kombinationen anbelangt, lediglich solche Lexeme berücksichtigt, die in mindestens einem der folgenden Nachschlagewerke verzeichnet sind: BIELFELDT et al. ( $\left.{ }^{9} 1972\right)$, LEPING et al. ( $\left.{ }^{8} 1978\right)$, OŽEGOV ("1977), ZALIZNJAK (1977).

Die unten verwendeten Symbole stehen jeweils für folgendes: $\underline{D-}=$ nichtdeadjektivischer Derivatemtyp (vgl.0., 77-83), $(\underline{\text { (D) }}=$ deadjektivischer Derivatemtyp (vgl.0., 85f); $\underline{P}=$ Präfix (vgl.

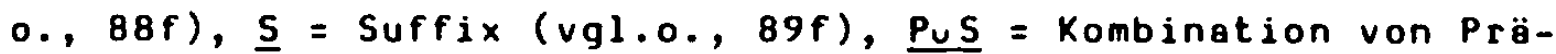

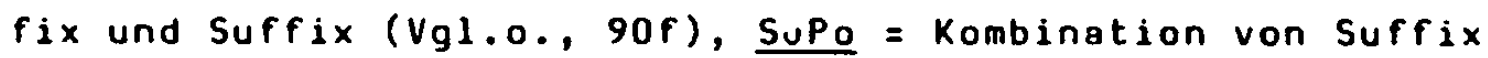
und Postfix (vgl.o., 91); $\underline{M}=$ morphonologische Veränderung der

16 Wenn hier und im folgenden von der Anzahl der Kombinationen von Mitteln die Rede ist, so sind immer ausschließlich die in 2.3.1. bis 2.3.3. angeführten Mittel gemeint. Dies bedeutet, daB die unterschiedlichen Bezugsformtypen zwar deskriptiv berücksichtigt werden, sie aber bei der Bestimung der Anzahl der Kombinationen keine Rolle spielen. 
Bezugsform (vgl.0., 91-95); $\underline{A}=$ Akzentverhältnisse (vgl.0., 95f).

Die Art des im folgenden gewählten Prozedere sei an einem Beispiel verdeutlicht: Zum Ausdruck deadjektivischer Verben mit der allgemeinen Explikation 'Etwas (jdn.) mit dem durch das ableitende Adjektiv benannte Merkmal versehen' lassen sich $u$. a. die auf $\mathrm{S}$. $147 \mathrm{f}$ unter 2.4.4.2.0.1.5. notierten Ausdrucksmittel nachweisen. Ferner sind - in vereinfachender Notation die folgenden Kombinationen dieser Mittel belegt (vgl. S. 153 unter $2.4 .4 .2 \cdot 0.2 .5$.$) :$

$$
\text { Pus/za ... } \begin{aligned}
& M_{1.1104} \\
& M_{4} \\
& M_{1.1204} \\
& M_{1.1304}
\end{aligned}
$$

Unsere empirigchen Beobachtungen ergeben demnach eine Anzahl von insgesamt vier realisierten Kombinationen. Der Umstand, daB keine anderen Kombinationen belegt sind, läbt jedoch nicht den Schlub zu, daB das Russische nicht noch weitere Kombinationen dieser Ausdrucksmittel potentiell bereithält.

Tatsächlich sind zehn weitere Kombinationen theoretisch möglich, die nun zusammengestellt und an jeweils einem, empirisch nicht belegten und daher mit (*) versehenen Beispiel veranschaulicht werden sollen.

Zunächst kann theoretisch angenommen werden, daB überhaupt keine morphonologische Alternation stattfindet. In diesem Fall ist die Konfiguration von Präfix und Suffix mit jeweils einer der beiden Ausprägungen des Akzents kombiniert:

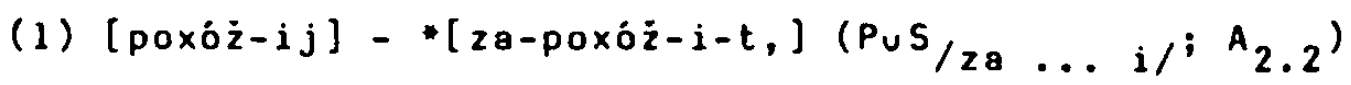

(2) [óbšč , $-i j]-*[z a-o b s ̌ c,-i-t],\left(P \cup S / z a \ldots i / A_{2.4}\right)$

Im Bereich des theoretisch Möglichen liegt ferner, daB neben die Kombination (PuS/za ... i/; M $1.11 \cup 4 ; A_{2.4}$ ) die Kombination $\left(P \cup S / z a \ldots i / M_{1.11 \cup 4} ; A_{2.2}\right.$ ) tritt: 
(3) $[j o ́ m k,-i j]-*[z a-j o ́ m,-i-t$,

Analog verhält es sich bei den Kombinationen (PuS/za ... i/; $M_{1.12 \cup 4} ; A_{2.2}$ ) sowie ( $P u S / 2 a \ldots i / M_{1.13 u 4 ;} A_{2.2}$ ) zu denen gleichfalls das andere Akzentschema treten kann:

(4) [znátn-ij]-[2a-znat,-i-t, ]

$$
\left(P \cup S / 2 a \ldots i / M_{1.12 \cup 4} ; A_{2.4}\right)
$$

(5) [glubók, -ij] - [za-glub, -i-t, ]

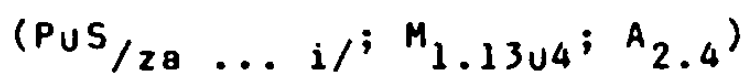

AuBerdem läBt sich denken, dab die Basisform um/k,/ bzw. um /n/ verkürzt werden kann, ohne dab zusätzlich eine Palatalitätsalternation stattfindet. (Eine Verkürzung der Basisform um /ok,/ ist dagegen nicht möglich, ohne daB der letzte Konsonant der verkürzten Bezugsform gleichzeitig gemäB der Palatalitätsalternation wechselt!)

(6) $[k a ́ c ́, k,-i j]-*[z a-k a ́ c ́,-i-t, j$

$$
\left(P \cup S / 28 \ldots i / M_{1.11} ; A_{2.2}\right)
$$

(7) $[g o r, k,-i j]-*[z 8-g o r,-i-t$,

$$
\left(P \cup S / z a \ldots i / M_{1.11} ; A_{2.4}\right)^{17}
$$

17 Eine durchaus mögliche, sogar 'wahrscheinlichere' Kombination wäre hier (PuS/za ... i/ $; M_{5 u 6.1} ; A_{2.4}$ ) (Vgl. etwa [0-gorć, $\left.-i-t,\right]$ ). Dennoch bleiben solche Müglichkeiten aus zwei Gründen bewuBt außer Betracht.

(1) Wirde man als Grundlage flur die Zusammenstellung der theoret isch möglichen Konfigurationen nicht die Gesamtheit der jeweils tatsächlich ausgenutzen Mittel (in unserem Beispiel die Gesamtheit der unter 2.4.4.2.0.1.5. notierten Ausdrucksmittel), sondern die Gesamtheit aller Ausdrucksmittel wählen, über die das Russische im Rahmen der deadjektivischen Wortbildung (und sei es auch nur der Verben) verfügt, wäre eine immens hohe Zahl theoretisch möglicher Kombinationen die Folge. Dies würde vermutlich zum einen sehr viele Irrtümer infolge einer ungewollten 'Unterschlagung' möglicher Kombinationen nach sich ziehen und wäre zum anderen nur mit übergroßem Aufwand nachzuprüfen. Daher fällt die Entscheidung zugunsten der genannten bescheideneren, doch dafür ohne weiteres überschaubaren Basis. (2) Ferner stellt sich dann, wenn man z.B. weitere morphonologische Alternationen einbezieht, die nicht in dem jeweiligen Basiskorpus enthalten sind, in vielen Fällen ohnehin keine qualitative Veränderung ein, da die Arten der Alternationen (Bezugs formuerkürzung oder -erweiterung, Palatalitäts- oder Transitivitätsalternation usw.) häufig die gleichen bleiben und sich so lediglich die Anzahl ihrer jeweiligen Ausprägungen erhöht, was die unter (1) beschriebenen Nachteile mit sich bringt. 


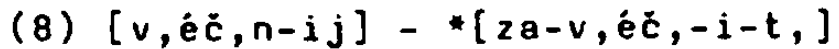

$$
\left(P \cup S / z a \ldots i / M_{1.12} ; A_{2.2}\right)
$$

(9) $[m r a ́ c ̌, n-i j]-*[z a-m r a c,-i-t$,

$$
\left(P \cup S / z a \ldots i / M_{1.12} ; A_{2.4}\right)
$$

SchlieBlich liegt es noch im Bereich des Möglichen, daB neben die Kombination ( $P \cup S / z a \ldots i / M_{4} ; A_{2.4}$ ) theoretisch die Kombination ( $\mathrm{PuS} / \mathrm{za} \ldots \mathrm{i} / \mathrm{M}_{4} ; \mathrm{A}_{2.2}$ ) tritt:

(10) [kras,iv-ij]-*[za-kras,iv,-i-t,]

Die Anzahl der theoretisch minimal möglichen Kombinationen läBt sich feststellen, indem man überprüft, ob eine der tatsächlich realisierten Kombinationen entfallen kann, ohne dab sich der zuvor jeweils empirisch belegte Bestand an Ausdrucksmitteln verringert. In unserem Beispiel ist die Kombination $\left(P u S / z a \ldots i / M_{4} ; A_{2.4}\right.$ ) 'entbehrlich', da alle in ihr enthaltenen Ausdrucksmittel auch Bestandteile einer anderen realisierten Kombination sind (vgl. ( $\left.P u S / z a \ldots i / M_{1.1144 ;} A_{2.4}\right)$ ). Die Anzahl der theoretisch minimal möglichen Kombinationen beträgt hier demnach drei.

2.4.1. Deadjektivische Substantive mit der Bedeutung eines abstrakten Merkmals

2.4.1.1. Merkmal als gesellschaftliche, politische, wissenschaftliche oder ästhetische Erscheinung oder Tendenz

2.4.1.1.1. Der Derivatemtyp und die Kombinatorik der Mittel zur Bildung von Substantiven mit dieser allgemeinen Explikation

$\left(\mathrm{S} / \mathrm{izm} / \mathrm{M}_{1.6} ; \mathrm{A}_{1.3}\right)_{\mathrm{u}}(\mathrm{D}-\mathrm{II} / \mathrm{l})$

$[k u b, i c, e s k,-i j](16)-[k u b,-1 z m](-)$

$\left(\mathrm{S} / \mathrm{izm} / \mathrm{M}_{1.12 \cup 4} ; A_{1.3}\right) u(\mathrm{D}-\mathrm{II} / 1)$

[gumánn-ij] (12)-[guman,-izm] (-)

$\left(\mathrm{s} / \mathrm{izm} / \mathrm{M}_{1.16 u 4} ; A_{1.3}\right) \cup(D-11 / 1)$

[r,espubl,ikánsk, -ij] (37) -

[r,espubl, ikan, $-12 \mathrm{~m}]$

$\left(\mathrm{S} / \mathrm{izm} / ; \mathrm{M}_{4} ; A_{1.3}\right) \cup(D-I I / 1)$ 


$$
\text { [bitov-ój] (38)-[bitov, -12m](-) }
$$

Anzahl der tatsächlich realisierten Kombinationen: 4

Anzahl der theoretisch maximal möglichen Kombinationen: ?

- Zusätzlich zu den realisierten sind die Kombinationen $\left(\mathrm{S} / \mathrm{izm} / \mathrm{A}_{1.3}\right),\left(\mathrm{S} / \mathrm{izm} / \mathrm{M}_{1.12} ; \mathrm{A}_{1.3}\right)$, $\left(\mathrm{S} / \mathrm{izm} / ; M_{1.16} ; A_{1.3}\right)$ theoretisch möglich.

Anzahl der theoretisch minimal möglichen Kombinationen: $\underline{3}$

- Theoretisch möglich wäre ein Wegfall der Kombination $\left(\mathrm{S} / \mathrm{izm} / \mathrm{M}_{4} ; A_{1.3}\right)$.

2.4.1.2. Erscheinung der auBersprachlichen Wirklichkeit, die charakterisiert ist durch das Vorliegen des mit dem ableitenden Adjektiv benannten Merkmals in abgeschwächter Form

2.4.1.2.1. Aufzählung der Derivatemtypen und der jeweils miteinander kompatiblen Mittel zur Bildung von Substantiven mit dieser allgemeinen Explikation

$2.4 .1 .2 .1 .1, D-I, D-I I / 1, D-V / 4$

$$
\begin{aligned}
& S / \text { in } / \\
& M_{4}, M_{6.1} \\
& A_{2.2}, A_{2.5}
\end{aligned}
$$

$2.4 .1 .2 .1 .2, D-1, D-11 / 1, D-I I / 2, D-I I / 12, D-V / 5,(D-) 1.1$

S/ink/

$M_{1.12}$ (ачвег bеi краснинка, скучнинка), $M_{4}$,

$M_{6.1}$

$$
A_{2.2}, A_{2.4}
$$

$2.4 \cdot 1.2 \cdot 1.3 . D-1, D-I I / 1$

$\mathrm{S} / \mathrm{c} /$

M1.12 (веі прохладцв, ехидца),

$M_{2.7}$ (sofern die Bezugsform auf KK endet),

$M_{4}$ (wenn 2.7 , sonst $/ 1 / \sim / 1, /$ )

$A_{2.2}, A_{2.5}$ 
$2.4 \cdot 1.2 .1 .4 . \quad 0-1$

$$
\begin{aligned}
& \text { Pus/pro ... } D / \\
& M_{1.11}, M_{1.12} \text { (beide nach } K \text { ), } M_{1.13}, M_{4}, M_{6.1} \\
& A_{1.1}
\end{aligned}
$$

2.4.1.2.2. Die Kombinatorik dieser Mittel

$$
\begin{aligned}
& 2.4 .1 .2 .2 .1 . \quad\left(\mathrm{S} / \mathrm{in}_{1} / \mathrm{M}_{4} ; \mathrm{A}_{2.5}\right) u(\mathrm{D}-\mathrm{I}) \\
& {[p, \text { ostr-ij] (39) }-[p, \text { ostr,-in-á }](-)} \\
& \left(\mathrm{S} / \text { in }_{1} / \mathrm{M}_{4} ; \mathrm{A}_{2.2}\right) \cup(\mathrm{D}-\mathrm{II} / \mathrm{l}) \\
& \text { [sutúl-ij] (6) - [sutúl, -in-a] (-) } \\
& \left(\mathrm{S} / \mathrm{in}_{1} / M_{6.1} ; A_{2.2}\right) u(D-V / 4) \\
& \text { [kosobók,-ij] (1)-[kosobóc, -in-a] (-) }
\end{aligned}
$$

Anzahl der tatsächlich realisierten Kombinationen: 3

Anzahl der theoretisch maximal möglichen Kombinationen: 6

- Zusätzlich zu den realisierten sind die Kombinationen $\left(\mathrm{S} / \mathrm{in}_{1} / \mathrm{A}_{2.2}\right),\left(\mathrm{S} / \mathrm{in}_{1} / \mathrm{A}_{2.5}\right),\left(\mathrm{S} / \mathrm{in}_{1} / \mathrm{M}_{6.1} ; \mathrm{A}_{2.5}\right)$

theoretisch möglich.

Anzahl der theoretisch minimal möglichen Kombinationen: $\underline{2}$

- Theoretisch möglich wäre ein Wegfall der Kombination $\left(\mathrm{S} / \mathrm{in}_{1} / ; \mathrm{M}_{4} ; \mathrm{A}_{2.2}\right)$

$$
\begin{aligned}
& 2.4 .1 .2 .2 .2 . \quad\left(5 / \text { ink } / A_{2.2}\right) u(D-V / 5) \\
& \text { [sumass̆ć,édš-ij] (44)- } \\
& \text { [sumass̆c ,éds̆-ink-a] (-) } \\
& \left(\mathrm{S} / \mathrm{ink} / \mathrm{M}_{4} ; A_{2.2}\right) \cup(D-I I / 2) \\
& {[f a l, \check{s} i v-i j](10)-[f a l, \grave{s} i v,-i n k-a](-)} \\
& \left(\mathrm{s} / \mathrm{ink} / ; M_{4} ; A_{2.2}\right) \cup(D-11 / 12) \\
& \text { [ść, erbát-ij] (2) - [šć, erbát, -ink-a] (-) } \\
& \left(\mathrm{S} / \text { ink } / ; \mathrm{M}_{4} ; \mathrm{A}_{2.2}\right) u\left(\left(D_{-}\right) 1.1\right) \\
& \text { [grubovát-ij] (3) - [grubovát, -ink-a] (-) } \\
& \left(\mathrm{S} / \mathrm{ink} / \mathrm{M}_{4} ; \mathrm{A}_{2.4}\right) \cup(D-1 \mathrm{I} / 1) \\
& \text { [ogn,ev-ój] (6) - [ogn,ev,-ink-a] (-) } \\
& \left(\mathrm{s} / \mathrm{ink} / ; \mathrm{M}_{6.1} ; \mathrm{A}_{2.4}\right) u(D-1) \\
& {[g l u x-o j](54)-[g l u s ̌-i n k-8](-)}
\end{aligned}
$$




$$
\begin{aligned}
& \left(S / \text { ink } / M_{1.12 \cup 4} ; A_{2.4}\right) \cup(D-I) \\
& \text { [grustn-ij] (36) }-[\text { grust, -ink-a] (-) }
\end{aligned}
$$

Anzahl der tatsächlich realisierten Kombinationen: $\underline{5}$

Anzahl der theoretisch maximal möqlichen Kombinationen: 10

- Zusätzlich zu den realisierten sind die Kombinationen $\left(S_{\text {ink } /} ; A_{2.4}\right),\left(S_{/ i n k} ; M_{6.1} ; A_{2.2}\right)\left(S_{/ i n k} ; M_{1.12} ; A_{2.2}\right)$, $\left(\mathrm{S} /\right.$ ink $\left./ \mathrm{M}_{1.12} ; \mathrm{A}_{2.4}\right),\left(\mathrm{S}_{\text {/ink }} ; \mathrm{M}_{1.12 \cup 4} ; A_{2.2}\right)$ theoretisch möglich.

Anzahl der theoretisch minimal möglichen Kombinationen: $\underline{3}$

- Theoretisch möglich ist ein Wegfall der Kombinationen $\left(S_{\text {ink } /} ; A_{2.2}\right)$, (S/ink $\left./ M_{4} ; A_{2.4}\right)$.

$$
\begin{aligned}
& 2.4 .1 .2 .2 .3 . \quad\left(S / c / ; M_{1.12} ; A_{2.2}\right) \cup(D-I I / 1) \\
& {[\text { proxládn-ij] (16)-[proxlád-c-a] (-) }} \\
& \left(\mathrm{S} / \mathrm{c} / ; \mathrm{M}_{4} ; \mathrm{A}_{2.5}\right) \cup(D-I) \\
& {[g n, i 1-o j](17)-[g n, i l,-c-\dot{B}](-)} \\
& \left(\mathrm{S} / \mathrm{c} / \mathrm{H}_{\left.2.7 \cup 4 ; A_{2.5}\right) u(D-I)}\right. \\
& \text { [krásn-ij] (37i) - [krasn,e-c-á] (-) }
\end{aligned}
$$

Anzahl der tatsächlich realisierten Kombinationen: $\underline{3}$

Anzahl der theoretisch maximal möglichen Kombinationen: 10

- Zusätzlich zu den realisierten sind die Kombinationen $\left(\mathrm{S} / \mathrm{c} / ; \mathrm{A}_{2.2}\right),\left(\mathrm{S} / \mathrm{c} / ; \mathrm{A}_{2.5}\right),\left(\mathrm{S} / \mathrm{c} / ; \mathrm{M}_{1.12} ; \mathrm{A}_{2.5}\right)$, $\left(\mathrm{S} / \mathrm{c} / ; \mathrm{M}_{4} ; \mathrm{A}_{2.2}\right),\left(\mathrm{S} / \mathrm{c} / \mathrm{M}_{2.7} ; \mathrm{A}_{2.2}\right),\left(\mathrm{S} / \mathrm{c} / ; \mathrm{M}_{2.7} ; \mathrm{A}_{2.5}\right)$, $\left(\mathrm{S} / \mathrm{c} / \mathrm{M}_{2.744} ; \mathrm{A}_{2.2}\right)$ theoretisch möglich.

Anzahl der theoretisch minimal möqlichen Kombinationen: $\underline{\mathbf{2}}$

- Theoretisch möglich ist ein Wegfall der Kombination $\left(\mathrm{S} / \mathrm{c} / ; \mathrm{M}_{4} ; \mathrm{A}_{2.5}\right)$.

$$
\begin{aligned}
& \left.2.4 .1 .2 .2 .4 \text { (PuS/pro ...g/; } M_{6.1} ; A_{1.1}\right) \cup(D-I) \\
& {[t, i x,-i j](120)-[p r b-t, i b-b](-)} \\
& \text { (Pus/pro .... ; M } \left.1.11 \cup 4 ; A_{1.1}\right) \cup(D-I) \\
& {[r, e ́ d k,-i j](80)-[p r o ́-r, e d,-D](-)} \\
& \text { (Pus/pro ...g/; } M_{\left.1.12 \cup 4 ; A_{1.1}\right) \cup(D-1)} \\
& {[t, o m n-i j](223)-[p r o ́-t, o m,-D](-)} \\
& \text { (PuS/pro ... g/; M1.13u4; } A_{1.1} \text { ) } \cup(D-I) \\
& \text { [visók, -ij] (549)-[pró-vis, - B] (-) }
\end{aligned}
$$

Anzahl der tatsächlich realisierten Kombinationen: 4 
Anzahl der theoretisch maximal möglichen Kombinationen: 9

- Zusätzlich zu den realisierten sind die Kombinationen (PuS/pro ... $/$; $_{1.1}$ ), (PuS/pro ... g/; $M_{4} ; A_{1.1}$ ), (PuS/pro ... o/; $M_{1.11} ; A_{1.1}$ ), (PuS/pro ...g/; $M_{1.12}$; $\left.A_{1.1}\right),\left(P u S / p r o \ldots g / M_{1.13} ; A_{1.1}\right)$ theoretisch möglich.

Anzahl der theoretisch minimal möqlichen Kombinationen: 4

- Weniger Kombinationen als die tatsächlich realisierten sind theoretisch nicht möglich.

Zur Bildung von Substantiven mit der vorstehenden allgemeinen Explikation realisiert das Russische demnach

$$
3+5+3+4=\underline{15}
$$

unterschiedliche Kombinationen der zur Verfügung stehenden Mittel.

Theoretisch maximal möglich sind

$$
6+10+10+9=\underline{35}
$$

unterschiedliche Kombinationen dieser Mittel, und theoretisch minimal möglich sind

$$
2+3+2+4=\underline{11}
$$

unterschiedliche Kombinationen.

2.4.1.3. Merkmal als negativ bewertete Erscheinung oder Tendenz

2.4.1.3.1. Der Derivatemtyp und die Kombinatorik der Mittel zur Bildung von Substantiven mit dieser gllgemeinen Explikation

$$
\begin{aligned}
& \left(S / 8 c, \text { in/; } M_{1.12} ; A_{2.1}\right) \cup(D-I I / 1) \\
& \text { [of,iciál,n-ij] (67) - [of,icial,-gk,in-8] (-) } \\
& \left(\mathrm{S} / \text { šc̆, in/; } M_{1.16} ; A_{2.1}\right) \cup(D-I I / 1) \\
& \text { [tarabársk,-ij] (-) - [tarabár-šč,in-a] (-) }
\end{aligned}
$$

Anzahl der tatsächlich realisierten Kombinationen: $\underline{2}$

Anzahl der theoretisch maximal möglichen Kombinationen: 3

- Zusätzlich zu den realisierten ist die Kombination $\left(S / \check{s c c, i n / ;} A_{2,1}\right)$ theoretisch möglich.

Anzahl der theoretisch minimal möglichen Kombinationen: $\underline{2}$

- Weniger Kombinationen als die tatsächlich realisierten sind theoretisch nicht möglich. 
Somit verwirklicht das Russische zur Bildung von Substantiven mit der Bedeutung eines abstrakten Merkmals

$$
4+15+2=\underline{21}
$$

unterschiedliche Kombinationen der zur Verfügung stehenden Mittel.

Theoretisch maximal möglich sind

$$
7+35+3=45
$$

unterschiedliche Kombinationen dieser Mittel, und

theoretisch minimal möqlich sind

$$
3+11+2=\underline{16}
$$

unterschiedliche Kombinationen.

2.4.2. Deadjektivische Substantive mit der Bedeutung des Merkmalsträgers

2.4.2.1. Gesamtheit dessen, was sich durch ein bestimmtes Merkmal auszeichnet

2.4.2.1.1. Aufzählung der Derivatemtypen und der jeweils miteinander kompatiblen Mittel zur Bildung von Substantiven mit dieser allgemeinen Explikation

$2.4 .2 .1 .1 .1 .0-1, D-111 / 7$

S/at, in/

$\mathrm{M}_{4}$

$A_{1.3 .1}$

$2.4 .2 .1 .1 .2 .0-1, D-I I I / 1, D-I I I / 7$

s/ost, /

$\mathrm{M}_{5.3}$

$A_{2.2}, A_{2.3}$

$2 \cdot 4 \cdot 2 \cdot 1 \cdot 1.3 . \quad 0-1$

S/ot/

$M_{5.2}$

$A_{1.4}$

$2.4 .2 \cdot 1.1 .4 .0-11 / 1$ 


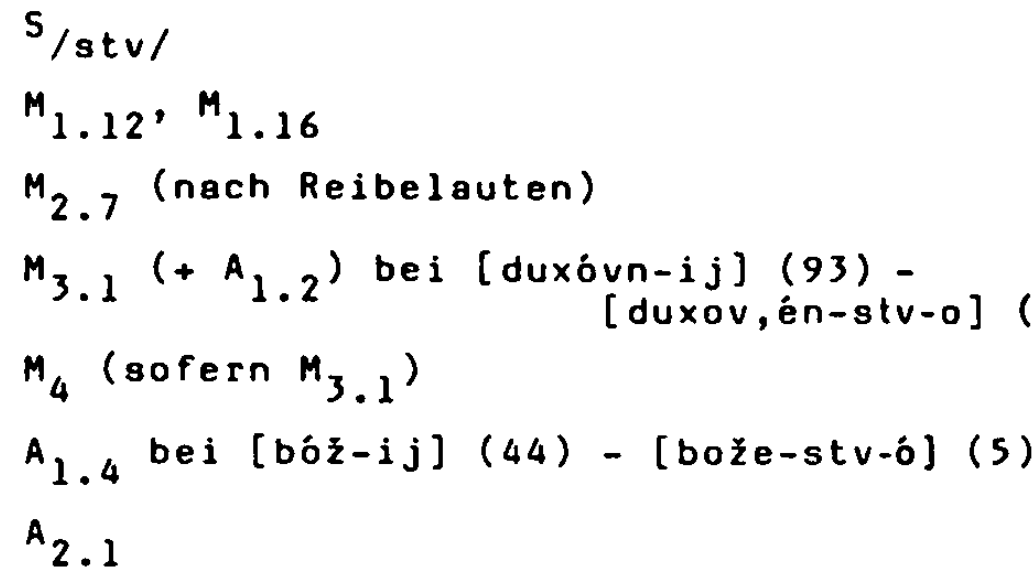

$2 \cdot 4 \cdot 2 \cdot 1.1 .5 \cdot(1$.

$D-1, D-111 / 7,(D-) 2$

$S / 0 /$

$M_{3.1}$ bei [gór,k,-ij] (43)-[gór,eí,-ø] (18)

$M_{3.2}$ bei $[m, \dot{e} 1 k,-i j](201)-[m, \dot{e} l(c ̌,-D](34)$

$M_{4}, M_{6.1}$

${ }^{1}, 1$

und (2.)

$D-I, D-I I I / 7, D-V / 4$

$s / 0 /$

$M_{1.11}, M_{1.12} ; M_{4}$

$A_{1.4}$

$A_{2.1}$ (sofern der Stamm des Derivatims drei und
mehr Silben aufweist)

$2.4 .2 .1 .1 .6 . D-I, D-I I / 1,(D-) 1.3$

$\mathrm{M}_{\mathrm{S}}$

$A_{2.1}$

2.4.2.1.2. Die Kombinatorik dieser Mittel

$$
\begin{aligned}
& 2.4 .2 .1 .2 .1 . \quad\left(\mathrm{S} / \mathrm{at}, \mathrm{in} / \mathrm{M}_{4} ; \mathrm{A}_{1.3 .1}\right) u(D-I) \\
& {[k, 181-i j](13)-[k, i s 1,-a ́ t, i n \cdot a] \text { (2) }} \\
& \text { (S/at,in/; } \left.M_{4} ; A_{1.3 .1}\right) \cup(D-1 I I / 7) \\
& \text { [túxl-ij] (1) - [tuxl, -at, in-a] (-) }
\end{aligned}
$$

Anzahl der tatsächlich realisierten Kombinationin: 1 
Anzahl der theoretisch maximal möglichen Kombinationen: $\underline{2}$

- Zusätzlich zu der realisierten ist die Kombination ( $/$ /at,in/; $\left.A_{1.3 .1}\right)$ theoretisch möglich.

Anzahl der theoretisch minimal möglichen Kombinationen: 1

$2.4 .2 .1 .2 .2 . \quad\left(5 / 0 s t, / ; M_{5.3} ; A_{2.2}\right) u(D-1)$

$$
\begin{aligned}
& \text { [vnútr,enn, -ij] (177) - } \\
& \text { [vnútr,enn-ost, -i] (6) } \\
& \text { (s/ost, } \left.; A_{2.2}\right) \cup(D-1 I 1 / 1) \\
& \text { [rast, it,el,n-ij] (33) - } \\
& \text { [rast, it,el,n-ost, ] } \\
& \left(5 / 08 t, / ; A_{2.3}\right) \cup(D-I I I / 7) \\
& {[g n, i l-o j](17)-[g n, i l-o s t,]}
\end{aligned}
$$

Anzahl der tatsächlich realisierten Kombinationen: 3

Anzahl der theoretisch maximal möglichen Kombinationen: 4

- Zusätzlich zu den realisierten Kombinationen ist die Kombination ( $\mathrm{S} / 0 \mathrm{st,f} ; \mathrm{M}_{5.3} ; \mathrm{A}_{2.3}$ ) theoretisch möglich.

Anzahl der theoretisch minimal möqlichen Kombinationen: $\underline{2}$

- Theoretisch möglich ist ein Wegfall der Kombination $\left(\mathrm{s} /\right.$ ost, $\left.; \mathrm{A}_{2.2}\right)$.

$2.4 .2 .1 .2 .3 . \quad\left(5 / 0 t / ; A_{1.4}\right) \cup(D-1)$

$$
\begin{aligned}
& {[b, e ́ d n-i j](112)-[b, e d n-o t-a ́)} \\
& \left(s / o t / ; M_{5.2} ; A_{1.4}\right) u(D-I) \\
& {[m, e ́ 1 k,-i j](201)-[m, e l k-o t-a]}
\end{aligned}
$$

Anzahl der tatsächlich realisierten Kombinationen: $\underline{2}$ Anzahl der theoretisch maximal möglichen Kombinationen: $\underline{2}$ Anzahl der theoretisch minimal möglichen Kombinationen: 1

- Theoretisch möglich ist ein Wegfall der Kombination $\left(s_{/ o t /} ; A_{1.4}\right)$.

$2.4 .2 .1 .2 .4 . \quad\left(g_{s t v} ; M_{1.12} ; A_{2.1}\right) u(D-I I / 1)$

[paroxódn-ij] (3) - [paroxód-stv-o] (6)

$\left(S / 8 t v / ; M_{1.16} ; A_{2.1}\right) u(D-11 / 1)$

[vóinsk,-ij] $(28)-[v o ́ i n-s t v-0]$

$\left(\mathrm{S} / \mathrm{stv} / ; \mathrm{M}_{2.7} ; \mathrm{A}_{1.4}\right) \cup(\mathrm{D}-\mathrm{II} / \mathrm{l})$

[bóz-ij] (44)-[bože-stv-ó] 


$$
\text { (S/stu/; M } \left.3.104 ; A_{1.2}\right) u(D-11 / 1)
$$

[duxóvn-ij] (93) - [duxov,én-stv-o] (19)

Anzahl der tatsächlich realisierten Kombinationen: 4

Anzahl der theoretisch maximal möglichen Kombinationen: 12

- Zusätzlich zu den realisierten sind die Kombinationen
$\left(\mathrm{S} / \mathrm{stv} / \mathrm{M}_{1.12} ; \mathrm{A}_{1.2}\right)$, (S/stv/; $\left.\mathrm{M}_{1.12} ; \mathrm{A}_{1.4}\right)$, (S/stv/;
$\left.M_{1.16} ; A_{1.2}\right),\left(\mathrm{S} / \mathrm{stv} / ; M_{1.16} ; A_{1.4}\right),\left(\mathrm{S} / \mathrm{stv} / M_{2.7} ; A_{1.2}\right)$,
$\left(\mathrm{S} / \mathrm{stv} / ; \mathrm{M}_{2.7} ; \mathrm{A}_{2.1}\right),\left(\mathrm{S} / \mathrm{stv} / \mathrm{M}_{3.1} ; \mathrm{A}_{1.4}\right),(\mathrm{S} / \mathrm{stv} /{ }$
$M_{3.144}$; $A_{2.1}$ ) theoretisch möglich. Noch weitere Kombina-
tionen sind zwar denkbar, nicht jedoch auch möglich.

Anzahl der theoretisch minimal möglichen Kombinationen: $\underline{4}$

$2 \cdot 4 \cdot 2 \cdot 1 \cdot 2 \cdot 5 \cdot(1$.

$$
\begin{aligned}
& \left(S / \varnothing / ; M_{3.1 \cup 6.1} ; A_{1.1}\right) \cup(D-1) \\
& \text { [gór, } k,-i j](43)-[g o ́ r, e c,-D] \text { (18) } \\
& \left(S / \emptyset / M_{3.2 \cup 6.1} ; A_{1.1}\right) \cup(D-I) \\
& {[m, \dot{e} 1 k,-i j](201)-[m, e ́ l o c ́,-\emptyset]} \\
& \left(s / g / ; M_{4} ; A_{1.1}\right) u(D-111 / 7) \\
& {[g n, i 1-6 j](17)-[g n, i 1,-\emptyset](1)} \\
& \left(S / \varnothing / ; M_{4} ; A_{1.1}\right) \cup((D-) 2) \\
& {[n, e x, 18 t-i j](13)-[n, e ́ c ̌, i s t,-B]} \\
& \left(S / \theta / M_{6.1} ; A_{1.1}\right) \cup(D-1) \\
& {[d, i k,-i j](61)-[d, i \varepsilon,-0](20)}
\end{aligned}
$$

Anzahl der tatsächlich realisierten Kombinationen: $\underline{4}$

Anzahl der theoretisch maximal möglichen Kombinationen: $\underline{7}$

- Zusätzlich zu den realisierten sind die Kombinationen

$\left(S / \varnothing / ; M_{3 \cup 4} ; A_{1.1}\right),\left(S / 0 / ; M_{3} ; A_{1.1}\right),\left(S / 0 / A_{1.1}\right)$ theoretisch möglich.

Anzahl der theoretiach minimal möglichen Kombinationen: 4

$2 \cdot 4 \cdot 2 \cdot 1 \cdot 2 \cdot 5 \cdot(2$.

$$
\begin{aligned}
& \left(S / 0 / ; M_{4} ; A_{1.4}\right) u(D-1) \\
& {[\text { stár-ij] (468)-[star,-g-ó (4) }} \\
& \text { (S/ø/; } M_{\left.1.11 \cup 4 ; A_{1.4}\right) \cup(D-1)} \\
& \text { [korótk,-ij] (9i)-[korot, - }- \text {-o] (-) } \\
& \left(\mathrm{S} / g / \mathrm{M}_{4} ; \mathrm{A}_{1.4}\right) \cup(\mathrm{D}-\mathrm{III/7}) \\
& {[g n, i l-\delta j](17)-[g n, i l,-g-\delta](1)}
\end{aligned}
$$




$$
\begin{aligned}
& \left(S / g / ; M_{\left.1.12 u 4 ; A_{2.1}\right) u(D-V / 4)}\right. \\
& \text { [povs,edn,évn-ij] (20)- } \\
& {[\text { pov8,edn,év, -g-e] (-) }}
\end{aligned}
$$

Anzahl der tatsächlich realisierten Kombinationen: $\underline{3}$

Anzahl der theoretisch maximal möglichen Kombinationen: 11

- Zusätzlich zu den realisierten sind die Kombinationen $\left.\left(S / \emptyset / ; A_{1.4}\right),\left(S / \emptyset / A_{2.1}\right),(S / \varnothing) ; M_{1.11 \cup 4} ; A_{2.1}\right),(S / \emptyset / ;$
$\left.M_{1.11} ; A_{2.1}\right),\left(S / \emptyset / M_{1.11} ; A_{1.4}\right),\left(S / \emptyset / ; M_{1.12 \cup 4} ; A_{1.4}\right)$,
$\left(S / \emptyset / ; M_{1.12} ; A_{1.4}\right),\left(S / \emptyset / M_{1.12} ; A_{2.1}\right)$ theoretisch mög-
lich.

Anzahl der theoretisch minimal möqlichen Kombinationen: $\underline{2}$

- Theoretisch möglich ist ein Wegfall der Kombination $\left(s / 0 / M_{4} ; A_{1.4}\right)$.

$$
\begin{aligned}
& : 2.4 \cdot 2 \cdot 1.2 \cdot 6 . \quad\left(A_{2.1}\right) \cup(D-I) \\
& \text { [nóv-ij] (1722) - [nóvoe] (22) } \\
& \left(M_{5} ; A_{2.1}\right) \cup(D-1 I / 1) \\
& \text { [trag,ic,esk, -ij] (15)-[trag,ic,eskoe] (-) } \\
& \left(A_{2.1}\right) \cup((D-) 1.3) \\
& \text { [pr,ekrásn-ij] (138) - [pr,ekrásnoe] }
\end{aligned}
$$

Anzahl der tatgächlich realisierten Kombinationen: $\underline{2}$

Anzahl der theoretisch maximal möqlichen Kombinationen: $\underline{2}$

Anzahl der theoretisch minimal möqlichen Kombinationen: 1

- Theoretisch möglich ist ein Wegfall der Kombination

$$
\left(A_{2,1}\right)(\ldots) \text {. }
$$

ZZur Bildung von Substantiven mit der vorstehenden allgemeiinen Explikation ('Gesamtheit dessen, was sich durch ein beestimmtes Merkmal auszeichnet') verwirklicht des Russische

$$
1+3+2+4+(4+3)+2=\underline{19}
$$

Lunterschiedliche Kombinationen der zur Verfügung stehenden A Ausdrucksmittel.

Theoretisch maximal möglich sind

$$
2+4+2+12+(7+11)+2=\underline{40}
$$

cunterschiedliche Kombinationen dieser Mittel, und theoretisch minimal möglich sind

$$
1+2+1+4+(4+2)+1=\underline{15}
$$

cunterschiedliche Kombinationen 
2.4.2.2. Jemand (etwas), der (das) sich durch ein bestimmtes Merkmal auszeichnet

2.4.2.2.1. Aufzählung der Derivatemtypen und der jeweils miteinander kompatiblen Mittel zur Bildung von Substantiven mit dieser allgemeinen Explikation ${ }^{18}$

$2.4 \cdot 2 \cdot 2 \cdot 1.1 . \quad D-1$

$$
\begin{aligned}
& S / u k / \\
& M_{4} \\
& A_{1.3}
\end{aligned}
$$

$2.4 \cdot 2 \cdot 2 \cdot 1.2$. D-I

$$
\begin{aligned}
& S / o k / \\
& M_{4} \text { bei }[m a ́ 1-i j](224)-[m a l,-o k](1) \\
& M_{6.1} \text { bei }[d, i k,-i j](61)-[d, i c, o k](-) \\
& A_{1.3}
\end{aligned}
$$

$2.4 \cdot 2 \cdot 2 \cdot 1.3 . \quad D-I$

$$
\begin{aligned}
& S / i \measuredangle k / \\
& M_{1.11} \text { bei [korótk,-ij] (11) } \\
& A_{1.3}
\end{aligned}
$$

$2.4 \cdot 2 \cdot 2 \cdot 1.4 . \quad D-1$

$$
\begin{aligned}
& S / k_{1} / \\
& A_{2.2}, A_{2.5}
\end{aligned}
$$

$2.4 \cdot 2 \cdot 2 \cdot 1.5 . \quad 0-1$

$$
\begin{aligned}
& S / a c, / \\
& M_{1.3} \text { bei [bogát-ij] (238)-[bog-ác, ] (8) } \\
& M_{5}
\end{aligned}
$$

18 Viele der üblicherweise (z.B. in der AG-80, I, 166f) zu dieser Gruppe gerechneten Bildungen sind nicht als deadjektivisch zu betrachten, sondern als von solchen Wortverbindungen abgeleitet aufzufassen, die das entsprechende Adjektiv als Konstituente enthalten. Dies gilt insbesondere für Bildungen auf $-K u(a),-K H,-K(a),-K H(a)$ sowie gänzlich für

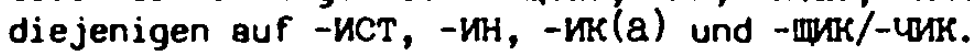




$$
A_{1.3}
$$

$2 \cdot 4 \cdot 2 \cdot 2 \cdot 1.6 \cdot D-1$

$5 / i \zeta /$

M.11

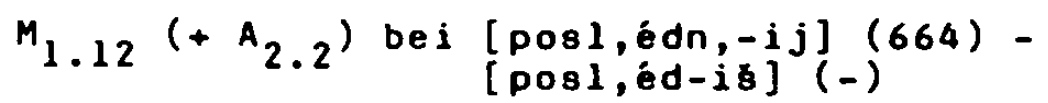

$M_{6.8}\left(+A_{2.2}\right)$ bei $[m, a ́ g k,-i j](91)-$

$A_{2.2}$ (auber in den genannten Fällen noch bei $[g l a ́ d k,-i j](26)$ -

$A_{1.3}$ sonst

$2 \cdot 4 \cdot 2.2 .1 .7$. D-I

S/ak/

$M_{1.12}$ bei [c,udn-ój] (s) - [z,ud-ák] (30)

$M_{2.6}$ bei $[8 m, e ́ 1-i j](73)-[8 m, e l, c,-8 k](6)$, $[v, e 8, o ́ l-i j](183)-$ $[v, e s, 01, \mathrm{c},-\mathbf{a k}]$ (4)

$M_{4}$ auber bei

$[c, u d n-6 j](5)-[\check{c}, u d-a ́ k](30)$ [prost-ój] (287) - [prost-ák] (7) $[\mathrm{alab}-\mathrm{ijj}](98)-[\mathrm{s}[\mathrm{ab}-\mathrm{ak}](-)$

$M_{6.1}$

$A_{1.3}$

$2.4 \cdot 2.2 .1 .8 . D-I, D-I I / 1$

S/ux/

$A_{1.3}$

2.4.2.2.1.9. D-I, D-II/I

S/U⿺辶/

$\mathrm{M}_{5}$

$A_{1.3}$

$2 \cdot 4 \cdot 2.2 \cdot 1.10 . D-I, D-I I / 1$

S/ag/

$M_{1.7}$ 


$$
\begin{aligned}
& M_{4} \\
& A_{1.3}
\end{aligned}
$$

$2 \cdot 4 \cdot 2.2 .1 .11 . D-I, D-11 / 1$

$$
\begin{aligned}
& \text { S/ul, / }
\end{aligned}
$$

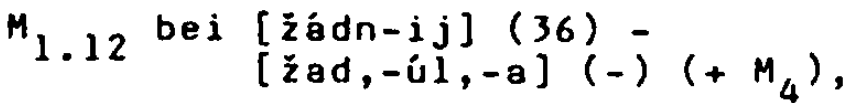

$$
\begin{aligned}
& \text { [kroxótn-ij] (32) - } \\
& \text { [kroxot-ul, -a] (-), } \\
& \text { [kapr,izn-ij] (12)'- } \\
& \text { [kapr,iz-úl,-a] (-) }
\end{aligned}
$$

$\mathrm{M}_{4}$ auber im bereits genannten fall noch bei [ ć, ist-ij] (225) -

$$
[\check{c}, \text { ist, }- \text { úl, }- \text { a }](-)
$$

$$
{ }^{A} 1.3
$$

$2.4 .2 .2 .1 .12 . D-I, D-1 I / 1$

S/ic/

$\mathrm{M}_{4}$

$M_{6.1}$

$A_{1.3}$ bei $[e d, i n-i j](87)-[e d, i n,-i c-a](36)$

$A_{2.1}$ bei [vól,n-ij] (25) $=[$ vól,n,-ic-a] (-) $[p, a n-i j](88)-[p, a ́ n,-i c-8](14)$ $[u ̛ m n-i j](151)-[u ́ m n,-i c-8](20)$

$$
A_{2.2}, A_{2.4} \text { sonst }
$$

$2 \cdot 4 \cdot 2 \cdot 2 \cdot 1 \cdot 13 . D-1, D-11 / 1$

$$
\begin{aligned}
& \mathrm{S} / \mathrm{in}_{2} / \\
& M_{1,11} \text { bei }[r, e ́ d k,-i j](80)-[r, e d,-i n-a](-) \\
& M_{1.12}
\end{aligned}
$$

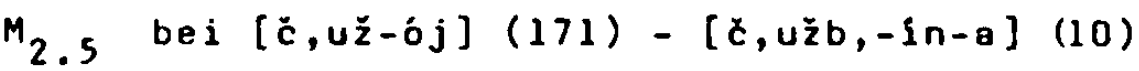

$$
\begin{aligned}
& M_{2.16} \text { bei }\left\{\begin{array}{l}
{[d, i k,-i j\}(61)-} \\
{[d, i k o b,-i n-a](7)\left(+M_{5}+A_{1.2}\right)}
\end{array}\right. \\
& [k], \dot{e} j k,-i j](5)- \\
& {\left[k 1, \text { ejkov, -in-a] (3) }\left(+A_{1,3}\right)\right.}
\end{aligned}
$$




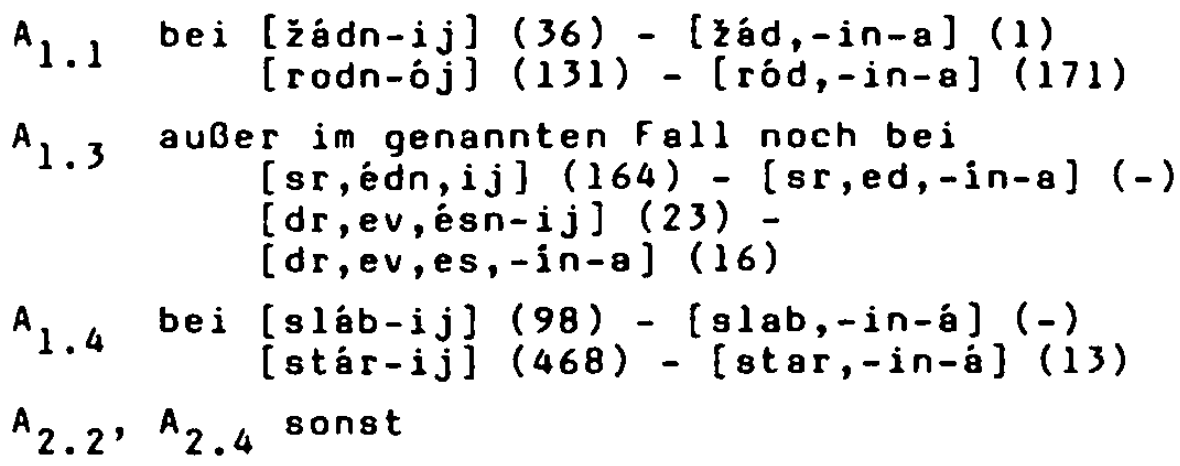

$2 \cdot 4.2 \cdot 2.1 .14 . D-I, D-I I / 1, D-I I I / 7$

$\mathrm{S} / \mathrm{ec} /$

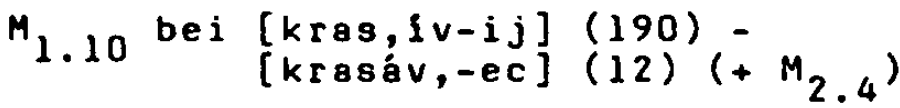

$M_{1.11}$ bei $\left[\begin{array}{l}\text { m,érzk,-ij] } \\ {[m, e r z a ́ v,-e c]}\end{array}(25)\left(+M_{2.4}\right)\right.$

M. 12

$M_{2.4}$ auber in den genannten Fällen noch bei $[s k v, e ́ r n-i j](13)$ -

$[$ skv, ernáv, -ec] (-)

$M_{4}$

$A_{1.2}$ wenn $M_{2.4}$; ouberdem bei

[obórvann-ij] (6) - [oborván,-ec] (-)

$A_{1.3}$ bei zweisilbigem Derivat

$A_{2.2}, A_{2.4}$ sonst

$2 \cdot 4 \cdot 2 \cdot 2 \cdot 1.15 .0-1, D-I I / 1, D-I V / 1$

$S / k_{2} /$

${ }^{M} 1.12$

$M_{2.2}$ bei $\begin{aligned} & {[\text { smúgl-ij] }(14)-[\text { smugl,án-k-a] }} \\ & {[b, e ́ l-i j](28)-[b, e l, a ́ n-k-a](1)}\end{aligned}$

$M_{2.10}$ bei [nov-ij] (1722) - [nov,in-k-a] (19)

(in allen drei hier aufgeführten Paren zusätzlich $M_{4}$ )

$M_{2.19}$ bei $\left[\begin{array}{l}{[v, e r \times n,-i j](112)} \\ {[v, e r \times u ́ s-k-a](-) \text { aber }}\end{array}\right.$

[c,órn-ij] (473)-

[c,ornús $-k-a](-)$ sowie

[durn-oj] (48) -

[durnús-k-a] (1) ohne M 1.12 


$$
\begin{aligned}
& \text { [prost-ój] (287) - [prostús-k-a] (-) }
\end{aligned}
$$

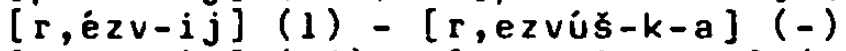

$$
\begin{aligned}
& {[\text { vos, m-ój] (40)-[vos, mús-k-a] }(-)} \\
& {[g n, i l-o j](17)-[g n, i l u ́ s-k-a] \text { (4) }} \\
& \begin{array}{r}
M_{3.1} \text { bei }[n, e ́ z n-i j](50)- \\
{\left[n, \text { ézen-k-a](1) ( } A_{2.1}\right)}
\end{array} \\
& M_{6.1} \\
& A_{1.2} \text { wenn } M_{2} \text {; ferner bei Derivaten, die als } \\
& \text { von Adjektiven mit dem Suffix/č,at/a)- } \\
& \text { geleitet gedacht werden können } \\
& A_{2.2}, A_{2.3} \text { sonst }
\end{aligned}
$$

$2 \cdot 4 \cdot 2 \cdot 2 \cdot 1.16 . D-1, D-11 / 1, D-111 / 7,(D-) 2$

S/ik $/$

$M_{4}$

$$
\begin{aligned}
& A_{1.2} \text { bei }[i z b r a n n-i j](18)-[i z b r a n n,-i k](2) \\
& A_{1.3} \begin{array}{l}
\text { wenn der Stamm des Derivatems auf [ov }] \\
\text { endet }
\end{array} \\
& A_{2.2}, A_{2.4} \text { sonst }
\end{aligned}
$$

$2 \cdot 4 \cdot 2 \cdot 2.1 .17,0-1,0-111 / 7$

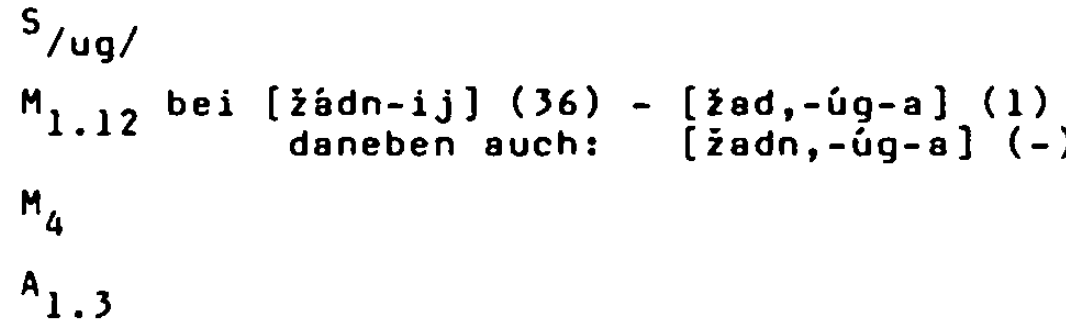

$2.4 .2 .2 .1 .18 .0-1, D-111 / 3,(D-) 2$

$S / \varnothing /$

M.12

$M_{4}, M_{5}$

$A_{2.1}, A_{2.2}, A_{2.5}$

$2.4 .2 .2 .1 .19 .0-1, D-11 / 1, D-111 / 7, D-1 V / 1$

$\mathrm{M}_{5}$

$A_{2.1}$ 
2.4.2.2.2. Die Kombinatorik dieser Ausdrucksmittel

$$
\begin{aligned}
2.4 .2 .2 .2 .1 . & \left(S / u k / ; M_{4} ; A_{1.3}\right) u(D-1) \\
& {[z 1-0 j](87)-[z 1,-u k-a](1) }
\end{aligned}
$$

Anzahl der tatsächlich realisierten Kombinationen: 1

Anzahl der theoretisch maximal möqlichen Kombinationen: $\underline{2}$

- Zusätzlich zu der realisierten ist die Kombination

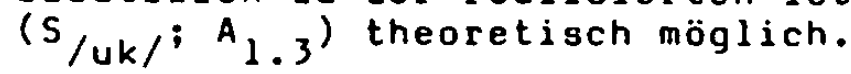

Anzahl der theoretisch minimal möglichen Kombinationen: $\underline{1}$

$2 \cdot 4 \cdot 2 \cdot 2 \cdot 2 \cdot 2 . \quad\left(\mathrm{S} / \mathrm{ok} / \mathrm{M}_{4} ; \mathrm{A}_{1.3}\right) u(\mathrm{D}-\mathrm{I})$

$$
\begin{aligned}
& {[\mathrm{má} 1-i j](224)-[\mathrm{mal},-o k](1)} \\
& \left(\mathrm{S} / \mathrm{ok} / \mathrm{M}_{6.1} ; A_{1.3}\right) u(D-1) \\
& {[d, i k,-i j](6 i)-[d, i c,-o k](-)}
\end{aligned}
$$

Anzahl der tatsächlich realisierten Kombinationen: $\underline{2}$

Anzahl der theoretisch maximal möqlichen Kombinationen: 3

- Zusätzlich zu der realisierten Kombination ist die Kombination $\left(S / o k / A_{1.3}\right)$ theoretisch möglich.

Anzahl der theoretisch minimal möglichen Kombinationen: $\underline{2}$

- Weniger Kombinationen als die tatsächlich realisierten sind zwar theoretisch denkbar, nicht jedoch auch möglich.

$$
\begin{aligned}
& 2.4 .2 .2 .2 .3 . \quad\left(S / i s k / ; A_{1.3}\right) u(D-I) \\
& {[g o ́-i j](73)-[g o l-i \xi k-a](-) } \\
&\left(S / i s ̌ k / M_{1.11} ; A_{1.3}\right) u(D-1) \\
& {[k o r o t k,-i j](11)-[\text { orot-iśk-a] (1) }}
\end{aligned}
$$

Anzahl der tatsächlich realisierten Kombinationen: $\underline{2}$

Anzahl der theoretisch maximal möglichen Kombinationen: $\underline{2}$

Anzahl der theoretisch minimal möglichen Kombinationen: 1

- Theoretisch möglich ist ein Wegfall der Kombination

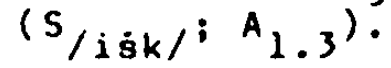

$$
\begin{aligned}
& 2.4 .2 .2 .2 .4 . \quad\left(S / k_{1} / ; A_{2.2}\right) u(D-I) \\
& \text { [bulán-ij] (-) - [bulán-k-o] (-) } \\
& \left(S_{\left./ k_{1} / ; A_{2.5}\right) \cup(D-1)}\right. \\
& {[s, e ́ r-i j](116)-[s, e r-k-o](-)}
\end{aligned}
$$


Anzahl der tatsächlich realisierten Kombinationen: $\underline{2}$

Anzahl der theoretisch maximal möglichen Kombinationen: 2

Anzahl der theoretisch minimal möglichen Kombinationen: $\underline{2}$

$2.4 .2 .2 .2 .5 . \quad\left(S / a c, / ; M_{1.3} ; A_{1.3}\right) u(D-1)$

[bogát-ij] (238)-[bog-áč,] (8)

$\left(S / a c, f ; M_{5} ; A_{1.3}\right) u(D-1)$

[lóvk,-ij] (15)-[lovk-ác, ] (4)

Anzahl der tatsächlich realisierten Kombinationen: 2

Anzahl der theoretisch maximal möglichen Kombinationen: 4

- Zusätzlich zu den realisierten sind die Kombinationen $\left(\mathrm{S} / \mathrm{ac}, / ; M_{15} ; A_{1.3}\right)$ sowie $\left(S_{/ a c ̌, f} A_{1.3}\right)$ theoretisch möglich.

Anzahl der theoretisch minimal möglichen Kombinationen: $\underline{2}$

$2.4 .2 \cdot 2 \cdot 2.6 . \quad\left(S_{/ i 8 / ;} ; A_{1.3}\right) \cup(D-I)$

[durn-ój] (48)-[durn-ís] (-)

$\left(S_{/ i \S /} ; M_{1.11} ; A_{1.3}\right) u(D-1)$

$[k r, e ́ p k,-i j](30)-[k r, e p-i \dot{s}]$

$\left(\mathrm{S} / \mathrm{i}\right.$ / $\left.; \mathrm{M}_{1.11} ; \mathrm{A}_{2.2}\right) \cup(\mathrm{D}-\mathrm{I})$

$[g l a ́ d k,-i j](26)-[g l a ́ d-i z ̌](-)$

$\left(S / i \check{s} / M_{1.12} ; A_{2.2}\right) \cup(D-1)$

[posl,édn,-ij] (664)-[posl,éd-iš (-)

$\left(\mathrm{S} / \mathrm{iš} / ; \mathrm{M}_{6.8} ; \mathrm{A}_{2.2}\right) \cup(\mathrm{D}-\mathrm{I})$

$[m, a ́ g k,-i j](91)-[m, a ́ k,-i s](-)$

Anzahl der tatsächlich realisierten Kombinationen: $\underline{S}$

Anzahl der theoretisch maximal möglichen Kombinationen: $\underline{8}$

- Zusätzlich zu den realisierten sind die Kombinationen

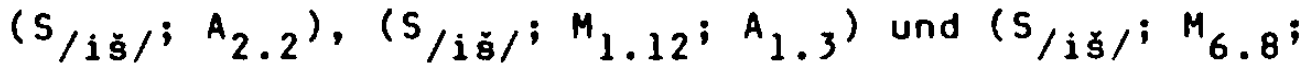
$A_{1.3}$ ) theoretisch möglich.

Anzahl der theoretisch minimal möglichen Kombinationen: 3

- Theoretisch möglich ist ein Wegfall der Kombinationen $\left(S_{/ i s /} ; A_{1.3}\right)$ und $\left(S_{/ i s /} ; M_{1.11} ; A_{2.2}\right)$.

$2 \cdot 4 \cdot 2 \cdot 2 \cdot 2 \cdot 7 . \quad\left(S_{/ a k /} ; A_{1.3}\right) u(D-1)$

[prost-ój] (287)-[prost-ák] 


$$
\begin{aligned}
& \text { (S/ak/; } \left.M_{1.12} ; A_{1.3}\right) u(D-1) \\
& {[c, u d n-o ́ j](5)-[c ̌ c, u d-a ́ k](30)} \\
& \left(\mathrm{S} / \mathrm{ak} / \mathrm{M}_{2.6 \cup 4} ; A_{1.3}\right) u(D-I) \\
& \text { [sm,él-ij] (73) }-[\mathrm{sm}, \mathrm{el}, \grave{c},-\dot{a} k](6) \\
& \left(\mathrm{S} / \mathrm{ak} / \mathrm{M}_{4} ; \mathrm{A}_{1.3}\right) u(D-1) \\
& {[b, e ́ d n-i j](112)-[b, e d n,-\dot{a} k](7)} \\
& \left(\mathrm{S} / \mathrm{ak} / ; \mathrm{M}_{6.1} ; A_{1.3}\right) u(D-I) \\
& \text { [lóvk,-ij] (15)-[lovč,-ák] (-) }
\end{aligned}
$$

Anzahl der tatsächlich realisierten Kombinationen: $\underline{5}$

Anzahl der theoretisch maximal möqlichen Kombinationen: $?$

- Zusätzlich zu den realisierten sind die Kombinationen $\left(\mathrm{S} / \mathrm{ak} / \mathrm{M}_{1.1244} ; \mathrm{A}_{1.3}\right)$ und $\left(\mathrm{S} / \mathrm{ak} / \mathrm{M}_{2.6} ; \mathrm{A}_{1.3}\right)$ theoretisch möglich.

Anzahl der theoretisch minimal möglichen Kombinationen: 3

- Theoretisch möglich ist ein Wegfall der Kombinationen $\left(S_{/ a k} / ; A_{1.3}\right)$ und $\left(S / a k / ; M_{4} ; A_{1.3}\right)$.

$22 \cdot 4 \cdot 2 \cdot 2 \cdot 2 \cdot 8 . \quad\left(S / u x / ; A_{1.3}\right) u(D-I)$

$$
\begin{aligned}
& {[\text { tólst-ij] (69)-[tolst-úx-8] (1) }} \\
& \left(S / u x / ; A_{1.3}\right) \cup(D-I I / 1) \\
& {[g r, \dot{z} n-i j](59)-[g r, 8 z n-i x-a](-)}
\end{aligned}
$$

Anzahl der tatsächlich realigierten Kombinationen: 1

Anzahl der theoretisch maximal möglichen Kombinationen: 1

Anzahl der theoretisch minimal möglichen Kombinationen: 1

$22 \cdot 4 \cdot 2 \cdot 2 \cdot 2.9 .\left(5 / u \dot{s} / ; A_{1.3}\right) \cup(D-11 / 1)$

$$
\begin{aligned}
& \text { [rodn-ój] (131) - [rodn-ís-a] (-) } \\
& \left(\mathrm{S} / \mathrm{us} / ; A_{1.3}\right) \cup(D-1) \\
& {[m, i l-i j](242)-[m, i l-i \dot{s}-a](1)} \\
& \left(S / u s / ; M_{5} ; A_{1.3}\right) \cup(D-I) \\
& \text { [plósk,-ij] (32) - [plosk-ús-a] (-) }
\end{aligned}
$$

Anzahl der tatsächlich realisierten Kombinationen: $\underline{2}$

Anzahl der theoretisch maximal möqlichen Kombinationen: $\underline{2}$

Anzahl der theoretisch minimal möglichen Kombinationen: 1

- Theoretisch möglich wäre ein Wegfall der Kombination $\left(s_{/ u \dot{s} /} ; A_{1.3}\right)$. 
$2 \cdot 4 \cdot 2 \cdot 2 \cdot 2 \cdot 10 .\left(5 / 8 g / M_{4} ; A_{1.3}\right) \cup(D-I)$

$$
\begin{aligned}
& {[b, \text { édn-ij] }(112)-[b, e d n,-a g-a](11)} \\
& \left(S / a g / ; M_{1.7} ; A_{1.3}\right) \cup(D-I I / 1) \\
& {[s, \text { impat,it,n-ij](7)-[s,impat,-ág-a]}}
\end{aligned}
$$

Anzahl der tatsächlich realisierten Kombinationen: 2

Anzahl der theoretisch maximal möglichen Kombinationen: 3

- Zusätzlich zu den realisierten Kombinationen ist die Kombination $\left(S_{/ a g / ;} A_{l .3}\right)$ theoretisch möglich.

Anzahl der theoretisch minimal möglichen Kombinationen: $\underline{2}$

$$
\begin{aligned}
& 2.4 .2 .2 .2 .11 \cdot\left(\mathrm{S} / \mathrm{ul}, / \mathrm{A}_{1.3}\right) \cup(\mathrm{D}-\mathrm{I}) \\
& {[g r, a z n-i j](59)-[g r, a z n-u ́ l,-a](-)} \\
& \left(\mathrm{S} / \mathrm{ul}, 1 ; \mathrm{M}_{1.12} ; A_{1.3}\right) \cup(D-1 \mathrm{I} / 1) \\
& {[\text { kapr,izn-ij] (12) - [kapr,iz-ill,-a] (-) }} \\
& \left(\mathrm{S} / \mathrm{ul}, f ; \mathrm{M}_{1.12 \cup 4} ; \mathrm{A}_{1.3}\right) \cup(\mathrm{D}-\mathrm{I}) \\
& \text { [žádn-ij] (36)-[žad,-01,-8](-) } \\
& \left(\mathrm{S} / \mathrm{ul}, / ; \mathrm{M}_{4} ; \mathrm{A}_{1.3}\right) u(D-1) \\
& {[\check{c}, i s t-i j](225)-[\check{c}, i 8 t,-i ́ l-8](-)}
\end{aligned}
$$

Anzahl der tatsächlich realisierten Kombinationen: 4

Anzahl der theoretisch maximal möglichen Kombinationen: 4

- Zusätzlich zu den realisierten sind keine weiteren Kombinationen möglich.

Anzahl der theoretisch minimal möqlichen Kombinationen: 1

- Theoretisch möglich wäre ein Wegfall aller Kombinationen bis auf die Kombination ( $\left./ / u_{1}, f M_{1.1244} ; A_{1.3}\right)$.

$$
\begin{aligned}
& 2.4 .2 .2 .2 .12 .\left(\mathrm{S} / \mathrm{ic} / \mathrm{M}_{4} ; A_{1.3}\right) \cup(D-I) \\
& {[e d, i n-i j](87)-[e d, i n,-\{c-a](36)} \\
& \left(\mathrm{S} / \mathrm{ic} / ; \mathrm{M}_{4} ; A_{2.1}\right) \cup(D-I 1 / 1) \\
& {[u ́ m n-i j](151)-[u ́ m n,-i c-8](20)} \\
& \left(\mathrm{S} / \mathrm{ic} / \mathrm{M}_{4} ; A_{2.2}\right) \cup(D-11 / 1) \\
& {[\check{C}, u d, e ́ s n-i j](66)-[C, u d, e s n,-i c-8](-)} \\
& \left(\mathrm{S} / \mathrm{ic} / ; \mathrm{M}_{4} ; \mathrm{A}_{2.4}\right) \cup(D-I) \\
& {[t u p-o j](18)-[t u p,-1 c-8](3)} \\
& \left(\mathrm{S} / \mathrm{ic} / \mathrm{M}_{6.1} ; \mathrm{A}_{2.4}\right) \cup(\mathrm{D}-\mathrm{I}) \\
& {[g l u x-\dot{j} j](54)-[g l u s-i c-a](-)}
\end{aligned}
$$

Anzahl der tatsächlich realisierten Kombinationen: 
Anzahl der theoretisch maximal möqlichen Kombinationen: 12

- Zusätzlich zu den realisierten sind die Kombinationen $\left(S / i c / ; A_{1.3}\right),\left(S / i c / ; A_{2.1}\right),\left(S / i c / ; A_{2.2}\right),(S / i c / ;$ $\left.A_{2.4}\right),\left(S / i c / ; M_{6.1} ; A_{1.3}\right),\left(S_{/ i c /} ; M_{6.1} ; A_{2.1}\right),\left(S_{/ i c /} ;\right.$ $M_{6.1} ; A_{2.2}$ ) theoretisch möglich.

Anzahl der theoretisch minimal möglichen Kombinationen: $\underline{4}$

- Ein Wegfall der Kombination ( $/ / i c / M_{4} ; A_{2.4}$ ) ist theoretisch möglich.

$$
\begin{aligned}
& 2.4 .2 .2 .2 .13 .\left(\mathrm{S} / \mathrm{in}_{2} / \mathrm{M}_{1.11 \cup 4} ; \mathrm{A}_{1.3}\right) u(D-I) \\
& {[r, e ́ d k,-i j](80)-[r, e d,-i n-a](-)} \\
& \left(\mathrm{S} / \mathrm{in}_{2} / \mathrm{M}_{1.12 \cup 4} ; A_{1.3}\right) \cup(D-I I / 1) \\
& {[d r, e v, e ́ s n-i j](23)-[d r, e v, e s,-i n-a]} \\
& \left(\mathrm{S} / \mathrm{in}_{2} / \mathrm{M}_{1.12 \cup 4} ; A_{2.2}\right) \cup(D-1) \\
& \text { [gromádn-ij] (152) - [gromád,-in-a] (-) } \\
& \left(\mathrm{S} / \text { in }_{2} / \mathrm{M}_{1.12 \mathrm{U}_{4}} ; A_{1.1}\right) \cup(D-I) \\
& \text { [żádn-ij] (36) - [žád,-in-8] } \\
& \left(\mathrm{S} / \text { in }_{2} ; \mathrm{M}_{2.5} ; A_{2.4}\right) \cup(D-I) \\
& {[\check{c}, u z ̌-o ́ j](171)-[\check{c}, u z ̌ b,-i n-8](10)} \\
& \left(\mathrm{S} / \mathrm{in}_{2} / \mathrm{M}_{\left.2.16 \cup 5 ; \mathrm{A}_{1.2}\right) \cup(D-I)}\right. \\
& {[d, i k,-i j](61)-[d, i k o ́ v,-i n-a](7)}
\end{aligned}
$$

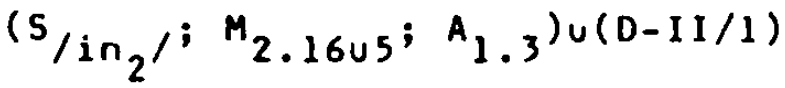

$$
\begin{aligned}
& [k 1, e ́ j k,-i j](5)-[k], e j k o v,-i n-a] \\
& \left(\mathrm{S} / \mathrm{in}_{2} / \mathrm{M}_{4} ; \mathrm{A}_{1.4}\right) \cup(\mathrm{D}-\mathrm{I}) \\
& \text { [stár-ij] (468)-[star,-in-á] (13) } \\
& \left(\mathrm{S} / \text { in }_{2} f ; M_{6.1} ; A_{2.4}\right) \cup(D-I) \\
& \text { [sux-ój] (87) - [8uš-in-8] (-) }
\end{aligned}
$$

Anzahl der tatsächlich realisierten Kombinationen: 9

Anzahl der theoretisch maximal möglichen Kombinationen: 48

- Zusätzlich zu den realisierten sind die Kombinationen
$\left(\mathrm{s} / \mathrm{in}_{2} /\right.$ $\left.A_{1.1}\right)$
$\left(\mathrm{s} / \mathrm{in}_{2}\right)^{;}$
$\left.A_{1.2}\right)$
( $\mathrm{S} /$ in $_{2}{ }^{\prime}$;
$\left.A_{1.3}\right),\left(\mathrm{S} /\right.$ in $_{2} / ;$ 


$$
\begin{aligned}
& \left.A_{1.4}\right),\left(S / \text { in }_{2} / A_{2.2}\right),\left(S / \text { in }_{2} / ; A_{2.4}\right) ;\left(S / i n_{2} / ; M_{1.11 \cup 4} ;\right. \\
& \left.A_{1.1}\right),\left(S / \text { in }_{2} ; M_{1.11 \cup 4} ; A_{1.2}\right),\left(S / \text { in }_{2} ; M_{1.11 \cup 4} ; A_{1.4}\right) \text {, }
\end{aligned}
$$

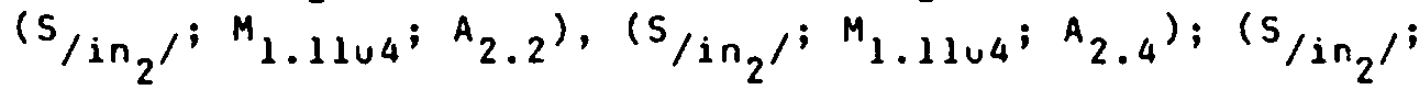

$$
\begin{aligned}
& \left.M_{1.12} ; A_{1.1}\right),\left(S / \text { in }_{2} ; M_{1.12} ; A_{1.2}\right),\left(S / \text { in }_{2} ; M_{1.12} ;\right. \\
& \left.A_{1.3}\right),\left(S / \text { in }_{2} / M_{1.12} ; A_{1.4}\right),\left(S / i n_{2} / M_{1.12} ; A_{2.2}\right) \text {, } \\
& \left(\mathrm{S} / \mathrm{in}_{2} / \mathrm{M}_{1.12} ; \mathrm{A}_{2.4}\right) ;\left(\mathrm{S} / \mathrm{in}_{2} / \mathrm{M}_{1.1204} ; \mathrm{A}_{1.2}\right),\left(\mathrm{S} / \mathrm{in} \mathrm{H}^{\prime} ;\right. \\
& \left.M_{1.1204} ; A_{1.4}\right),\left(S / i n_{2} / M_{1.12 u 4} ; A_{2.4}\right) ;\left(S / i n_{2} / M_{2.5} ;\right. \\
& \left.A_{1.1}\right),\left(S / \text { in }_{2} / M_{2.5} ; A_{1.2}\right),\left(S / \text { in }_{2} / M_{2.5} ; A_{1.3}\right) \text {, } \\
& \left(\mathrm{S} / \mathrm{in}_{2} / \mathrm{M}_{2.5} ; \mathrm{A}_{1.4}\right),\left(\mathrm{S} / \mathrm{in}_{2} / \mathrm{M}_{2.5} ; \mathrm{A}_{2.2}\right) ;\left(\mathrm{S} / \mathrm{in}_{2} / ;\right. \\
& \left.M_{2.1605} ; A_{1.1}\right),\left(S / n_{2} / M_{2.1605} ; A_{1.4}\right),\left(S / \text { in }_{2} / M_{2.1605} ;\right.
\end{aligned}
$$

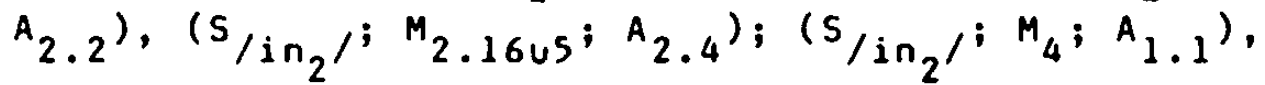

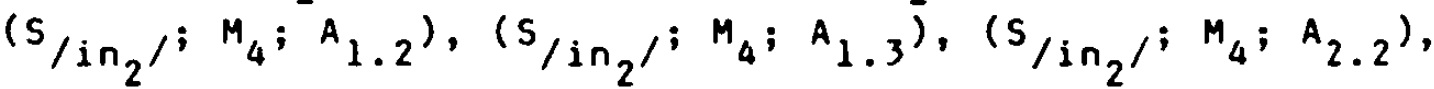

$$
\begin{aligned}
& \left(\mathrm{S} / \mathrm{in} \mathrm{f}^{\prime} ; \mathrm{M}_{4} ; \mathrm{A}_{2.4}\right) ;\left(\mathrm{S} / \mathrm{in}_{2} / \mathrm{M}_{6.1} ; \mathrm{A}_{1.1}\right),\left(\mathrm{S} / \mathrm{in} / \mathrm{i} \mathrm{M}_{6.1} ;\right. \\
& \left.A_{1.2}\right),\left(S / \text { in }_{2} ; M_{6.1} ; A_{1.3}\right),\left(S_{/ i n_{2} /} ; M_{6.1} ; A_{1.4}\right) \text { und } \\
& \left(\mathrm{s} / \mathrm{in}_{2} / \mathrm{M}_{6.1} ; A_{2.2}\right) \text { theoretisch möglich. }
\end{aligned}
$$

Anzahl der theoretisch minimal möglichen Kombinationen: I

- Theoretisch möglich ist ein Wegfall der Kombinationen

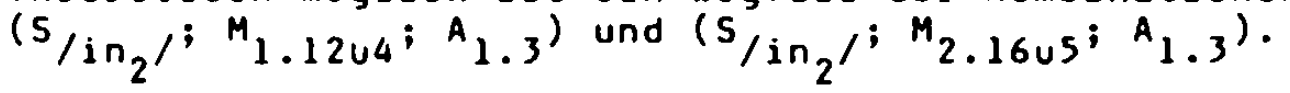

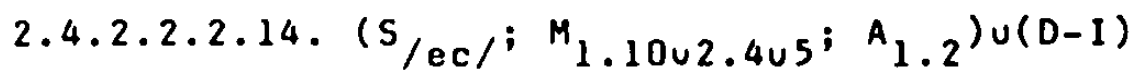

$$
\begin{aligned}
& {[k r a s, i v-i j](190)-[k r a s a ́ v,-e c](12)} \\
& \left(\mathrm{S} / \mathrm{ec} / ; M_{1.11 \cup 2.4} ; A_{1.2}\right) \cup(D-I) \\
& {[m, e r z k,-i j](5)-[m, e r z a ́ v,-e c] \text { (2s) }} \\
& \left(\mathrm{S} / \mathrm{ec} / \mathrm{M}_{\left.1.12 \cup 4 ; A_{2.4}\right) \cup(D-1)}\right. \\
& \text { [xolódn-ij] }(241)-[\text { xolod,-éc] }(-) \\
& \left(\mathrm{S} / \mathrm{ec} / ; \mathrm{M}_{2.4} ; A_{1.2}\right) \cup(D-1 \mathrm{I} / 1) \\
& \text { [skv,érn-ij] (13)-[skv,ernáv, -ec] (-) } \\
& \left(\mathrm{s} / \mathrm{ec} / \mathrm{M}_{4} ; A_{1.3}\right) \cup(D-I) \\
& {[s 1, e p-o j](31)-[81, e p,-e ́ c](4)} \\
& \left(\mathrm{s} / \mathrm{ec} / \mathrm{M}_{4} ; A_{2.2}\right) \cup(D-11 / 1) \\
& \text { [sč, ast], iv-ij] (135) - } \\
& \text { [sč, ast } 1, i v,-e c](10)
\end{aligned}
$$




$$
\begin{aligned}
& \left(\mathrm{S} / \mathrm{ec} / \mathrm{M}_{4} ; \mathrm{A}_{2.2}\right) \cup(D-I 11 / 7) \\
& {[1, u b, i m-i j](79)-[1, u b, i m,-e c](18)}
\end{aligned}
$$

Anzahl der tatsächlich realisierten Kombinationen: 6

Anzahl der theoretisch maximal möqlichen Kombinationen: 19

- Zusätzlich zu den realisierten sind die Kombinationen $\left(\mathrm{S} / \mathrm{ec} / ; \mathrm{M}_{1.12} ; \mathrm{A}_{1.2}\right),\left(\mathrm{S} / \mathrm{ec} / \mathrm{M}_{1.12} ; \mathrm{A}_{1.3}\right),\left(\mathrm{S} / \mathrm{ec} / \mathrm{M}_{1.12}\right.$; $\left.A_{2.2}\right),\left(S / e c / M_{1.12} ; A_{2.4}\right) ;\left(S / e c / M_{1.12 \cup 4} ; A_{1.2}\right)$, $\left(\mathrm{S} / \mathrm{ec} / \mathrm{M}_{1.12 \cup 4} ; A_{1.3}\right),\left(\mathrm{S} / \mathrm{ec} / \mathrm{M}_{1.12 \cup 4} ; \mathrm{A}_{2.2}\right) ;(\mathrm{S} / \mathrm{ec} / ;$ $\left.M_{2.4 \cup 5} ; A_{1.2}\right),\left(S / e c / ; M_{4} ; A_{1.2}\right) ;\left(S / e c / ; A_{1.2}\right),(S / e c / ;$ $\left.\left.A_{1.3}\right), S_{/ e c /} ; A_{2.2}\right)$ und $\left(S / e c / ; A_{2.4}\right)$ theoretisch möglich.

Anzahl der theoretisch minimal möqlichen Kombinationen: $\underline{5}$

- Theoretisch möglich wäre ein Wegfall der Kombination $\left(S_{\text {/ec } /} ; M_{2.4} ; A_{1.2}\right)$.

$$
\begin{aligned}
& 2.4 .2 .2 .2 .15 .\left(S / k_{2} / M_{1.12} ; A_{2.2}\right) u(D-I)
\end{aligned}
$$

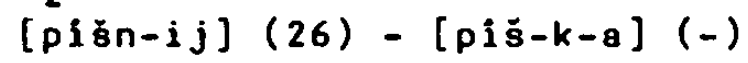

$$
\begin{aligned}
& \left(\mathrm{S} / \mathrm{k}_{2} / \mathrm{M}_{2.2 \cup 4} ; A_{1.2}\right) \cup(D-I) \\
& {[b, e ́ l-i j](28)-[b, e l, a ́ n-k-a](1)} \\
& \left(\mathrm{S} / \mathrm{k}_{2} / \mathrm{M}_{\left.2.10 \cup 4 ; A_{1.2}\right) \cup(D-1)}\right. \\
& \text { [nov-ij] (1722) - [nov,in-k-8] (19) } \\
& \left(\mathrm{S} / k_{2} / ; M_{2.19} ; A_{1.2}\right) u(D-I) \\
& \text { [durn-ój] (48) - [durnús-k-a] (1) }
\end{aligned}
$$

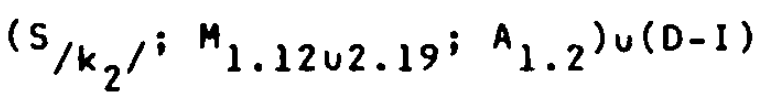

$$
\begin{aligned}
& \text { [દ̌,órn-ij] (473)-[c், ornúš-k-a] (-) } \\
& \left(\mathrm{S} / \mathrm{k}_{2} / \mathrm{M}_{3.1} ; \mathrm{A}_{2.1}\right) \cup(\mathrm{D}-\mathrm{I}) \\
& {[n, e ́ z ̌ n-i j](50)-[n, \dot{z} z \text { en-k-a](1) }} \\
& \left(\mathrm{S} / \mathrm{k}_{2} / \mathrm{M}_{6.1} ; \mathrm{A}_{2.3}\right) \cup(D-I V / 1) \\
& \text { [od,inók, -ij] (4S) - [od, inód, -k-a] (-) } \\
& \left(S / k_{2} ; A_{1.2}\right) \cup(D-1 I / 1) \\
& \text { [vzrivę,at-ij] (1)-[vzrivč,át-k-a] (s) } \\
& \left(S / k_{2} ; A_{2.2}\right) \cup(D-I 1 / 1) \\
& {[8, i v-i j](1)-[3, i v-k-8]}
\end{aligned}
$$


Anzahl der tatsächlich realisierten Kombinationen: 9 Anzahl der theoretisch maximal möglichen Kombinationen: 19

- Zusätzlich zu den realisierten sind die Kombinationen

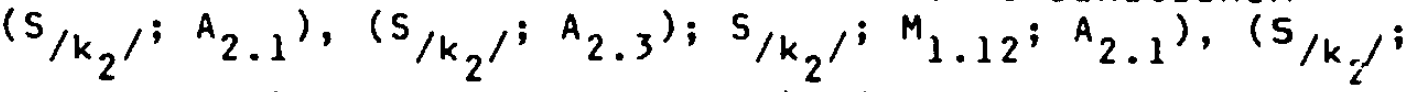

$$
\begin{aligned}
& \left.M_{1.12} ; A_{2.3}\right) ;\left(S / k_{2} / M_{2.2} ; A_{1.2}\right) ;\left(S / k_{2} / M_{2.10} ; A_{1.2}\right) ; \\
& \left(\mathrm{S} / \mathrm{k}_{2} / \mathrm{M}_{3.1} ; \mathrm{A}_{2.2}\right),\left(\mathrm{S} / \mathrm{k}_{2} / \mathrm{M}_{3.1} ; \mathrm{A}_{2.3}\right) ;\left(\mathrm{S} / \mathrm{k}_{2} / \mathrm{M}_{6.1} ;\right. \\
& \left.A_{2.1}\right),\left(S / k_{2} / M_{6.1} ; A_{2.2}\right) \text { theoretisch möglich. }
\end{aligned}
$$

Anzahl der theoretisch minimal möglichen Kombinationen: 6

- Theoretisch möglich ist ein Wegfall der Kombinationen

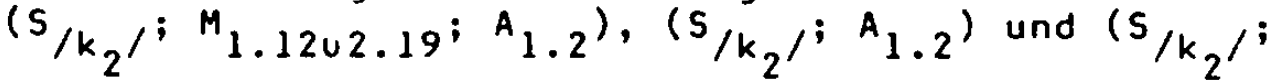

$$
A_{2,2} \text { ). }
$$

$2.4 .2 .2 .2 .16 .\left(S_{/ i k /} ; M_{4} ; A_{1.2}\right) u(0-111 / 7)$

$$
\begin{aligned}
& \text { [izbrann-ij] (18)-[izbránn,-ik] (2) } \\
& \left(\mathrm{S} / \mathrm{ik} / ; \mathrm{M}_{4} ; \mathrm{A}_{1.3}\right) u\left(\left(D_{-}\right) 2\right) \\
& \text { [ć,ornov-ój] (2) - [č,ornov,-ik] (1) } \\
& \left(\mathrm{S} / \mathrm{ik} / ; \mathrm{M}_{4} ; A_{2.2}\right) \cup(D-I I / 1) \\
& \text { [tub,erkul, ozn-ij] (-) - } \\
& \text { [tub,erkul,ózn,-ik] } \\
& \left(S / i k / M_{4} ; A_{2.4}\right) \cup(D-I) \\
& \text { [stár-ij] (468)-[8tar,-ik] (252) }
\end{aligned}
$$

Anzahl der tatsächlich realisierten Kombinationen: 4 Anzahl der theoretisch maximal möglichen Kombinationen: $\underline{8}$

- Zusätzlich zu den realisierten sind die Kombinationen $\left.\left(S_{/ i k /} ; A_{1.2}\right),\left(S_{/ i k /} ; A_{1.3}\right), S_{/ i k /} ; A_{2.2}\right)$ und $(S / i k / ;$ $A_{2.4}$ ) theoretisch möglich.

Anzahl der theoretisch minimal möglichen Kombinationen: 4

$$
\begin{aligned}
& \left.2.4 .2 .2 .2 .17 \text { ( } \mathrm{S}_{/ \mathrm{ug} /} ; \mathrm{M}_{1.12 u 4} ; A_{1.3}\right) u(D-I) \\
& \text { [żádn-ij] (36) - [žad,-úg-a] (1) } \\
& \left(\mathrm{S} / \mathrm{ug} / \mathrm{M}_{4} ; \mathrm{A}_{1.3}\right) \cup(D-111 / 7) \\
& {[p, a ́ n-i j](88)-[p, a n,-i g-a](-)}
\end{aligned}
$$

Anzahl der tatsächlich realisierten Kombinationen: $\underline{2}$ Anzahl der theoretisch maximal möqlichen Kombinationen: $\underline{4}$ 
- Zusätzlich zu den realisierten sind die Kombinationen $\left(S_{/ u g /} ; A_{1.3}\right)$ und $\left(S_{/ u g /} ; M_{1.12} ; A_{1.3}\right)$ theoretisch möglich.

Anzahl der theoretisch minimal möqlichen Kombinationen: 1

- Ein Wegfall der Kombination $\left(S / u g / ; M_{4} ; A_{1.3}\right)$ ist theoretisch möglich.

$2.4 .2 .2 .2 .18 .\left(S / g / M_{1.12} ; A_{2.1}\right) \cup(D-I)$

$$
\begin{aligned}
& \text { [skár,edn-ij] (2)-[skár,ed-ø] (-) } \\
& \left(S / \theta / ; M_{1.12 \cup 5} ; A_{2.1}\right) \cup(D-1) \\
& \text { [or,ig,inál, n-ij] (21) - [or,ig, inál-ø] (10) } \\
& \left(\mathrm{S} / 0 / ; \mathrm{M}_{1.12 \cup 4} ; A_{2.2}\right) \cup((D-) 2) \\
& {[n, \text { eudóbn-ij] }(3)-[n, \text { eudób, }-\emptyset-e](-)} \\
& \left(\mathrm{S} / \theta / ; \mathrm{M}_{4} ; \mathrm{A}_{2.5}\right) \cup(D-1 \mathrm{II} / 3) \\
& \text { [žil-ój] }(24)-[\check{z} i 1,-\emptyset-o ́]
\end{aligned}
$$

Anzahl der tatsächlich realisierten Kombinationen: 4

Anzahl der theoretisch maximal möglichen Kombinationen: 11

- Neben den realisierten sind die Kombinationen ( $S / \sigma / ;$ $\left.A_{2.1}\right),\left(S / \emptyset / A_{2.2}\right),\left(S / \varnothing / A_{2.5}\right) ;\left(S / \emptyset / M_{1.12} ; A_{2.2}\right)$, $\left(S / \varnothing / ; M_{1.12} ; A_{2.5}\right) ;\left(S / D / ; M_{1.12 \cup 4} ; A_{2.5}\right)$ und $(S / \theta / ;$ $M_{4} ; A_{2.2}$ ) theoretisch möglich.

Anzahl der theoretisch minimal möqlichen Kambinationen: $\underline{3}$

- Theoretiech möglich ist ein Wegfall der Kombination $\left(S_{/ 0} ; M_{1.12} ; A_{2.1}\right)$.

$2 \cdot 4 \cdot 2 \cdot 2 \cdot 2 \cdot 19 \cdot\left(A_{2.1}\right) \cup(D-I)$

$$
\begin{aligned}
& {[s m, e ́ l-i j](73)-[8 m, e ́ l i j](-)} \\
& \left(A_{2,1}\right) \cup(D-I I / 1) \\
& \text { [part,ijn-ij] (158) - [part,ijnij] (-) } \\
& \left(A_{2.1}\right) \cup(D-111 / 7) \\
& \text { [vzrósl-ij] (80)-[vzróslij](-) } \\
& \left(A_{2,1}\right) \cup(D-I V / 1) \\
& {[s, e d, m-o j j](34)-[8, e d, m a ́ j a](-)} \\
& \left(A_{2.1} ; M_{s}\right) \cup(D-1) \\
& \text { [sládk, -ij] (33)-[8 ládkoe] (-) }
\end{aligned}
$$

Anzahl der tatgächlich realisierten Kombinationen: 2

Anzahl der theoretisch maximal möglichen Kombinationen: $\underline{2}$ 
Anzahl der theoretisch minimal möqlichen Kombinationen: 1

- Theoretisch möglich ist ein Wegfall der Kombination $\left(A_{2,1}\right) \cup(\ldots)$.

Zur Bildung von Substantiven mit der allgemeinen Explikation - Jemand (etwas), der (das) sich durch ein bestimmtes Merknal auszeichnet ' verwirklicht das Russische somit

$$
\begin{array}{r}
1+2+2+2+2+5+5+1+2+2+4+5+9+6+9 \\
+4+2+4+2=69
\end{array}
$$

unterschiedliche Kombinationen der zur Verfügung stehenden Ausdrucksmittel.

Theoretisch maximal möglich sind

$$
\begin{aligned}
2+3+2+2+4+8+7+1+2+3+4+12+48+19 \\
+19+8+4+11+2=151
\end{aligned}
$$

unterschiedliche Kombinationen dieser Mittel.

Theoretisch minimal möglich sind

$$
\begin{aligned}
& 1+2+1+2+2+3+3+1+1+2+1+4+7+5+3 \\
& +4+1+3+1=50
\end{aligned}
$$

unterschiedliche Kombinationen.

Im Rahmen der deadjektivischen Derivation von Substantiven ${ }^{\prime \prime}$ nutzt das Russische, wie gezeigt, insgesant

$$
21+19+69=109
$$

unterschiedliche Kombinationen der im analytischen Teil dieser Arbeit je für sich beschriebenen Mittel aus.

Theoretisch maximal möglich sind

$$
45+40+151=236
$$

unterschiedliche Kombinationen dieser Ausdrucksmittel.

Theoretisch minimal möglich sind

$$
16+15+50=81
$$

unterschiedliche Kombinationen.

19 Vgl. zu allen Zahlenangaben die Seiten 105, 109 (und 124). 
2.4.3. Deadjektivische Adjektive

2.4.3.1. Gradation des mit dem ableitenden Adjektiv benannten Merkmals

2.4.3.1.1. Hervortreten des Merkmals in mäBiger Form

2.4.3.1.1.1. Aufzählung der Derivatemtypen und der jeweils miteinander kompatiblen Mittel zur Bildung von Adjektiven mit dieser allgemeinen Expliketion

$2 \cdot 4 \cdot 3 \cdot 1.1 .1 .1 .0-I I / 1$

$P / n, e b, e z /$

$A_{2,1}$

$2.4 .3 .1 .1 .1 .2 . \quad D-I, D-I I / 1$

P/po/

$A_{2.1}$

$2.4 .3 .1 .1 .1 .3, D-I, D-I I / 1, D-I I I / 7$

S/ovat/

M. 14

A.3.2

2.4.3.1.1.2. Die Kombinatorik dieser Mittel

$2.4 .3 .1 .1 .2 .1 . \quad\left(P / n, e b, e z / ; A_{2.1}\right) \cup(D-11 / 1)$

[osnovát,el,n-ij] (8).

$[n, e b, e z-o s n o v a ́ t, e l, n-i j]$

Anzahl der tatsëchlich realigierten Kombinationen: 1

Anzahl der theoretisch maximal möqlichen Kombinationen: $\underline{1}$

Anzahl der theoretisch minimal möqlichen Kombinationen: 1

$2.4 .3 .1 .1 .2 .2 . \quad\left(P / p o / ; A_{2.1}\right) \cup(D-I)$

$$
[s, i l, n,-e ́ e](237)^{20}-[p o-s, i l, n,-e ́ e]
$$

20 Um auch solche Bildungen erfassen zu können, die sonst als suppletiv gelten müBten, wird hier nicht der Lexikoneintrag, sondern die form des synthetischen Komparativs als Bezugggröße gewählt. 


$$
\begin{aligned}
& \left(P / p o / ; A_{2.1}\right) \cup(D-I I / 1) \\
& \quad[\text { int,er,ésn,-ee] }(145)- \\
& {[\text { po-int,er,ésn,-ee] (-) }}
\end{aligned}
$$

Anzahl der tatsächlich realisierten Kombinationen: 1

Anzahl der theoretisch maximal möglichen Kombinationen: 1

Anzahl der theoretisch minimal möglichen Kombinationen: 1

$2.4 \cdot 3.1 \cdot 1 \cdot 2.3 . \quad\left(\mathrm{S} /\right.$ ovat $\left./ ; A_{1.3 .2}\right) u(D-1)$

[grúb-ij] (61) - [grub-ovát-ij] (3)

(S/ovat/; $\left.A_{1} .3 .2\right) u(D-111 / 7)$

$[p, a ́ n-i j](88)-[p, a n-o v a ́ t-i j](-)$

(S/ovat/; $\left.M_{1.14} ; A_{1.3 .2}\right) \cup(D-I I / 1$

[rózov-ij] (49)-[roz-ovát-ij] (3)

Anzahl der tatsächlich realisierten Kombinationen: $\underline{2}$

Anzahl der theoretisch maximal möglichen Kombinationen: $\underline{2}$

Anzahl der theoretisch minimal möqlichen Kombinationen: $\underline{l}$

- Theoretisch möglich ist ein Wegfall der Kombination (S/ovat/; $A_{1.3 .2}$ ).

Zur Bildung von Adjektiven mit der voratehenden allgemeinen Explikation ('Hervortreten des Merkmals in mäBiger Form') verwirklicht das Russische

$$
1+1+2=4
$$

unterschiedliche Kombinationen der zur Verfügung stehenden Mittel.

Theoretisch maximal möglich sind ebenfalls

$$
1+1+2=4
$$

unterschiedliche Kombinationen dieser Mittel.

Theoretisch minimal möglich sind

$$
1+1+1=3
$$

unterschiedliche Kombinationen.

2.4.3.1.2. Hervortreten des Merkmals in verstärkter form

$$
\begin{aligned}
& \text { 2.4.3.1.2.1. Aufzählung der Derivatemtypen und der jeweils } \\
& \text { miteinander kompatiblen Mittel zur Bildung von } \\
& \text { Adjektiven mit dieser allgemeinen Explikation }
\end{aligned}
$$


$2.4 .3 .1 .2 .1 .1 . D-11 / 1, D-111 / 7$

$P / p, e r, e /$

$A_{2.1}$

$2 \cdot 4 \cdot 3 \cdot 1 \cdot 2 \cdot 1.2 . D-1, D-I I / 1$

S/uð̌, /

$M_{4}(/ 1 / \sim / 1, /$ regelmäBig; sonst als freie Variante)

$A_{1.3}$

2.4.3.1.2.2. Die Kombinatorik dieser Mittel

$$
\begin{aligned}
& 2.4 .3 .1 .2 .2 .1 . \quad(P\left./ p, e r, e / ; A_{2.1}\right) u(D-I I / 1) \\
& {[i z v, e ́ s t n-i j](339)-} \\
& {[p, e r, e-i z v, e ́ s t n-i j](-) } \\
&\left(P / p, e r, e / ; A_{2.1}\right) u(D-I I I / 7) \\
&/ v o z b u z d, o n n-i j](17)- \\
& {[p, e r, e-v o z b u z ̌ d, o ́ n n-i j](-) }
\end{aligned}
$$

Anzahl der tatsächlich realisierten Kombinationen: 1

Anzahl der theoretisch maximal möglichen Kombinationen: 1

Anzahl der theoretisch minimal möqlichen Kombinationen: $\underline{1}$

$2 \cdot 4 \cdot 3 \cdot 1 \cdot 2 \cdot 2 \cdot 2 . \quad\left(\mathrm{S} / \mathrm{us̆c̆,/;} A_{1,3}\right) \cup(D-1)$

$[$ bol, ś-ój] (2066)-[bol, ś-úšc, -ij]

$\left(S / u \check{c}\right.$ ć, $\left.; M_{4} ; A_{1.3}\right) \cup(D-I)$

$[x, i t r-i j](38)-[x, i t r,-i u s c ̌ c,-i j](1)$

$\left(S / u \check{B} \check{c}, / ; M_{4} ; A_{1.3}\right) \cup(D-1 I / 1)$

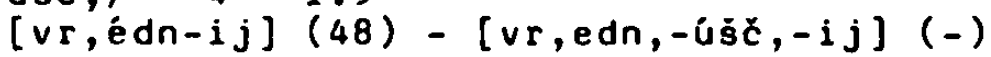

Anzahl der tatsächlich realisierten Kombinationen: $\underline{2}$

Anzahl der theoretisch maximal möglichen Kombinationen: $\underline{2}$

Anzahl der theoretisch minimal möglichen Kombinationen: 1

- Theoretisch möglich ist ein Wegfall der Kombination $\left(\mathrm{S} / \mathrm{us̆c}, f ; A_{1.3}\right)$.

Zur Bildung von Adjektiven mit der allgemeinen Explikation 'Hervortreten des Merkmals in verstärkter Form' verwirklicht das Russische

$$
1+2=3
$$


unterschiedliche Kombinationen der zur Verfügung stehenden Mittel.

Theoretisch maximal möglich sind gleichfalls

$$
1+2=3
$$

unterschiedliche Kombinationen.

Theoretisch minimal möglich sind

$$
1+1=2
$$

unterschiedliche Kombinationen dieser Mittel.

2.4.3.1.3. Hervortreten des Merkmals in höchster oder in übermäBiger Form

2.4.3.1.3.1. Aufzählung der Derivatemtypen und der jeweils miteinander kompatiblen Mittel zur Bildung von Adjektiven mit dieser allgemeinen Explikation

$2 \cdot 4 \cdot 3.1 .3 .1 .1 . D-1, D-11 / 1$

P/arx,i/

$$
A_{2.1}
$$

$2.4 .3 .1 .3 .1 .2 .(D-) 1.3$

P/nai/

$A_{2.1}$

$2 \cdot 4 \cdot 3 \cdot 1 \cdot 3 \cdot 1 \cdot 3 \cdot \quad D-1$

P/pr,e/

$A_{2.1}$

$2.4 .3 .1 .3 .1 .4 ., D-1, D-11 / 1$

$P / s v$,erx/

$A_{2.1}$

$2.4 .3 .1 .3 .1 .5 .0-1,0-11 / 1$

P/sup,er/

$A_{2.1}$

$2.4 .3 .1 .3 .1 .6 . D-1, D-11 / 1, D-111 / 6,(D-) 2$

P/raz/

${ }^{2} 2.1$ 
$2.4 .3 .1 .3 .1 .7 . D-I, D-I I / 1$

P/ul,tral

$A_{2.1}$

$2.4 \cdot 3 \cdot 1 \cdot 3 \cdot 1 \cdot 8 . \quad D-I$

S/enn/

$M_{4}, M_{6.1}$

$A_{1.3}$

$2.4 .3 .1 .3 .1 .9 . D-I, D-I I / 1, D-I I I / 7, D-V / 3$

$\mathrm{S} / \check{s} /$

$M_{1.11}\left(+M_{2.1 \cup 6.6} ; A_{1.2}\right)$ bei $[b 1, i z k,-i j](215)$ -

$[n, i z k,-i j](1, i 6)-[n, i z ̌ a j-s ̌ b-i j](-)$

$M_{2.1}$ auBer in den genannten fällen nur denn, wenn $M_{6.1}$ oder $M_{6.11}$

$M_{2.8}$

$\mathrm{M}_{4}$

$M_{6.1}$

$M_{6.11}\left(+M_{2.1} ; A_{1.2}\right)$ bei

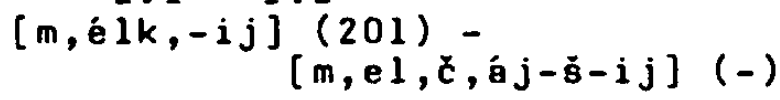

$A_{1.2}$ wenn der Stamm des Derivatems einsilbig ist; auberdem bei

[bogát-ij] (175)

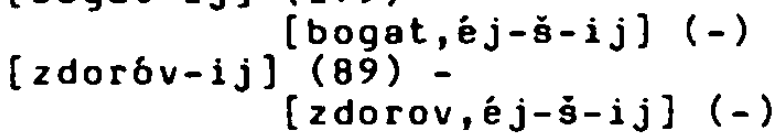

$A_{2.2}, A_{2.3}$ sonst

$2 \cdot 4 \cdot 3 \cdot 1 \cdot 3 \cdot 1 \cdot 10.0-1, D-I I / 1$

PuS/za ... onn/

$\mathrm{M}_{4}$

$A_{1.3}$ 
2.4.3.1.3.2. Die Kombinatorik dieser Mittel

$2.4 .3 .1 .3 .2 .1 . \quad\left(P / a r x, i / ; A_{2.1}\right) u(D-I)$

$[g l u p-i j](99)-[a r x, i-g l u p-i j](-)$

$\left(P /\right.$ arx,ili $\left.A_{2.1}\right) \cup(D-I I)$

$[g, e n, i a j, n-i j](11)-$

$[a r x, i-g, e n, i a ́ l, n-i j](-)$

Anzahl der tatsächlich realisierten Kombinationen: 1

Anzahl der theoretisch maximal möglichen Kombinationen: $\underline{1}$

Anzahl der theoretisch minimal möglichen Kombinationen: $\underline{1}$

$2.4 .3 .1 .3 .2 .2 . \quad\left(P / n a i / A_{2.1}\right) u((D-) 1.3)$

[važn, éjjz-ij] (-) -

$[n a i-v a z ̌ n, e ́ j s ̌ s-i j](-)^{21}$

Anzahl der tatsächlich realisierten Kombinationen: 1

Anzahl der theoretisch maximal möglichen Kombinationen: 1

Anzahl der theoretisch minimal möglichen Kombinationen: $\underline{1}$

$2.4 \cdot 3.1 .3 .2 .3 .\left(\mathrm{P} / \mathrm{pr}, \mathrm{e} / ; \mathrm{A}_{2.1}\right) U(\mathrm{D}-\mathrm{I})$

$[m, i 1-i j](242)-[p r, e-m, i 1-i j]$

Anzahl der tatsächlich realisierten Kombinationen: 1

Anzahl der theoretisch maximal möglichen Kombinationen: 1

Anzahl der theoretisch minimal möglichen Kombinationen: 1

$2.4 \cdot 3.1 \cdot 3 \cdot 2 \cdot 4 . \quad\left(\mathrm{P} / \mathrm{sv}, \mathrm{erx} / \mathrm{A}_{2.1}\right) \cup(\mathrm{D}-\mathrm{I})$

$$
[d a ́ l, n,-i j](125)-[s v, e r x-d a ́ 1, n,-i j]
$$

$\left(P / s v, e r x / ; A_{2.1}\right) \cup(D-11 / 1)$

[sovr,em,énn-ij] (261) -

[sv, erx-sovr,em,énn-ij]

Anzahl der tatsächlich realisierten Kombinationen: 1

Anzahl der theoretisch maximal möglichen Kombinationen: 1

Anzahl der theoretisch minimal möqlichen Kombinationen: $\underline{1}$

21 Um auch solche Bildungen erfassen zu können, die sonst als suppletiv gelten müBten, wird hier statt der Grundform die Form des einfachen Komparativs gewählt. 
$2.4 .3 .1 .3 .2 .5 . \quad\left(P / s u p, e r / ; A_{2.1}\right) u(D-I)$

$$
\text { [slóžn-ij] (239) - [sup,er-slóžn-ij] (-) }
$$

$\left(P /\right.$ sup,er/; $\left.A_{2.1}\right) \cup(D-I I / 1)$

$[\operatorname{modn}-i j](12)-[\sup , e r-m o ́ d n-i j](-)$

Anzahl der tatsächlich realisierten Kombinationen: 1

Anzahl der theoretisch maximal möglichen Kombinationen: 1

Anzahl der theoretisch minimal möglichen Kombinationen: 1

$2 \cdot 4 \cdot 3 \cdot 1 \cdot 3 \cdot 2 \cdot 6 . \quad\left(P / \mathrm{raz}_{1} ; \mathrm{A}_{2.1}\right) \cup(D-I)$

$[v, e s, o ́ l-i j](183)-[\mathrm{raz}-v, e 8,61-i j](-)$

$\left(P /\right.$ razl; $\left.A_{2.1}\right) \cup(D-I I / 1)$

[kudr,áv-ij] (9) - [raz-kudr,áv-ij] (-)

$\left(P / \mathrm{raz} ; \mathrm{A}_{2.1}\right) \cup(D-1 \mathrm{II} / 6)$

$[1, u b, e ́ z n-i j](18)-$

$[\mathrm{r} a z-1, u b, e ́ z n-i j](-)$

$\left(P /\right.$ raz/; $\left.A_{2.1}\right) \cup\left(\left(D_{-}\right) 2\right)$

$[n, e s c \dot{c} \operatorname{stn}-i j](50)-$

[raz-n,esč,ástn-ij] (-)

Anzahl der tatä̈chlich realisierten Kombinationen: 1

Anzahl der theoretisch maximal möglichen Kombinationen: 1

Anzahl der theoretisch minimal möglichen Kombinationen: $\underline{1}$

$2 \cdot 4 \cdot 3 \cdot 1 \cdot 3 \cdot 2 \cdot 7 . \quad\left(P / u l, t r a / ; A_{2.1}\right) u(D-1)$

$[1, e ́ v-i j](i 17)-[u l, t r a-1, \dot{e} v-i j](-)$

$\left(P / u l, t r a / ; A_{2.1}\right) u(D-11 / 1)$

[r,evol,uciónn-ij] (225) -

[ul, tra-r,evol, uciónn-ij](-)

Anzahl der tatsächlich realisierten Kombinationen: 1

Anzahl der theoretisch maximal möglichen Kombinationen: 1

Anzahl der theoretisch minimal möglichen Kombinationen: 1

$2.4 \cdot 3 \cdot 1 \cdot 3 \cdot 2 \cdot 8 . \quad\left(\mathrm{S} / \mathrm{enn} / M_{1.12} ; A_{1.3}\right) \cup(D-I)$

[strášn-ij] (173) - [straś-énn-ij] (1)

$\left(\mathrm{S} / \mathrm{enn} / \mathrm{M}_{4} ; A_{1.3}\right) \cup(D-1)$

[tólst-ij] (69)-[tolst, -énn-ij] (-)

$\left(\mathrm{s} / \mathrm{enn} / ; M_{6.1} ; A_{1.3}\right) u(D-1)$

[ ̧̇irók, -ij] (312)-[širod, -énn-ij] (3) 
Anzahl der tatsächlich realisierten Kombinationen: 3

Anzahl der theoretisch maximal möglichen Kombinationen: 5

- Zusätzlich zu den realisierten sind die Kombinationen $\left(S_{/ e n n / ;} A_{1.3}\right)$ und $\left(S_{/ e n n / ;} M_{1.124} ; A_{1.3}\right)$ theoretisch möglich.

Anzahl der theoretisch minimal möglichen Kombinationen: $\underline{3}$

$2 \cdot 4 \cdot 3 \cdot 1 \cdot 3 \cdot 2 \cdot 9$

$$
\begin{aligned}
& \left(S / 5 / ; M_{\left.1.11 \cup 2.1 \cup 6.6 ; A_{1.2}\right) \cup(D-I)}\right. \\
& {[n, i z k,-i j](116)-[n, i z a ́ j-\$-i j](-)} \\
& \left(\mathrm{s} / \mathrm{s} / ; M_{\left.2.1 \cup 6.1 ; A_{1.2}\right) u(D-1)}\right.
\end{aligned}
$$

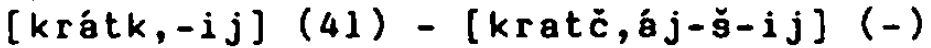

$$
\begin{aligned}
& \left(\mathrm{S} / \check{\mathrm{s}} / ; \mathrm{M}_{2.1 \cup 6.11} ; \mathrm{A}_{1.2}\right) \cup(\mathrm{D}-\mathrm{I}) \\
& {[m, e ́ 1 k,-i j](201)-[m, e l, \grave{c}, \dot{a} j-\check{s}-i j](-)} \\
& \left(\mathrm{S} / \S / ; M_{2.8 \cup 4} ; A_{1.2}\right) \cup(D-I) \\
& {[g r u b-i j](6 l)-[g r u b, e j-z-i j](-)} \\
& \left(\mathrm{S} / \check{\mathrm{g}} / \mathrm{M}_{2.8 \cup 4} ; \mathrm{A}_{2.2}\right) \cup(\mathrm{D}-\mathrm{II} / 1) \\
& \text { [užásn-ij] (64)-[užásn,ej-šs-ij] (-) } \\
& \left(\mathrm{S} / \mathrm{s} / ; \mathrm{M}_{2.8 \cup 4} ; A_{2.2}\right) \cup(D-11 \mathrm{I} / 7) \\
& \text { [ub,ežd, bnn-ij] (21) - } \\
& {[u b, e z ̌ d, o ́ n n, e j-\check{z}-i j](-)} \\
& \left(\mathrm{S} / \dot{\mathrm{s}} / ; \mathrm{M}_{2.8 \cup 4} ; A_{2.2}\right) \cup(D-V / 3) \\
& \text { [vs, evozmóżn-ij] (17) - } \\
& \text { [vs, evozmóżn, ej-š-ij] (-) } \\
& \left(\mathrm{S} / \dot{\mathrm{s} /} ; \mathrm{M}_{2.8 \cup 4} ; A_{2.3}\right) u(D-I) \\
& \text { [ozorn-bj] (11) - [ozorn,éj-ś-ij] (-) }
\end{aligned}
$$

Anzahl der tatsächlich realisierten Kombinationen: 6

Anzahl der theoretisch maximal möglichen Kombinationen: 13

- Zusätzlich zu den realisierten sind die Kombinationen $\left(S_{/ 8 /} ; M_{2.8} ; A_{1.2}\right),\left(S_{/ s /} ; M_{2.8} ; A_{2.2}\right),\left(S / \check{s} / ; M_{2.8} ;\right.$ $\left.A_{2.3}\right) ;\left(S / s / ; M_{2.106 .1} ; A_{2.2}\right),\left(S / 8 / ; M_{2.106 .1} ; A_{2.3}\right) ;$

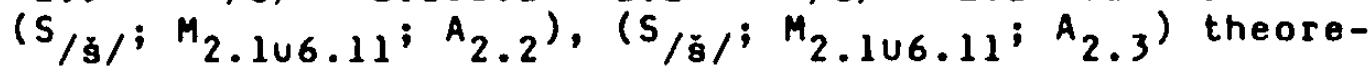
tisch möglich.

Anzahl der theoretisch minimal möglichen Kombinationen: 5

- Ein Wegfall der Kombination ( $/ / \dot{s} / ; M_{2.844 ;} A_{1.2}$ ) ist theoretisch möglich.

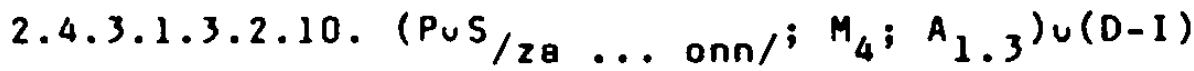

$$
[k, 1 s i-i j](13)-[2 a-k, i s 1,-o ́ n n-i j](-)
$$




$$
\begin{gathered}
\left(P u S / z a, \ldots \text { onn } / M_{4} ; A_{1.3}\right) \cup(D-I I / 1) \\
{[g r, a z n-i j](59)-} \\
{[z a-g r, a z n,-o n n-i j](-)}
\end{gathered}
$$

Anzahl der tatsächlich realisierten Kombinationen: $\underline{1}$

Anzahl der theoretisch maximal möqlichen Kombinationen: 1

Anzahl der theoretisch minimal möglichen Kombinationen: $\underline{1}$

Somit verwirklicht das Russische zur Bildung von Adjektiven mit der allgemeinen Explikation 'Hervortreten des Merkmals in höchster oder in übermäBiger form'

$$
1+1+1+1+1+1+1+3+6+1=17
$$

unterschiedliche Kombinationen der zur Verfügung stehenden Ausdrucksmittel.

Theoretisch maximal möglich sind

$$
1+1+1+1+1+1+1+5+13+1=26
$$

unterschiedliche Kombinationen.

Theoretisch minimal möglich sind

$$
1+1+1+1+1+1+1+3+5+1=16
$$

unterschiedliche Kombinationen dieser Mittel.

2.4.3.2. Negierung oder Nichtvorhandensein des mit dem ableitenden Adjektiv bezeichneten Merkmals oder Ausdruck der Entgegengesetztheit zu diesem

2.4.3.2.1. Aufzählung der Derivatemtypen und der jeweils miteinander kompatiblen Mittel zur Bildung von Adjektiven mit dieser allgemeinen Explikation

$2.4 \cdot 3 \cdot 2.1 .1 .0-11 / 1$

$$
\begin{aligned}
& \mathrm{P} / \mathrm{a} / \\
& \mathrm{A}_{2.1}
\end{aligned}
$$

$2.4 \cdot 3.2 \cdot 1.2 . D-1, D-11 / 1$

Plant,i/

$A_{2.1}$

$2 \cdot 4 \cdot 3 \cdot 2 \cdot 1.3 . D-1, D-11 / 1$

$P / b, e z /$

$A_{2.1}$ 
2.4.3.2.1.4. D-I, D-II/I

P/im/bzw. P/ir/vor /r/, /r, /

$A_{2.1}$

$2.4 .3 .2 .1 .5,0-1, D-11 / 1,0-111 / 6$

$P / n, e /$

$A_{2.1}$

$2.4 .3 .2 .1 .6 . D-1, D-11 / 1$

P/prot,ivo/

$A_{2.1}$

2.4.3.2.2. Die Kombinatorik dieser Mittel

$2.4 .3 .2 .2 .1 . \quad\left(P / a / ; A_{2.1}\right) \cup(D-1 I / 1)$

[morál,n-ij] (66) - [a-morál,n-ij] (8)

Anzahl der tatsächlich realisierten Kombinationen: 1

Anzahl der theoretisch maximal möglichen Kombinationen: 1

Anzohl der theoretisch minimal möqlichen Kombinationen: $\underline{1}$

$2 \cdot 4 \cdot 3.2 .2 .2 . \quad\left(P /\right.$ ant,$\left.i / A_{2.1}\right) u(D-I)$

[sociál,n-ij] (142) - [ant,i-sociál,n-ij](-)

$\left(P /\right.$ ant,$\left.i / A_{2.1}\right) \cup(D-I I / 1)$

[istor, $\{\dot{c}, e s k,-i j](167)-$

$[$ ant, i-istor, $\{\grave{c}, e s k,-i j](-)$

Anzahl der tatsächlich realisierten Kombinationen: 1

Anzahl der theoretisch maximal möglichen Kombinationen: $\underline{1}$

Anzahl der theoretisch minimal möglichen Kombinationen: 1

2.4.3.2.2.3. $\left(P / b, e z / ; A_{2.1}\right) \cup(D-I)$

[grámotn-ij] (20)-[b,ez-grámotn-ij]

$$
\begin{aligned}
& \left(P / b, e z / ; A_{2.1}\right) u(D-I I / l) \\
& {[\grave{c}, e l o v, e ́ c \dot{c}, n-i j](5)-} \\
& {[b, e z-c ̌ c, \theta l o v, e ́ c ̌ c, n-i j]}
\end{aligned}
$$

Anzahl der tatsächlich realisierten Kombinationen: $\underline{1}$ Anzahl der theoretisch maximal möglichen Kombinationen: 1 
Anzahl der theoretisch minimal möglichen Kombinationen: $\underline{1}$

$2.4 .3 .2 .2 .4 \cdot\left(P_{/ \mathrm{ir} /} ; \mathrm{A}_{2.1}\right) \cup(\mathrm{D}-\mathrm{I})$

$[r, e a ́ l, n-i j](83)-[i r-r, e a ́ l, n-i j](-)$

$\left(P / i m / ; A_{2.1}\right) \cup(D-11 / 1)$

$[\operatorname{morá} l, n-i j](66)-[i m-\operatorname{mor} \mathbf{l}, n-i j](-)$

Anzahl der tatsächlich realisierten Kombinationen: 1

Anzahl der theoretisch maximal möqlichen Kombinationen: 1

Anzahl der theoretisch minimal möglichen Kombinationen: $\underline{1}$

$2.4 .3 .2 .2 .5 . \quad\left(P / n, e / ; A_{2.1}\right) u(D-I)$

$[b o l, \dot{s}-\delta j](2066)-[n, e-b o l, \grave{s}-o ́ j](198)$

$\left(P / n, e / ; A_{2.1}\right) \cup(D-I I / 1)$

$[$ naúč, $n-i j](291)-[n, e-n a u ́ c ̌, n-i j](-)$

$\left(P / n, e / ; A_{2.1}\right) \cup(D-I I 1 / 6)$

$[o b, i t a ́ e m-i j](-)-[n, e-o b, i t a ́ e m-i j](-)$

Anzahl der tatsächlich realisierten Kombinationen: 1

Anzahl der theoretisch maximal möglichen Kombinationen: 1

Anzahl der theoretisch minimal möglichen Kombinationen: 1

$2.4 \cdot 3 \cdot 2 \cdot 2.6 . \quad\left(P / p r o t, i v o / ; A_{2.1}\right) u(D-I)$

[est,éstv,enn-ij] (43) -

[prot, ivo-est,éstv,enn-ij]

(P/prot,ivo/; $\left.A_{2.1}\right) \cup(D-I I / 1)$

$[n a u ́ z, n-i j](291)-[p r o t, i v o-n a u ́ c, n-i j](-)$

Anzahl der tatsächlich realisierten Kombinationen: 1

Anzahl der theoretisch maximal möglichen Kombinationen: 1

Anzahl der theoretisch minimal möglichen Kombinationen: 1

Zur Bildung von Adjektiven mit der allgemeinen Explikation

' Negierung oder Nichtvorhandensein des mit dem ableitenden Adjektiv bezeichneten Merkmols oder Ausdruck der Entgegengesetztheit zu diesem' verwirklicht das Russische

$$
1+1+1+1+1+1=6
$$

unterschiedliche Kombinationen der zur Verfügung stehenden Ausdrucksmittel.

Theoretisch maximal wie auch minimal möglich sind gleichfalls je sechs unterschiedliche Kombinationen dieser Mittel. 
2.4.3.3. Expressive Nuancierung des Merkmals und gleichzeitiges Hervortreten desselben in verstärkter Form

2.4.3.3.1. Aufzählung der Derivatemtypen und der jeweils miteinander kompatiblen Mittel zur Bildung von Adjektiven mit dieser allgemeinen Explikation

$2.4 \cdot 3 \cdot 3.1 .1 .0-1$

S/us, en,k,/

$M_{1.11}$ sofern der Stamm des Derivatems auf /k/ oder / k, / nach Konsonant endet

$M_{4}$

A.3.1

$2.4 \cdot 3 \cdot 3 \cdot 1.2 . \quad D-1, D-11 / 1$

S/oxon,k,/ v /ošen,k,/

$M_{1.11}$ sofern der Stamm des Derivatems auf /k/ oder / k, / nach Konsonant endet

$M_{4}$ nicht betroffen sind die Hinterzungenlaute

A.3.1

$2.4 \cdot 3.3 .1 .3 . D-1, D-11 / 1, D-111 / 7$

$\mathrm{S} / \mathrm{en,k,/}$ nach Hinterzungenlauten auch S/on,k,/

Ml.11 sofern der Stamm des Derivatems auf

/k/ oder / $k, /$ nach Konsonant endet;

Ausnahme: [žalk,-ij] (34) -

[žálk-on, $k,-i j](-)$

$M_{4}, M_{5}$

$M_{6.7}$ bei [mál-ij] (224)-[máx-on,k,-ij]

$A_{2,2}: A_{2.3}$

2.4.3.3.2. Die Kombinatorik dieser Mittel

$2.4 .3 .3 .2 .1 . \quad\left(\mathrm{S} / \mathrm{us}, \mathrm{en,k,f} ; \mathrm{M}_{4} ; \mathrm{A}_{1.3 .1}\right) u(\mathrm{O}-\mathrm{I})$

[mál-ij] (224)-[mal, -ís,en,k,-ij] (4)

(S/us,en,k,/; $\left.M_{1.11 u 4 ;} A_{1.3 .1}\right) \cup(D-1)$

[tónk,-ij] (162)-[ton,-ús,en,k,-ij] (-)

Anzahl der tatsächlich realisierten Kombinationen: $\underline{2}$

Anzahl der theoretisch maximal möglichen Kombinationen: 4 
- Zusätzlich zu den realisierten sind die Kombinationen $\left(\mathrm{S} / \mathrm{s}, \mathrm{en,k,f} ; \mathrm{A}_{1.3 .1}\right),\left(\mathrm{S} / \mathrm{us,en,k,/} \mathrm{H}_{1.11} ; \mathrm{A}_{1.3 .1}\right)$ the oretisch möglich.

Anzahl der theoretisch minimal möglichen Kombinationen: 1

- Ein Wegfall der Kombination ( $S / u s, e n, k, f ; M_{4} ; A_{1.3 .1}$ ) ist theoretisch möglich.

$2 \cdot 4 \cdot 3 \cdot 3.2 .2 . \quad\left(S / o x a n, k, / ; M_{1.11} ; A_{1.3 .1}\right) \cup(D-1)$

$$
[1, o ́ g k,-i j](152)-[1, o g-o ́ x o n, k,-i j](-)
$$

$\left(\mathrm{S} /\right.$ osen,k, $\left.; \mathrm{M}_{4} ; \mathrm{A}_{1.3 .1}\right) \cup(\mathrm{D}-1)$

[skór-ij] (45) - [skor, -óšen,k,-ij] (-)

(S/oxon,k,/; $\left.M_{4} ; A_{1.3 .1}\right) \cup(D-I I / 1)$

[sv,éti-ij] $(116)-$

$[s v, e t],-o x o n, k,-i j](-)$

Anzahl der tatsächlich realisierten Kombinationen: 2

Anzahl der theoretisch maximal möglichen Kombinationen: 4

- Zusätzlich zu den realisierten sind die Kombinationen

(S/oxon,k,/ v /ošen,k,/; $A_{1.3 .1}$ ), (S/oxon,k,/v /ošen,k,f; $M_{1.1144} ; A_{1.3 .1}$ ) theoretisch möglich.

Anzahl der theoretisch minimal möglichen Kombinationen: $\underline{2}$

$2 \cdot 4 \cdot 3 \cdot 3 \cdot 2 \cdot 3 . \quad\left(S / e n, k, / ; M_{4} ; A_{2.2}\right) u(D-I)$

[kor,ič,n,ev-ij] (23) -

[kor, ič, n,ev, -en,k, -ij] ( - )

$\left(\mathrm{S} / \mathrm{en,k,f} ; M_{4} ; A_{2.2}\right) \cup(D-I I / 1)$

[pušist-ij] (26) - [pušist, -en,k,-ij] (-)

$\left(\mathrm{S} / \mathrm{en,k,f} ; \mathrm{M}_{4} ; \mathrm{A}_{2.3}\right) \cup(\mathrm{D}-\mathrm{I})$

$[b i, e ́ d n-i j](4 S)-[b l, e ́ d n,-e n, k,-i j](-)$

$\left(S / e n, k, f ; M_{4} ; A_{2.3}\right) \cup(D-111 / 7)$

$[p, a ́ n-i j](88)-[p, a ́ n,-e n, k,-i j]$

$\left(S / e n, k, / ; M_{1.11 \cup 4} ; A_{2.3}\right) \cup(D-1)$

$[\dot{u} z k,-i j](102)-[i z,-e n, k,-i j](14)$

$\left(\mathrm{s} / \mathrm{on,k,f} ; \mathrm{M}_{5} ; \mathrm{A}_{2.3}\right) u(D-I)$

[visók,-ij] (549)-[visók-on,k,-ij] (-)

$\left(\mathrm{S} / \mathrm{on,k,f} ; \mathrm{M}_{6.7} ; \mathrm{A}_{2.3}\right) \cup(\mathrm{D}-\mathrm{I})$

$[m a ́ l-i j](224)-[m a ́ x-o n, k,-i j]$

Anzahl der tatsächlich realisierten Kombinationen: 5

Anzahl der theoretisch maximal möglichen Kombinationen: $\underline{9}$ 
- Zusätzlich zu den reslisierten sind die Kombinationen $\left(S / e n, k, / v / o n, k, / ; A_{2.2}\right),\left(S / e n, k, / v / o n, k, / ; A_{2.3}\right) ;$ $\left(S / e n, k, / v\right.$ lon,k,f; $\left.M_{1.11} ; A_{2.3}\right) ;\left(S / e n, k, f ; M_{5} ; A_{2.2}\right)$ theoretisch möglich.

Anzahl der theoretisch minimal möglichen Kombinationen: 4

- Ein Wegfall der Kombination (S/en,k,/ v/on,k, ; $M_{4}$; $\left.A_{2.3}\right)$ ist theoretisch möglich.

Zur Bildung von Adjektiven mit der allgemeinen Explikation 'Expressive Nuancierung des Merkmals und gleichzeitiges Hervortreten desselben in verstärkter Form' verwirklicht dos Russische

$$
2+2+5=9
$$

unterschiedliche Kombinationen der zur Verfügung stehenden Ausdrucksmittel.

Theoretisch maximal möglich sind

$$
4+4+9=17
$$

unterschiedliche Kombinationen.

Theoretisch minimal möglich sind

$$
1+2+4=7
$$

unterschiedliche Kombinationen dieser Mittel.

Im Rahmen der deadjektivischen Derivation von Adjektiven 22 nutzt das Russische, wie gezeigt, insgesemt

$$
4+3+17+6+9=37
$$

unterschiedliche Kombinationen der im analytischen Teil dieser Arbeit je für sich beschriebenen Mittel aus.

Theoretisch maximal möglich sind

$$
4+3+26+6+17=56
$$

unterschiedliche Kombinationen.

Theoretisch minimal möglich sind

$$
3+2+16+6+7=34
$$

unterschiedliche Kombinationen dieser Ausdrucksmittel.

22 Vgl. zu den Zahlenangaben die Seiten 126, 127f, 133, 135 (und 138). 
2.4.4. Deadjektivische Verben

2.4.4.1. Das mit dem ableitenden Adjektiv benannte Merkmal annehmen oder an den Tag legen

2.4.4.1.1. Aufzählung der Derivatemtypen und der jeweils miteinander kompatiblen Ausdrucksmittel zur Bildung von Verben mit dieser allgemeinen Explikation

$2 \cdot 4 \cdot 4 \cdot 1 \cdot 1.1 .0-1$

S/a/

$M_{4}$ bei [róvn-ij] (4l)-[rovn,-a-t,] (-)

$M_{6.1}, M_{6.11}$

$A_{1.3}$

2.4.4.1.1.2. D-I, D-II/1, D-II/2, D-II/12, D-II/14

$S / e /$

M1.11

$M_{1.12}$ aber: [vláżn-ij] (24) -

$[$ [ lažn, $-\dot{e}-t],(-)$

[gristn-ij] (36) -

[grustn, $-\dot{e}-t],(-)$

$[1, e d$, an-ój\} $(48)$ -

$[1, e d, e n,-\dot{e}-t$,$] (2)$

$M_{1.16}$

$M_{3.3}$ bei $[1, e d, a n-o ́ j](48)-$

$1, e d, e n,-\dot{e}-t],(2)$

$M_{4}$

$M_{6.1}$ bei $[m, a ́ g k,-i j](91)-[m, a g \grave{c},-\dot{e}-t],(-)$

$A_{1.3}$

$A_{2.1}$ a) wenn $D-11 / 14$

b) wenn der Stamm des Derivatems mehrsilbig ist und auf -aB-, -תяB-, -aT-, -еват-, -ив- oder auf - ИВH- endet

Ausnahmen:

[bagróv-ij] (13) - [bagrov, -s-t, ] (2)

[bogát-ij] (238) - [bogat, -ée-t, ] (2)

[zdorov-ij] (89)-[zdorov, $-\dot{e}-t],(-)$

$[1, i \operatorname{lov}-i j](12)-[1, i 10 v,-\dot{e}-t$,$\} (1)$

$[$ puncóv-ij] (2) - [puncov, -é-t,] (-)

[rózov-ij] (49) - [rozov, -é-t, ] (3) 
c) bei

$$
\begin{aligned}
& \text { [b,er,ém,enn-aja] (4) - } \\
& \text { [postil-ij] (s) [b,er,ém,en,-e-t,] (-) } \\
& \text { [rum,án-ij] (11) - postil, }-e-t,] \text { ( } \\
& {[u g r, u m-i j](21)-[u g r, u m,-e-t,\}(-)}
\end{aligned}
$$

$2.4 .4 .1 .1 .3 . D-I, D-I I / 1, D-V / 2$

$$
\begin{aligned}
& S / i_{1} / \\
& M_{1.11} \text { bei [skól, zk, -ij] (4) }- \\
& {[s k o l, z,-i-t,](18)}
\end{aligned}
$$$$
M_{1.12} ; M_{4} ; M_{5} ; M_{6.1}
$$$$
A_{1.3} \text { bei [skól, 2k,-ij] (4) - }
$$$$
[\text { skol, } z,-i-t,](18)
$$

$A_{2.2}, A_{2.4}$ sonst

$2.4 .4 .1 .1 .4 . D-1, D-11 / 2$

$$
\mathrm{SuPo}_{1.13} ; M_{4}, 8, a / 1
$$$$
A_{1.2} \text { bei [visók,-ij] (549) - }
$$

$A_{2.2} ; A_{2.4}$ sonst

$2.4 .4 .1 .1 .5, D-1, D-11 / 1, D-111 / 2,(D-) 2$

$\mathrm{S} / \mathrm{n}, \mathrm{ic}, \mathrm{a} /$

$M_{1.11}, M_{1.12}$

$A_{2.1}$

$2.4 \cdot 4 \cdot 1 \cdot 1.6 .0-1$

$$
\begin{aligned}
& S / n u / \\
& M_{1.11} \text { aber }[g o ́ r, k,-i j](43)-[\text { górk-nu-t, ] (-) } \\
& M_{1.15} ; M_{3.5} ; M_{5} \\
& A_{1.2}
\end{aligned}
$$

$2.4 .4 .1 .1 .7 . D-1, D-11 / 1$

S/oval'

$M_{1.7}, M_{1.12} ; M_{2.13}$

$M_{4}$ wenn $M_{2.13}$ 


$$
\begin{aligned}
& A_{1.2} \text { wenn } M_{2.13} \\
& A_{1.3 .2} \text { sonst; aber [dosádn-ij] (4) - } \\
& {[\text { dosád-ova-t,] (3) }}
\end{aligned}
$$

$2.4 .4 .1 .1 .8, D-I, D-I I / 1, D-V / 4, D-V / S,(D-) 2$

S/atrova/

$M_{1.12} ; M_{3.1}$

$A_{2.1}$

2.4.4.1.2. Die Kombinatorik dieser Mittel

$$
\begin{aligned}
& 2 \cdot 4 \cdot 4 \cdot 1.2 .1 . \quad\left(\mathrm{S} / \mathrm{a} / ; \mathrm{A}_{1.3}\right) \cup(D-I) \\
& {[x \operatorname{rom}-\delta j](10)-[x \operatorname{rom}-\dot{a}-t,](10)} \\
& \left(S / 8 / M_{4} ; A_{1.3}\right) \cup(D-1) \\
& \text { [róvn-ij] (41)-[rovn,-á-t,] (-) } \\
& \left(\mathrm{S} / \mathrm{a} / \mathrm{M}_{6.1} ; \mathrm{A}_{1.3}\right) \cup(\mathrm{D}-\mathrm{I}) \\
& \text { [dorog-ój] (224) - [doroż-á-t,] (1) } \\
& \left(S / a / ; M_{6.1} ; A_{1.3}\right) \cup(D-I) \\
& {[d, i k,-i j](61)-[d, i c h,-\dot{a}-t,](-)} \\
& \left.(S / a) ; M_{6.1} ; A_{1.3}\right) \cup(D-I) \\
& {[v, e ́ t x,-i j](13)-[v, e t \dot{s}-\dot{a}-t,](-)} \\
& \left(\mathrm{s} / \mathrm{a} / \mathrm{M}_{6.11} ; A_{1.3}\right) \cup(D-1) \\
& {[m, \dot{e} 1 k,-i j](202)-[m, e l, \dot{c},-\dot{a}-t,]}
\end{aligned}
$$

Anzahl der tatsëchlich realisierten Kombinationen: 4

Anzahl der theoretisch maximal möglichen Kombinationen: 4

Anzahl der theoretisch minimal möglichen Kombinationen: $\underline{3}$

- Theoretisch möglich ist ein Wegfall der Kombination $\left(S_{/ a^{\prime}} ; A_{1.3}\right)$.

$2 \cdot 4.4 \cdot 1.2 .2 . \quad\left(5 / e / ; M_{1.11 \cup 4} ; A_{1.3}\right) \cup(D-I)$

$$
\begin{aligned}
& {[r, e ́ d k,-i j](80)-[r, e d,-\dot{e}-t,] \text { (7) }} \\
& \left(\mathrm{S} / \mathrm{e} / ; \mathrm{M}_{1.12} ; \mathrm{A}_{2.1}\right) \cup(D-I I / 14) \\
& {[b, e s s, i 1, n-i j](16)-} \\
& {[b, e s s, 11,-e-t,](-)} \\
& \left(\mathrm{S} / \mathrm{e} / \mathrm{M}_{1.16 \cup 4} ; A_{1.3}\right) \cup(D-I) \\
& \text { [russk,-ij] (514)-[rus,-é-t,] (-) } \\
& \left(\mathrm{s} / \mathrm{e} / \mathrm{M}_{3.3 \cup 4} ; \mathrm{A}_{1.3}\right) \cup(\mathrm{D}-\mathrm{II} / 1) \\
& {[1, e d, a n-o ́ j](48)-[1, e d, e n,-\dot{e}-\dot{t},]}
\end{aligned}
$$




$$
\begin{aligned}
& \left(S / e / ; M_{4} ; A_{1.3}\right) \cup(D-1) \\
& {[b, e ́ l-i j](471)-[b, e 1,-e ́-t,](-)} \\
& \left(s / e / ; M_{4} ; A_{2.1}\right) \cup(D-11 / 1) \\
& \text { [volosát-ij] (11)-[volosát, -e-t,] (-) } \\
& \left(\mathrm{S} / \mathrm{e} / \mathrm{M}_{4} ; A_{2.1}\right) u(D-11 / 12) \\
& \text { [brónzov-ij] (34) - [brónzov, -e-t,] (-) } \\
& \left(\mathrm{S} / \mathrm{e} / \mathrm{M}_{6.1} ; \mathrm{A}_{1.3}\right) \cup(\mathrm{D}-\mathrm{I}) \\
& {[m, a ́ g k,-i j](91)-[m, a g c ̌,-\dot{e}-t,](-)}
\end{aligned}
$$

Anzahl der tatsächlich realisierten Kombinationen: I

Anzahl der theoretisch maximal möglichen Kombinationen: 13

- Zusätzlich zu den realisierten sind die Kombinationen $\left(\mathrm{S} / \mathrm{e} / \mathrm{M}_{1.11} ; \mathrm{A}_{1.3}\right),\left(\mathrm{S} / \mathrm{e} / \mathrm{M}_{1.12} ; \mathrm{A}_{1.3}\right) ;(\mathrm{S} / \mathrm{e} / ;$ $\left.M_{1.12 \cup 4} ; A_{1.3}\right),\left(S_{/ e} ; M_{1.1204} ; A_{2.1}\right) ;\left(\mathrm{S} / e / M_{1.16} ;\right.$ $\left.A_{1.3}\right)$ und $\left(S_{/ e} ; M_{3.344} ; A_{2.1}\right)$ theoretisch möglich.

Anzahl der theoretisch minimal möglichen Kombinationen: 6

- Theoretisch möglich ist ein Wegfall der Kombination $\left.(\mathrm{S} / \mathrm{e}) ; \mathrm{M}_{4} ; \mathrm{A}_{1.3}\right)$.

$$
\begin{aligned}
& 2.4 .4 .1 .2 .3 . \quad\left(S / i_{1} / ; A_{2.2}\right) \cup(D-11 / 1)
\end{aligned}
$$

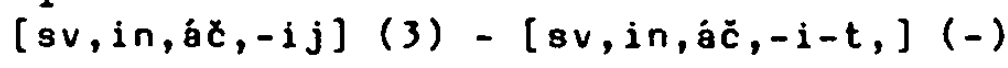

$$
\begin{aligned}
& \left(\mathrm{S} / \mathrm{i}_{1} / \mathrm{M}_{1.11 \cup 4} ; \mathrm{A}_{1.3}\right) \cup(D-1) \\
& \text { [skól,zk,-ij] (4) - [skol,z,-i-t,] (18) } \\
& \left(S / i_{1} / M_{1.12 \cup 4} ; A_{2.4}\right) \cup(D-I) \\
& \text { [grustn-ij] (36) - [grust, }-i-t,] \text { (6) } \\
& \left(S / i_{1} / ; M_{4} ; A_{2.4}\right) \cup(D-I) \\
& {[g l u p-i j](99)-[g l u p,-i-t,](1)} \\
& \left(\mathrm{S} / \mathrm{i}_{1} / \mathrm{M}_{5 \cup 6.1} ; \mathrm{A}_{2.4}\right) \cup(D-I) \\
& {[g o ́ r, k,-i j](43)-[g \circ c \check{c},-j-t,](1)} \\
& \left(S / i_{1} / M_{6.1} ; A_{2.2}\right) \cup(D-V / 4) \\
& {[k o s o b o ́ k,-i j](1)-[k o s o b o ́ c,-i-t,](-)} \\
& \left(S / i_{1} / M_{6.1} ; A_{2.4}\right) u(D-1) \\
& \text { [lóvk,-ij] (15)-[lovc , -i-t,] }
\end{aligned}
$$


Anzahl der tatsächlich realisierten Kombinationen: ? Anzahl der theoretisch maximal möglichen Kombinationen: 23

- Zusätzlich zu den realisierten sind die Kombinationen
$\left(S / i_{1} / ; A_{1.3}\right),\left(S / i_{1} / A_{2.4}\right) ;\left(S / i_{1} / M_{1.1104} ; A_{2.2}\right)$,
$\left(S_{/ i_{1}} / M_{1.11 \cup 4} ; A_{2.4}\right) ;\left(S_{/ i_{1} /} ; M_{1.11} ; A_{1.3}\right),\left(S / i_{1} / ;\right.$
$\left.M_{1.11} ; A_{2.2}\right),\left(S / i_{1} / M_{1.11} ; A_{2.4}\right) ;\left(S / i_{1} / M_{1.12 u 4} ;\right.$
$\left.A_{1.3}\right),\left(S / i_{1} / M_{1.1204} ; A_{2.2}\right) ;\left(S / i_{1} / M_{1.12} ; A_{1.3}\right)$,
$\left(S_{/ i_{1} /} ; M_{1.12} ; A_{2.2}\right),\left(S / i_{1} / M_{1.12} ; A_{2.4}\right) ;\left(S / i_{1} / ; M_{4} ;\right.$
$\left.A_{1.3}\right),\left(S / i_{1} / M_{4} ; A_{2.2}\right) ;\left(S / i_{1} / M_{S \cup 6.1} ; A_{1.3}\right)$ sowie $\left(\mathrm{S} / \mathrm{i}_{1} / \mathrm{M}_{\text {SU6.1 }} ; \mathrm{A}_{2.4}\right.$ ) theoretisch möglich.

Anzahl der theoretisch minimal möglichen Kombinationen: 4

- Theoretisch möglich ist ein Wegfall der Kombinationen

$$
\left(S_{/ i_{1} /} ; A_{2.2}\right) ;\left(S / i_{1} / M_{4} ; A_{2.4}\right) ;\left(S / i_{1} / M_{6.1} ; A_{2.4}\right) \text {. }
$$

$2.4 .4 .1 .2 .4 . \quad\left(S_{4 P o} / i \ldots s, a / 1 ; M_{1.13 \cup 4} ; A_{1.2}\right) \cup(D-1)$

$$
\begin{aligned}
& {[v i s o k,-i j](549)-[v i s,-i-t,-8, a] \text { (3) }} \\
& \text { (SuPo/i ...s, a/ ; } \left.M_{4} ; A_{2.2}\right) \cup(D-1)
\end{aligned}
$$$$
\text { [rázn-ij] (34i) - [rázn,-i-t,-s,a] }
$$

$$
\left(S u P o / i \ldots s, a / 2 ; M_{4} ; A_{2.2}\right) \cup(D-I I / 2)
$$

[kudr,áv-ij] (9) - [kudr,áv, $-i-t,-s, a](-)$

$$
\begin{aligned}
& (\text { SuPo } / i \\
& \left.\quad[x \cup d-a j](3 / a) ; M_{4} ; A_{2,4}\right) \cup(D-I) \\
& (32)-[x u d,-i-t,-s, a](-)
\end{aligned}
$$

Anzahl der tatsächlich realisierten Kombinationen: 3

Anzahl der theoretisch maximal möglichen Kombinationen: $\underline{9}$

- Zusätzlich zu den realisierten sind die Kombinationen

$$
\left(S_{P O} / i \ldots s, a / 1 ; A_{1.2}\right),\left(S_{1} P_{/ i} \ldots s, a / 2 ; A_{2.2}\right) \text {, }
$$

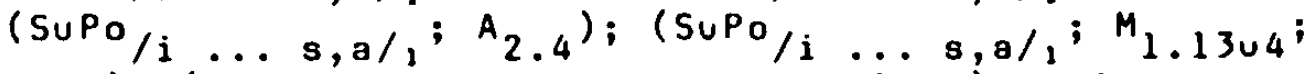

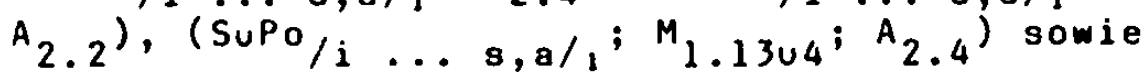

(SuPo/i ...s,a/2; $M_{4} ; A_{1.2}$ ) theoretisch möglich.

Anzahl der theoretisch minimal möglichen Kombinationen: $\underline{3}$

$$
\begin{aligned}
& 2 \cdot 4 \cdot 4 \cdot 1.2 .5 . \quad\left(\mathrm{S} / \mathrm{n}, \mathrm{ič,a} ; \mathrm{M}_{1.11} ; \mathrm{A}_{2.1}\right) \cup(D-I)
\end{aligned}
$$

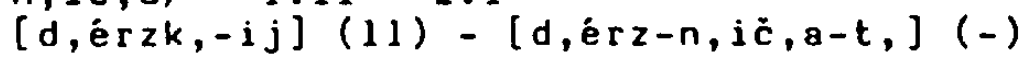

$$
\begin{aligned}
& \left(\mathrm{S} / \mathrm{n,ic}, \mathrm{a} / \mathrm{M}_{1.12} ; \mathrm{A}_{2.1}\right) \cup(\mathrm{D}-\mathrm{I}) \\
& {[\text { sol, idn-ij] (23)-[sol, id-n,ić,a-t, ] (-) }}
\end{aligned}
$$




$$
\begin{aligned}
& \left(S / n, i c, a / ; M_{1.12} ; A_{2.1}\right) \cup(D-I I / 1) \\
& {[g, e n, i a ́ l, n-i j](11)-}
\end{aligned}
$$

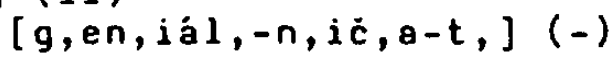

$$
\begin{aligned}
& \left(S / n, i c ̌, a / M_{1.12} ; A_{2.1}\right) \cup(D-I I I / 2) \\
& \text { [skritn-ij] (5) - [skrit-n,ič,a-t, ] (-) } \\
& \left(S / n, i c ̌, 8 / M_{1.12} ; A_{2.1}\right) \cup((D-) 2) \\
& \text { [b,esstidn-ij] (3) - }
\end{aligned}
$$

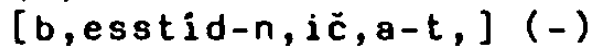

Anzahl der tatsächlich realisierten Kombinationen: $\underline{2}$ Anzahl der theoretisch maximal möglichen Kombinationen: 3

- Zusätzlich zu den realisierten ist die Kombination

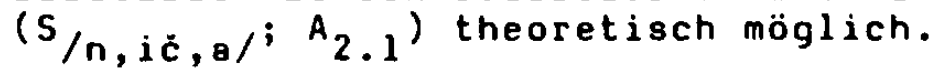

Anzahl der theoretisch minimal möglichen Kombinationen: $\underline{2}$

$2 \cdot 4 \cdot 4 \cdot 1 \cdot 2 \cdot 6$

$$
\begin{aligned}
& \left(\mathrm{S} / \mathrm{nu} / A_{1.2}\right) \cup(D-I) \\
& {[s 1, e p-o j](31)-[81, e ́ p-n u-t,](1)} \\
& \left(\mathrm{S} / \mathrm{nu} / ; M_{1.11} ; A_{1.2}\right) \cup(D-1) \\
& {[k r, e ́ p k,-i j](70)-[k r, e ́ p-n u-t,](17)} \\
& \left(\mathrm{S} / \mathrm{nu} / \mathrm{M}_{1.15} ; A_{1.2}\right) \cup(D-1) \\
& {[\text { mókr-ij] (66) - [mók-nu-t,] (-) }} \\
& \left(\mathrm{S} / \mathrm{nu} / ; \mathrm{M}_{3.5} ; A_{1.2}\right) u(D-I) \\
& \text { [glux-oj] (54)-[glox-nu-t, ] (1) } \\
& \left(\mathrm{S} / \mathrm{nu} / \mathrm{M}_{5 u s} ; A_{1.2}\right) \cup(D-I) \\
& {[g o r, k,-i j](43)-[g o ́ r k-n u-t,](-)}
\end{aligned}
$$

Anzahl der tatsächlich realisierten Kombinationen: $\underline{5}$

Anzahl der theoretisch maximal möglichen Kombinationen: $\underline{7}$

- Zusätzlich zu den realisierten sind die Kombinationen

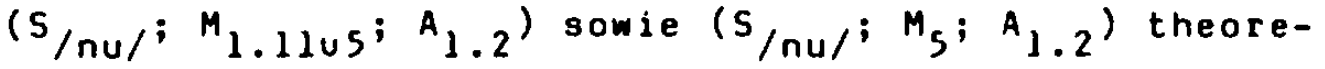
tisch möglich.

Anzahl der theoretisch minimal möglichen Kombinationen: 4

- Theoretisch möglich ist ein Wegfall der Kombination $\left(\mathrm{s} / \mathrm{nu} / \mathrm{A}_{1.2}\right)$.

$$
\begin{aligned}
2.4 .4 .1 .2 .7 . & \left(S_{/ o v a} / A_{1.3 .2}\right) u(D-1) \\
& {[\text { pust-ój] }(123)-[\text { pust-ová-t, ] }}
\end{aligned}
$$




$$
\begin{aligned}
& \left(S_{\text {/ova }} / ; M_{1.742 .13} ; A_{1.2}\right) u(D-I I / 1) \\
& \text { [sx,emat, ic, n-ij] (1) } \\
& {[s x, e m a t, i z, i r-o v a-t,](-)} \\
& \left(S / 0 a_{1} / M_{1.12} ; A_{1.2}\right) \cup(D-I 1 / 1) \\
& \text { [dosádn-ij] (4) - [dosád-ova-t, ] (3) } \\
& \left(S_{\text {/ova }} / ; M_{1.12} ; A_{1.3 .2}\right) \cup(D-11 / 1) \\
& \text { [ozorn-ój] (11)-[ozor-ová-t,] } \\
& \text { (S/ova } / ; M_{\left.1.12 \cup 2.13 \cup 4 ; A_{1.2}\right) \cup(D-1 I / 1)} \\
& \text { [sablónn-ij] (3) - } \\
& \text { [šablon, iz, ir-ova-t, ] (-) }
\end{aligned}
$$

Anzahl der tatsächlich realisierten Kombinationen: $\underline{5}$

Anzahl der theoretisch maximal möqlichen Kombinationen: $\underline{6}$

- Zusätzlich zu den realisierten ist die Kombination ( /ova $_{1} ; M_{1.1202 .13} ; A_{1.2}$ ) theoretisch möglich.

Anzahl der theoretisch minimal möglichen Kombinationen: 3

- Theoretisch möglich ist ein Wegfall der Kombinationen $\left(S_{\text {/ova }} / ; A_{1.3 .2}\right)$ und $\left(S_{\text {/ova }} / ; M_{1.12} ; A_{1.2}\right)$.

$2.4 .4 \cdot 1.2 .8 . \quad\left(5 / 8\right.$ trova/; $\left.A_{2.1}\right) u(D-I)$

$$
\begin{aligned}
& \text { [sv,ir,ép-ij] }(11)- \\
& {[s v, i r, e ́ p-s t v o v a-t,](-) } \\
&\left(s / s t v o v a / ; M_{1.12} ; A_{2.1}\right) \cup(D-I I / 1)
\end{aligned}
$$$$
[u m n-i j](151)-[u m-s t v o v a-t,](1)
$$$$
\text { (S/strova); } \left.M_{1.12} ; A_{2.1}\right) \cup((D-) 2)
$$$$
\text { [b,ezúmn-ij] (15) - [b,ezúm-stvova-t, ] }
$$

(S/stvova/; $\left.M_{1.12} ; A_{2.1}\right) u(D-V / 5)$

[blagodárn-ij] (40)-

[blagodár-stvova-t,]

$$
\begin{aligned}
& \text { (S/stvova/; } \left.M_{1.12 u 3.1} ; A_{2.1}\right) u(D-V / 4) \\
& \text { [blagodúsn-ij] ( } 4)- \\
& \text { [blagodúse-stvova-t, ] (-) }
\end{aligned}
$$

Anzahl der tatsächlich realisierten Kombinationen: $\underline{3}$

Anzahl der theoretisch maximal möglichen Kombinationen: 4

- Zusätzlich zu den realisierten ist die Kombination ( 5 /stroval; $M_{3.1} ; A_{2.1}$ ) theoretisch möglich. 
Anzahl der theoretisch minimal möqlichen Kombinationen: 1

- Theoretisch möglich ist ein Wegfall der Kombinationen (S/stvova/; $A_{2.1}$ ) und (S/stvova/; $M_{1.12} ; A_{2.1}$ ).

Somit verwirklicht das Russische zur Bildung von Verben mit der allgemeinen Explikatoon 'Das mit dem ableitenden Adjektiv benannte Merkmal annehmen oder an den Tag legen'

$$
4+7+7+3+2+5+5+3=36
$$

unterschiedliche Kombinationen der zur Verfügung stehenden Ausdrucksmittel.

Theoretisch maximal möglich sind

$$
4+13+23+9+3+7+6+4=69
$$

unterschiedliche Kombinationen.

Theoretisch minimal möglich sind

$$
3+6+4+3+2+4+3+1=26
$$

unterschiedliche Kombinationen dieser Ausdrucksmittel.

2.4.4.2. Etwas (jdn.) mit dem durch das ableitende Adjektiv benannte Merkmal versehen

2.4.4.2.0.1. Aufzählung der Derivatemtypen sowie der jeweils miteinander kompatiblen Ausdrucksmittel zur Bildung von Verben mit dieser allgemeinen Explikation

2.4 .4 .2 .0 .1 .1 . D-I, D-II/I, D-III/7, D-V/4

$$
\begin{aligned}
& \mathrm{S} / \mathrm{i}_{2} / \\
& M_{1.11} \text { aber }[m, e ́ l k,-i j] \underset{[m, e 1, \dot{c},-1-t,](1)}{(201)-}
\end{aligned}
$$

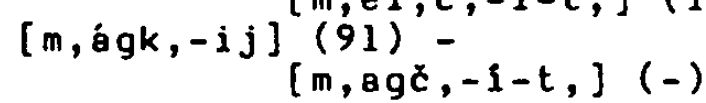

$$
\begin{aligned}
& \left.M_{1.12} \text { aber }[t, o m n-i j]{ }_{[}^{(223)-},-i m n,-i-t,\right] \text { (2) } \\
& \text { [bl,édn-ij] (45) - } \\
& {[b l, e d n,-i-t,](-)} \\
& \begin{array}{r}
{[g r, a z n-i j]} \\
{[g r, a z n,-i-t,](-)}
\end{array} \\
& \text { [póln-ij] (sls) - } \\
& \text { poln,-i-t, ] (-) } \\
& \begin{aligned}
& {[p l o ́ t n-i j] }(46)- \\
& {[p l o t n,-i-t,](-) }
\end{aligned} \\
& {[\grave{c}, \dot{o r n}-\mathbf{i j}](473)-} \\
& \text { [ć, orn, }-i-t,] \text { (1) }
\end{aligned}
$$

M. 13 


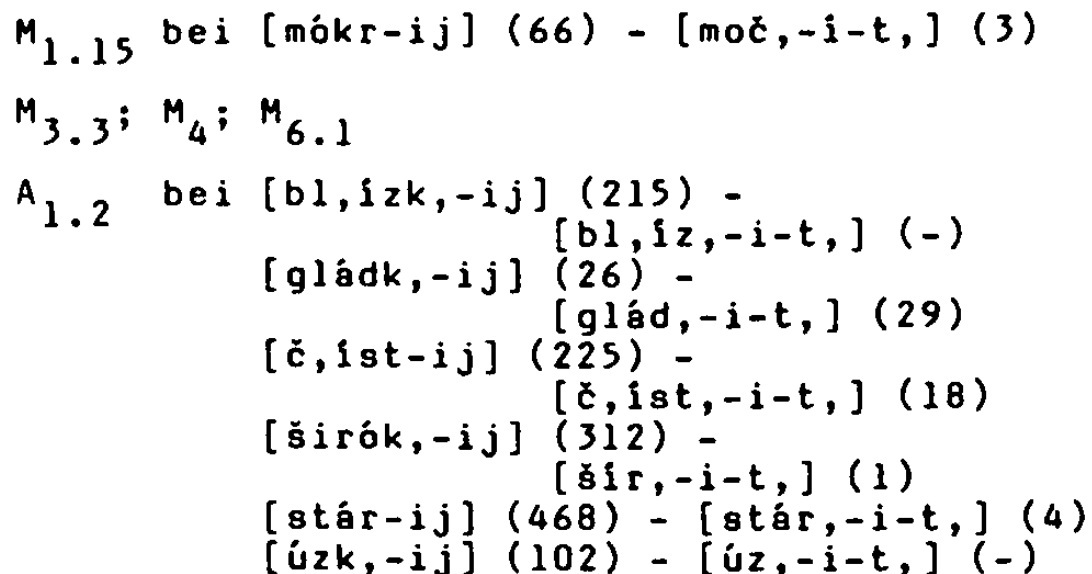

$A_{2.2}, A_{2.4}$ sonst

$2 \cdot 4 \cdot 4 \cdot 2 \cdot 0.1 \cdot 2 . \quad D-1$

$\mathrm{SuPo} / \mathrm{i} \ldots \mathrm{s,a/2}$

$M_{1.12} ; M_{4}$

$A_{2.2}, A_{2.4}$

$2 \cdot 4 \cdot 4 \cdot 2 \cdot 0.1 \cdot 3 . D-1,0-11 / 1$

S/ovar

$M_{1.1}$ bei [opt,imál, n-ij] $(12)-$
$[o p t, i m, i z, i r-o v a-t],(-)$

$M_{1.6}, M_{1.7}, M_{1.9}, M_{1.12}, M_{1.16}$

$M_{2.11}, M_{2.12}, M_{2.13}$

$\mathrm{M}_{4}$

$A_{1.2}$ wenn $M_{2.11}, M_{2.13}$

$A_{1.3 .2}$ wenn $M_{2.12}$

$2.4 \cdot 4 \cdot 2 \cdot 0 \cdot 1.4 . \quad D-1$

Pus/vi ...i i/

$M_{1.12}$ bei [jávn-ij] (33)-[vi-jav,-i-t,] (7)

$\mathrm{M}_{4}$

A.1

$2.4 .4 .2 .0 .1 .5 . \quad D-1, D-11 / 1$

PuS/za ... il

$M_{1.11}$ 


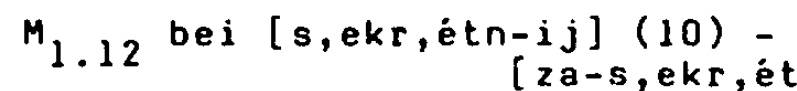

$[z a-s, e k r, e t,-i-t$,$] (1)$

$M_{1.13} ; M_{4}$

$A_{2.2}, A_{2.4}$

$2.4 \cdot 4 \cdot 2 \cdot 0.1 \cdot 6.0-1$

PuS/na ... il

$M_{4}$

$A_{1.2}$

2.4 .4 .2 .0 .1 .7$. D-I, D-II/I, D-V/S

PuS/o ... il

M.11

$M_{1.12}$ bei [blagoródn-ij] (58) -
$[0-b$ lagoród,-i-t,] (-)

$M_{4}$

$A_{2.2}, A_{2.4}$

$2 \cdot 4 \cdot 4 \cdot 2 \cdot 0.1 .8 . \quad$ D-I

PuS/ob ... il

$M_{1.11}$ bei [korótk, $\left.-i j\right](91)-$

$M_{4} ; M_{6.1}$

$A_{2.4}$

$2.4 \cdot 4 \cdot 2 \cdot 0.1 .9 . \quad 0-I$

PuS/pro .... i/

$M_{1.11} ; M_{4}$

$A_{2.4}$

$2 \cdot 4 \cdot 4 \cdot 2 \cdot 0 \cdot 1 \cdot 10 \cdot 0-1$

$$
\begin{aligned}
& \mathrm{PuS}_{\text {raz }} \ldots \mathrm{i} / \mathrm{V} / \mathrm{ras} \ldots \mathrm{i} / \mathrm{I} \\
& \mathrm{M}_{1.11}, \mathrm{M}_{4} \\
& \mathrm{~A}_{2.4}
\end{aligned}
$$

$2 \cdot 4 \cdot 4 \cdot 2 \cdot 0.1 .11 .0-1,0-11 / 1$ 


$$
\begin{aligned}
& P_{1 / s} \ldots i \text { i/ } \\
& M_{1.11}, M_{1.12} ; M_{4} \\
& A_{1.2} \text { bei [dóbr-ij] }(277)- \\
& A_{2.2}, A_{2.4} \text { sonst }
\end{aligned}
$$

$2 \cdot 4 \cdot 4 \cdot 2 \cdot 0 \cdot 1 \cdot 12 . D-I, D-I I / 1$

PuS/u ... il

$M_{1.11}, M_{1.13} ; M_{4}$

$\mathrm{A}_{2.2}, \mathrm{~A}_{2.4}$

$2 \cdot 4 \cdot 4 \cdot 2 \cdot 0 \cdot 1 \cdot 13 \cdot 0-1$

PuS/za $\ldots$ el

$M_{4} ; M_{6.1}$

$A_{2,1}$

$2.4 .4 .2 .0 .1 .14, D-I, D-I I / 2, D-V / 4$

PuS/o ...e e/

$\mathrm{M}_{1.12} ; \mathrm{M}_{4}$

$A_{2.2}, A_{2.4}$

$2 \cdot 4 \cdot 4 \cdot 2 \cdot 0 \cdot 1 \cdot 15 \cdot 0-1$

Pus/po ....el

$\mathrm{M}_{4}$

$A_{2.2}$

2.4.4.2.0.2. Die Kombinatorik dieser Mittel

$$
\begin{aligned}
& 2 \cdot 4 \cdot 4 \cdot 2 \cdot 0 \cdot 2 \cdot 1 . \quad\left(S_{/ i_{2} /} ; M_{1.11 \cup 4} ; A_{1.2}\right) \cup(D-I) \\
& {[b 1, i z k,-i j](215)-[b 1, i z,-i-t,](-)} \\
& \left(\mathrm{S} / \mathrm{i}_{2} / \mathrm{M}_{1.11 \cup 4} ; \mathrm{A}_{2.4}\right) u(\mathrm{D}-\mathrm{I}) \\
& {[k r, e ́ p k,-i j](70)-[k r, e p,-i-t,](7)} \\
& \left(\mathrm{S} / \mathrm{i}_{2} / \mathrm{M}_{4 \cup 6.1} ; \mathrm{A}_{2.4}\right) \cup(\mathrm{D}-\mathrm{I}) \\
& {[m, e ́ 1 k,-i j](201)-[m, e l, \grave{c},-i-t,](1)}
\end{aligned}
$$




$$
\begin{aligned}
& \left(\mathrm{S} / \mathrm{i}_{2} / \mathrm{M}_{6.1} ; \mathrm{A}_{2.4}\right) \cup(\mathrm{D}-\mathrm{I}) \\
& {[m, a ́ g k,-i j](91)-[m, a g c ̌,-i-t,](-)} \\
& \left(S_{/ i_{2}} / M_{1.12 \cup 4} ; A_{2.2}\right) \cup(D-V / 4) \\
& \text { [raznoobrázn-ij] (132) - } \\
& {[\text { raznoobráz, }-i-t,](-)} \\
& \left(\mathrm{S} / \mathrm{i}_{2} / \mathrm{M}_{1.12 \cup 4} ; \mathrm{A}_{2.4}\right) \cup(D-1) \\
& \text { [mútn-ij] (27) }-[m u t,-i-t,](7) \\
& \left(\mathrm{S} / \mathrm{i}_{2} / ; \mathrm{M}_{1.13 \cup 4} ; \mathrm{A}_{1.2}\right) \cup(\mathrm{D}-\mathrm{I}) \\
& {[\text { śrók, -ij] (312) - [\$ir,-i-t, ] (1) }}
\end{aligned}
$$

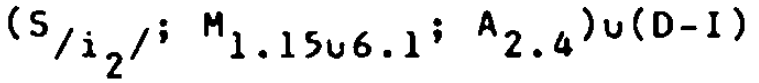

$$
\begin{aligned}
& {[\text { mókr-ij] (66) }-[\operatorname{moc},-i-t,] \text { (3) }} \\
& \left(S / i_{2} / M_{3.3 U 4} ; A_{2.4}\right) \cup(D-I I / 1) \\
& {[1, e d, a n-o j](48)-[1, e d, e n,-i-t,](2)} \\
& \left(S / i_{2} / M_{4} ; A_{1.2}\right) \cup(D-1) \\
& \text { [stár-ij] (468)-[stár,-i-t,] (4) } \\
& \left(S / i_{2} f ; M_{4} ; A_{2.2}\right) \cup(D-I) \\
& {[f a l, \grave{s} i v-i j](10)-[f a l, \grave{s} i v,-i-t,](-)} \\
& \left(S / i_{2} / M_{4} ; A_{2.4}\right) \cup(D-I) \\
& {[b l, e ́ d n-i j](45)-[b l, e a n,-i-t,](-)} \\
& \left(\mathrm{S} / \mathrm{i}_{2} / \mathrm{M}_{4} ; \mathrm{A}_{2.4}\right) \cup(\mathrm{D}-\mathrm{III} / 7) \\
& {[p, a ́ n-i j](88)-[p, a n,-i-t,]}
\end{aligned}
$$

Anzahl der tatsächlich realisierten Kombinationen: 12

Anzahl der theoretisch maximal möglichen Kombinationen: 29

- Zusätzlich zu den realisierten sind die Kombinationen

$\left(S / i_{2} / ; A_{1.2}\right),\left(S / i_{2} / A_{2.2}\right),\left(S / i_{2} / A_{2.4}\right) ;\left(S / i_{2} / ;\right.$

$\left.M_{1.11} ; A_{1.2}\right),\left(S / i_{2} / M_{1.11} ; A_{2.2}\right),\left(S / i_{2} / M_{1.11} ; A_{2.4}\right) ;$

$\left(S / i_{2} / M_{1.11 \cup 4} ; A_{2.2}\right) ;\left(S / i_{2} / M_{1.12} ; A_{1.2}\right),\left(S / i_{2}\right)^{;}$

$\left.M_{1.12} ; A_{2.2}\right),\left(S / i_{2} / M_{1.12} ; A_{2.4}\right) ;\left(S / i_{2} ; M_{1.1204}:\right.$

$\left.A_{1.2}\right) ;\left(S / i_{2} / M_{1.13 \cup 4} ; A_{2.2}\right),\left(S / i_{2} / M_{1.13 \cup 4} ; A_{2.6}\right) ;$

$\left(S / i_{2} / M_{3.304} ; A_{1.2}\right),\left(S / i_{2} / M_{3.304} ; A_{2.2}\right) ;\left(S / i_{2}\right.$; 
$\left.M_{6.1} ; A_{1.2}\right) ;\left(S / i_{2} ; M_{6.1} ; A_{2.2}\right)$ theoretisch möglich.

Anzahl der theoretisch minimal möglichen Kombinationen: $\underline{8}$

- Theoretisch möglich ist ein Wegfall der Kombinationen $\left(S / i_{2} / M_{4} ; A_{1.2}\right),\left(S / i_{2} / M_{4} ; A_{2.2}\right),\left(S / i_{2} / M_{4} ; A_{2.4}\right)$ sowie $\left(S / i_{2} / M_{6.1} ; A_{2.2}\right)$.

$2.4 .4 .2 .0 .2 .2 . \quad\left(S \cup P_{0} / i \ldots 8,8 / 2 ; M_{1.12 \cup 4} ; A_{2.2}\right) \cup(D-I)$

$$
\begin{aligned}
& \text { [zemánn-ij] (-) - [zemán,-i-t,-s,a] (-) } \\
& \left(S \cup P O / i \ldots 8, a / 2 ; M_{4} ; A_{2.2}\right) \cup(D-1) \\
& {[u g r, u m-i j](21)-[u g r, u m,-i-t,-s, 0](-)} \\
& \left(S \cup P o / i \ldots 8,8 / 2 ; M_{4} ; A_{2.4}\right) \cup(D-I) \\
& \text { [skup-ój] (17) - [skup, -i-t,-s,a] (4) }
\end{aligned}
$$

Anzahl der tatsächlich realisierten Kombinationen: $\underline{3}$ Anzahl der theoretisch maximal möqlichen Kombinationen: $\underline{8}$

- Zusätzlich zu den realisierten sind die Kombinationen $\left(S_{P O} / i \ldots s, a / 2 ; A_{2.2}\right),\left(S_{2} P_{0} / i \ldots s, a / 2 ; A_{2.4}\right) ;$

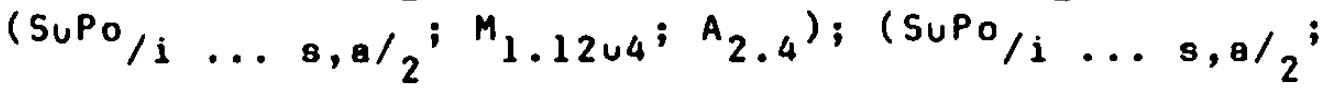
$\left.M_{1.12} ; A_{2.2}\right)$ und $\left(S u P o / i \ldots 8,8 / 2 ; M_{1.12} ; A_{2.4}\right)$ theoretisch möglich.

Anzahl der theoretisch minimal möglichen Kombinationen: $\underline{2}$

- Theoretisch möglich ist ein Wegfall der Kombination $\left(S_{4} P_{/ i} \ldots s, 8 / 2 ; M_{4} ; A_{2.2}\right)$.

$$
\begin{aligned}
& 2.4 .4 .2 .0 .2 .3 . \quad\left(5 / \text { ova }_{2} / M_{\left.1.1 \cup 2.13 \cup 4 ; A_{1.2}\right) \cup(D-1)}\right. \\
& \text { [opt,imál,n-ij] } \underset{[o p t, i m, i z, i r-o v a-t,](-)}{(12)-} \\
& \left(S_{\text {/ova }} / \mathrm{M}_{\left.1.6 U 2.13 ; \mathrm{A}_{1.2}\right) \cup(D-I I / 1)}\right. \\
& \text { [mat, emat, ić,esk, -ij] (13) - } \\
& \text { [mat, emat,iz,ir-ova-t,] (-) } \\
& \left(S_{\text {/ova }} / ; M_{\left.1.7 \cup 2.13 ; A_{1.2}\right) \cup(D-I I / 1)}\right. \\
& {[r, i t m, i c ̌ n-i j] \underset{[r, i t m, i z, i r-o v a-t,](-)}{(3)-}}
\end{aligned}
$$




$$
\begin{aligned}
& \left(\mathrm{S} / \mathrm{ova}_{2} / \mathrm{M}_{1.9} \mathrm{C}_{2.13} ; \mathrm{A}_{1.2}\right) \cup(D-11 / 1) \\
& \begin{array}{l}
\text { [fašistsk,-ij] }(60)- \\
{[\text { fašiz, ir-ova-t, ] (-) }}
\end{array} \\
& \text { (S/ova } \left.{ }^{\prime} ; M_{1.12 \cup 2.11 \cup 4} ; A_{1.2}\right) \cup(D-1) \\
& \text { [ob-ekt, ivn,-ij] (57) } \\
& \text { [ob-ekt, iv, ir-ova-t, ] (-) } \\
& \left(\mathrm{S} / \mathrm{ova}_{2} / \mathrm{M}_{\left.1.12 \cup 2.12 ; A_{1.3 .2}\right) \cup(D-I)}\right. \\
& {[n, e j t r a ́ l, n-i j](16)-} \\
& {[n, e j t r a l, i z-o v a-t,](1)} \\
& \left(\mathrm{S} / \mathrm{ova}_{2} / \mathrm{M}_{\left.1.16 \cup 2.13 \cup 4 ; A_{1.2}\right) \cup(D-1)}\right. \\
& {[g, e r m a ́ n s k,-i j](116) \text { - }} \\
& \{g, \text { erman,iz,ir-ova-t, }\}(-)
\end{aligned}
$$

Anzahl der tatsächlich realisierten Kombinationen: $?$

Anzahl der theoretisch maximal möglichen Kombinationen: 20

- Zusätzlich zu den realisierten sind die Kombinationen

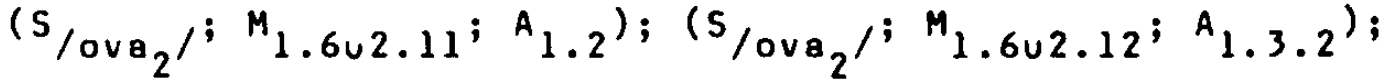

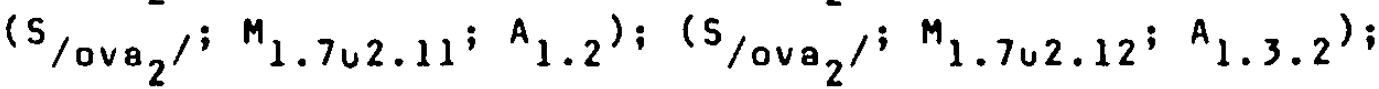

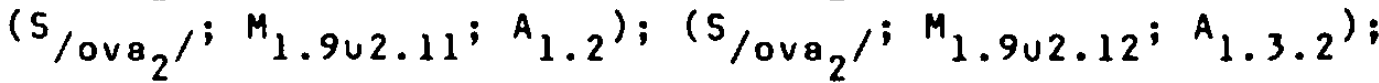

$$
\begin{aligned}
& \left(S_{\text {/ova }} / \mathrm{M}_{1.12 \cup 2.11} ; \mathrm{A}_{1.2}\right) ;\left(\mathrm{S}_{\text {/ova }} / \mathrm{B}_{1.1202 .13} ; \mathrm{A}_{1.2}\right) \text {; }
\end{aligned}
$$

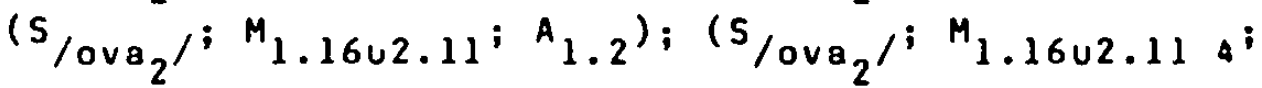

$$
\begin{aligned}
& \left.A_{1.2}\right) ;\left(S_{/ o v a} / \mathrm{M}_{1.1602 .12} ; A_{1.3 .2}\right) ;\left(5 / o v a_{2} / ;\right.
\end{aligned}
$$

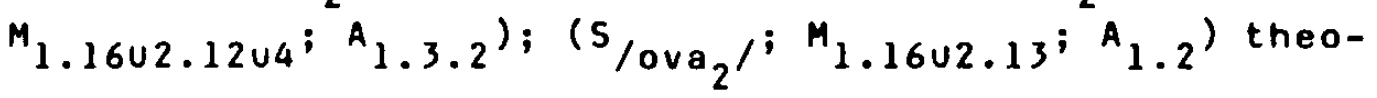

Anzahl der theoretisch minimal möglichen Kombinationen: ?

$2.4 \cdot 4.2 .0 .2 .4 . \quad\left(P u S / v i \ldots i j ; M_{1.12 v 4} ; A_{1.1}\right) \cup(D-1)$

$$
\begin{gathered}
{[j a \cup n-i j](33)-[v i-j a v,-i-t,] \text { (7) }} \\
\left(P \cup S / v i \ldots j / M_{4} ; A_{1,1}\right) \cup(D-I) \\
{[j a \operatorname{sn-ij]}(146)-[v i-j a 8 n,-i-t,] \text { (47) }}
\end{gathered}
$$

Anzahl der tatsächlich realisierten Kombinationen: $\underline{2}$

Anzahl der theoretisch maximal möglichen Kombinationen: $\underline{4}$

- Zusätzlich zu den realisierten sind die Kombinationen $\left(P u S / v i \ldots i / ; A_{1.1}\right)$ sowie (PuS/vi ... i/; $M_{1.12} ; A_{1.1}$ ) 
theoretisch möglich.

Anzahl der theoretisch minimal möglichen Kombinationen: $\underline{1}$

- Theoretisch möglich ist ein Wegfall der Kombination $\left(P \cup S / v i \ldots i / M_{4} ; A_{1.1}\right)$.

$2.4 .4 .2 .0 .2 .5 . \quad(P \cup S / z a \ldots i) ; M_{\left.1.11 \cup 4 ; A_{2.4}\right) \cup(D-I)}$ $[n, i z \hat{k},-i j](116)-[z a-n, i z,-i-t],(-)$

$\left(P_{u} / 2 a \ldots i / M_{1.12 \cup 4} ; A_{2.2}\right) \cup(D-11 / 1)$ [s,ekr,étn-ij] (10) $[z a-s, e k r, e ́ t,-i-t],(-)$

(PuS/za ... i/; $M_{\left.1.13 \cup 4 ; A_{2.2}\right) \cup(D-I)}$ $[v i s o \dot{k},-i j](549)-[2 a-v i s,-i-t],(-)$

$\left(P \cup S / z a \ldots i / M_{4} ; A_{2.4}\right) \cup(D-I)$

[trúdn-ij] (143)-[2a-trudn,-i-t, ]

Anzahl der tatsächlich realisierten Kombinationen: 4

Anzahl der theoretisch maximal möglichen Kombinationen: 14

- Zusätzlich zu den realisierten sind die Kombinationen

$$
\begin{aligned}
& \left(P \cup S / z a \ldots i / A_{2.2}\right),\left(P \cup S / z a \ldots i / A_{2.4}\right) \text {; } \\
& \left(P \cup S / z a \ldots i / M_{1.11 \cup 4} ; A_{2.2}\right) ;(P \cup S / 2 a \ldots i) ; M_{1.11} ; \\
& \left.\left.A_{2.2}\right) ;(P \cup S / 2 a \ldots i) ; M_{1.11} ; A_{2.4}\right) ;(P \cup S / z a \ldots i \text {; } \\
& \left.M_{1.12} ; A_{2.2}\right),\left(P \cup S / 2 a \ldots i / M_{1.12} ; A_{2.4}\right) ; \\
& \text { (PuS/za ... i); } \left.M_{1.12 \cup 4} ; A_{2.4}\right) ;(P \cup S / 2 a \ldots i) ; \\
& \left.M_{1.13 \cup 4} ; A_{2.4}\right) ;\left(P \cup S / 2 a \ldots i / M_{4} ; A_{2.2}\right) \text { theoretisch } \\
& \text { möglich. }
\end{aligned}
$$

Anzahl der theoretisch minimal möglichen Kombinationen: $\underline{3}$

- Theoretisch möglich ist ein Wegfall der Kombination $\left(P \cup S / 28 \ldots i / M_{4} ; A_{2.4}\right)$.

$\left.2.4 .4 .2 .0 .2 .6 . \quad(P \cup S / n a \ldots i) ; M_{4} ; A_{1.2}\right) \cup(D-I)$

[póln-ij] (515)-[na-póln,-i-t,] (40)

$\left(P u S / n a \ldots i / M_{4} ; A_{1.2}\right) \cup(D-I)$

$[8 i t-i j](25)-[n \theta-8 i t,-i-t$,

Anzahl der tatsächlich realisierten Kombinationen: 1

Anzahl der theoretisch maximal möglichen Kombinationen: $\underline{2}$

- Zusätzlich zu der realisierten ist die Kombination (PuS/na ... i/; $A_{1.2}$ ) theoretisch möglich.

Anzahl der theoretisch minimal möglichen Kombinationen: 1 
$2 \cdot 4 \cdot 4 \cdot 2 \cdot 0.2 .7 . \quad\left(P \cup S / 0 \ldots i\right.$ i $\left.; M_{1.11 \cup 4} ; A_{2.4}\right) \cup(D-1)$

$$
\begin{aligned}
& \text { [korótk,-ij] }(91)-[0-k \operatorname{orot},-i-t,](-) \\
& \left(P \cup S / 0 \ldots \text { i } ; M_{\left.1.12 \cup 4 ; A_{2.2}\right) \cup(D-V / S)}\right. \\
& \text { [blagoródn-ij] (58) } \\
& \text { [o-b]agoród, }-i-t,](-) \\
& \left(P \cup S / 0 \ldots i / ; M_{4} ; A_{2.2}\right) \cup(D-I) \\
& \text { [kurguiz-ij] (2) - [o-kurgúz, -i-t,] (-) } \\
& \left(P \cup S / 0 \ldots i / ; M_{4} ; A_{2.4}\right) \cup(D-I) \\
& \text { [slóżn-ij] (239) - [0-složn,-i-t,] (1) } \\
& \text { (PuS/0 ... i /; } \left.M_{4} ; A_{2.4}\right) \cup(D-I I / 1) \\
& \text { [krúgi-ij] (88)-[0-krugl, }-i-t,](-)
\end{aligned}
$$

Anzahl der tatsächlich realisierten Kombinationen: 4

Anzahl der theoretisch maximal möqlichen Kombinationen: 12

- Zusätzlich zu den realisierten sind die Kombinationen

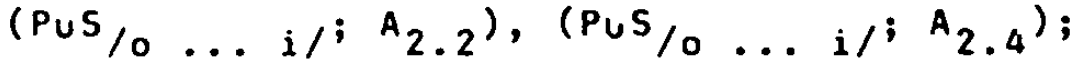

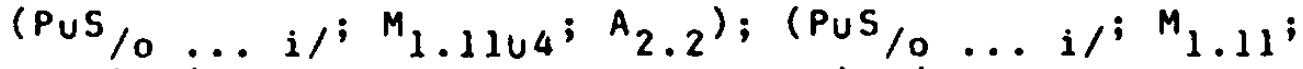

$$
\begin{aligned}
& \left.\left.A_{2.2}\right) \text {, (PuS/0,.. i/; } M_{1.11} ; A_{2.4}\right) ;(P \cup S / 0 \ldots i / ; \\
& \left.M_{1.12 \cup 4} ; A_{2.4}\right) ;\left(P \cup S / 0 \ldots i / M_{1.12} ; A_{2.2}\right),(P \cup S / 0 \ldots i / ; \\
& M_{1.12} ; A_{2.4} \text { ) theoretisch möglich. }
\end{aligned}
$$

Anzahl der theoretisch minimal möglichen Kombinationen: $\underline{2}$

- Ein Wegfall der Kombinationen (PuS/0 ... i/; $M_{4} ; A_{2.2}$ ) und (PuS/0 ... i/ $; M_{4} ; A_{2.4}$ ) ist theoretisch möglich.

$2 \cdot 4 \cdot 4 \cdot 2 \cdot 0.2 .8 . \quad\left(P \cup S / 0 b \ldots i / ; A_{2.4}\right) \cup(D-1)$

[óbšc $\ddot{c},-i j](401)-[o b-o b s ̌ c ̆,-i-t$,

$$
\text { (PuS/ob ... i/; } M_{\left.1.11 \cup 4 ; A_{2.4}\right) \cup(D-I)}
$$

[korótk, $-i j](91)-[o b-k o r o t,-i-t],(-)$

$$
\begin{aligned}
& \left(P \cup S / o b \ldots ; j ; M_{4} ; A_{2.4}\right) \cup(D-I) \\
& {[\text { nov-ij] }(1722)-[o b-n o v,-i-t,]}
\end{aligned}
$$

(PuS/ob ... i/; $\left.M_{6.1} ; A_{2.4}\right) \cup(D-1)$

$[1, o g k,-i j](152)-[o b-1, o g c ́,-i-t$,

Anzahl der tatgächlich realisierten Kombinationen: 4

Anzahl der theoretisch maximal möglichen Kombinationen: $\underline{5}$

- Zusätzlich zu den realisierten ist die Kombination (PuS/ob ... i/ ; $M_{1.11} ; A_{2.4}$ ) theoretisch möglich.

Anzahl der theoretisch minimal möglichen Kombinationen: $\underline{2}$ 
- Theoretisch möglich ist ein Wegfall der Kombinationen (PuS/ob ... i/ ; $A_{2.4}$ ) sowie (PuS/ob ... i/; $M_{4} ; A_{2.4}$ ).

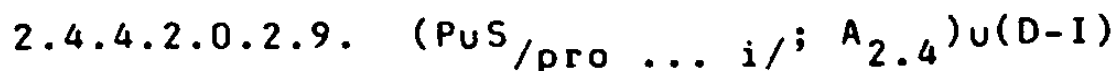

$$
\begin{aligned}
& {[s v, \dot{e} z-i j](84)-[\text { pro-sv,e } z-i-t,](-)} \\
& \text { (PuS/pro ... i } / M_{1.11 \cup 4} ; A_{2.4} \text { ) } \cup(D-1) \\
& {[r, \dot{e} d k,-i j](80)-[p r o-r, e d,-i-t,](-)} \\
& \text { (PuS/pro ... i/; } \left.M_{4} ; A_{2.4}\right) \cup(D-I) \\
& {[j a s n-i j](146)-[\text { pro-jasn, -i-t, ] }}
\end{aligned}
$$

Anzahl der tatsächlich realisierten Kombinationen: $\underline{3}$

Anzahl der theoretisch maximal möqlichen Kombinationen: 4

- Zusätzlich zu den realisierten ist die Kombination (PuS/pro ... il/ $M_{1.11} ; A_{2.4}$ ) theoretisch möglich.

Anzahl der theoretisch minimal möqlichen Kombinationen: 1

- Theoretisch möglich ist ein Wegfall der Kombinationen

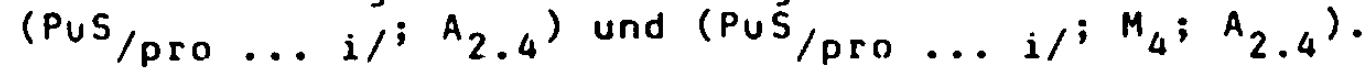

$2.4 .4 .2 .0 .2 .10 .\left(P \cup S / r a z \ldots i / 1 ; M_{1.11 \cup 4} ; A_{2.4}\right) u(D-I)$

$$
\begin{aligned}
& {[r, \dot{e d k},-i j](80)-[r a z-r, e d,-i-t,](-)} \\
& \left(P \cup S / r a s \ldots i / 1 ; M_{4} ; A_{2.4}\right) \cup(D-I) \\
& [s] a b-i j](98)-[\text { ras-s }) a b,-i-t,](-)
\end{aligned}
$$

Anzahl der tatsächlich realisierten Kombinationen: $\underline{2}$

Anzahl der theoretisch maximal möglichen Kombinationen: 4

- Zusätzlich zu den realisierten sind die Kombinationen (Pus/raz ... i/ v/ras ... i/,; $A_{2.4}$ ) sowie

(Pus/raz ... i/ v/ras ... i/ 1 ; $M_{1.11 ;} A_{2.4}$ ) theoretisch möglich.

Anzahl der theoretisch minimal möglichen Kombinationen: 1

- Theoretisch möglich ist ein Wegfall der Kombination $\left(\right.$ Pus/raz ... i/ v/ras ... i/,; $\left.M_{4} ; A_{2.4}\right)$.

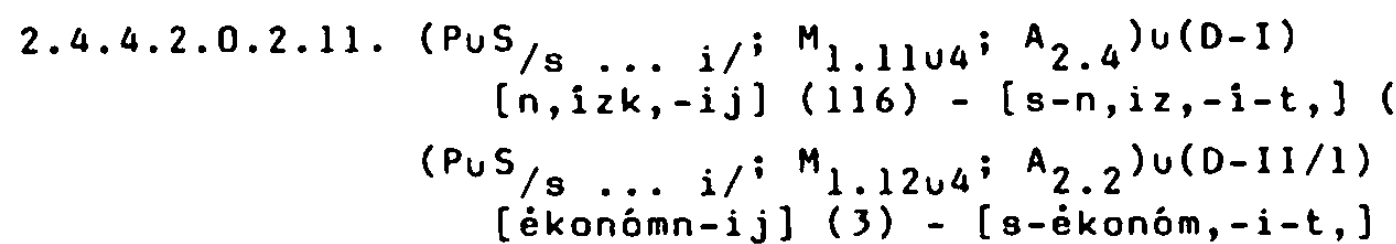




$$
\begin{aligned}
& \left(P_{u} / s \ldots i / ; M_{4} ; A_{1.2}\right) \cup(D-I) \\
& \text { [dóbr-ij] (277) - [s-dóbr, -i-t,] (1) } \\
& \left(P \cup S / s \ldots i / M_{4} ; A_{2.4}\right) \cup(D-11 / 1) \\
& \text { [krúgl-ij] (88)-[s-krugl, -i-t,] (-) }
\end{aligned}
$$

Anzahl der tatsächlich realisierten Kombinationen: 4

Anzahl der theoretisch maximal möglichen Kombinationen: 18

- Zusätzlich zu den realisierten sind die Kombinationen

$$
\begin{aligned}
& \left(P \cup S / s \ldots i / A_{1.2}\right),\left(P \cup S / s \ldots i / ; A_{2.2}\right) \text {, } \\
& \left(P \cup S / s \ldots \text { i } ; A_{2.4}\right) ;\left(P \cup S / s \ldots \text { i } ; M_{1.11} ; A_{1.2}\right) \text {, } \\
& \left(P \cup S / s \ldots i \text { i } M_{1.11} ; A_{2.2}\right),\left(P_{0 S} / s \ldots i / M_{1.11} ; A_{2.4}\right) \text {; } \\
& \left.(P \cup S / s \ldots i) ; M_{1.11 \cup 4} ; A_{1.2}\right),\left(P \cup S / s \ldots i / M_{1.11 \cup 4} ;\right. \\
& \left.A_{2.2}\right) ;\left(P \cup S / s \ldots i \text {; } M_{1.12} ; A_{1.2}\right),\left(P \cup S / s \ldots i / M_{1.12} ;\right. \\
& \left.A_{2.2}\right),\left(P \cup S / s \ldots i \text { i } ; M_{1.12} ; A_{2.4}\right) ;(P \cup S / s \ldots i \text {; }
\end{aligned}
$$

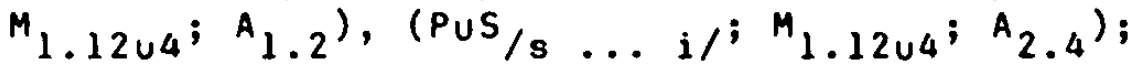

$$
\begin{aligned}
& \text { ( } P \cup S / s \ldots \text { i } / M_{4} ; A_{2.2} \text { ) theoretisch möglich. }
\end{aligned}
$$

Anzahl der theoretisch minimal möglichen Kombinationen: $\underline{3}$

- Theoretisch möglich ist ein Wegfall der Kombination $\left(P_{\text {uS }} / \mathrm{s} \ldots \mathrm{i} / \mathrm{M}_{4} ; \mathrm{A}_{2.4}\right)$.

$2 \cdot 4 \cdot 4 \cdot 2 \cdot 0.2 .12 .\left(P_{u S} / u \ldots . i j ; A_{2.2}\right) u(D-1)$

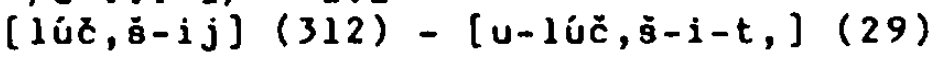

$$
\left(P_{u S} / u \ldots i / M_{1.11 \cup 4} ; A_{2.4}\right) \cup(D-I)
$$

[korotk, $-i j](91)-[u-k o r o t,-i-t],(-)$

$$
\left(P_{u S} / u \ldots i \text { il; } M_{\left.1.13 u 4 ; A_{2.4}\right) \cup(D-I)}\right.
$$

[glubók, -ij] (216)-[u-glub,-i-t,] (4)

$$
\begin{array}{r}
\left(P \cup S / u, \ldots i / M_{4} ; A_{2,2}\right) \cup(D-1 I / 1) \\
{[m, i \text { iost,iv-ij] }(1 i)-} \\
{[u-m, i l o s t, i v,-i-t,]}
\end{array}
$$

$$
\begin{aligned}
& \left(P \cup S / u, \ldots i / ; M_{4} ; A_{2,4}\right) \cup(D-1) \\
& \quad[v] a z i n-i j](24)-[u-v l a z i n,-i-t,]
\end{aligned}
$$

Anzahl der tatsächlich realisierten Kombinationen: $\underline{5}$

Anzahl der theoretisch maximal möglichen Kombinationen: 12

- Zusätzlich zu den realisierten sind die Kombinationen

$$
\begin{aligned}
& \left(P \cup S / u \ldots \text { i } / A_{2.4}\right) ;\left(P \cup S / u \ldots i \text { i } ; M_{1.11 ;} A_{2.2}\right. \text { ), } \\
& \left(P \cup S / u \ldots \text { i); } M_{1.11} ; A_{2.4}\right) ;(P \cup S / u \ldots i) ; M_{1.11 \cup 4} \text {; } \\
& \left.A_{2.2}\right) ;\left(P \cup S / u \ldots i \text {; } M_{1.13} ; A_{2.2}\right),(P \cup S / u \ldots i) ; \\
& \left.M_{1.13} ; A_{2.4}\right) ;\left(P \cup S / u \ldots i / M_{1.13 \cup 4} ; A_{2.2}\right) \text { theoretisch }
\end{aligned}
$$


möglich.

Anzahl der theoretisch minimal möglichen Kombinationen: $\underline{3}$

- Theoretisch möglich ist ein Wegfall der Kombinationen $\left(P \cup S / u \ldots i / A_{2.2}\right)$ sowie $\left(P \cup S / u \ldots i / ; M_{4} ; A_{2.4}\right)$.

$2 \cdot 4 \cdot 4 \cdot 2 \cdot 0.2 .13 .\left(P u S / z a \ldots e / ; M_{4} ; A_{2.1}\right) \cup(D-1)$

[kor, ić,n,ev-ij] (23)-

$[z a-k o r, i c, n, e v,-e-t],(-)$

$\left(P \cup S / z a \ldots e / ; M_{6.1} ; A_{2.1}\right) \cup(D-I)$

$[t, i x,-i j](120)-[z a-t, i s-e-t],(-)$

Anzahl der tatsächlich realisierten Kombinationen: $\underline{2}$

Anzahl der theoretisch maximal möglichen Kombinationen: $\underline{3}$

- Zusätzlich zu den realisierten ist die Kombination $\left(P u S / z a \ldots e / A_{2.1}\right)$ theoretisch möglich.

Anzahl der theoretisch minimal möglichen Kombinationen: $\underline{2}$

$2.4 .4 .2 \cdot 0.2 .14 .\left(P_{0} / 0 \ldots e / ; M_{1.12 \cup 4} ; A_{2.2}\right) \cup(D-V / 4)$

[poloúmn-ij] (4)-[o-poloúm,-e-t,] (2)

(PuS/0 ...e e/ $\left.M_{4} ; A_{2.4}\right) \cup(D-I)$

[gól-ij] (73)-[o-gol, -é-t,] (-)

(PuS/0 ...e e; $\left.M_{4} ; A_{2.4}\right) \cup(D-11 / 2)$

[rogov-ój] (5) - [o-rogov, -é-t, ] (-)

Anzahl der tatsächlich realisierten Kombinationen: 2

Anzahl der theoretisch maximal möqlichen Kombinationen: $\underline{8}$

- Zusätzlich zu den realisierten sind die Kombinationen
$\left(P \cup S / 0 \ldots\right.$ e/; $\left.A_{2.2}\right),\left(P \cup S / 0 \ldots e / A_{2.4}\right) ;$
$\left(P_{\cup} / 0 \ldots\right.$ e); $\left.M_{1.12} ; A_{2.2}\right)$, (PuS /o .. e/; $\left.M_{1.12} ; A_{2.4}\right)$,
$\left(P \cup S / 0 \ldots\right.$ e/; $\left.M_{1.12 \cup 4} ; A_{2.4}\right) ;\left(P \cup S / 0 \ldots e / ; M_{4} ; A_{2.2}\right)$
theoretisch möglich.

Anzahl der theoretisch minimal möglichen Kombinationen: $\underline{2}$

$2 \cdot 4 \cdot 4 \cdot 2 \cdot 0.2 \cdot 15 .\left(P \cup S / p o \ldots e / A_{2.2}\right) \cup(D-1)$

$$
\begin{aligned}
{[1 u \dot{c}, \dot{s}-i j](312)-[p o-1 G \dot{c}, \dot{s}-e-t,](-) } \\
\left.(P u S / p o \ldots e) ; M_{4} ; A_{2.2}\right) \cup(D-I) \\
{[k r a s, i v-i j](190)-} \\
{[p o-k r a s, i v,-e-t,](-) }
\end{aligned}
$$

Anzahl der tatsächlich realisierten Kombinationen: 2 
Anzahl der theoretisch maximal möqlichen Kombinationen: $\underline{2}$

Anzahl der theoretisch minimal möglichen Kombinationen: 1

- Theoretisch möglich ist ein Wegfall der Kombination $\left(P \cup S / p o \ldots e e^{;} A_{2.2}\right)$.

Somit verwirklicht das Russische zur Bildung von Verben mit der allgemeinen Explikation 'Etwas (jdn.) mit dem durch das ableitende Adjektiv benannte Merkmal versehen'

$$
\begin{array}{r}
12+3+7+2+4+1+4+4+3+2+4+5+2 \\
+2+2=57
\end{array}
$$

unterschiedliche Kombinationen der zur Verfügung stehenden Ausdrucksmittel.

Theoretisch maximal möglich sind

$$
\begin{array}{r}
29+8+20+4+14+2+12+5+4+4+18+12+3 \\
+8+2=145
\end{array}
$$

unterschiedliche Kombinationen.

Theoretisch minimal möglich sind

$$
\begin{aligned}
8+2+7+1+3 & +1+2+2+1+1+3+3+2 \\
+2+1 & =39
\end{aligned}
$$

unterschiedliche Kombinationen dieser Ausdrucksmittel.

2.4.4.2.1. Etwas (jdn.) in geringem Maße mit dem durch das ableitende Adjektiv benannte Merkmal vergehen

2.4.4.2.1.1. Aufzählung des Derivatemtyps sowie der jeweils miteinander kompatiblen Ausdrucksmittel zur Bildung von Verben mit dieser ollgemeinen Explikation

$2.4 .4 .2 .1 .1 .1 .0-I$

$$
\begin{aligned}
& \text { Pus/pod ... i / } \\
& M_{1.11} ; M_{4} \\
& A_{2.4}
\end{aligned}
$$

2.4.4.2.1.2. Die Kombinatorik dieser Mittel

$$
\begin{aligned}
& \left.2.4 .4 .2 .1 .2 .1 \text { (PuS/pod ... i /; M 1.11u4; } A_{2.4}\right) \cup(D-1) \\
& \text { [korótk,-ij] (91)-[pod-korot,-i-t,] (-) } \\
& \text { (PuS/pod ... i/; } \left.M_{4} ; A_{2.4}\right) \cup(D-I) \\
& {[\text { nov-ij] (1722) - [pod-nov, -i-t, ] (-) }}
\end{aligned}
$$


Zur Bildung von Verben mit der vorstehenden allgemeinen Explikation verwirklicht das Russische somit $\underline{2}$ unterschiedliche Kombinationen der zur Verfügung stehenden Ausdrucksmittel.

Theoretisch maximal möglich sind 4 unterschiedliche Kombinationen.

- Zusätzlich zu den realisierten sind die Kombinationen

(PuS/pod ... i/ ; $A_{2.4}$ ) sowie (PuS/pod ... i/; $M_{1.11} ; A_{2.4}$ ) theoretisch möglich.

Theoretisch minimal möglich ist 1 Kombination dieser Ausdrucksmittel.

- Theoretisch möglich ist ein Wegfall der Kombination ( $P \cup S / p o d \ldots i / ; M_{4} ; A_{2.4}$ ).

2.4.4.2.2. Etwas (jdn.) in stärkerem MaBe mit dem durch das ableitende Adjektiv benannte Merkmal versehen ${ }^{3}$

2.4.4.2.2.1. Aufzählung des Derivatemtyps sowie der jeweils miteinander kompatiblen Ausdrucksmittel zur Bildung von Verben mit dieser allgemeinen Explikation

$2 \cdot 4 \cdot 4 \cdot 2.2 \cdot 1.1 \cdot 0-1$

$$
\begin{aligned}
& \mathrm{PuS} / \text { po ... il } \\
& M_{1.11}, M_{1.13} ; M_{4} \\
& A_{1.2} \text { bei }[n, i z k,-i j](116) \text { - } \\
& {[v i s 6 k,-i j][(549) i z,-i-t,]} \\
& {[p o-v i s,-i-t,]} \\
& A_{2.4} \text { sonst }
\end{aligned}
$$

2.4.4.2.2.2. Die Kombinatorik dieser Mittel

$$
\begin{aligned}
& \left.2.4 .4 .2 .2 .2 .1 \text {. (PuS/po ... i ; } M_{1.11 \cup 4} ; A_{1.2}\right) \cup(D-I) \\
& {[n, i z k,-i j](116)-[p o-n, i z,-i-t,](B)} \\
& \text { (PuS/po ... i/; } M_{\left.1.13 \cup 4 ; A_{1.2}\right) \cup(D-I)} \\
& {[v i s o k,-i j](549)-[p o-v i s,-i-t,](40)}
\end{aligned}
$$

23 Ausprägungen dieser allgemeinen Explikation können in entsprechenden Kontexten auch viele derjenigen Verben realisieren, deren Bildung oben unter 2.4.4.2.0.2.10., 2.4.4.2.0.2.11., 2.4.4.2.0.2.12. sowie unter 2.4.4.2.0.2.15. beschrieben ist. 


$$
\begin{aligned}
& \left(P u S / p o \ldots i / ; M_{4} ; A_{2.4}\right) u(D-I) \\
& {[j a s n-i j](146)-[p o-j a s n,-i-t,]}
\end{aligned}
$$

Zur Bildung von Verben mit der vorstehenden allgemeinen Explikation verwirklicht das Russische somit 3 unterschiedliche Kombinationen der zur Verfügung stehenden Ausdrucksmittel.

Theoretisch maximal möqlich sind 10 unterschiedliche Kombinationen.

- Zusätzlich zu den realisierten sind die Kombinationen

$$
\begin{aligned}
& \left.\left(P \cup S / p o \ldots i / A_{1.2}\right) \text {, ( } P u S / p o \ldots i / A_{2.4}\right) \text {; } \\
& \left(P \cup S / p o \ldots \text { i/; } M_{1.11} ; A_{1.2}\right) \text {, (PuS/po ... il/; } M_{1.11} \text {; } \\
& \left.A_{2.4}\right) ;\left(P \cup S / p o \ldots \text { i } / M_{1.11 \cup 4} ; A_{2.4}\right) ;(P \cup S / p o \ldots i / ;
\end{aligned}
$$

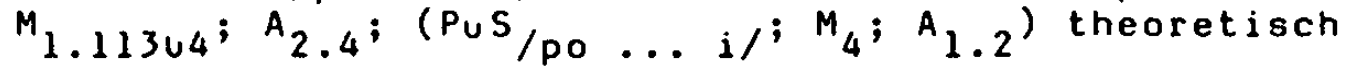

$$
\begin{aligned}
& \text { möglich. }
\end{aligned}
$$

Theoretisch minimal möqlich sind $\underline{3}$ unterschiedliche Kombinationen dieser Ausdrucksmittel.

2.4.4.2.3. Etwas (jdn.) übermäßig mit dem durch das ableitende Adjektiv benannte Merkmal versehen ${ }^{24}$

2.4.4.2.3.1. Aufzählung des Derivatemtyps sowie der jeweils miteinander kompatiblen Ausdrucksmittel zur Bildung von Verben mit dieser allgemeinen Explikation

$2 \cdot 4 \cdot 4 \cdot 2 \cdot 3 \cdot 1 \cdot 1 \cdot \quad D-1$

$$
\begin{aligned}
& \text { Pus/iz ... i/ v/is ... il } \\
& M_{1.11} \text { bei }[r e ́ d k,-i j]\left(\begin{array}{l}
(80) \\
{[i z-r, e d,-i-t,](-)}
\end{array}\right. \\
& M_{4} ; M_{6.1}
\end{aligned}
$$

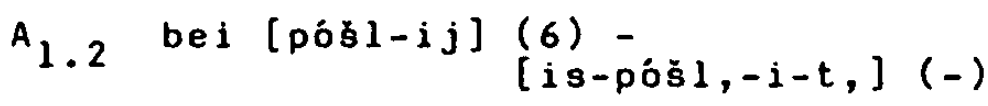

$$
\begin{aligned}
& A_{2.4} \text { sonst } \\
& 2.4 \cdot 4 \cdot 2 \cdot 3 \cdot 1.2 \text {. D-I } \\
& \text { PuS/p,er,e ... il }
\end{aligned}
$$

24 Ausprägungen dieser allgemeinen Explikation können in entsprechenden Kontexten auch einige von den Verben realisieren, deren Bildung unte: 2.4.4.2.0.2.5., 2.4.4.2.0.7. sowie unter 2.4.4.2.0.2.8. beschrieben ist. 


$$
\begin{aligned}
& M_{4} ; M_{5} ; M_{6.1} \\
& A_{2.4}
\end{aligned}
$$

2.4.4.2.3.2. Die Kombinatorik dieser Mittel

$$
\begin{aligned}
& 2.4 .4 .2 .3 .2 .1 . \quad\left(P \cup S / i z \ldots . i / M_{1.11 \cup 4} ; A_{2.4}\right) \cup(D-I) \\
& {[r, \dot{e} d \hat{k},-i j](80 j-[i z-r, e d,-i-t,](-)} \\
& \left(P u S / \text { is ... i/ ; } M_{4} ; A_{1.2}\right) \cup(D-I) \\
& \text { [póšl-ij] (6) - [is-póśl, -i-t, ] (-) } \\
& \text { (Pus/is ... i/; } M_{6.1} ; A_{2.4} \text { ) u(D-I) } \\
& \text { [tónk, -ij] }(162)-[i s-t o n c,-i-t,](-)
\end{aligned}
$$

Anzahl der tatsächlich realisierten Kombinationen: 3

Anzahl der theoretisch maximal möglichen Kombinationen: 10

- Zusätzlich zu den realisierten sind die Kombinationen

$$
\begin{aligned}
& \left(P \cup S / i z \ldots i / v / i s \ldots i / A_{1.2}\right) \text {, } \\
& \left(P \cup S / i z \ldots i / v / i s \ldots i / A_{2.4}\right) \text {; } \\
& \left(P \cup S / i z \ldots i / v / \text { is } \ldots \text { i/; } M_{1.11} ; A_{1.2}\right) \text {, } \\
& \left(P \cup S / i z \ldots \text { i/ v/is ... i/; } M_{1.11} ; A_{2.4}\right. \text { ); } \\
& \text { (Pus/iz ... i/ v/is ... i/; M } \left.1.11 \cup 4 ; A_{1.2}\right) \text {; } \\
& \left(P \cup S / i z \ldots \text { i/ } \vee / i s \ldots i / M_{4} ; A_{2.4}\right) \text {; } \\
& \text { (Pus/iz ... i/ v/is ... i/; } M_{6.1} ; A_{1.2} \text { ) theoretisch mög- } \\
& \text { lich. }
\end{aligned}
$$

Anzahl der theoretisch minimal möglichen Kombinationen: $\underline{3}$

$\left.2.4 .4 .2 .3 .2 .2 . \quad(P \cup S / p, e r, e, \ldots i) ; M_{4} ; A_{2.4}\right) u(D-I)$

$$
\begin{aligned}
& {[k, i s l-i j](13)-[p, e r, e-k, i s l,-i-t,](-)} \\
& \left(P \cup S / p, e r, e \ldots \text { il); } M_{S \cup 6.1} ; A_{2.4}\right) \cup(D-I)
\end{aligned}
$$$$
[g o r, k,-i j](43)-[p, e r, e-g o r c,-i-t,](-)
$$$$
\text { (Pus/p,er,e ...i i ; } \left.M_{6.1} ; A_{2.4}\right) \cup(D-1)
$$

[tónk,-ij] $(162)-[p, e r, e-t o n c,-i-t],(-)$

Anzahl der tatsächlich realisierten Kombinationen: 3

Anzahl der theoretisch maximal möqlichen Kombinationen: 4

- Zusätzlich zu den realisierten ist die Kombination (PuS/p,er,e ... i/; $A_{2.4}$ ) theoretisch möglich.

Anzahl der theoretisch minimal möglichen Kombinationen: $\underline{2}$

- Theoretisch möglich ist ein Wegfall der Kombination 


$$
\left(P_{u} / p, \text { er,e } \ldots \text { i/; } M_{6.1} ; A_{2.4}\right) \text {. }
$$

Somit verwirklicht das Russische zur Bildung von Verben mit der allgemeinen Explikation 'Etwas (jdn.) übermäBig mit den durch das ableitende Adjektiv benannte Merkmal versehen'

$$
3+3=6
$$

unterschiedliche Kombinationen der zur Verfügung stehenden Ausdrucksmittel.

Theoretisch maximal möglich sind

$$
10+4=14
$$

unterschiedliche Kombinationen dieser Mittel.

Theoretisch minimal möglich sind

$$
3+2=5
$$

unterschiedliche Kombinationen.

2.4.4.3. Etwas (jdn.) des mit dem ableitenden Adjektiv benannten Merkmals berauben

2.4.4.3.1. Aufzählung der Derivatemtypen sowie der jeweils miteinander kompatiblen Ausdrucksmittel zur Bildung von Verben mit dieser allgemeinen Explikation

$2 \cdot 4 \cdot 4 \cdot 3 \cdot 1.1 . D-1, D-11 / 1$

$$
\begin{aligned}
& \mathrm{PuS}_{\mathrm{raz}} \ldots \mathrm{i} / \mathrm{f} / \mathrm{ras} \ldots \mathrm{i} / 2 \\
& \mathrm{M}_{4} \\
& \mathrm{~A}_{2.2}, \mathrm{~A}_{2.4}
\end{aligned}
$$

2.4.4.3.2. Die Kombinatorik dieser Mittel

$$
\begin{aligned}
& 2.4 .4 .3 .2 .1 . \quad\left(P u S / r a z \ldots i / 2 ; A_{2.4}\right) \cup(D-1) \\
& {[o ́ b s ̌ c ́,-i j](401)-[\operatorname{raz}-o b \dot{s} \dot{c},-i-t,](-)} \\
& \left(P \cup S / \text { ras ... i/ } ; M_{4} ; A_{2.2}\right) \cup(D-I I / 1) \\
& {[s, e k r, e ́ t n-i j](10)-[r a s-s, e k r, e ́ t,-i-t,](-)} \\
& \text { (Pus/ras ...i i/2; } \left.M_{4} ; A_{2.4}\right) \cup(D-I) \\
& [k, i s]-i j](13)-[\operatorname{ras}-k, i s],-i-t,](-)
\end{aligned}
$$

Zur Bildung von Verben mit der vorstehenden allgemeinen Explikation verwirklicht das Russische somit $\underline{3}$ unterschiedliche 
Kombinationen der zur Verfügung stehenden Ausdrucksmittel.

Theoretisch maximal möglich sind $\underline{4}$ unterschiedliche Kombinationen.

- Zusätzlich zu den realisierten ist die Kombination (Pus/raz ...i i/ v/ras ...i/ $; A_{2.2}$ ) theoretisch mög1 ich.

Theoretisch minimal möglich sind $\underline{2}$ unterschiedliche Kombinationen dieser Ausdrucksmittel.

- Theoretisch möglich ist ein Wegfall der Kombination (PuS/raz ... i/ v/ras ... i/ $; \mathrm{M}_{4} ; \mathrm{A}_{2.4}$ ).

Im Rahmen der deadjektivischen Derivation von Verben ${ }^{25}$ nutzt das Russische, wie gezeigt, insgesamt

$$
36+57+2+3+6+3=107
$$

unterschiedliche Kombinationen der im analytischen Teil dieser Arbeit je für sich beschriebenen Ausdrucksmittel aus.

Theoretisch maximal möglich sind

$$
69+145+4+10+14+4=246
$$

unterschiedliche Kombinationen.

Theoretisch minimal möglich sind

$$
26+39+1+3+5+2=76
$$

unterschiedliche Kombinationen dieser Ausdrucksmittel.

25 Vgl. zu den Zahlenangaben die Seiten 146, 158, 159, 160, 162 (sowie 163). 
2.4.5. Deadjektivische Adverbien

2.4.5.1. Hervortreten des mit dem ableitenden Adjektiv benannten Merkmals in verstärktem MaBe

2.4.5.1.1. Aufzählung der Derivatemtypen sowie der jeweils miteinander kompatiblen Ausdrucksmittel zur Bildung von Adverbien mit dieser allgemeinen Explikation

2.4 .5 .1 .1 .1$. D-I, D-II/I, D-III/7

S/im/

$\mathrm{M}_{5}$

$A_{1.3}$

$2.4 .5 .1 .1 .2 . \quad D-1$

PuS/na $\ldots o / 1$

$\mathrm{M}_{5}$

A.1

2.4.5.1.2. Die Kombinatorik dieser Mittel

$$
\begin{aligned}
& 2.4 .5 .1 .2 .1 . \quad\left(S / \mathrm{im} / ; A_{1.3}\right) \cup(D-I I / 1) \\
& [s v, e ́ t]-i j] \underset{[s v, e t 1-i m(-s v, e t l o)](-)}{(116)-} \\
& \left(S / \mathrm{im} / ; A_{1.3}\right) \cup(D-I I I / 7) \\
& {[p, a ́ n-i j](88)-[p, a n-i m(-p \text {,anó })](-)} \\
& \left(\mathrm{S} / \mathrm{im} / \mathrm{M}_{5} ; A_{1.3}\right) \cup(D-1) \\
& \text { [dávn-ij] (35) - [davn,-im (-davnó)] }
\end{aligned}
$$

Anzahl der tatsächlich realisierten Kombinationen: $\underline{2}$

Anzahl der theoretisch maximal möglichen Kombinationen:

Anzahl der theoretisch minimal möqlichen Kombinationen: :

- Theoretisch möglich ist ein Wegfall der Kombination $\left(S_{/ i m /} ; A_{1.3}\right)$.

2.4.5.1.2.2. (PuS/na ... o/,; $\left.A_{1.1}\right) \cup(D-I)$

[pérv-ij] (1605) -

$[(p, \dot{e} r v o-) n a ́-p, e r v-o](-)$ 


$$
\begin{aligned}
& \left(P \cup S / n a \ldots o /, ; M_{5} ; A_{1.1}\right) \cup(D-I) \\
& \quad[k r, \text { épk, }-i j](70)- \\
& {[(k r, \text { épko- ) ná-kr,epk-o }]}
\end{aligned}
$$

Anzahl der tatsächlich realisierten Kombinationen: $\underline{2}$

Anzahl der theoretisch maximal möglichen Kombinationen: $\underline{2}$

Anzahl der theoretisch minimal möglichen Kombinationen: $\underline{1}$

- Theoretisch möglich ist ein Wegfall der Kombination (PUS/na ... o/ $; A_{1.1}$ ).

Somit verwirklicht das Russische zur Bildung von Adverbien mit der allgemeinen Explikation 'Hervortreten des mit dem ableitenden Adjektiv benannten Merkmals in verstärktem MaBe'

$$
2+2=4
$$

unterschiedliche Kombinationen der zur Verfügung stehenden Ausdrucksmittel.

Theoretisch maximal möglich sind gleichfalls

$$
2+2=4
$$

unterschiedliche Kombinationen.

Theoretisch minimal möglich sind

$$
1+1=2
$$

unterschiedliche Kombinationen dieser Ausdrucksmittel.

2.4.5.2. Uberführung von etwas (jdn.) in einen Zustand, der sich durch das mit dem ableitenden Adjektiv benannte Merkmal auszeichnet

2.4.5.2.1. Aufzählung der Derivatemtypen sowie der jeweils miteinander kompatiblen Ausdrucksmittel zur Bildung von Adverbien mit dieser allgemeinen Explikation

$2.4 .5 .2 .1 .1 . \quad D-1, D-111 / 7$

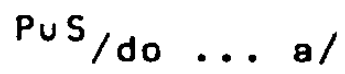

$$
\begin{aligned}
& \begin{array}{r}
A_{1,1} \text { be } i \quad[s, i n,-i j](180)-[d o-s, i n,-a] \\
{[s i t-i j](25)-[d o-s i t-a](7)}
\end{array} \\
& {[\text { sux-ój] }(87)-[d o-s u x-a](-)} \\
& A_{1.3}
\end{aligned}
$$

$2 \cdot 4 \cdot 5 \cdot 2 \cdot 1.2 \cdot \quad D-1$

$$
\text { Pus/na ... o/ } 2
$$


$\mathrm{M}_{5}$

$A_{1.1}$

$A_{1.2}$ bei $[v, e ́ c ́, n-i j](90)-$

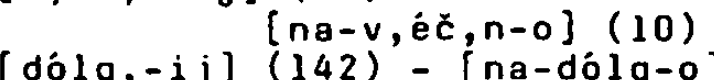

\subsection{Die Kombinatorik dieser Mittel}

$2.4 .5 .2 .2 .1 . \quad\left(P U S / d o \ldots\right.$ a $\left./ A_{1.1}\right) \cup(D-I)$

$$
[s, i n,-i j](180)-[d o ́-s, i n,-8]
$$

(PuS/do ... a/; $\left.A_{1.3}\right) \cup(D-1)$

[gól-ij] (73)-[do-gol-á] (1)

$\left(P \cup S / d o \ldots a / ; A_{1.3}\right) \cup(D-I I I / 7)$

$[p, a ́ n-i j](88)-[d o-p, a n-a ́ a](-)$

Anzahl der tatsächlich realisierten Kombinationen: $\underline{2}$

Anzahl der theoretisch maximal möglichen Kombinationen: $\underline{2}$

Anzahl der theoretisch minimal möglichen Kombinationen: $\underline{2}$

$2.4 .5 .2 .2 .2 . \quad\left(P u S / n a \ldots o / 2 ; A_{1.1}\right) u(D-I)$

$[g] u x-o j](54)-[n a ́-g l u x-0](10)$

$\left(P \cup S / n a \ldots o / 2 ; A_{1.2}\right) \cup(D-I)$

$[v, e ́ c \dot{c}, n-i j](90)-[n a-v, e ́ c ́, n-0]$

(PuS/na ... o/ ; $\left.M_{5} ; A_{1.1}\right) \cup(D-I)$

$[k r, e ́ p k,-i j](70)-[n a ́-k r, e p k-0]$

(PuS/na $\left.\ldots o / 2 ; M_{5} ; A_{1.2}\right) \cup(D-I)$

[dólg,-ij] (142)-[na-dólg-o](24)

Anzahl der tatsächlich realisierten Kombinationen: 4

Anzahl der theoretisch maximal möqlichen Kombinationen: 4

Anzahl der theoretisch minimal möglichen Kombinationen: 2

- Theoretisch möglich ist ein Wegfall der Kombinationen $\left(P u S / n a \ldots o / 2 ; A_{1.1}\right)$ und $\left(P u S / n a \ldots o / 2 ; M_{5} ; A_{1.2}\right)$.

Zur Bildung von Adverbien mit der allgemeinen Explikation - Uberführung von etwas (jdn.) in einen Zustand, der sich durch das mit dem ableitenden Adjektiv benannte Merkmal auszeichnet. 
verwirklicht das Russische somit

$$
2+4=6
$$

unterschiedliche Kombinationen der zur Verfügung stehenden Ausdrucksmittel.

Maximal möglich sind theoretisch ebenfalls

$$
2+4=6
$$

unterschiedliche Kombinationen.

Theoretisch minimal möglich sind

$$
2+2=4
$$

unterschiedliche Kombinationen dieser Ausdrucksmittel.

2.4.5.3. Gerichtetheit auf das mit dem ableitenden Adjektiv benannte Merkmal

2.4.5.3.1. Aufzählung des Derivatemtyps sowie der jeweils miteinander kompatiblen Ausdrucksmittel zur Bildung von Adverbien mit dieser allgemeinen Explikation

$2 \cdot 4 \cdot 5 \cdot 3 \cdot 1.1 .0-1$

$$
\begin{aligned}
& P_{u S} / \mathrm{na} \ldots \mathrm{o} / 3 \\
& \text { A }_{1.2}
\end{aligned}
$$

$2 \cdot 4 \cdot 5 \cdot 3 \cdot 1.2$. D-I

$$
\begin{aligned}
& \text { PuS /v ... ol } \\
& \text { A }_{1.2}
\end{aligned}
$$

2.4.5.3.2. Die Kombinatorik dieser Mittel

$$
\begin{aligned}
2.4 .5 .3 .2 .1 . \quad & \left(P_{u} / \mathrm{na} \ldots o / 3 ; A_{1.2}\right) u(D-1) \\
& {[p r a ́ v-i j](204)-[n a-p r a ́ v-0](41) } \\
& \left(P u S / n a \ldots o / 3 ; A_{1.2}\right) u(D-1) \\
& {[1, \dot{e} v-i j](117)-[n a-1, \dot{e} v-0](31) }
\end{aligned}
$$

Anzahl der tatsächlich realisierten Kombinationen: 1

Anzahl der theoretisch maximal möglichen Kombinationen: $\underline{1}$

Anzahl der theoretisch minimal möglichen Kombinationen: 1 
$2.4 .5 .3 .2 .2 . \quad\left(P u S / v \ldots \circ ; A_{1.2}\right) u(D-I)$

$$
\begin{aligned}
& {\left[\text { práv-ij] }(204)^{2}-[v-p r a ́ v-0]\right. \text { (4) }} \\
& \left(P_{u} S / v \ldots \text { of } A_{1,2}\right) u(D-1) \\
& {[1, \dot{e} v-i j](117)^{2}-[v-1, \dot{e} v-0] \text { (8) }}
\end{aligned}
$$

Anzahl der tatsächlich realisierten Kombinationen: 1

Anzahl der theoretisch maximal möqlichen Kombinationen: 1

Anzahl der theoretisch minimal möglichen Kombinationen: 1

Somit verwirklicht das Russische zur Bildung von Adverbien mit der allgemeinen Explikation 'Gerichtetheit auf das mit dem ableitenden Adjektiv benannte Merkmal'

$$
1+1=2
$$

unterschiedliche Kombinationen der zur Verfügung stehenden Ausdrucksmittel.

Theoretisch maximal wie auch minimal möglich sind gleichfalls

$$
1+1=2
$$

unterschiedliche Kombinationen.

2.4.5.4. Seinen Anfang bei dem nohmen bzw. von dem ausgehen, was mit dem ableitenden Adjektiv benannt ist

2.4.5.4.1. Aufzählung der Derivatemtypen sowie der jeweils miteinander kompatiblen Ausdrucksmittel zur Bildung von Adverbien mit dieser allgemeinen Explikation

$2 \cdot 4 \cdot 5 \cdot 4 \cdot 1.1 . \quad D-1$

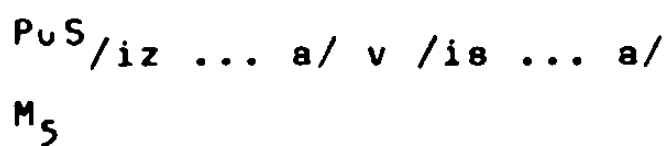

$A_{1.1}$

$A_{1.2}$ bei $[\grave{c}, u \dot{z}-b j](171)-[i s-\grave{c}, \dot{u} \dot{z}-a](-)$

$A_{1.3}$ bei $[d a l, o ́ k,-i j](107)$ -

[iz-dal,ok-á] (32)

$2.4 .5 .4 .1 .2 . D-1, D-I I 1 / 1, D-111 / 7$

Pus/s .... a/

$\mathrm{M}_{3.6} ; \mathrm{M}_{5}$

$A_{1.2}$ 


$$
\begin{aligned}
& \begin{aligned}
A_{1.3} \text { bei } & {[v i s o ́ k,-i j](549)-[s-v i s o k-a ́] } \\
& {[g \circ r, a c ́,-i j](163)-}
\end{aligned} \\
& {[p, a ́ n-i j](88)[8-g \circ r, a c ́,-a ́](9)}
\end{aligned}
$$

2.4.5.4.1.3. D-I, D-III/7

$$
\begin{aligned}
& \mathrm{PuS}_{/ \mathrm{s}} \ldots \mathrm{u} / \mathrm{v} / \mathrm{so} \ldots \mathrm{u} / \\
& { }_{1.12} \text { bei [durn-ój] (48) - [8-dúr-u] } \\
& { }_{1.1}
\end{aligned}
$$

$2.4 \cdot 5 \cdot 4 \cdot 1.4 . \quad D-1$

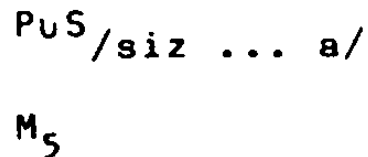

$A_{1.1}$

2.4.5.4.2. Die Kombinatorik dieser Mittel

$$
\begin{aligned}
& 2.4 .5 .4 .2 .1 . \quad\left(P \cup S / \text { is } \ldots \text { a/; } A_{1.2}\right) \cup(D-I) \\
& {[\grave{c}, u \grave{z}-\dot{o} j](171)-[i s-\grave{c}, u ́ \dot{z}-a](-)} \\
& \left(P \cup S / i z \ldots \text { a/; } M_{5} ; A_{1.1}\right) \cup(D-I) \\
& \text { [dávn,-ij] (35)-[iz-davn-a] (9) } \\
& \text { (PuS/iz ... a/; } \left.M_{5} ; A_{1.3}\right) \cup(D-I) \\
& \text { [dal,ók, -ij] (107)-[iz-dal,ok-á] }
\end{aligned}
$$

Anzahl der tatsächlich realisierten Kombinationen: 3

Anzahl der theoretisch maximal möglichen Kombinationen: 6

- Zusätzlich zu den realisierten sind die Kombinationen

$\left(\right.$ PuS/iz ... a/ v/is ... a/; $\left.A_{1.1}\right)$,

$\left(P \cup S / i z \ldots a / v /\right.$ is $\ldots$ a/ A $\left._{1.3}\right)$;

$\left(\right.$ PuS $/ i z \ldots a / v /$ is ... $\left.8 / ; M_{5} ; A_{1.2}\right)$ theoretisch möglich.

Anzahl der theoretisch minimal möglichen Kombinationen: 3

$2.4 .5 .4 .2 .2 . \quad\left(P \cup S / s \ldots a^{\prime} ; A_{1.2}\right) \cup(D-1)$

$$
\text { [glúp-ij] (99)-[s-glúp-8](-) }
$$

$\left(P \cup S / s \ldots a / ; A_{1.3}\right) \cup(D-I I I / 1)$

[gor,áć,-ij] (163)-[s-gor, ać,-á] (9)

$\left(P \cup S / s \ldots\right.$ a/; $\left.A_{1.3}\right) \cup(D-111 / 7)$

$[p, a ́ n-i j](88)-[s-p, a n-\dot{a}](-)$ 


$$
\begin{aligned}
& \left(\mathrm{PuS} / \mathrm{s} \ldots \text { al; } \mathrm{M}_{3.6} ; \mathrm{A}_{1.2}\right) \cup(\mathrm{D}-\mathrm{I}) \\
& \text { [molod-ój] }(608)-[s-m l a ́ d-a](-) \\
& \text { ( Pus } / \mathrm{s} \ldots \text {. a/; } \mathrm{M}_{5} ; \mathrm{A}_{1.3} \text { ) } \cup(\mathrm{D}-\mathrm{I}) \\
& \text { [visók,-ij] (549)-[s-visok-á] }
\end{aligned}
$$

Anzahl der tatsächlich realisierten Kombinationen: 4

Anzahl der theoretisch maximal möqlichen Kombinationen: 6

- Zusätzlich zu den realisierten sind die Kombinationen $\left(P \cup S / s \ldots\right.$ a $\left.; M_{3.6} ; A_{1.3}\right) ;\left(P u S / s \ldots\right.$. $\left./ M_{5} ; A_{1.2}\right)$ theoretisch möglich.

Anzahl der theoretisch minimal möglichen Kombinationen: $\underline{2}$

- Theoretisch möglich ist ein Wegfall der Kombinationen $\left(\mathrm{PuS} / \mathrm{s} \ldots \mathrm{a} / \mathrm{A}_{1.2}\right)$ und $\left(\mathrm{PuS} / \mathrm{s} \ldots \mathrm{a} / \mathrm{A}_{1.3}\right)$.

$2.4 .5 .4 .2 .3 . \quad\left(P \cup S / s \ldots u / A_{1.1}\right) u(D-I)$

[molod-ój] $(608)-[s-\operatorname{mólod}-u](3)$

$$
\begin{aligned}
& \left(P \cup S / 80 \ldots u / A_{1.1}\right) \cup(D-I) \\
& {[s 1, e p-o j](31)-[s o ́-s l, e p-u]} \\
& \left(P \cup S / 8 \ldots u / ; A_{1.1}\right) \cup(D-I I I / 7) \\
& {[p, a ́ n-i j](88)-[s-p, a ́ n-u](2)} \\
& \text { (PuS/s ...u } \left./ M_{1.12} ; A_{1.1}\right) \cup(D-I) \\
& \text { [durn-ój] (48)-[8-dúr-u] (1) }
\end{aligned}
$$

Anzohl der tatsächlich realisierten Kombinationen: 2 Anzohl der theoretisch maximal möglichen Kombinationen: $\underline{2}$ Anzahl der theoretisch minimal möglichen Kombinationen: 1

- Theoretisch möglich ist ein Wegfall der Kombination $\left(P_{u S} / \mathrm{s} \ldots u / v /\right.$ so ... u/ ; $\left.A_{1.1}\right)$.

$$
\begin{aligned}
& 2.4 .5 .4 .2 .4 . \quad\left(P \cup S / 8 i z \ldots \text { a/; } A_{1.1}\right) u(D-I) \\
& \text { [nóv-ij] (1722)-[siz-nov-8] (4) } \\
& \text { (PuS/siz ... a/; } M_{5} ; A_{1.1} \text { ) u(D-I) } \\
& \text { [dávn,-ij] (35)-[8iz-davn-a] (-) }
\end{aligned}
$$

Anzahl der tatsächlich realisierten Kombinationen: $\underline{2}$ Anzahl der theoretisch maximal möglichen Kombinationen: $\underline{2}$ Anzahl der theoretisch minimal möglichen Kombinationen: 1

- Theoretisch möglich ist ein Wegfall der Kombination (Pus/siz ... a/; $A_{1.1}$ ). 
Zur Bildung von Adverbien mit der allgemeinen Explikation 'Seinen Anfang bei dem nehmen bzw. von dem ausgehen, was mit dem ableitenden Adjektiv benant ist' verwirklicht das Russische somit

$$
3+4+2+2=11
$$

unterschiedliche Kombinationen der zur Verfügung stehenden Ausdrucksmittel.

Theoretisch maximal möglich sind

$$
6+6+2+2=16
$$

unterschiedliche Kombinationen.

Theoretisch minimal möglich sind

$$
3+2+1+1=7
$$

unterschiedliche Kombinationen dieser Ausdrucksmittel.

Im Rahmen der deadjektivischen Derivation von Adverbien ${ }^{26}$ nutzt das Russische, wie gezeigt, insgesamt

$$
4+6+2+11=23
$$

unterschiedliche Kombinationen der im analytischen Teil dieser Arbeit je für sich beschriebenen Ausdrucksmittel aus.

Theoretisch maximal möglich sind

$$
4+6+2+16=28
$$

unterschiedliche Kombinationen.

Theoretisch minimal möglich sind

$$
2+4+2+7=15
$$

unterschiedliche Kombinationen dieser Ausdrucksmittel.

Insgesamt ${ }^{27}$ realisiert das Russische im Rahmen der deadjektivischen Derivation von Substantiven, Adjektiven, Verben und Adverbien

$$
109+39+107+23=278
$$

26 Zu den Zahlenangaben vgl. die Seiten 165, 167, 168 (und 171).

$27 \mathrm{Vgl}$. zu diesen Zahlenangaben die Seiten 124, 138, 163 (sowie 171). 
unterschiedliche Kombinationen von Ausdrucksmitteln.

Theoretisch maximal möglich wären

$$
236+56+246+28=566
$$

unterschiedliche Kombinationen.

Minimal möglich wären theoretisch

$$
81+34+76+15=206
$$

unterschiedliche Kombinationen der im analytischen Teil dieser Arbeit je für sich aufgeführten Ausdrucksmittel. 
2.4.6. Die Bestimmung des 'MaBes der Verbundenheit' für die deadjektivische Derivation des Russischen

Die oben (2.4.1. - 2.4.5.) ermittelten Werte gestaten eine Berechnung dessen, welchen Grad der Verbundenheit die deadjektivische Derivation des Russischen hinsichtlich der Beziehungen zwischen den einzelnen Ausdrucksmitteln aufweist, mit onderen Worten, inwieweit hier implikative Beziehungen vorliegen.

Maximale Unverbundenheit wäre dann gegeben, wenn sich jedes Ausdrucksmittel einer Klasse (z.B. der Klasse P, PuS, $S$, SUPo) mit jedem Mittel der jeweils anderen Klassen (also der Klasse $\underline{M}$ und der Klasse $\underline{A}$ ) verbinden könnte. Im hier interessierenden Bereich enthält die Klasse der Affixe 90 unterschiedliche Ausdrucksmittel, die Klasse der morphonologischen Alternationen 69, und hinsichtlich des Akzents haben wir 13 unterschiedliche Konfigurationen festgestellt. Bestünden bei der deadjektivischen Derivation keinerlei implikative Beziehungen zwischen den einzelnen Ausdrucksmitteln der jeweiligen Klassen, so läge ma$x$ imale Unverbundenheit vor, und wir hätten es mit

$$
90 \times 69 \times 13=80730
$$

unterschiedlichen Kombinationen zu tun. Der höchste Grad der Verbundenheit, mithin die kleinstmögliche Zahl von Kombinationen, bestimmt sich durch die Anzahl der Elemente in der Klasse mit den meisten Ausdrucksmitteln, in unserem Fall durch die Anzahl der Mittel in der Klasse der Affixe (90).

Aufgrund der in der Einleitung zum synthetischen Teil dieser Arbeit getroffenen Entscheidungen (vgl. $98 \mathrm{ff}$ ) reduziert sich die Zahl der maximal möglichen Kombinationen ( $K_{\max }$ ) ganz erheblich auf 566, wohingegen die Zahl der minimal möglichen Kombinationen ( $K_{m i n}$ ) vergleichsweise geringfügig auf 206 ansteigt. Ferner beträgt die Anzahl der tatsächlich realisierten Kombinationen ( $K_{\text {real }}$ ), wie gezeigt, 278 , so dab nun der Wert des Masses der Verbundenheit' ermittelt werden kann, das wie folgt definiert ist:

$$
M(V)_{L}=\frac{K_{\max }-K_{\text {real }}}{K_{\max }-k_{\min }}
$$

Da die Werte dieses MaBes im Intervall<0;l> liegen, lossen sich 
vermittels seiner verschiedene 5prachen untereinander vergleichen. Wenn wir nun die oben (17lf) ermittelten Gesamtwerte für die deadjektivische Derivation des Russischen einsetzen, so erhalten wir

$$
M(V)_{\text {Russ (deadDges) }}=\frac{566-278}{566-206}=0.8^{28}
$$

Genauso wie der Wert des Gesamtmaßes ermittelt wurde, lassen sich aufgrund der vorangegangenen Deskription auch die Untermaße bis hin zu den Kombinationen derjenigen Mittel bestimmen, die zum Ausdruck der einzelnen allgemeinen Explikationen beitragen. Hier mag es jedoch genügen, die entsprechenden Werte für die deadjektivische Derivation von Substantiven (deadDS), von Adjektiven (deadDAdj), von Verben (deadDV) und von Adverbien (deadDAdv) nach der obigen allgemeinen formel zu berechnen:

$$
\begin{aligned}
& M(V)_{\text {Russ (deadDS) }}=\frac{236-109}{236-81}=0.819 \\
& M(V)_{\text {Russ (deadDAdj) }}=\frac{56-39}{56-34}=0.773 \\
& M(V)_{\text {Russ (deadDV) }}=\frac{246-107}{246-76}=0.818 \\
& M(V)_{\text {Russ (deadDAdv) }}=\frac{28-23}{28-15}=0.385
\end{aligned}
$$

Beim Vergleich der Resultate fält auf, daß die Werte für $M(V)$ (deadDS), $M(V)$ (deadDAdj) sowie $M(V)$ (deadDV) nur mehr oder weniger unwesentlich vom Wert des Gesamtmabes differieren, während $z$ wischen den Ausdrucksmitteln im Bereich der deadjektivischen Derivation von Adverbien deutlich weniger implikative Beziehungen bestehen.

Diese Beziehungen in Form von Regelhaftigkeiten kenntlich zumachen, wird die Aufgabe des nächsten Kapitels der vorliegenden Arbeit sein.

28 Dieser Wert liegt um 0.05 höher als dies etwa bei der Präsensformenbildung des Russischen der Fall ist (vgl. LEHFELDT 1978b, 45). Ein noch höherer Wert ließe sich bei der Verwendung von $k_{\max }=80730, k_{\min }=90$ und $k_{\text {real }}=278$ erzielen, nämlich 0.998 . 
2.4.7. Die implikativen Regelhaftigkeiten bei der Kombination der Ausdrucksmittel

Die im vorangegangenen Kapitel erfolgte Bestimmung des 'MaBes der Verbundenheit ' für die deadjektivische Derivation hat gezeigt, dab das Russiche in diesem Bereich bezüglich der Kombinationen der Ausdrucksmittel insgesamt einen recht hohen Grad an implikativen Regelhaftigkeiten aufweist, die nun in graphischer form abgebildet werden sollen.

Jeder der unten aufgeführten Graphen zeigt die Beziehungen zwischen den Mitteln auf, die an Ausdruck der jeweiligen allgemeinen Explikationen beteiligt sind und ist folglich je einer solchen zugeordnet. Hinsichtlich der Art dieser Beziehungen werden wir fünf Typen implikativer Regelhaftigkeiten unterscheiden. Dies sei an einem Beispiel erläutert.

Zur Ableitung von deadjektivischen Substantiven mit der allgemeinen Explikation 'Jemand (etwas), der (das) sich durch ein bestimmtes Merkmal auszeichnet', sind neben anderen die folgenden Ausdrucksmittel untereinander kombiniert (vgl.o., 121):

0.12 .3

Dieser Graph ist folgendermaBen zu lesen:

Wenn $M_{\text {g oder }} M_{1.12}$

wenn $M_{1} .12$ vorkommt, dann kommt auch $A_{2.2}$ vor;

wenn $M_{2.244}$ oder $M_{2.10 u 4}$ oder $M_{2.19}$ oder $M_{1.12 \cup 2.19}$ vorkommen, dann kommt auch $A_{1.2}$ vor. 
Dieser Typ einer prädiktiven Regel ist im Graphen so notiert:

(1) (wenn $x$, dann eindeutig $y$ ): $x \longrightarrow y$

Neben diesen eindeutigen lassen sich auch eineindeutige Beziehungen nachweisen:

Wenn $M_{3.1}$ vorkommt, dann kommt auch $A_{2.1}$ vor und umgekehrt; wenn $M_{6.1}$ vorkommt, dann kommt auch $A_{2.3}$ vor und umgekehrt. Dieser zweite Typ ist allgemein folgendermaßen notiert:

(2) (wenn $x$, dann eineindeutig $y$ ): $x \longleftrightarrow y$

Entweder-oder-Beziehungen (in unserem Graphen sind dies: (a) wenn $S / k_{2} /$, dann entweder $M_{\emptyset}$ oder $M_{1.12}$ oder $M_{2.2}$ oder $M_{2.10 u 4}$ oder $M_{2.19}$ oder $M_{3.1}$ oder $M_{6.1}$; (b) wenn $M_{\emptyset}$, dann entweder $A_{2.2}$ oder $A_{1.2}$; (c) wenn $A_{2.2}$, dann entweder $M_{g}$ oder $M_{1.12}$; (d) wenn $A_{1.2}$, dann entweder $M_{g}$ oder $M_{2.204}$ oder $M_{2.1044}$ oder $M_{2.19}$ oder $M_{1.12 \cup 2.19}$ ) werden nicht mit gerichteten, sondern mit ungerichteten Kanten gekennzeichnet. Allgemein:

(3) (wenn $x$, dann entweder $y$ oder $z$ ):

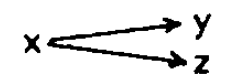

Beziehungen nach der Art der drei bisher unterschiedenen Typen haben lediglich innerhalb desjenigen Graphen Gültigkeit, in dem sie vorkommen. So fordert z.B. M 1.12 zwar innerhalb des obigen Graphen $\mathrm{S} / \mathrm{k}_{2} /$, in anderen Graphen jedoch andere Ausdrucksmittel aus der Klasse $P$, PuS, S, SUPo; $M$ gteht hier in einer entwederoder-Beziehung zu Ausdrucksmitteln der Klasse $\underline{A}$, während in anderen Graphen diese Beziehung eindeutig oder eineindeutig ist.

Die beiden folgenden Typen implikativer Regelhaftigkeiten gelten dagegen für den gesamten Bereich der deadjektivischen Wortbildung und weisen so über den einzelnen Graphen hinaus:

$M_{2.204}, M_{2.1044}, M_{2.19}$ sowie $M_{1.1202 .19}$ prädizieren eindeutig $\mathrm{S} / \mathrm{k}_{2} /$. Mit anderen Worten: Die genannten morphonologischen Alternationen sind $m i t$ anderen Affixen als zusammen mit $5 / k_{2} /$ nicht belegt. Allgemein:

(4) (x prädiziert eindeutig $y$ ): $x \longrightarrow y$ 
Beziehungen der Art

(5) ( $x$ prädiziert in Verbindung mit $v$ eindeutig $y$ ):

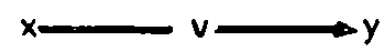

können dann unmittelbar aus dem Graphen abgelesen werden, wenn ' $x$ ' oder ' $v$ ' der Klasse $P, P u S, S$, SuPo angehören und von dem anderen Ausdrucksmittel (aus den Klassen $\underline{M}$ oder $\underline{A}$ ) eine gerichtete Kante zu einem Mittel der Klassen A respektive Mausgeht.

So ist bei ' $x{ }^{\prime}=S / k_{2} /$ im obigen Graphen nur dann kein sicherer SchluB auf das dritte Ausdrucksmittel möglich, wenn für ' $v$ ' $M_{g}$, $A_{1.2}$ oder $A_{2.2}$ stehen. Konfigurationen dieser Art (' $x$ ' oder ' $v$ ' gehören der Klasse $P$, PuS, $S$, SuPo an) sind jedoch nur von untergeordnetem Interesse, da immer denn, wenn das Ausdrucksmittel aus der Klasse der Affixe festliegt, auch der entsprechende Graph eindeutig bestimmt ist. Dies ist neben ihrer problemlosen Erschliebbarkeit der zweite Grund dafür, daB Beziehungen dieser Art nicht eigens hervorgehoben werden sollen.

Anders verhält es sich jedoch dann, wenn weder ' $x$ ' noch ' $v$ ' der Klasse der Affixe angehören.

So prädiziert im obigen Graphen $M_{6.1}$ in Verbindung mit $A_{2} .3$ eindeutig $\mathrm{S} / \mathrm{k}_{2} /$. Mit anderen Worten, die Kombination der beiden erstgenanten Ausdrucksmittel ist ausschlieblich in Verbindung mit $S / k_{2}$, belegt. 29

Da Beziehungen dieser Art (Ausdrucksmittel aus den Klassen $\underline{M}$ und $A$ prädizieren in ihrer Verbindung eindeutig ein Mittel aus der Klasse $P$, PuS, $S$, SuPo und somit den Graphen, dem sie angehören) erheblich seltener sind als die zuvor beschriebenen und zudem nicht unmittelbar evident, verdienen sie eine besondere Kennzeichnung.

In der nun folgenden Zusammenstellung werden noch einmal sämtliche allgemeinen Explikationen der deadjektivischen Redeteile

29 Es versteht sich von selbst, daB zwischen $M_{6.1}$ und $A_{2.3}$ auch eine Beziehung vom Typ (2) vorliegt, was der besseren Ubersichtlichkeit halber graphisch jedoch nicht eigens hervorgehoben wird. 
genannt und die Kombinationen der Mittel, die zu deren Ausdruck beitragen, in der Reihenfolge geordnet, wie dies unter 2.4.1.1.1. (S. 100) bis 2.4.5.4.2.4. (S. 170) der Fall war. Die Seitenangaben bei jedem Graphen ermöglichen einen raschen orientierenden Vergleich mit der primären Deskription.

A Deadjektivische Substantive

1. Substantive mit der Bedeutung eines abstrakten Merkmals

1.1. Merkmal als gesellschaftliche, politische, wissenschaftliche oder ästhetische Erscheinung oder Tendenz (vgl.o., lo0f)

S 3

1.2. Erscheinung der auBersprachlichen Wirklichkeit, die charakterisiert ist durch das Vorliegen des mit dem ableitenden Adjektiv benannten Merkmals in abgeschwächter Form

(1) $($ vgl.0., 102)

$$
\begin{aligned}
& A_{2.2} \\
& A_{2.5}
\end{aligned}
$$

(2) (vgl.o., 102f) 
(3) (vgl.o., 103)

2.2

S

2.5

(4) (vgl.0., 103)

Pus/pro ...

.1

1.3. Merkmal als negativ bewertete Erscheinung oder Tendenz (vgl.0., 104)

S

.1

2. Substantive mit der Bedeutung des Merkmalsträgers

2.1. Gesamtheit dessen, was sich durch ein bestimmtes Merkmal auszeichnet

(1) $(v g 1.0 ., 106)$

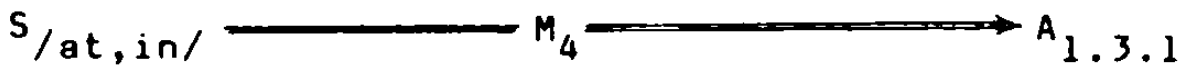

(2) $($ vgl.0., 107)

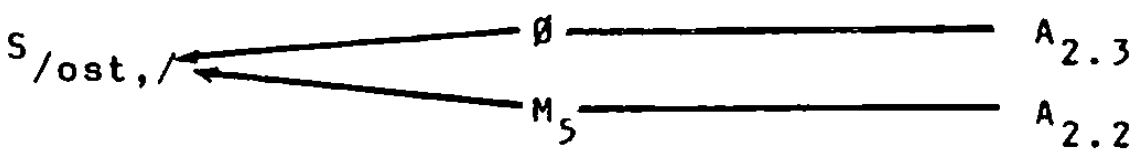


(3) $(v g 1.0 ., 107)$

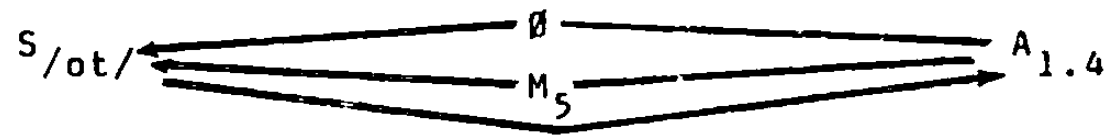

(4) (vgl.o., 107f)

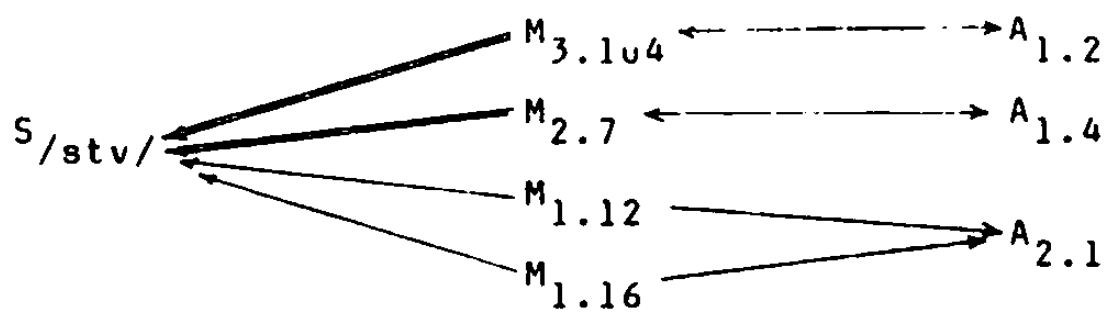

$(5.1)(v g l .0 ., 108)$

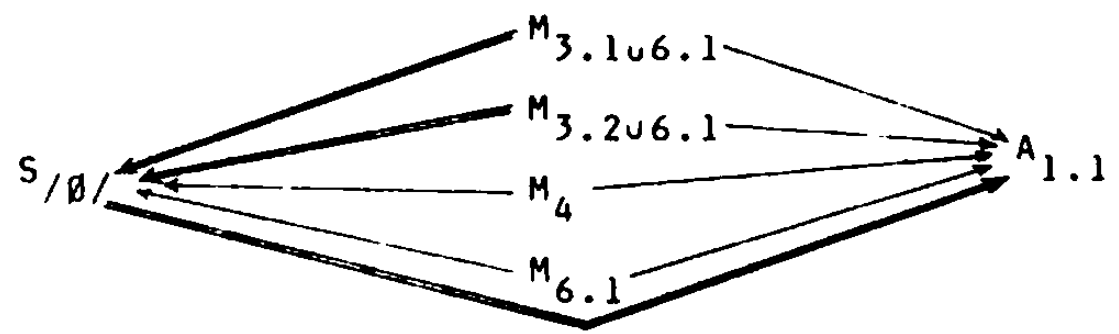

$(5.2)$ (vgl.o., 108f)

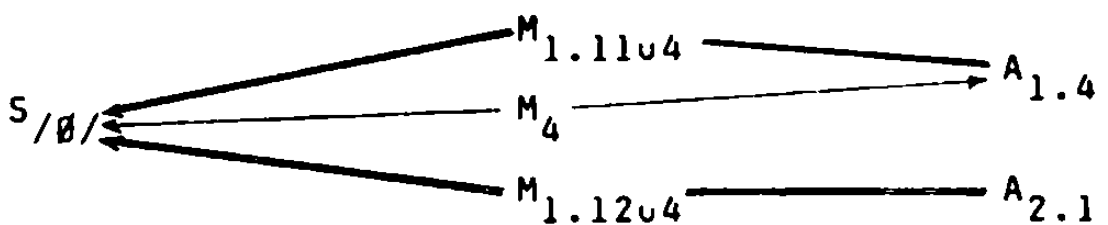

(6) (vgl.o., 109)

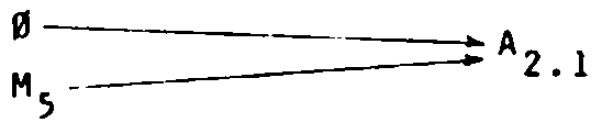


2.2. Jemand (etwas), der (das) sich durch ein bestimmtes Merkmol auszeichnet

(1) (vgl.0., 115)

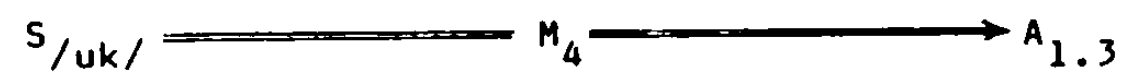

(2) (vg1.0., 115)

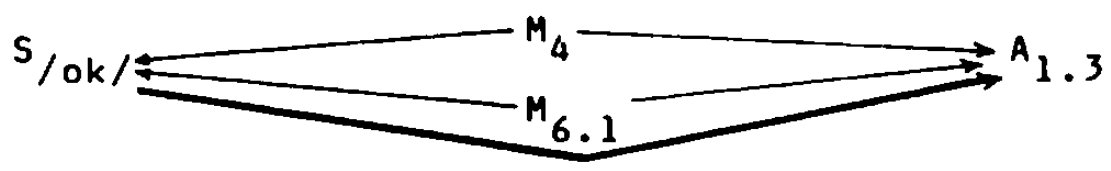

(3) (vg1.0., 115)

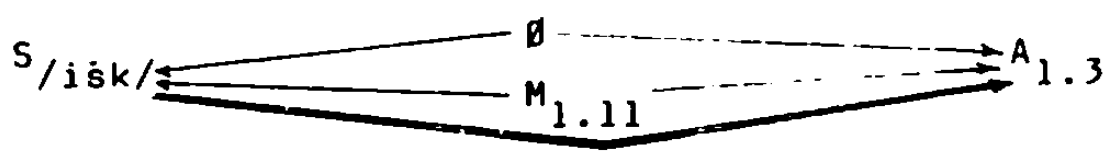

(4) $($ vgl.0., 115)

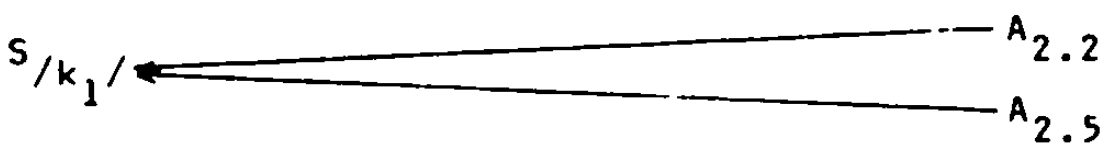

(s) $($ vgl.0., 116)

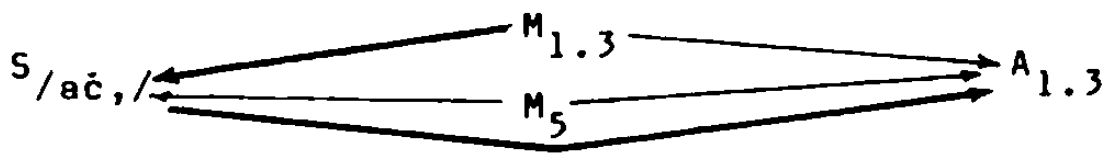

(6) $(v g 1.0 ., 116)$

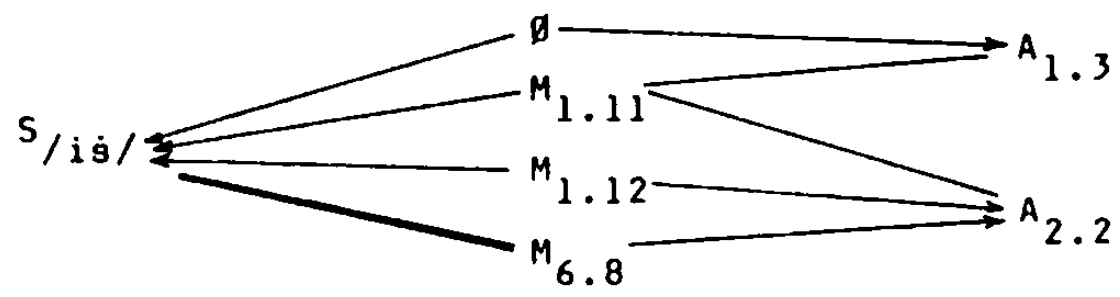


(7) (vgl.o., 116f)

S

(8) $($ vgl.0., 117)

$\mathrm{S}_{\mathrm{u} u \mathrm{x}} \longrightarrow \mathrm{A}_{1.3}$

(9) (vg1.0.,117)

S/ǘ

(10) $($ vgl.o., 118)

S

(11) (vgl.o., 118)

s/ul, 
(12) (vg1.0., 118)

M

6
.3

.1

.2

.4

(13) (vgl.o., 119)

1

2

3

2

4

4

(14) (vgl.0., 120f)

2

S

4

3

2 
(15) (vgl.0., 121)

$5 / k_{2}$

$$
.2
$$

.2

.1

$-.3$

(16) (vgl.o., 122)

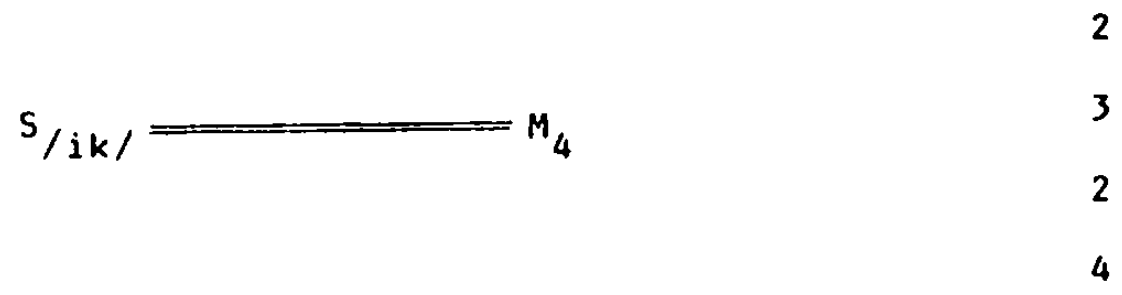

(17) (vgl.o., 122)

s/ug/

(18) (vgl.o., 123)

${ }^{A} 2.1$

S

$$
\begin{array}{r}
A_{2.2} \\
A_{2.5}
\end{array}
$$


(19) (vgl.o., 123)

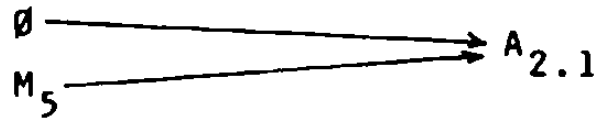

B Deadjectivische Adjektive

1. Gradation des mit dem ableitenden Adjektiv benannten Merkmals

1.1. Hervortreten des Merkmals in mäßiger Form

(1) (vgl.0., 125)

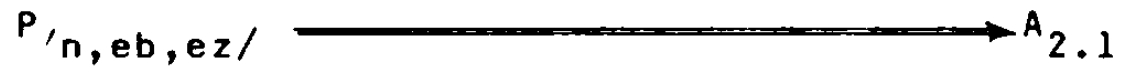

(2) (vgl.0., 125f)

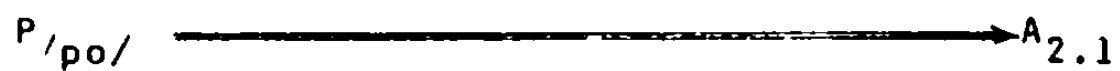

(3) (vgl.0., 126)

S

3.2

1.2. Hervortreten des Merkmals in verstärkter Form

(1) $(v g l .0 ., 127)$

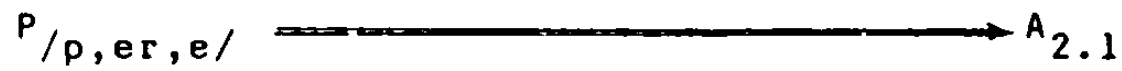

(2) (vgl.o., 127)

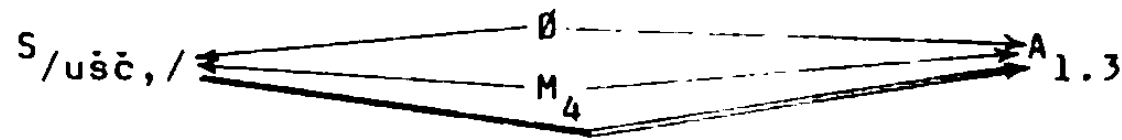


1.3. Hervortreten des Merkmals in höchster oder in übermäßiger Form
(1) $($ vgl.0., 130)

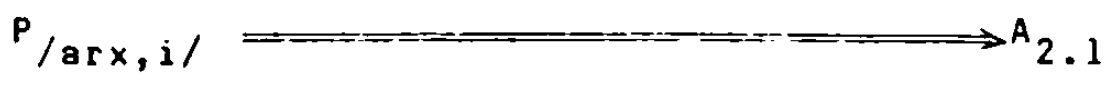
(2) (vgl.0., 130)

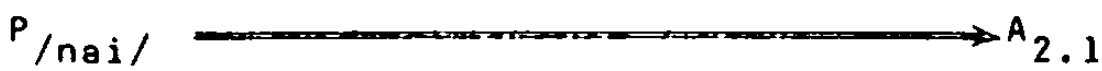
(3) $($ vgl.0., 130)

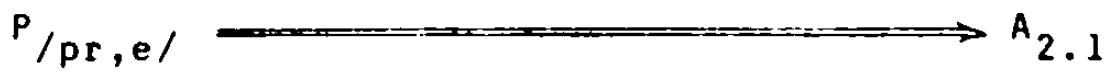

(4) $($ vgl.0., 130)

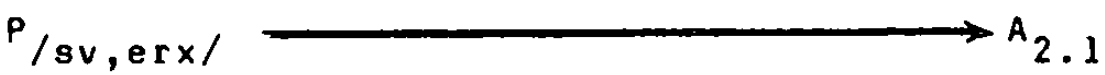

(s) (vg1.0., 131)

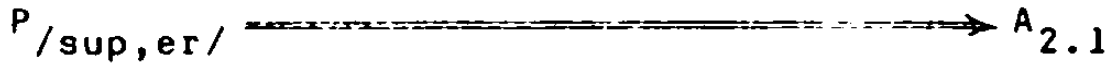

(6) (vgl.0., 131)

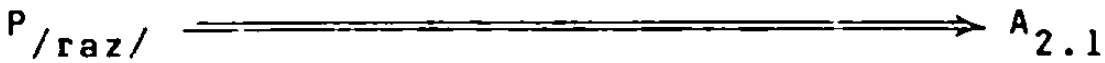

(7) $($ vgl.o., 131)

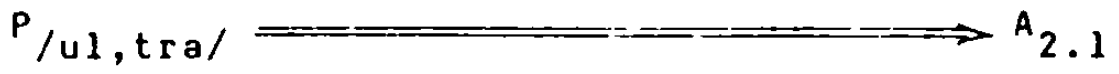

(8) (vgl.o., 131) 
(9) (vgl.0., 132)

S

(10) (vgl.o., 132f)

PuS $/ 28 \ldots$ onn $\Longrightarrow \mathrm{M}_{4} \Longrightarrow A_{1.3}$

2. Negierung oder Nichtvorhandensein des mit dem ableitenden Adjektiv bezeichneten Merkmals oder Ausdruck der Entgegengesetztheit zu diesem

(1) (vg1.0., 134)

$\mathrm{P} / \mathrm{a} / \Longrightarrow A_{2.1}$

(2) (vgl.0., 134)

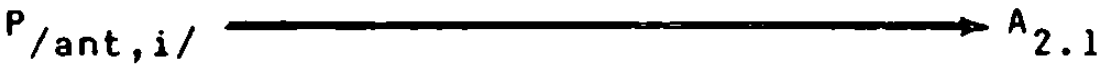

(3) (vgl.0., 134)

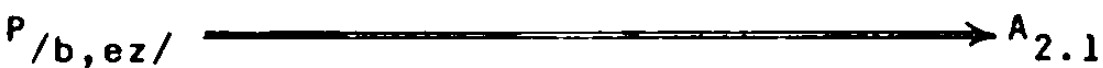

(4) $($ vgl.0., 135)

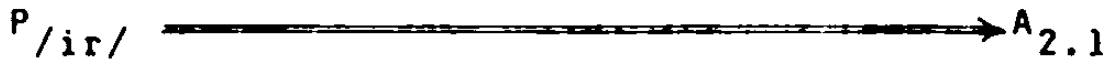

(5) (vgl.0., 135)

$P / n, e / \Longrightarrow A_{2.1}$ 

(6) (vgl.0., 135)
P/prot,ivo/ $\longrightarrow A_{2.1}$

3. Expressive Nuancierung des Merkmals und gleichzeitiges Hervortreten desselben in verstärkter form

(1) (vgl.0., 136)

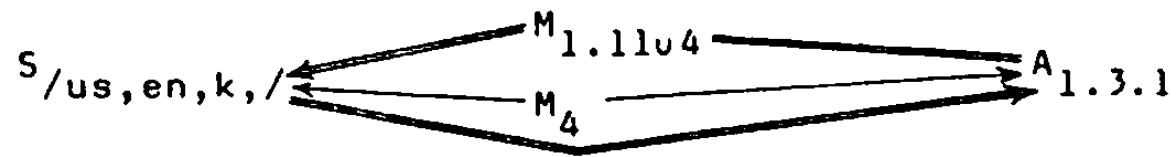

(2) (vgl.0., 137)

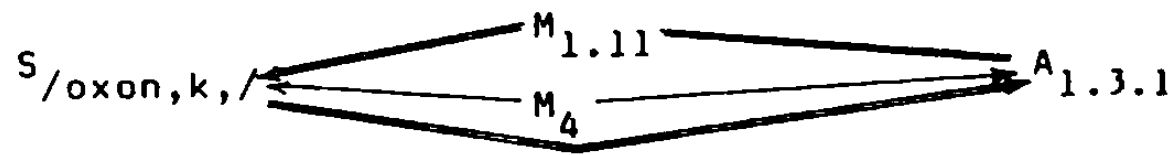

(3) (vg1.0., 137)

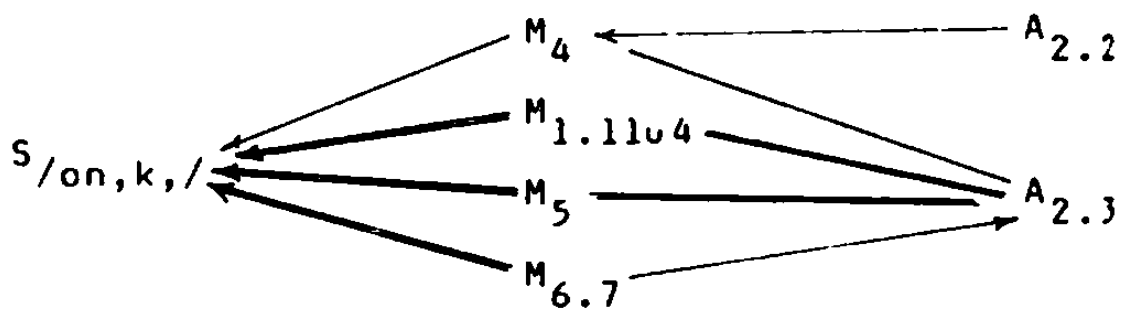


C Deadjektivische Verben

1. Das mit dem ableitenden Adjektiv benannte Merkmal annehmen oder an den Tag legen

(1) $(v g 1.0 ., 141)$

$\mathbf{S}$

(2) (vgl.0., 141f)

S

.3

.1

(3) (vgl.o., 142)

.3 
(4) (vgl.0., 143)

SuPo/i ...

(s) (vgl.o., 143f)

S

.1

(6) (vg1.0., 144)

5

(7) (vgl.o., 144f)

.3 .2

S

.2

(8) (vgl.0., 145)

S

.1 
2. Etwas (jdn.) mit dem durch das ableitende Adjektiv benannte Merkmal versehen

(1) $(v g l .0 ., 149 f)$

$\mathrm{S} / \mathrm{i}_{2}{ }^{\prime}$

4

(2) (vg1.0., 151)

(3) $(v g l .0 ., 151 f)$

S

(4) (vgl.0., 152)

PuS/vi ... i

.1 
(5) (vg1.0., 153)

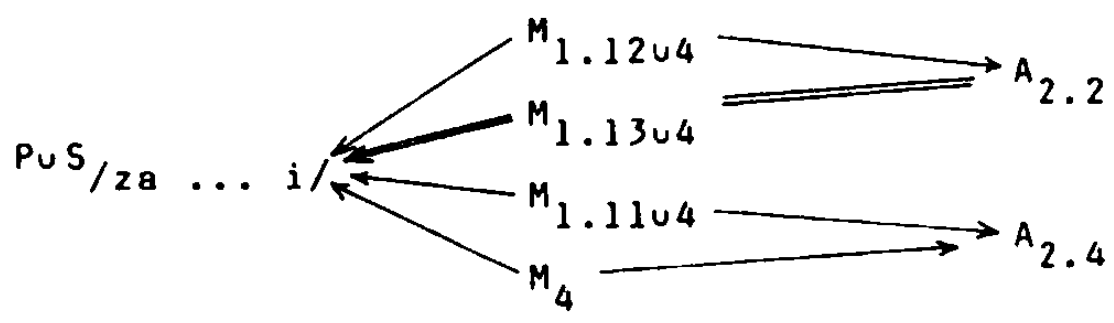

(6) (vgl.0., 153)

Pus/na $\ldots$ i / $\Longrightarrow M_{4} \Longrightarrow A_{1.2}$

(7) (vgl.0., 154)

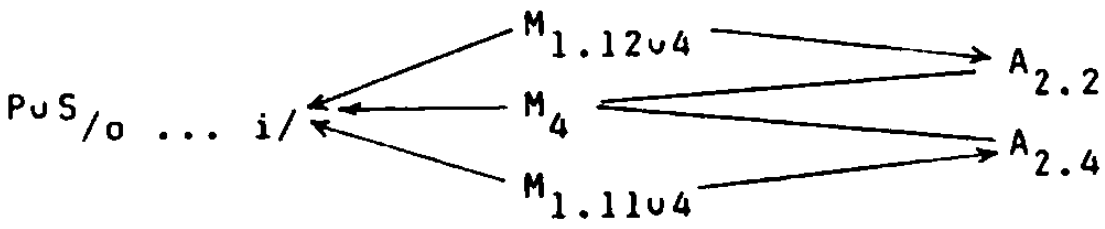

(8) (vg1.0., 154)

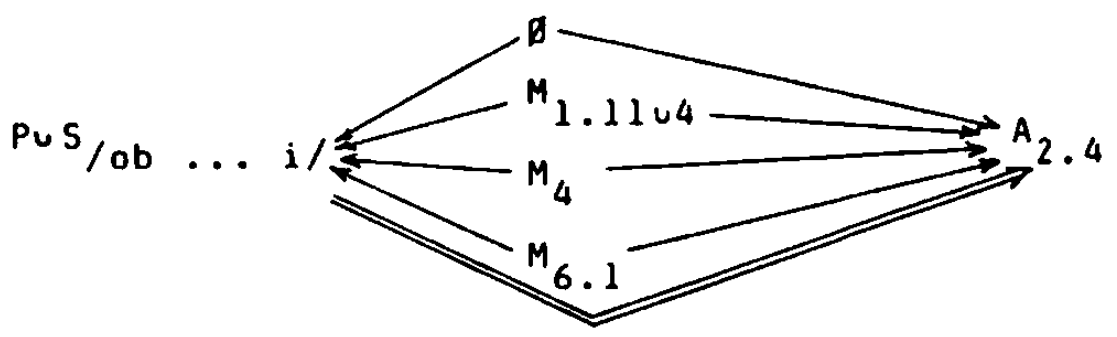

(9) (vgl.0., 155)

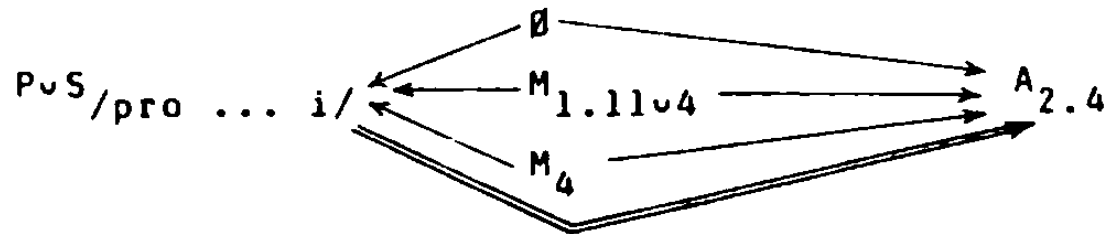


(10) (vgl.0., 155)

PuS/raz ...i i/l

.4

(11) (vgl.0., 155f)

2.2

PuS/s ... il

2.4

1.2

(12) (vgl.0., 156)

.2

$\mathrm{PuS} / \mathrm{u} . . . \mathrm{i}$

.4

(13) (vgl.0., 157)

Pus/za ...e el

.1

(14) (vgl.0., 157)

$$
\text { PuS/o .. e/ }
$$

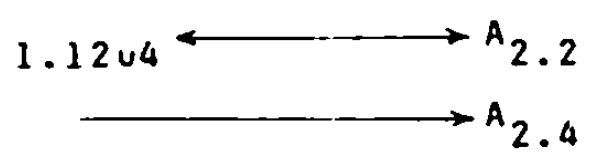

(15) (v9l.0, 157)

$$
\text { PuS/po .. e }
$$


2.1. Etwas (jdn.) in geringem MaBe mit dem durch das ableitende Adjektiv benannte Merkmal versehen

(vgl.0., 158)

$$
\text { Pus/pod }
$$

2.2. Etwas (jdn.) in stärkerem MaBe mit dem durch das ableitendes Adjektiv benannte Merkmal versehen

(vgl.o., l59f)

$$
\text { PuS/po ... }
$$

2.3. Etwas (jdn.) übermäBig mit dem durch das ableitende Adjektiv benannte Merkmal versehen

(1) $(v g 1.0 ., 161)$

$$
\text { Pus/is }
$$

(2) (vgl.o., 161)

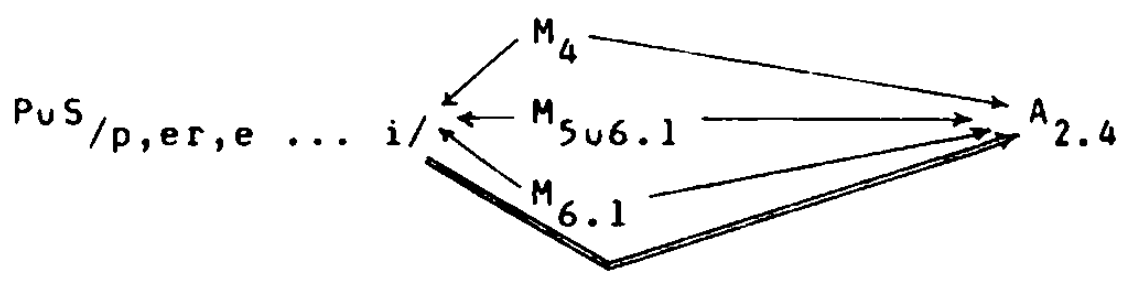


3. Etwas (jdn.) des mit dem ableitenden Adjektiv benannten Merkmals berauben

(vgl.o., 162)

$$
\text { Pus/raz } \ldots i / 2
$$

$$
\begin{array}{r} 
\pm A_{2.4} \\
A_{2.2}
\end{array}
$$

D Deadjektivische Adverbien

1. Hervorteten des mit dem ableitenden Adjektiv benannten Merkmals in verstärktem MaBe

(1) (vgl.0., 164)

$$
\text { S/im/ }
$$

(2) (vgl.0., 164f)

$$
\text { Pus/na } \ldots \text { o/ }
$$

2. Uberführung von etwas (jdn.) in einen Zustand, der sich durch das mit dem ableitenden Adjektiv benannte Merkmal auszeichnet

(1) (vgl.0., 166)

$$
\text { PuS/do ... a/ }
$$

$$
A_{1.1}
$$

$$
A_{1.3}
$$

(2) $($ vgl.o., 166)

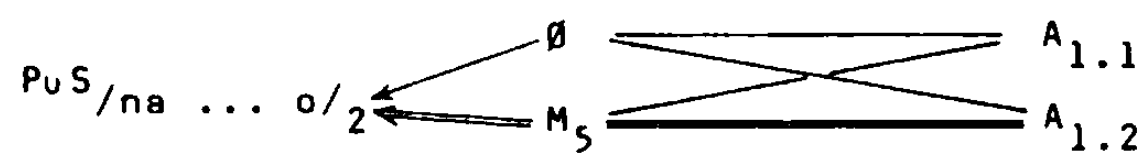


3. Gerichtetheit auf das mit dem ableitenden Adjektiv berannte Merkmal

(1) $($ vgl.0., 167)

$$
\mathrm{PuS} / \mathrm{na} \ldots \mathrm{o}_{3}=\ldots \mathrm{A}_{1.2}
$$

(2) (vgl.0., 168)

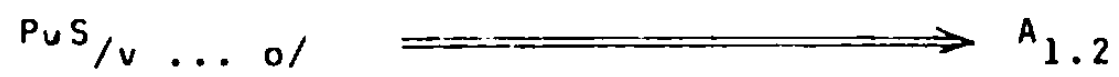

4. Seinen Anfang bei dem nehmen bzw. von dem ausgehen, wis mit dem ableitenden Adjektiv benannt ist

(1) (vgl.0., 169)

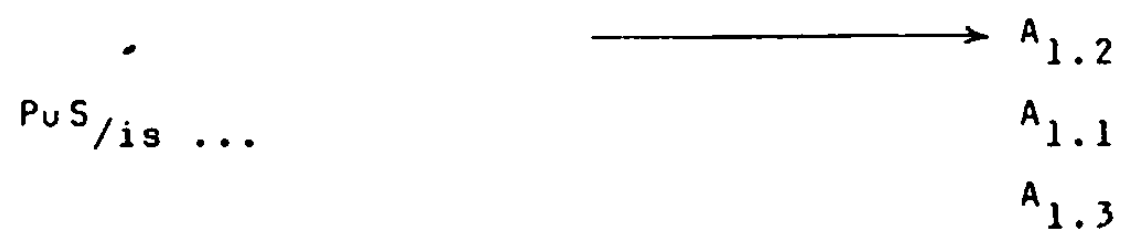

(2) (vgl.0., 169f)

$$
\text { PuS/s ... a/ }
$$

(3) $($ vgl.o., 170)

PuS/so ...

(4) (vgl.o., 170)

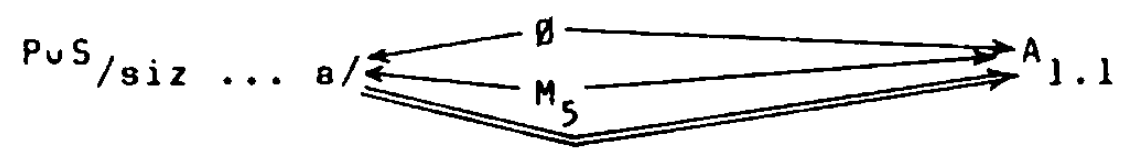


2.4.8. Analyse der Ausdrucksmittel hinsichtlich ihrer prädiktiven und ihrer diskriminatorischen Stärke

Eine Auswertung der Graphen im Hinblick auf die natürlich besonders interessierenden implikativen Regelhaftigkeiten vom Typ 4 (vgl.o., 176) führt zu recht überraschenden Ergebnissen. Betrachtet man jede einzelne der in den Graphen dargestellten Kombinationen von Ausdrucksmitteln aus den Klassen der Affixe, der morphonologischen Alternationen sowie der Akzentkonfigurationen als Modell für konkrete Bildungen, und läBt man die Anzahl der nach diesen Modellen gebildeten Realisierungen zunächst unberücksichtigt, so ist die, wie bereits angeführt, oft zumindest implizit angenommenen Hypothese von der dominierenden Rolle der Affixe bei der Prädiktion von Wortbildungsmodellen zu relativieren. Zieht man nämlich die einzelnen Klassen von Ausdrucksmitteln jeweils für sich heran, so ergibt sich folgendes Bild:

1. Im Bereich der Affixe kann dann, wenn

1.1. das Präfix bekannt ist, in sechzehn Fällen die Akzentkonfiguration prädiziert werden und damit jeweils das gesamte Modell, da keine morphonologischen Alternationen stattfinden.

1.2. das Suffix bekannt ist, in 23 fällen die Akzentkonfiguration prädiziert werden. Dabei steht in einem Fall (A 2.2. (8)) auch das entsprechende Modell insgesamt fest, da keine morphonologischen Alternationen auftreten. In zwei Fällen ( $A$ 2.1.(1) und $A$ 2.2.(1)) ist aufgrund der Kenntinis des Suffixes auBer einer Prädiktion der Akzentkonfiguration auch die der morphonologischen Alternationen möglich, so daß auch hier jeweils eindeutige Rückschlüsse auf das entsprechende Modell gezogen werden können.

1.3. die Kombination von Präfix und Suffix bekannt ist, in sechzehn Fällen die Akzentkonfiguration prädiziert werden, wobei in zwei fällen ( $3 .(1)$ und $D 3 .(2)$ ) auch das entsprechende Modell insgesamt $f$ ixiert ist, da keine morphonologischen Alternationen stattfinden. In einem Fall (C 2. (6)) ist aufgrund der Kenntnis der Affixkombination neben 
der Prädiktion der Akzentkonfiguration auch die der morphonologischen Alternationen möglich, so dab auch hier das gesamte Modell bestimmt ist.

2. Für den Bereich der morphonologischen Alternationen kann dann, wenn es sich

2.1. Um eine einzelne Alternation ( $z . B . M_{2.19}$ ) handelt,

2.1.1. in vierzehn Fällen das Suffix eindeutig bestimmt werden. Da es dabei auch immer möglich ist, die Akzentkonfiguration eindeutig zu prädizieren, sind vierzehn Modelle fixiert.

2.1.2. in zwei Fällen (D 2.(2) und $D$ 4.(2)) jeweils eine Kombination von Präfix und Suffix prädiziert werden. Da es in einem dieser Fälle ( $04 .(2)$ ) möglich ist, auch die Akzentkonfiguration eindeutig vorherzusagen, liegt ein weiteres Modell fest.

2.2. Um eine Kombination morphonologischer Alternationen (z.B. $M_{2.10 u 4)}$ handelt, in 26 Fällen das Suffix prädiziert werden. Da dabei mit Ausnahme von A 2.2.(13) (M/2.16u4 kann sich mit $A_{1.2}$ oder mit $A_{1.3}$ kombinieren) und $B 1.3 .(9)$ $\left(M_{2.8} 4\right.$ kann sich mit $A_{1.2}$ oder mit $A_{2.2}$ oder mit $A_{2.3}$ kombinieren) auch die jeweilige Akzentkonfiguration eindeutig festliegt, sind weitere 24 Modelle vorhersagbar.

3. Was die Akzentkonfiguration anbelangt, so lassen sich Aussagen der obigen Art nicht treffen, d.h., eindeutige Prädiktionen von Modellen oder auch nur einzelner Ausdrucksmittel aus der Klasse der Affixe oder aus der der morphonologischen Alternationen aufgrund der Kenntnis einer Akzentkonfiguration sind nicht möglich.

Von einer dominierenden Rolle der Affixe im Rahmen der deadjektivischen Wortbildung kann also dann, wenn es um implikative Regelhaftigkeiten vom Typ 4 geht, nicht die Rede sein, da es aufgrund der Kenntnis der entsprechenden Affixe gelingt, 22 Modelle (7.9\% aller Modelle) zu prädizieren, es jedoch auf der Basis einer Kenntnis der morphonologischen Alternationen möglich ist, 39 Modelle (also 14\% aller Modelle) zu bestimmen. 
Insgesamt sind demnach ca. $22 \%$ aller für die deadjektivische Wortbildung des Russischen konstitutiven Modelle ouf der Grundlage der Kenntnis jeweils nur eines Ausdrucksmittels aus den Klassen der Affixe oder der morphonologischen Alternationen voraussagbar.

Wie oben ( 5.177 ) bereits angedeutet, ändern sich die Verhältnisse dann, wenn innerhalb eines Modells zwei Ausdrucksmittel als bekannt vorausgesetzt werden. Die Anzahl dieser implikativen Regelhaftigkeiten vom Typ ' $x \longrightarrow v \longrightarrow y$ ', die in den Graphen jeweils dann kenntlich gemacht sind, wenn weder ' $x$ ' noch ' $v$ ' der Klasse der Affixe angehören, steht (und dies zeigt bereits eine oberflächliche Überprüfung der Graphen) deutlich hinter den Regelhaftigkeiten dieses Typs zurück, die sich dann ergeben, wenn für ' $x$ ' oder für ' $v$ ' ein Element aus der Klasse der Affixe eingesetzt wird.

Die obigen Ergebnisse bedürfen dann einer weiteren Relativierung, wenn es nicht mehr nur um Modelle, sondern um konkrete Realisierungen dieser Modelle geht. Zwar ist es möglich, allein auf der Basis der morphonologischen Alternationen 14\% aller Modelle der deadjektivischen Wortbildung zu prädizieren, doch wird nach diesen Modellen - infolge der systemischen Singularität der in Rede stehenden Alternationen - lediglich ein Bruchteil der tatsächlichen Bildungen realisiert.

In funktioneller Hinsicht fält den Affixen ohnehin die dominierende identifikatorische bzw. diskriminatorische Stärke zu. So können bei Kenntnis des Affixes bzw. der Affixkombination, von wenigen Ausnahmen abgesehen (dann, wenn es sich um Affixe handelt, die hinsichtlich ihres Signifikanten identisch und in unterschiedlichen Graphen vertreten sind (vgl. $2.8 .5 /$ in/ in $A$ 1.2.(1) und in A 2.2.(13)), oder dann, wenn affixlose Derivation vorliegt (vgl. 2.8. A 2.1.(6) und A 2.2. (19)), eindeutige Rückschlüsse sowohl auf den abgeleiteten Redeteil wie auch auf dessen allgemeine Explikation gezogen werden. Dies ist mit Blick auf die morphonologischen Alternationen nur dann möglich, wenn sie, wie bereits ausgeführt, in ihrer Ausprägung systemisch singulär sind. Die Akzentkonfigurationen schlieblich entbehren, je für sich genommen, jeglicher diskriminatorischer Stärke. 
2.4.9. Darstellung der Korrelationen zwischen den Derivatemtypen und den Graphen

Die an der deadjektivischen Wortbildung des Russischen potentiell beteiligten Derivateme lassen sich zu Typen zusammenfassen, die aus der oben (77-83, 85f) vorgenommenen Zerlegung der offenen Menge der Adjektive auf der Grundlage der allgemeinen Explikationen ihrer Inhaltselemente resultieren. Im folgenden soll es darum gehen, die bei der Ableitung präferierten Derivatemtypen so kenntlich zu machen, daß jeder Graph, innerhalb dessen, wie bereits erläutert, jede einzelne Kombination von Ausdrucksmitteln aus den Klassen der Affixe, der morphonologischen Alternationen sowie der Akzentkonfigurationen als Modell für konkrete Bildungen gelten kann, mit denjenigen Derivatemtypen korreliert wird, deren konkrete Ausprägungen (= Angehörige) an der Realisierung der jeweiligen Modelle beteiligt sind.

Die insgesamt 278 Modelle der deadjektivischen Wortbildung sind zu 94 Graphen zusammengefaBt, von denen 32 den Bereich der Bildung von Substantiven, 24 den der Bildung von Adjektiven, 28 den der Bildung von Verben und zehn den der 8 ildung von Adverbien abdecken. Diesen 94 Graphen stehen insgesamt 50 Derivatemtypen gegenüber (nichtabgeleitete Adjektive (1), desubstantivische Adjektive (29), deverbale Adjektive ( 8 ), denumerale Adjektive (2), Adjektive, die als aus den Stämmen von zwei oder mehr Lexemen gebildete Komposita gedacht werden können (5) und deadjektivische Adjektive (5)).

Um die tabellarische Darstellung nicht übermäBig auszuweiten, werden nur diejenigen (insgesamt 18) Derivatemtypen aufgelistet, deren Angehörige auch tatsächlich an der deadjektivischen Wortbildung beteiligt sind. Die am untersuchten Korpus nicht belegten Jypen erscheinen in der Tabelle als Auslassungen und können auf den Seiten 78-83 sowie 85f nachgeschlagen werden.

Am Beispiel des Graphen B 1.1.(3) (vgl.0., 185) sei verdeutlicht, wie die unten zusammengestellte Tabelle zu lesen ist: An der Bildung von Adjektiven mit der allgemeinen Explika- 
tion 'Hervortreten des mit dem ableitenden Adjektiv benannten Merkmals in mäBiger Form' sind Angehörige folgender Derivatemtypen beteiligt: I (nichtabgeleitete Adjektive), II/l (desubstantivische Adjektive, die das mittels inrer spezifizierte Denotat kennzeichnen als eine Eigenschaft aufweisend, die dem mit dem ableitenden Substantiv Benannten zugeschrieben wird) und III/7 (deverbale Adjektive, die das mittels ihrer spezifjzierte Denotat als in einem Zustand befindlich kennzeichnen, der als Resultat der mit dem ableitenden Verb benannten Handlung entstanden ist). Diese allgemeinen Explikationen erfahren durch die im Graphen abgebildeten Kombinationen von Ausdrucksmitteln aus den Klassen der Affixe (S/ovat/), der morphonologischen Alternationen $\left(M_{\emptyset}\right.$ bzw. $M_{1.14}$ ) und der Akzentkonfigurationen ( $A_{1.3 .2}$ ) Veränderungen sowohl auf der Ausdrucks-wie auch ouf der Inhaltsseite. 
- sor - 
Die in tabellarischer form dargestellten Beobachtungen lassen sich verbal wie folgt zusammenfassen:

In 87 Graphen (das sind $92.5 \%$ aller Graphen) kann oder muB (letzteres insbesondere im Rahmen der deadjektivischen Bildung von Adverbien) bei der Realisierung von Wortbildungsmodellen ein nichtabgeleitetes Adjektiv als Derivatem fungieren. Die sieben Graphen, in denen keine Ausprägungen dieses Derivatemtyps zur Umsetzung der entsprechenden Modelle herangezogen werden, entfallen auf den Bereich der deadjektivischen Bildung von Substantiven ( 1 1.1., A 1.3. und $A$ 2.1.(4)) sowie auf den der deadjektivischen Bildung von Adjektiven (B 1.1.(1), B 1.2.(1), $B$ 1.3.(2) und $B$ 2.(1)). Bemerkenswert ist, daB in der Mehrzahl der genannten Fälle ganz ausschlieBlich Ausprägungen des Typs II/I genutzt werden. Bei B 1.2.(1) können auch Angehörige des Typs III/7 als Derivateme auftreten, und lediglich das im Graphen B 1.3.(2) repräsentierte Modell nimmt insofern eine Sonderstellung ein, als es das einzige aller 278 Modelle ist, an dessen Realisierung weder Derivateme vom Typ I noch solche vom Typ II/l mitwirken.

Dementsprechend hoch ist auch der Anteil der Graphen, deren Modelle unter Rückgriff auf Derivateme des Typs II/l realisiert werden ( 52 Graphen oder 55.3\% aller Graphen). Die übrigen Derivatemtypen spielen mit Ausnahme von $111 / 7$, dessen Ausprägungen zur Verwirklichung von Modellen in insgesamt achtzehn Graphen (19.1\%) herangezogen werden, eine eher marginale Rolle. So sind in bezug auf die Anzahl der Graphen, innerhalb derer sie an der Realisierung von Modellen mitwirken, die deadjektivischen und die als Komposita aufzufassenden Adjektive als Derivateme von untergeordneter Bedeutung; am wenigsten genutzt werden denumerale Adjektive, die nur bei zwei Graphen (2.1\% aller Graphen) als Derivateme zur Umsetzung von Wortbildungsmodellen fungieren. Neben Angehörigen der Derivatemtypen I und/oder $1 \mathrm{I} / \mathrm{l}$ können solche anderer Typen im Rahmen der deadjektivischen Wortbildung (a) von Substantiven bei dreizehn Graphen zur Realisierung von Modellen herangezogen werden (40.6\% der Graphen in diesem Bereich), (b) von Adjektiven bei acht Graphen (33.3\%), (c) von Verben bei sieben Graphen (25\%) und (d) von Adverbien bei vier Graphen $(40 \%)$. 
Zusammenfassend lassen sich so bei der deadjektivischen Wortbildung hinsichtlich der verwendeten Derivateme folgende Präferenzen feststellen: Der am stärksten genutzte Typ umfaBt Adjektive, die als nichtabgeleitet aufzufassen sind, mit Abstand gefolgt von desubstantivischen Adjektiven, vorzugsweise mit der allgemeinen Explikation $11 / 1$ ( 57 Graphen). Danach rangieren deverbale Adjektive ( 24 Graphen), wobei solche mit der allgemeinen Explikation III/7 dominieren, gefolgt von solchen Adjektiven, die als aus den Stämmen von zwei oder mehr Lexemen gebildete Komposita gedacht werden können (zehn Graphen). Wiederum in bezug auf die Anzahl der Graphen, bei denen sie als Derivateme zur Realisierung von Wortbildungsmodellen fungieren, folgen etwa gleichauf (neun Graphen) deadjektivische Adjektive, und den Schlub bilden, wie bereits angeführt, die denumeralen Adjektive. 
3. ZUSAMMENFASSUNG UND BEWERTUNG DER LEISTUNG DER METHODE

Den heuristischen Rahmen für die empirische Untersuchung der deadjektivischen Wortbildung des Russischen bildete die sogenannte 'analytisch-synthetisch-funktionelle' Methode zu Deskription der Formenbildung der slavischen Sprachen. Was die Leistung dieser Methode anbelangt, so können (wie bereits mehrfach hervorgehoben), vor allem aufgrund der Unterschiede hinsichtlich der Inhaltsseiten der untersuchten Wortformen, die Ergebnisse in unserem Bereich nicht ganz den im Rahmen der Formenbildung erzielten entsprechen, worauf am Ende dieser Zusammenfassung noch näher einzugehen ist. Zunächst sei jedoch der Gang der Untersuchung noch einmal kurz skizziert.

In einem ersten grundlegenden Schritt ging es darum, die im Rahmen der empirischen Analyse zu verwendende Begrifflichkeit in sich kohärent zu bestimmen. Lediglich erläutert oder exemplifiziert wurden dabei solche Begriffe, die als hinreichend bestimmt oder unter vielen (bzw. all ihren) Aspekten beschrieben gelten können; definiert wurden dagegen alle Begriffe, die für die Wortbildung als linguistische Disziplin in besonderem Maße spezifisch sind, wobei der neuere stand vor allem der sowjetischen forschung aufgearbeitet wurde.

Die empirische Analyse ging von der Prämisse aus, daB es sich bei dem Gegenstand der Wortbildung als linguistischer Disziplin um das 'fertige' Wort handelt, auf das eine strukturelle Analyse anzuwenden ist, und setzte ein mit einer klassifikation der Gesamtheit der Adjektive des Russischen auf der Grundlage ihrer nichtgrammatischen Inhaltsseiten, an die sich eine ähnlich geartete Darstellung der Inhaltsseiten der deadjektivischen Redeteile anschloß. Die Orientierung an den nichtgrammatischen Inhaltselementen resultierte daraus, daB die Derivation als ein Verfahren angesehen werden kann, das darauf zielt, nichtgrammatische Inhaltselemente von Lexemen aus kommunikativen Erfordernissen heraus auf bestimmte Weise in anderen Lexemen zu modifizieren. Einzuräumen ist allerdings, dab die Prozedur, mit der jeweils solch eine allgemeine Explikation ermittelt wird, die allen individuellen Bedeutungen der jeweils untersuchten Lexeme nicht widerspricht, aus den oben dargelegten 
Gründen nicht operationalisiert werden kann (es gibt, obgleich sie zu den vordringlichsten Desiderata zählt, bis heute noch keine systematisch befriedigende und vom Sprachgefühl des Linguisten unabhängige Wortbildungssemantik), so daß die in diesem Teil der Arbeit vorgestellten Ergebnisse unter Umständen nur eingeschränkt intersubjektiv nachvollziehbar sind. Besonders hervorzuheben ist jedoch, dab die hier befolgte Vorgehensweise eine grundsätzlich andere Ordnung der Beschreibung ermöglichte, als dies allgemein üblich ist. Sieht man nämlich die Wortbildung als ein Verfahren zur Modifikation der nichtgrammatischen Inhaltselemente von Lexemen an, so hat sich eine Deskription solcher Verfahren folgerichtig an den 'Wortbildungsbedeutungen' $z u$ orientieren, denen dann die entsprechenden Bildungsmodelle zuzuordnen sind, und nicht umgekehrt.

Erst nach diesem Schritt (von der ganzheitlichen form zum Inhalt) konnte es darum gehen, die Ausdrucksmittel je für sich zu beschreiben, die in Verbindung mit den Basisformen der ableitenden Adjektive die zuvor ermittelten nichtgrammatischen Inhaltselemente der deadjektivischen Redeteile anzeigen, also die Affixe, die morphonologischen Veränderungen der Bezugsform und schlieBlich die Akzentkonfigurationen.

Diese zunächst isoliert betrachteten Ausdrucksmittel wurden dann wieder synthetisiert, um deutlich zu machen, welche Iypen von Basisformen mit welchen dieser Mittel zur deadjektivischen Derivation von Redeteilen mit den zuvor genannten Inhaltselementen kombiniert sind. Dabei ging es jedoch nicht nur um eine reine Deskription dessen, was tatsächlich beobachtbar ist, sondern vielmehr auch darum, aufzuzeigen, welche Kombinationen das Russische maximal verwirklichen kann und welche es minimal ausnutzen könnte.

Diese Art der Darstellung ermöglichte es, unter Rückgriff auf das 'MaB der Verbundenheit' die implikativen Beziehungen zwischen den einzelnen Ausdrucksmitteln zu berechnen und diese - beispielsweise mit den für andere Sprachen zu ermittelnden - vergleichbar zu machen. Dem Ergebnis dieser Berechnung zufolge kommt das Russische im Bereich der deadjektivischen Wortbildung dem maximalen Grad der Verbundenheit recht nahe. Diese implikativen Regelhaftigkeiten, die in insgesamt fünf Ty- 
pen unterteilt wurden, graphisch zu verdeutlichen und auf dieser Grundlage die Ausdrucksmittel hinsichtlich ihrer prädiktiven und ihrer diskriminatorischen Stärke zu analysieren, war die Aufgabe des nächsten Kapitels. Das interessanteste Ergebnis dieser Untersuchung bestand darin, daB dann, wenn man nur die Modelle und nicht die Anzahl der nach ihnen gebildeten Realisierungen ins Auge faBt, nicht den Affixen, sondern den morphonologischen Alternationen die dominierende Rolle bei der Prädiktion von Wortbildungsmodellen zufällt. Bezieht man jedoch die Anzahl der nach den jeweiligen Modellen gebildeten Lexeme ein und berücksichtigt auBerdem, daB dann, wenn das Ausdrucksmittel aus der Klasse der Affixe festliegt, in der Regel eindeutige Rückschlüsse sowohl auf das abgeleitete Redeteil wie auch auf dessen allgemeine Explikation möglich sind, so kommt zweifelsfrei den Affixen die höhere identifikatorische bzw. diskriminatorische Stärke zu.

Der letzte Schritt der Arbeit bestand darin, die Derivatemtypen mit den Graphen zu korrelieren. Dabei wurde nachgewiesen, daß von den insgesamt fünfzig Derivatemtypen nur achtzehn an der deadjektivischen Derivation beteiligt sind. Wie aus der Tabelle leicht ersichtlich, ergeben sich eindeutige Präferenzen für nichtabgeleitete und für desubstantivische Derivateme, wohingegen die übrigen eine eher untergeordnete Rolle spielen. Mögliche Gründe dafür lassen sich vermutlich nur auf der Grundlage einer stringenten ( $d . h$. operationalisierten) semantischen Analyse angeben, ein Unterfangen, das im Rahmen dieser Arbeit aus den genannten Gründen nicht geleistet werden konnte.

Bevor es nun darum gehen soll, der eingangs angedeuteten Frage nach der Leistung der gewählten Methode auf den Gebieten der formen- und der Wortbildung nachzugehen, sei das Erreichte in einem Satz zusammengefaBt: Im AnschluB an eine in sich stimmige klärung der zugrunde zu legenden Begrifflichkeit gelang, ausgehend von nachprüfbaren sprachlichen Beobachtungsdaten, eine erschöpfende und weitgehend übersichtliche analytisch-synthetische Beschreibung der deadjektivischen Derivation des Russichen, aufgrund derer schlieblich ein Einblick in die Regularitäten bezüglich der Auftretensbedingungen der zuvor beschriebenen Einheiten möglich wurde. 
Diese auf der Basis der gewählten Heuristik ermittelten Regelhaftigkeiten sind jedoch von deutlich anderer qualitä als die bei der Beschreibung der Formenbildung konstatierten Regularitäten. So hat es sich im letztgenannten Bereich als ohne weiteres möglich herausgestellt, aus den aufgefundenen Regularitäten Gesetzmäßigkeiten in Form prädikativer Regeln abzuleiten. Ein solches Unterfangen wäre in bezug auf die Wortbildung mit ungleich größeren Schwierigkeiten verbunden, und dies aus folgenden Gründen:

Wie bereits mehrfach erwähnt, resultiert die gravierende Differenz bezüglich der Regularität von formen- und Wortbildung vor allem aus den Unterschieden hinsichtlich der zu untersuchenden Inhaltselemente der jeweils betrachteten Einheiten. Im Gegensatz zur Formenbildung haben wir es bei der wortbildung nicht mit einer genau definierten und auf ein kleines Korpus begrenzten Anzahl grammatischer Bedeutungen (und deshalb auch nicht mit wohldefinierten Inhalts- (und Ausdrucks-)paradigmen zu tun, sondern mit einem höchst komplexen System nichtgrammatischer Bedeutungen, denen keineswegs jeweils eindeutig bestimmte Ausdrucksseiten zugeordnet sind. In diesem Zusammenhang ist besonders hervorzuheben, daB sich (wie bereits im Haupteil der Arbeit bei der Definition der 'Wortbildungsbedeutung' erläutert), anders als bei der formenbildung, die $8 e-$ deutung des abgeleiteten Wortes nicht als 'additive' Funktion der Bedeutungen der es konstituierenden Morpheme denken läBt. Die vorliegende Arbeit trug diesem Umstand heuristisch insofern Rechnung, als eine Schrittfolge vom ganzheitlichen Inhalt über die ganzheitliche form zu den diese form konstituierenden Teilen und schlieblich wieder zum 'fertigen' Wort eingehalten wurde.

Die Untersuchung hat gezeigt, daB in keinem $F a l l$ je einer allgemeinen Explikation eines abgeleiteten Redeteils auch jeweils nur ein Wortbildungsmodell entspricht, was für die linguistische Regelfindung einen erheblichen Unsicherheitsfaktor darstellt. So lassen sich etwa für denjenigen, der Russisch als Fremdsprache erlernt, praktikable Derivationsregeln relativ mühelos nur für Realisierungen solcher Modelle erstellen, die einem Graphen angehören, der für sich allein (also nicht neben an- 
deren Graphen) der allgemeinen Explikation eines Redeteils zugeordnet ist (vgl. A 1.1. (178), A 1.3. (179), C 2.1. (194), C 2.2. (ebd.) und C 3. (195)).

Die genannte linguistische Unsicherheit entspricht jedoch keineswegs einer sprachlichen Unsicherheit, da der kompetente Sprecher des Russischen ohne weiteres in der Lage ist, je nach den kommunikativen Erfordernissen bestimmte Wörter von bestimmten anderen abzuleiten. Dieser Umstand könnte damit zusammenhängen, daß nach PLANK (1981, 25ff) nicht nur die 'Worttildungsbedeutungen' mental ganzheitlich gespeichert und bei Bedarf holistisch reproduziert werden, sondern, mit Abstrichen, auch die komplexen Formen zum Ausdruck dieser Bedeutungen, wohingegen im Rahmen der flexion das Ganze (die 'fertige' Wortform) weitgehend kompositionell aus seinen Teilen erzeugt wird. Macht man sich diese durch entsprechende Versuche zumindest in Ansätzen verifizierte Hypothese zu eigen, so korrespondieren die unterschiedlichen Regularitätseigenschaften von Wort- und Formenbildungsprozessen unterschiedlichen Verfahren ihrer mentalen Speicherung. 


\section{TERATURVERZE I CHNIS}

ACHMANOVA, 0.5.

1966 Фонология, морфонология, морФология. Москва.

ADMONI, V.G.

${ }^{3} 1970$ Der deutsche Sprachbau. München.

$A G-53 / 54$

1953/54 Академия наук СССР - Институт языкознания: Грамматика русского языка. Москва.

$A G-70$

1970 Академия наук СССР - Институт русского языка: Грамматика современного русского литературного языка. Москва.

$A G-80$

1980 Академия наук СССР - Институт русского языка: Русская грамматика. Москва.

AL TMANN, G., LEHFELDT, W.

1980 Einführung in die quantitative Phonologie. Bochum.

ANDREEV, N.D.

1967 Статистико-комбинаторные методы в теоретическом и прикладном языковедении. Ленинград.

APRESJAN, JU.D.

1969а Толкование лексических значений как проблема теоретической семантики. Известия АН СССР, Серия литературы и языка 28, I969/I, II-23.

$1969 \mathrm{~b} 0$ языке для описания значений слов. Известия АН СССР, Серия литературы и языка 28, I969/5, 415-428.

1974 Лексическая семантика. Москва.

ARDENTOV, 8.P.

1979 Фонология современного русского литературного языка. Кишинев.

ARMSIRONG, D.

1979 The study of word-formation in Russian: A survey in perspective. ORBIS XXVIII, 2, 331-364.

ARUTJUNOVA, N.D.

1961 Очерки по словообразованию в современном испанском языке. москва. 
AŠNIN, F.D. et al. (sost.)

1965 0бщее языкознание. Библиографический указатель литературы, изданной в СССР С I9I8 по I962 Г. Москва.

AVANESOV, R.I.

1974 Русская литературная и диалектная фонетика. Москва.

AVANESOV, R.I., SIDOROV, V.N.

1945 Очерк грамматики русского литературного языка. Ч. I. москва.

AVILOVA, N.S. et al. (sost.)

1954 Библиографический указатель литературы по русскому языкознанию. I825-1880. Москва.

BARTOŠEVIČ, A.

1972 К определению системы словообразования. Вопросы языкознания I972/3, 83-89.

BENSE, M.

1971 Systemtheoretische Erweiterungen des Zeichenbegriffs. Lili - Zeitschrift für Literaturwissenschaft und Linguistik 172, 1971, 91-95.

BERGER, T .

1981 Die Präsensformenbildung der tschechischen Schriftund der Umgangssprache. Kwartalnik neofilologiczny $28,1981 / 1,55-83$.

BERNŠTE JN, S.B.

1968 Введение в славянскую морфонологию. Вопросы языкознания $I 968 / 4,43-49$.

BIELFELDT, H.H. et al.

${ }^{9} 1972$ Russisch-deutsches Wörterbuch. Berlin.

BODUÉN DE KURTENE், I.A.

1963 Избранные труды по общему языкознанию. Т. I. Москва. BUDAGOV, R.A.

1958 Введение в науку о языке. Москва.

BULYGINA, T.V.

1977 Проблемы теории морфологических моделей. Москва.

BYKOVA, L.A.

1974 Современный русский литературный язык. Морфемика и словообразование. Харьнов. 
CARENKO, E.I.

19790 принципах разграничения флексий и аффиксов. Известия АН СССР, Серия литературы и языка $38,1979 / 5,425-435$.

CEREPANOV, M.V.

1972 К понятию динамического аспекта синхронного словообразования. Актуальные проблемы русского словообразования I. Самарканд, 43-49.

CHOCHLACEVA, V.N.

1973 Некоторые вопросы теории словообразования. Вопросы языкознания I973/I, 99-I04.

1976 Словообразование существительных в русском языке. АДД. Москва.

CURGANOVA, V.G.

1973 Очерк русской морФонологии. Москва.

DEBEC, N.P. et al. (sost.)

1958 Библиографический указатель литературы по языкознанию, изданной в СССР с 1918 по I957 г. Вып. І. Москва.

1969 Славянское языкознание. Библиографический указатель I96I-I965. Москва.

1973 Славянское язнкознание. Библиографический указатель I966-1970. Москва.

DOKULIL, M.

1962 Tvoŕeni slov v čes̀tinè. Praha.

1968 Zur Theorie der Wortbildung. Wissenschaftliche Zeitschrift der Karl-Marx-Universität-Leipzige Gesell203-211.

DRESSLER, $\boldsymbol{W}$.

1970 Modelle und Methoden der Textsyntax. Folia linguistica $4,1970,64-71$.

ERBEN, J.

1964 Deutsche Wortbildung in synchronischer und diachronischer Sicht. Wirkendes Wort 14, 1964/2, 83-93.

1975 Einführung in die deutsche Wortbildungslehre. Berlin. ERMAKOVA, O.P.

1972 О некоторых закономерностях в расщеплении структурной и семантической мотивации производного слова. Актуальные проблемы русского словообразования I. Самарканд, б8-72. 
FILIN, F.P.

1970 0бзор работ по современному русскому литературному языку за I966-I969 гг. Словообразование. Москва.

1978 0бзор работ по современному русскому литературному языку за 1970-I973 гг. Словообразование. Москва.

FLE ISCHER, W.

${ }^{3} 1974$ Wortbildung der deutschen Gegenwartssprache. Leipzig.

GASPAROV, B.M.

1971 Построение модели формальных классов слов современного русского языка. Труды по русской и славянской филологии XVII, Серия лингвистическая. Тарту, 63-95.

1975 Принципы построения морфологической классификации слов. Труды по русской и славянской филологии XXIII, Серия лингвистическая. Тарту, 64-93.

GAUGER, H.M.

1971 Durchsichtige Wörter. Zur Theorie der Wortbildung. Heidelberg.

GINZBURG, E.L.

$1977 K$ разработке критериев направления производности. Грамматика и норма. Москва, 83-9I.

1978 Одноименность однокорневых производных. Проблемы структурной лингвистики I976. Москва, 34-I36.

1979 Словообразование и синтаксис. Москва.

GLEJBMAN, L.V.

1972 Словообразование и формообразование в аппликативной грамматике. Проблемы структурной лингвистики I97I. москва, 2I3-244.

GOLOVIN, B.N.

1966 Приставочное внутриглагольное словообразование в русском литературном языке. Горький.

GRUCZA, F .

1970 Sprachliche Diakrise im Bereich der Ausdrucksebene des Deutschen. Beiträge zur allgemeinen Sprachtheorie. Poznań.

HENZEN, W.

${ }^{3} 1965$ Deutsche Wortbildung. Tübingen.

IL'INA, N.E.

1980 Морфонология глагола в русском языке. Москва. 
JJACHNOW, H.

1978 Wortbildung und ihre Modellierung anhand des serbokroatischen Verbalbereiches. Wiesbaden.

JJANCENECKAJA, M.N.

1979 Семантические вопросы теории словообразования. Томси.

JJANKO-TRINICKAJA, N.A.

1963 Закономерность связей словообразовательного и лексического значений в производных словах. Развитие современного русского языка. Москва, 83-97.

1968 Членимость основы русского языка. Известия АН СССР, Серия литературы и языка $27,1968 / 6,532-540$.

KKAL TWASSER, J., KEMPGEN, S.

1981 Die Präsensflexion im Bulgarischen. Die Welt der Slaven $X X V I, 2$ (N.F. V, 2), 285-309.

KKEMPGEN, S.

1979 Die Formenbildung des Präsens im Makedonischen. Die Welt der Slaven XXIV, I (N.F. III, 1), 55-65.

1981 'Wortarten' als klassifikatorisches Problem der deskriptiven Grammatik. Historische und systematische Untersuchungen am Beispiel des Russischen. München.

KKEMPGEN, S., LEHFELDT, W.

1978 Das System der Präsensformenbildung im Serbokroatischen. Folia linguistica XII, 1978, 1/2, 137-163.

KKOTELOVA, N.Z.

1974 Искусственный семантический язык (теоретические предпосылки). Вопросы языкознания $1974 / 5,48-63$.

KKRYLOV, N.A.

1963 Типы основ в современном русском языке. Научные доклады Высшей Школы, "Филологические науки" I963/2, 31-44.

FKUBRJAKOVA, E.S.

1965 Что такое словообразование? Москва.

1974 Основы морфологического анализа. Москва.

1978 Об основной единице словообразовательной системы языка. Актуальные проблемы русского словообразования. Ташкент, 36-39.

1980 Семантика производного слова. Аспекты семантических исследований. Москва, 8I-I55.

1981 Типы языковых значений. Семантика производного слова. Москва. 
KÜRSCHNER, W.

1973 Generative Wortbildung 1, 2. Funkkolleq Sprache 1. Frankfurt a.M., 337-350.

KUZNECOV, P.S.

1952 О возникновении и развитии звуковых чередований в русском языке. Известия АН СССР, Отделение литературы и языка II, I952/I, 6I-75.

LEHFELDT, W.

1977 Eine Methode zum synchronen Vergleich der Formenbildung in den slavischen Sprachen. In: Girke,W., Jachnow, $H$. (Hgg.): Slavistische Linquistik 1976. München, 109-128.

1978a K структуре русской парадигмы настоящего времени. Russian Linquistics 4, 1978/1, 41-56.

1978 b Formenbildung des Russischen Verbs. Versuch einer analytisch-synthetisch-funktionellen Beschreibung der Präsens- und der Präteritumflexion. München.

1979 Das System der Präsensformenbildung im Slovakischen. Die Welt der Slaven XXIV, 2 (N.F. III,2), 270-280.

1980 Perspektiven der 'Vergleichenden Grammatik' der slavischen Sprachen. Konstanzer Universitätsreden 94 . Konstanz.

1981 Zur Stellung der Morphonologie innerhalb der Beschreibung des Russischen. Specimina Philologiae Slavicae 30 (Notizen und Materialien zur russistischen Linguistik 6), 45-57.

LEONT'EV, A.A.

1971 Sprache, Sprechen, Sprechtätigkeit. Stuttgart etc.

LEPING, E.I. et al .

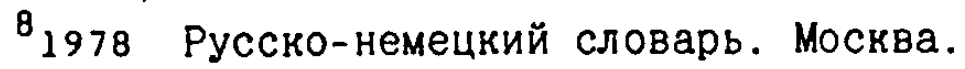

LEVKOVSKAJA, K.A.

1954 Словообразование. Москва

1962 Теория слова, приципы ее построения и аспекть изучения лексического материала. Москва.

LEWANDOWSKI, Th.

1975 Linguistisches Wörterbuch. Bd. 2. Heidelberg.

LICHTMAN, R.I.

1973 К вопросу об основных понятиях словообразовании. Известия АН СССР, Серия литературы и языка $32,1973 / 2$, I32-I4I. 
LLOPATIN, V.V.

1974 Словообразование как объент грамматического описания. Грамматическое описание славянских языков. Москва, $47-60$.

1977 Русская словообразовательная морфемика. Москва.

LLOPATIN, V.V., ULUCHANOV, I.S.

1963 О некоторых принципах анализа слов (к определению понятия слохного слова в современном русском языке). Известия АН СССР, Отделение литературы и языка 22, I 963 , 3. 190-203.

$1969 \mathrm{~K}$ соотношению единиц словообразования и морфонологии. Единицы разных уровней грамматического строя языка и их взаимодеиствие. Москва, І19-1З2.

1972 О формальной (фонематической) близости алломорфов. АКтуальные проблемы русского словообразования I. Самарканд, 201-207.

1978 Теория грамматики и практика грамматического описания. Вопросы языкознания $1978 / \mathrm{I}, 38-50$.

NMAKAEV, E.A, KUBRJAKOVA, E.S.

1969 О статусе морфонологии и единицах ее описания. Единицы разных уровней грамматического строя языка и их взаимодеиствие. Москва, 87-119.

NMAKSIMOV, V.L.

1975 Суффиксальное словообразование имен существительных в русском языке. Ленинград.

N MANUČARJAN, R.S.

1974 К типслогии словообразовательных значений. Известия АН СССР, Серия литературы и языка 33 , 1974/6, 516-526.

1975 Проблемы исследования словообразовательных значений и средств их вырапения. ДД. Ереван.

1978 Значение деривационное и значение словообразовательное. Актуальные проблемы русского словообразования. Тахкент, 12-16.

P MARCHAND, $\mathrm{H}$.

${ }^{2} 1969$ The Categories and Types of Present-Day English Word-

Formation. A Synchronic-Diachronic Approach. München.

1974 Studies in Syntax and Word-formation. Selected Articles by Hans Marchand. On the Occasion of his 65 th Birthday on October lst, 1972. Ed. by Dieter Kastovsky. München.

I MARKOV, V.I., NIKOLAEV, G.A.

1976 Некоторые вопросы теории русского словообразования. Именное словообразование русского языка. Казань, 3-I4. 
MASLOV, JU.S.

1972 Понятие основы и Форматива в дериватологии и в парадигматике. Актуальные проблемы русского словообразования I. Самарканд, 7-Iउ.

MEL'ČUK, I.A.

1963 Several types of linguistic meanings. In: Akhmanova, 0.S. et al.: Exact Methods in Linquistic Research. Berkeley-Los Angeles, 36-43.

1967 К понятию словообразования. Известия АН СССР, Серия литературы и языка $26,1967 / 4,352-362$.

1968 Строение языковых знаков и возможные формально-смысловые отношения мехду ними. Известия АН СССР, Серия литературы и языка $27,1968 / 5,426-438$.

1969 Об определении большей/меньшей смысловой сложности при словообразовательных отношениях. Известия АН СССР, Ceрия литературы и языка 28, I969/2, 126-135.

1972 О супплетивизме. Проблемы структурной лингвистики I97I. Москва, 396-438.

1974 Grammatical meanings in interlinguas for automatic translation and the concept of grammatical meaning. In: Rozencuejg, V.Ju. (ed.): Machine Translation and Applied Linquistics I. Frankfurt a.M., 95-113.

1975 Опыт разработки фрагмента системы понятий и терминов для морфологии (к формализации языка лингвистики). Ceмиотика и информатика 6. Москва, 5-50.

1982 Towards a Language of Linguistics. A System of Formal Notions for Theoretical Morphology. München.

MEL'ČUK, I.A., ŽOLKOUSKIJ, A.K.

1969 К построению действующей модели языка "смысл - текст". Машинный перевод и прикладная лингвистика. Москва, 5-35.

MILOSLAVSKIJ, I.G.

1980 Вопросы словообразовательного синтеза. Москва. MOISEEV, A.I.

1968 Некоторые замечания о книге "Основы построения описательной грамматики современного русского литературного языка". Филологические науки I968/2, IOI-I05.

MOTSCH, W.

1960 Das System der Adjektivableitungsmorpheme in der deutschen Sprache der Gegenwart. Diss. Berlin.

1965 Zur Stellung der Wortbildung in einem formalen Sprachmodell. Studia Grammatica l. Berlin, 31-50. 
MOŽAEVA, I.E. et al. (sost.)

1963 а Славянское языкознание. Библиографический указатель 19I8-I955. Москва.

1963 Славянское языкознание. Библиографический указатель I956-I960. Москва.

NEMCEENKO, V.N.

1971 Словообразование и его место в структуре русского языка. Горький.

NOVICKAJA, V.N.

1968 Словообразование. Состав слова (пособие для студентов заочников филологического факультета). Москва.

OLIVERIUS, Zd.F .

1976 Морфемы русского языка. Praha.

OŽEGOV, S.I.

$11_{1977}$ Словарь русского языка. Москва.

PANOV, M.V.

1970 Об изучении русского словообразования. Известия АН СССР, Серия литературы и языка 29, 1970/3, 258-264.

PLANK, $F$.

1981 Morphologische (Ir-)Regularitäten: Aspekte der Wortstrukturtheorie. Tübingen.

POGREBNAJA, L.L. et al.

21979 Немецко-русский политехнический словарь. Москва.

POLENZ, P. V.

1968 Wortbildung als Wortsoziologie. Wortgeographie und Gesellschaft. Festgabe L.E. Schmitt. Hrsgg. von W. Mitzka. Berlin, 10-27.

1973 Synpleremik I: Wortbildung. Lexikon der germanistischen Linguistik. 8d. 1. Hrsgg. von H.P. Althaus et al. Tübingen, 145-163.

POTICHA, Z.A.

1961 Школьный словообразовательный словарь. Волгоград.

1970 Современное русское словообразование. Пособие для учителя. Москва.

REF ORMATSKIJ, I.G.

${ }^{4} 1967$ Введение в языковедение. Москва.

1975 Фонологические этюды. Москва. 
REVZINA, 0.G.

1969 Структура словообразовательных полей в славянских языках. Москва.

Russkij jazyk

1979 Русский язык. Энциклопедия. Москва.

SANSKIJ, M.N.

1968 Очерки по русскому словообразованию. Москва.

ŠAUMJAN, S.K.

1965 Структурная лингвистика. Москва.

ŠUMJAN, S.K., SOBOLEVA, P.A.

1963 Аппликативная порождающая модель и исчисление трансформаций в русском языке. Москва.

1968 Основания порохдающей грамматики руссного языка. Введение в генотипические структуры. Москва.

1972 словообразовательная и морфемная структура слова. Актуальные проблемы русского словообразования I. Самарканд, $341-350$.

ṠĊERBA, L.V.

1945 Очередные проблемы языноведения. Известия АН СССР, ОТделение литературы и языка 4 , I94575, I73-186.

SËPIR, E

1934 Язык. Москва, Ленинград.

SEREBRENNIKOV, B.A. (red.)

1972 Общее языкознание. Внутренняя структура языка. Москва. SIIRS̉OV, I.A.

1979 Проблемы словообразовательного значения в современной отечественной науке. Вопросы языкознания I979/5, I09-I22.

SMIRNICKI J, A.I.

1954 По поводу конверсии в английском языке. Иностранные языки в школе I954/3, I2-54.

SOBOLEVA, P.A.

1970 Аппликативная грамматика и моделирование словообразования. АДД. Москва.

1972 Моделирование словообразования Проблемы структурной лингвистики 197I. Москва, I65-2I2.

1980 Словообразовательная полисемия и омонимия. Москва. 
STEGMÜLLER, W.

1969 Wissenschaftliche Erklärung und Begründung. Berlin. SUNIK, O.P.

1963 Слово, его основа и корень как различные морфологические категории. Морфологическая структура слова в языках различных типов. Москва, गенинград, 33-52.

ŠVEDOVA, N. Ju .

1966 Основы построения описательной грамматики. Москва.

TICHONOV, A.N.

1967 О семантической соотносительности производящих и производных основ. Вопросы языкознания I967/I, II2-I20.

TIMOFEEV, K.A.

1960 Заметки о словообразовании. Вопросы грамматики. Сборник статей к 75-летию академика И.И. Мещанинова. Москва, Ленинград, 425-433.

1966 О некоторых вопросах словообразования. Новосибирск. TOROPCEV, I. S.

1980 Словопроизводственная модель. Воронем.

ULUCHANOV, I.S.

19700 принципах описания значений словообразовательно мотивированных слов. Известия АН СССР, Серия литературы и языка 29, I970/I, T4-22.

1971 Словообразовательная мотивация и ее виды. Известия АН СССР, Серия литературы и языка 30, I97I/I, 37-46.

1974 Компоненты значения членимых слов. Вопросы языкознания I $974 / 2,7 \mathrm{I}-78$.

1977 Словообразовательная семантика в русском языке. Москва. VINOGRADOV, V.V.

19440 формах слова. Известия АН СССР, Отделение литературы и языка $3,1944 / 1,31-44$.

1952 Словообразование в его отношении к грамматике и лексикологии. Вопросы теории и истории языка в свете трудов И.В. Сталйа. Москва, 99-152.

VINOKUR, G.0.

1946 Заметки по русскому словообразованию. Известия АН СССР, отделение литературы и язнка 5 , I946/4, 315-332.

VOLKONSKY, S., POLTORATZKY, M.

1961 Handbook of Russian Roots. New York. 
VOLOCKAJA, Z.M.

1960 Установление отношений производности между словами. Bопросы языкознания 1960/3, I00-I07.

WE ISGERBER, L.

1964 Vierstufige Wortbildungslehre. Muttersprache 74, 2-12. WORTH, D.S.

1977 A Bibliography of Russian Word-Formation. Columbus, Ohio.

WORIH, D.S., KOZAK, A.S., JOHNSON, D.B.

1970 Russian Derivational Dictionary. New York.

ZALIZNJAK, A.A.

1967 Русское именное словоизменение. Москва.

1977 Грамматический словарь русского язнка. Москва.

ZASORINA, L.N.

1972 Генеративное словообразование и семасиология. Актуальные проблемы русского словообразования I. Самарканд, 63-68.

ZASORINA, L.N. (red.)

1977 Частотный словарь русского языка. Москва.

ZEMSKAJA, E.A.

1966 Понятия производности, оформленности и членимости основ. Развитие словообразования современного русского языка. Москва, 3-12.

1973 Современный русский язык. Словообразование. Москва.

ZEMSKAJA, E.A., KUBRJAKOVA, E.S.

1978 Проблемы словообразования на современном этапе. Вопроси языкознания I978/6, II2-I23.

ZENKOV, G.S.

1969 Вопросы теории словообразования. Фрунзе.

ZVEREV, A.D.

1972 Вырахение направления производности в русском языке. Актуальные проблемы русского словообразования I. СамарКанд, 72-79.

19780 понятии словообразовательной структуре производных. Актуальные проблеми русского словообразования. Ташкент, $74-78$. 


\section{PERSONENREGISTER}

Achmanova, 0.5. $72,215$.

Admoni, V.G. 20, 215.

Altmann, G. $34,215$.

Andreev, N.D. 34,215 .

Apresjan, Ju.D. 76, 215.

Ardentov, B.P. 72,215 .

Armstrong, D. 1,215 .

Arut junova, N.D. $10,215$.

Ašnin, F.D. $1,216$.

Avanesov, R.I. 71, 73, 216.

Avilova, N.S. $1,216$.

Bartosević, A. $37,65,216$.

Baudouin de Courtenay (Boduèn de Kurtenè), I.A. 71, 216.

Bense, M. $13,216$.

Berger, $T$. 2, 216.

Bernistejn, S.B. $71,73,216$.

Bielfeldt, H.H. 97, 216.

Bogorodickij, V.A. 1 .

Budagov, R.A. $66,216$.

Bulygina, T.V. $71,73,216$.

Bykova, L.A. $51,216$.

Carenko, E.I. 36, 217.

Cerepanov, M.V. $10,217$.

Chochlačeva, V.N. SO, 65, 217.

Curganova, V.G. $71,217$.

Debec, N.P. 1, 217.

Dokulil, M. 10,37, 217.

Dressler, W. 14, 217.

Erben, J. 2, 217.

Ermakova, 0.P. $52,217$.

Filin, F.P. $1,218$.

Fleischer, W. $2,56,218$.

Fortunatov, F.F. 1,28 .

Gasparov, B.M. 25, 26, 87, 218.

Gauger, H.M. 2, 218. 
Ginzburg, E.L. 41, 65, 218.

Glejbman, L.V. $10,218$.

Golovin, B.N. $64,218$.

Grucza, F. $3,218$.

Henzen, $W$. 2,218 .

Il'ina, N.E. $71,218$.

Jachnow, $H . \quad 41,219$.

Janceneckaja, M.N. 66, 219.

Janko-Trinickaja, N.A. $34,35,36,64,66,68,219$.

Johnson, D.B. 226.

Kaltwasser, J. $2,219$.

Kempgen, S. $2,20,21,22,23,24,25,26,31,219$.

Kotelova, N.Z. $76,219$.

Kozak, A.S. 226.

Krylov, N.A. $\quad 53,219$.

Kubrjakova, E.S. $7,10,15,37,38,39,43,45,53,54,62$, $63,65,66,69,71,72,73,219,221,: 26$.

Kürschner, $W . \quad 2,220$.

Kuznecov, P.S. $71,220$.

Lehfeldt, w. $\quad v, 2,34,70,73,174,215,220$.

Leont'ev, A.A. 21,220 .

Leping, E.I. $97,220$.

Levkovskaja, K.A. $8,66,220$.

Lewandowski, ih. $71,220$.

Lichtman, R.I. $\quad 51,52,220$.

Lopatin, V.V. $\quad 1,9,33,36,37,47,49,51,54,55,59,61$, $63,67,71,85,86,87,221$.

Makaev, E.A. $71,72,73,221$.

Maksimov, V.L. $63,221$.

Manučarjan, R.S. $60,62,63,64,66,68,75,83,84,86$, 221 .

Marchand, H. 2, 54, 221 .

Markov, V.1. 9, 221.

Maslov, Ju.S. 19, 222.

Mel'ćuk, I.A. $1,12,13,14,18,34,35,41,42,45,46,52$, $53,71,73,76,222$.

Miloslavskij, I.G. $11,28,222$. 
Moiseev, A.I. $9,10,22$.

Motsch, W. 2, 222 .

Możaeva, I.E. 1, 223.

Nemćenko, V.N. 11, 223.

Nikolaev, G.A. 9, 221.

Novickaja, V.N. 1, 223.

01 iverius, Zd.F. $45,71,72,73,223$.

Oíegov, S.I. $\quad 68,75,84,97,223$.

Panov, M.V. $9,223$.

Peśkovskij, A.M. 1 .

Plank, F. 214, 223.

Polenz, P. v. 2, 223.

Poltoratzky, M. 1, 225 .

Poticha, Z.A. 1, 223.

Reformatskij, I.G. 71, 73, 223.

Revzina, 0.G. 60, 63, 224.

Śanskij, M.N. 9, 224.

Sapir, E. (Sèpir, E.) 61, 224.

Ṡaumjan, S.K. 48, 49, 224.

Ścerba, L.V. $10,224$.

Serebrennikov, B.A. $\quad 10,37,38,43,53,54,62,63,224$.

Sidorov, V.N. 71, 73, 216.

Sirsov, 1.A. 65, 224.

Smirnickij, A.I. 8, 9, 224.

Soboleva, P.A. $\quad 1,48,49,65,224$.

Stegmüller, W. 3, 225 .

Sunik, O.P. 19, 225.

Średova, N.Ju. $28,33,36,51,54,55,225$.

Tichonov, A.N. $53,225$.

Iimofeev, K.A. 9,225 .

Toropcev, I.S. 11, 225 .

Uluchanov, I.S. V, 1,9,33,36,37,47,49,51,54,55,59, $60,61,62,63,67,76,85,86,87,221,225$.

Vinogradov, V.V. $1,8,16,19,225$.

Vinokur, G.0. 1, 52, 225.

Volkonsky, S. 1, 225.

Volockaja, Z.M. $40,41,226$. 
We isgerber, L. $2,226$.

Worth, D.S. 1,226 .

Zaliznjak, A.A. 15, 18, 29, 31,61, 77, 97, 226.

Zasorina, L.N. 8, 56, 226.

Zemskaja, E.A. $1,7,9,10,36,55,62,67,226$.

Zenkov, G.S. $45,226$.

Żolkovskij, A.K. 76, 222.

Zverev, A.D. $\quad 52,63,226$. 


\section{SACHREGISTER'}

Adjektiv $3, \underline{24}$

- deadjektivisches A. (in allgemeiner Explikation) 85f

- denumerales A. "

- desubstantivisches A. " $77 \mathrm{f}$

- deverbales A. " $79 f$

- nichtabgeleitetes A. " 77

- zusammengesetztes A. " $81 \mathrm{ff}$

Adverb 26

- deadjektivisches A. (in allgemeiner Explikation) 87

Affix $5, \underline{36}$

Ähnlichkeit

- formale $\ddot{A}$. $18, \underline{45 \mathrm{ff}}$

- inhaltliche Ä. $46 \mathrm{ff}$

Alternation

- morphonologische A. $69, \underline{71 f}$

- neophonetische A. 71

- paleophonetische A. 71

analytisch-synthetisch-funktionelle Methode 2 , 3ff applikatives generatives Modell $10, \underline{48 f}$ Basisform 70

Bedeutung

- allgemeine kategoriale B. 16, 20f

- grammatische $8.2, \underline{17}$

- lexikalische B. 20,33

- nichtgrammatische B. $2, \underline{17}$

Belastung

- funktionelle B. $4, \underline{197 \mathrm{ff}}$

Derivatemtyp 200

Derivationsbeziehung $5, \underline{43 \mathrm{f}}$

Derivationsrichtung $5, \underline{56}$

- Bestimmung der D.

1 Das Register enthält lediglich das zum Verständnis der Arbeit Wichtigste. Dabei ist zunächst die Stelle des ersten Auftretens genannt, sodann (unterstrichen) die mit dem für den Leser größten Informationswert. Sind beide Stellen identisch, wird nur diese eine angeführt. 
- formales kriterium $49 f f$

- inhaltliches Kriterium $49 \mathrm{ff}$

- quantitatives Kriterium $55 f f$

Derivator 66

Diachronie 8

Diskretheit $\quad 30$

Distribution

- des Signifikanten 14

- des Signifikats 14

Exklusionsrelation $42 \mathrm{f}$

Explikation

- verallgemeinerte E. nichtgrammatischer Inhaltselemente $S$, $\underline{75 f}$

Flexionsendung $27, \underline{29}$

Form

- primäre F. $38 \mathrm{f}$

- sekundäre $F$. $38 \mathrm{f}$

Formant $37, \underline{67 f}$

Grammem $16, \underline{18}$

Graph $\quad 175 \mathrm{ff}$

Häufigkeit

- pragmatische H. 55,57

- systemische H. 55

Identifikationsoperation $15 \mathrm{f}$

Identitätsrelation $\quad 42 \mathrm{f}$

Inklusionsrelation $42 \mathrm{ff}$

Intersektionsrelation $42 \mathrm{ff}$

Kategorie

- grammatische K. 16, 18

Kombinationen

- maximale K. $97 \mathrm{ff}$

- minimale K. $97 \mathrm{ff}$

- reale K. $97 \mathrm{ff}$

Kontext

- des Signifikanten 14

- des Signifikats 14

Lexem 2,18 


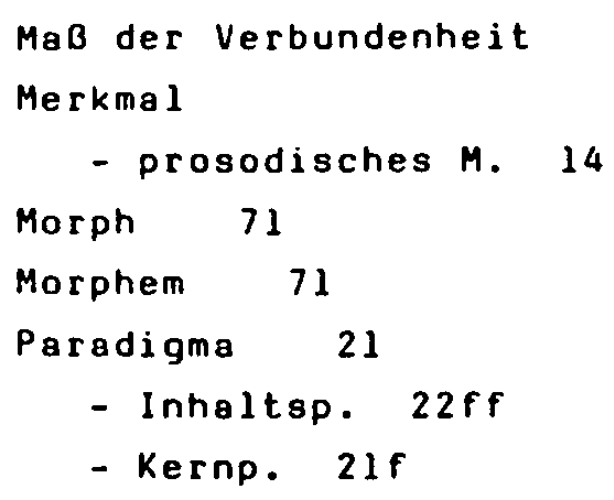

\section{Phonem 14}

Rede 13

Redeteile 2, $19 \mathrm{ff}$

Regelhaftigkeit

- implikative R. 175

- prädiktive R. $176 \mathrm{f}$

Relator $48 \mathrm{f}$

Semion $\quad 48 \mathrm{f}$

Signifikant $\quad 13$

Signifikat 13

Sprache

- natürliche S. 13

Stamm

- motivierender 70

- Wortformst. 27, $31 f$

\section{Stärke}

- diskriminatorische S. 6, $197 \mathrm{ff}$

- identifikatorische S. 6

- prädiktive S. $197 \mathrm{ff}$

Stichprobe

- pragmatische S. 57

Substantiv $17, \underline{24}$

- deadjektivisches S. (in allgemeiner Explikation) $83 \mathrm{ff}$

Suppletivismus $45, \underline{48}$

Symmetrie $29 \mathrm{ff}$

Synchronie $8 \mathrm{f}$

rext

- konkrter T. in der natürlichen Sprache 13

Transposition $\quad 38$ 


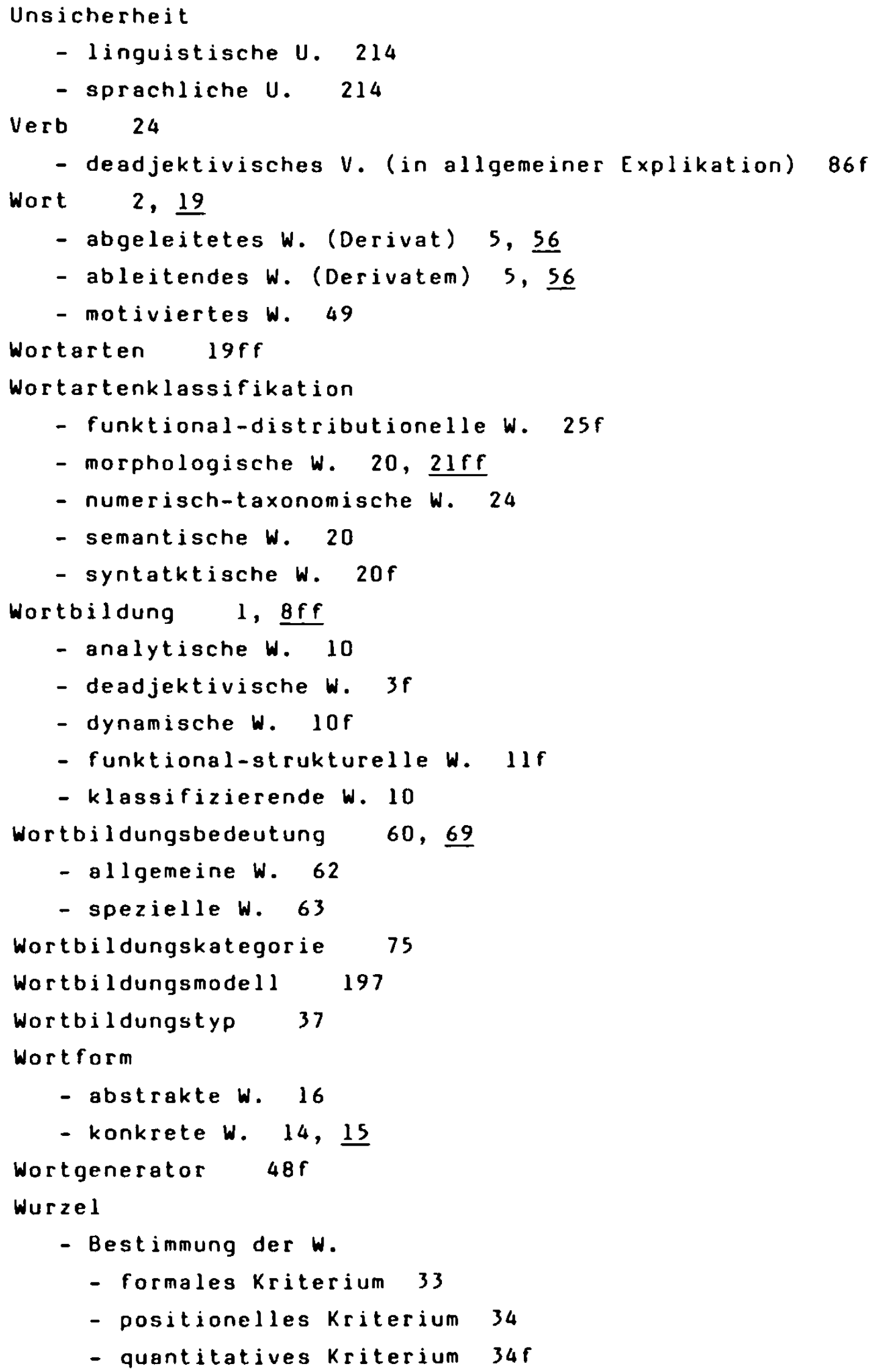


$-235$

- semantisches Kriterium 33

- Wortformw. 32, 35

Zeichen

13 
$\therefore$

-

. $131+5$

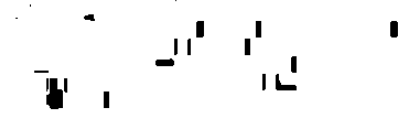

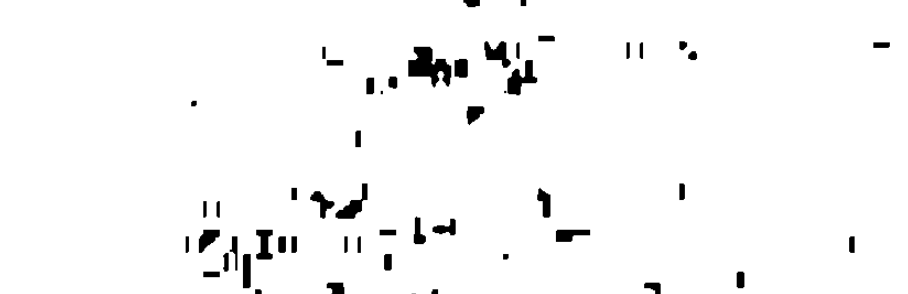

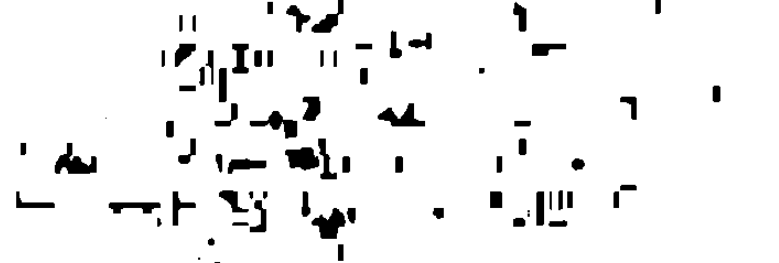

I 
162. Neurelter, F.: Weibrussische Anthologie. Ein Lesebuch zur weibrussischen Iiteratur (mit deutschen Ubersetzungen). 1983. $230 \mathrm{~S}$.

163. Witte, G.: Die sowjetische Kolchos- und Dorfprosa der funfziger Jahre. Zur Evolution einer literarischen Unterrethe. 1983. X, $292 \mathrm{~S}$.

164. Timroth, W.v.: Russische und sowjetische Soziolinguistik und tabulsierte Varletäten des Russischen. 1983. VIII, $194 \mathrm{~S}$.

165. Christians, D.: Die Sprachrubrik in der Literaturnaja gazeta von 1964 bis 1978. Dokumentation und Auswertung. 1983. $266 \mathrm{~S}$.

166. Roschmal, W.: Das poetische System der Dramen I.S. Turgenevs. Studien zu einer pragmat Ischen Dramenanalyse. 1983. X, $453 \mathrm{~S}$.

167. Hofmann, T.: Das Bauerntum in der sowjetrussischen Prosa der 20er Jahre. Konzeptionen, Konflikte und Figuren. 1983. $434 \mathrm{~S}$.

168. Morsbach, P.: Isaak Babel' auf der sowjetischen Büne. 1983. $\mathrm{X}, 255 \mathrm{~S}$.

169. Tutschke, G.: Die glagol1t1sche Druckere1 von Rijeka und ihr histor lographisches Werk Rnizice od îtié rimskih arhieréov 1 cesarov. 1983. $373 \mathrm{~S}$.

170. Lam, A.: Malnzer Vorlesungen Uber die polnische Literatur se1t 1918. 1983. IV, $280 \mathrm{~S}$.

171. Pratt, S.: The Semantics of chaos in Tjutčev. 1983. VIII, $149 \mathrm{~S}$.

172. Slavistische Iinguistik 1982. Referate des VIII. Ronstanzer Slavistischen Arbe1tstreffens Kiel 28.9. - 1.10.1982. Herausgegeben von Hans Robert Mehlig. 1983. $262 \mathrm{~S}$.

173. Dingley, J.: The Peripheral Plural Endings of Nouns in Petrine Sermons. 1983. VIII, 388 S.

174. Hoelscher-Obermaler, H.-P.: Das lyrische Werk Antonl Langes. Untersuchungen zur Dichtungssprache eines, jungpolnischen" Autors. 1983. $127 \mathrm{~S}$.

175. Bojie, V., W. Oschlies: Lehrbuch der mazedonischen Sporache. 1984. $185 \mathrm{~S}$.

176. Roedel-Rappl, C.: Analogle und Sprachwandel im Vergleich zweier verwandter Sprachen: Russisch und Polnisch. 1984. $\mathrm{X}, 246 \mathrm{~S}$.

177. Kattein, R.: Die Pronominalsysteme der slavischen Sprachen. 1984. IV, $142 \mathrm{~S}$.

178. Wust, H.: Tradition und Innovation in der sowjetrussischen Dorfprosa der sechziger und slebziger Jahre. zu Funktion, Darstellung und Gehalt des dörflichen Helden bel Vasilij Sukšin und Valentin Rasputin. 1984. VIII, $249 \mathrm{~s}$.

179. Vogl, J.: Das Frühwerk Valentin P. Kataevs. 1984. VIII, $197 \mathrm{~S}$.

180. Aspekte der Slavistik. Festschrift fur Josef Schrenk. Herausgegeben von Wolfgang Girke und Helmut Jachnow. ig84. $270 \mathrm{~S}$. 
181. Slavistische Linguistik 1983. Referate des IX. Konstanzer Slavistischen Arbeitstreffers München 27. mit 29.9.1983. Herausgegeben von Peter Rehder. 1984. $282 \mathrm{~S}$.

182. Penzkofer, G.: Der Bedeutungsaufbau in den späten Erzärlingen Cechovs. "Offenes" und "geschlossenes" Erzählen. 1984. $367 \mathrm{~s}$.

183. Kammer, G.: Probleme bel der Ubersetzung von phraseologischen Einheiten aus dem Russischen ins Deutsche (anhand von Werken V.F. Panovas). 1985. VIII, 223, XXV S.

184. Slavistische Linguistik 1984. ReEerate des X. Konstanzer Slavistischen Arbeitstzeffens Konstanz 11. mit 14.9.1984. Herausgegeben von Werner Lehfeldt. 1985. $359 \mathrm{~S}$.

185. Kahlenborn, U.: Goethes Lyrik in russischer Ubersetzung. V.A. Zukovskij und F.I. TjutCev als bedeutendste Goethe-ubersetzer der russischen Romantik. 1985. XIV, $309 \mathrm{~S}$.

186. Hauenschild, $\mathrm{Ch}$.: Zur Interpretation russischer Nominalgruppen. Anaphorische Bezüge und thematische Strukturen im Satz und im Text. 1985. X, $391 \mathrm{~S}$.

187. Rathmayr, R.: Die russischen Partikeln als Pragmalexeme. 1985. $354 \mathrm{~S}$.

188. Boss, D.: Das sowjetrussische Autorenlied. Eine Untersuchung am Beispiel des Schaffens von Aleksandr Galic, Bulat Okudžava und Vladimir Vysockij. 1985. VIII, $119 \mathrm{~S}$.

189. Hiller, P.: D.I. Fonvizin und P.A. Plavil'šcikov. Ein Kapitel aus der russischen Theatergeschichte im 18. Jahrhundert. 1985. X, $344 \mathrm{~S}$.

190. Kaltwasser, J.: Die deadjektivische Wortbildung des Russischen. Versuch einer, analytisch-synthetisch-funktionelien' Beschreibung. 1986. VIII, 235 S.

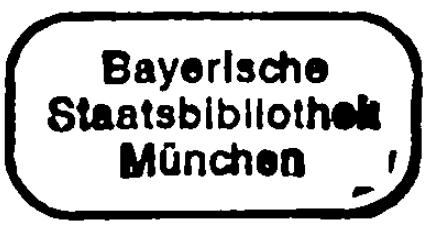

